\title{
Hildegard Spraul
}

\section{Untersuchungen \\ zur Satzsemantik russischer \\ Sätze mit freien Adverbialen}

\section{Am Beispiel von Lokal-, Temporal- und Modaladverbialen}

Verlag Otto Sagner München - Berlin - Washington D.C.

Digitalisiert im Rahmen der Kooperation mit dem DFG-Projekt „Digi20“

der Bayerischen Staatsbibliothek, München. OCR-Bearbeitung und Erstellung des eBooks durch den Verlag Otto Sagner:

http://verlag.kubon-sagner.de

( $)$ bei Verlag Otto Sagner. Eine Verwertung oder Weitergabe der Texte und Abbildungen, insbesondere durch Vervielfältigung, ist ohne vorherige schriftliche Genehmigung des Verlages unzulässig. 
SPECIMINA PHILOLOGIAE SLAVICAE

Herausgegeben von

Olexa Horbatsch, Gerd Freidhof und Peter Kosta

Supplementband 21

Hildegard Spraul

\title{
UNTERSUCHUNGEN \\ ZUR SATZSEMANTIK RUSSISCHER SÄTZE MIT FREIEN ADVERBIALEN
}

\author{
Am Beispiel von \\ Lokal-, Temporal- unet Modaladverbialen
}

VERLAG OTTO SAGNER - MUNCHEN

1986 


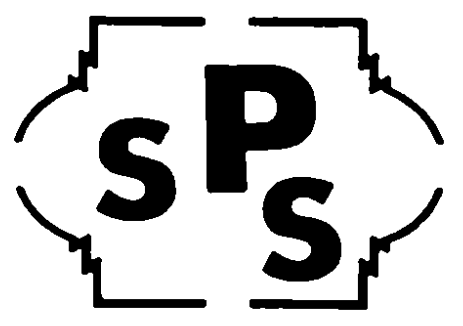

ISBN $3-87690-361-0$

Copyright by Verlag otto Sagner, München 1986. Abtellung der Firma Kubon und Sagner, München. Druck: Görich \& Weiershãuser, 3550 Marburg/L.

$$
\text { P871.6195 }
$$


Meinen Lehrerinnen der russischen Sprache in dankbarer Erinnerung

Marija Georgievna Wlassova-Terechova (1900 - 1967)

Ekaterina Fedorovna Makarova (1898 - 1978) 
6 of

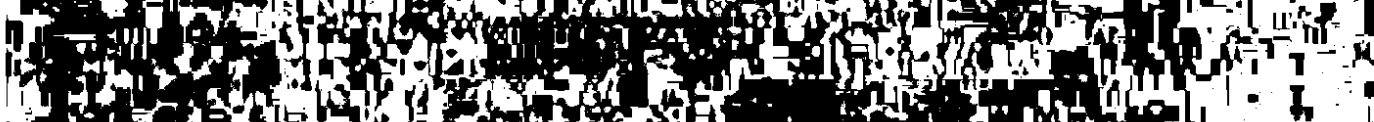

(1)

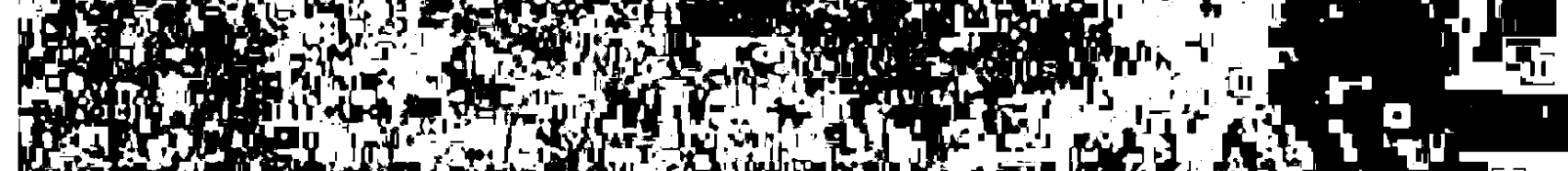

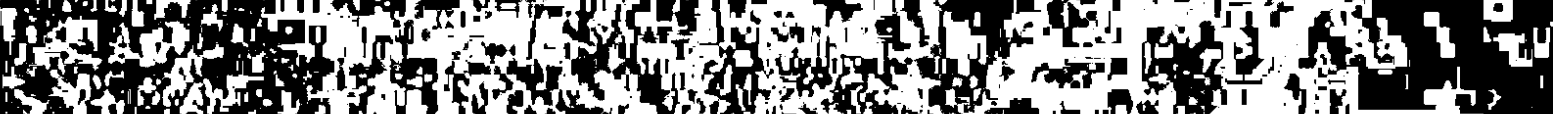

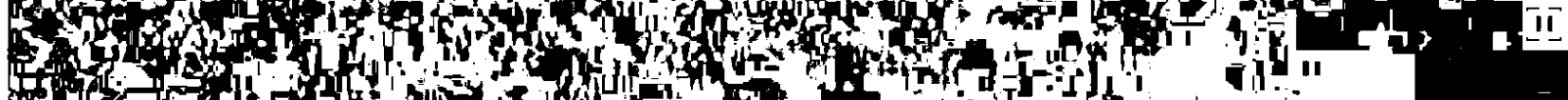

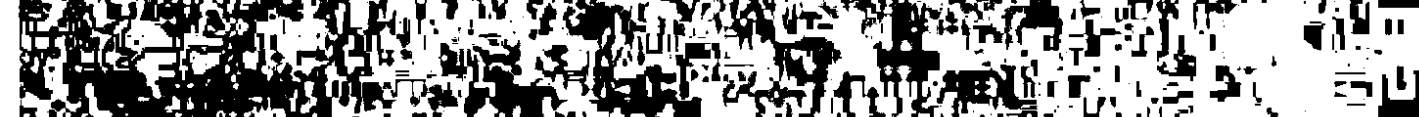

For

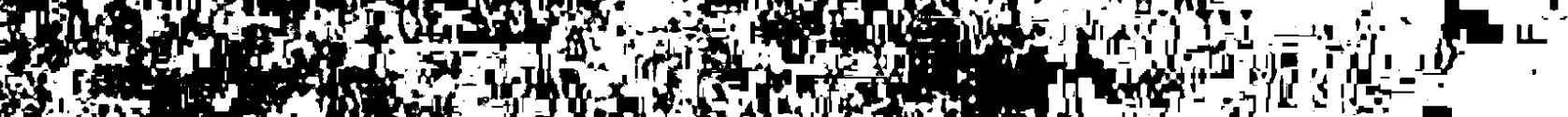

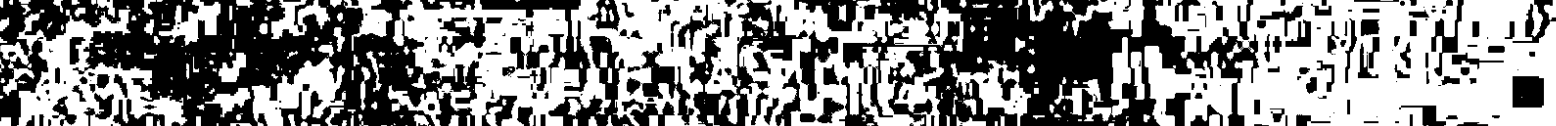

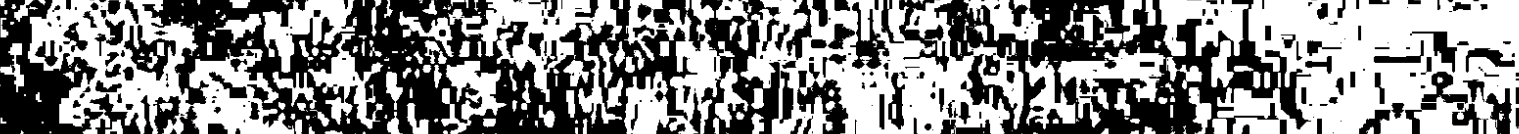

-

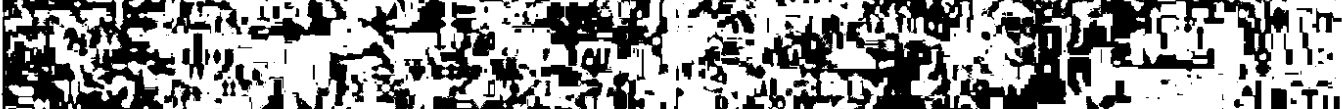

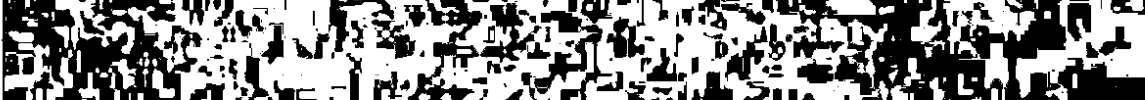

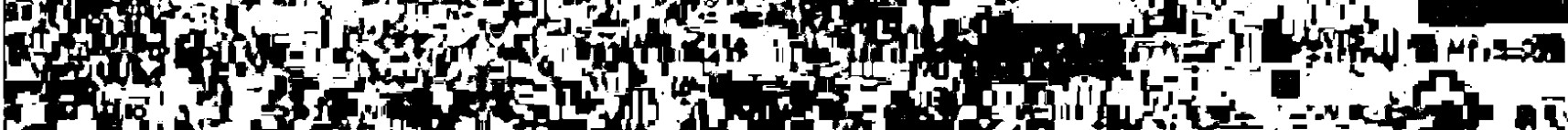

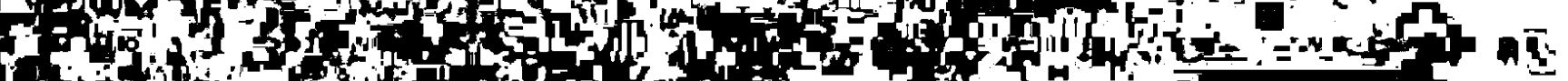
fory

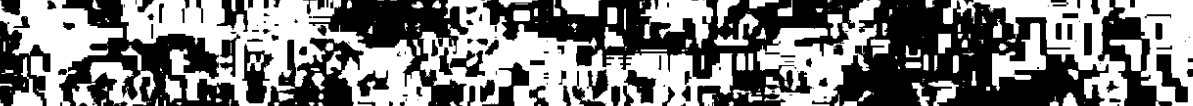

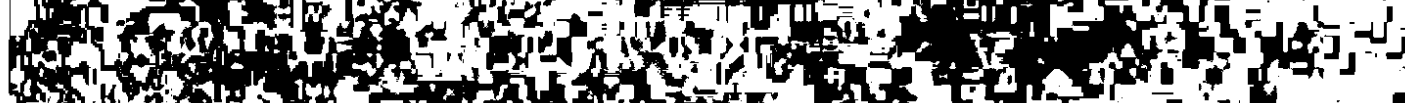

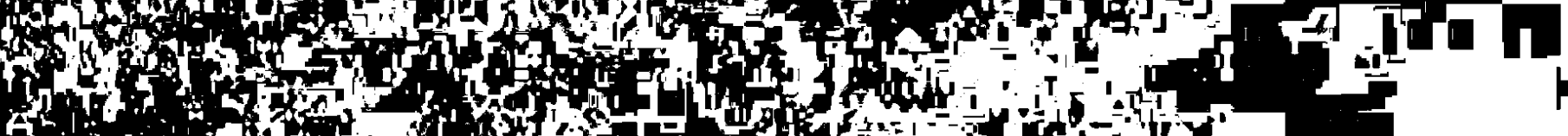

$+$

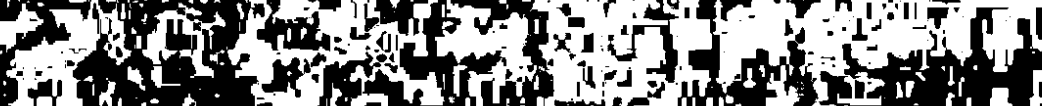

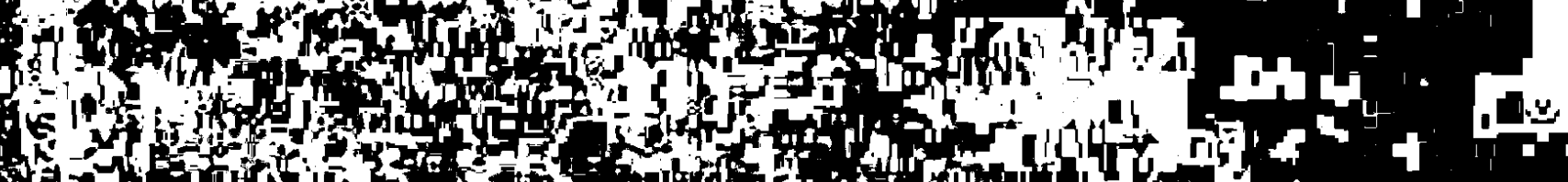

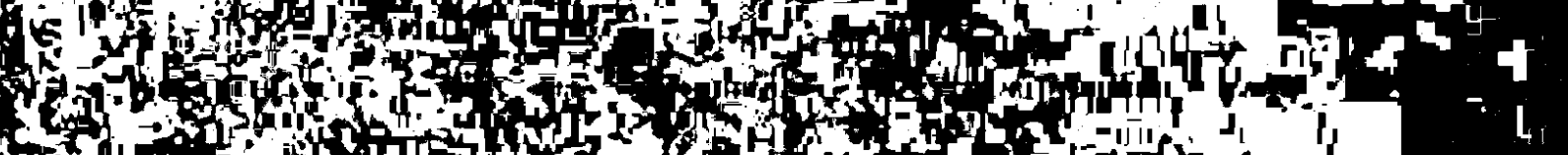

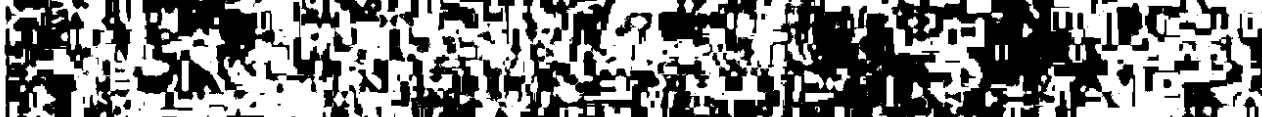

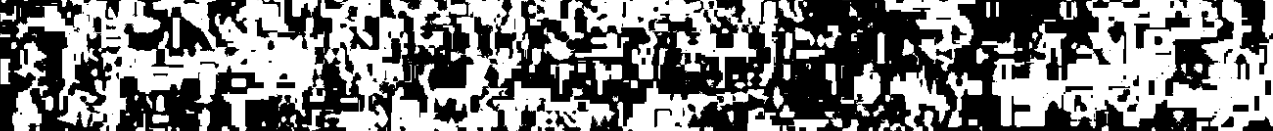

-

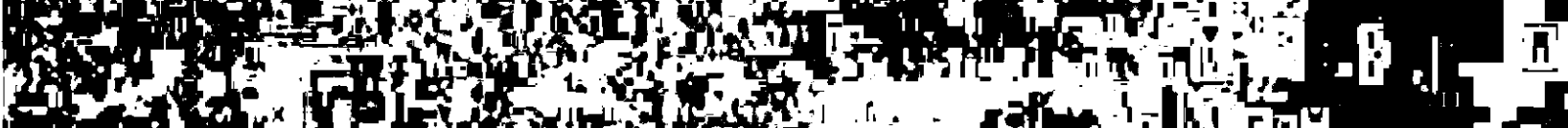

An

P-

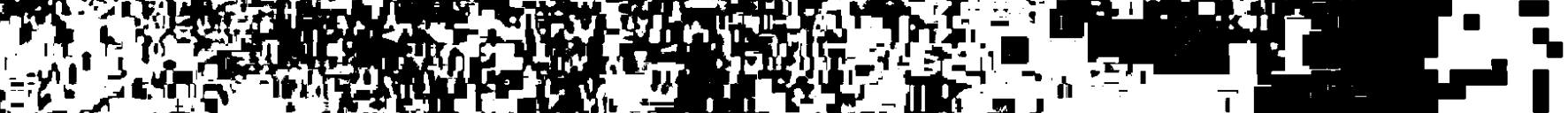

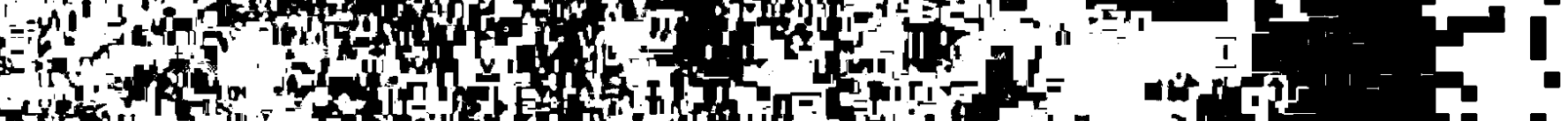
40

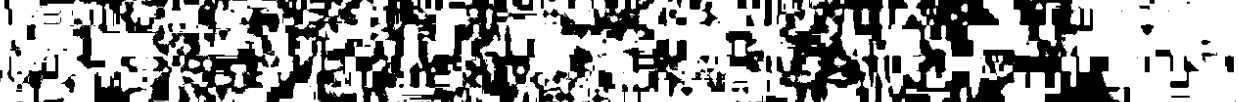

if

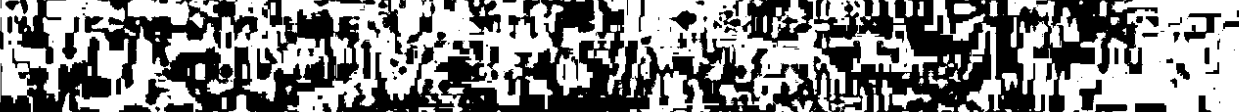

(s) of

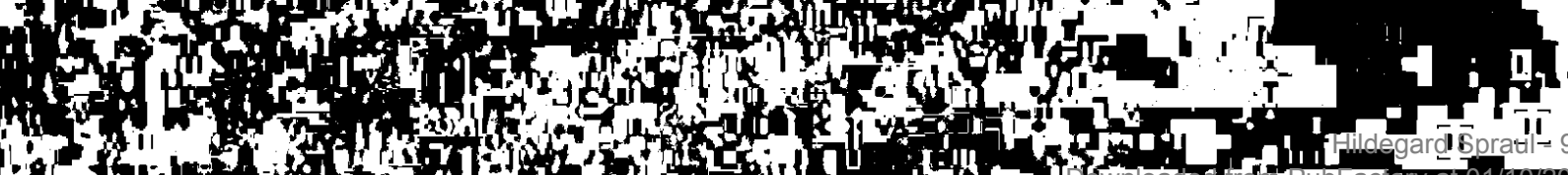


VORWORT

Adverbiale stellen in Syntaxbeschreibungen auch recht unterschiedlicher theoretischer Positionen ein oft und nicht selten unbefriedigend behandeltes Thema dar.

Beschreibungen der Adverbiale, die in der Tradition von Chomsky's "Aspects of the Theory of Syntax" (1965) stehen, gelingt es zwar, die Adverbialphrasen zu klassifizieren und den obligatorischen und fakultativen Adverbialphrasen eine unterschiedliche syntaktische Beschreibung im Satzrahmen zu geben (z.B. R. Steinitz, 1971). Während jedoch die obligatorischen Adverbiale als zum Verbnucleus gehörend und durch Selektionsregeln differenziert beschrieben werden können, entziehen sich die fakultativen Adverbiale in dem so konzipierten Syntaxmodell einer expliziten Einordnung.

In stärker traditionell ausgerichteten Grammatiken, die die Lehre vom Satz als eine oberflächenorientierte Beschreibung der Satzglieder verstehen, finden sich zu der syntaktischen Einordnung der Adverbiale oft nur vage Bemerkungen. So heiBt es in den sowjetischen Akademiegrammatiken der modernen russischen Schriftsprache (Moskva 1954 ff; Moskva 1970; Moskva 1982) zu den Adverbialen - gemeint sind die fakultativen - fast stereotyp: otnosjastiesja "ko vsemu predloženiju v celom" (Grammatika (1970), 624).

Die vorliegende Arbeit unternimmt den Versuch, den syntaktischen Stellenwert der fakultativen Adverbiale im Satz zu ermitteln und ihre Beziehungen $z u$ den übrigen Gliedern $z u$ bestimmen. Sie konzentriert sich auf die fakultativen Adverbiale in Form von Präpositionalphrasen (PP). Daß die fakultativen Adverbiale Einschränkungen ihrer Beziehungen im Satz unterworfen sind, zeigen schon Sätze wie: (1) "Die Kinder sahen eine Katze im Garten."/"Die Kinder sahen im 
Garten eine Katze"/"Die Kinder im Garten sahen eine Katze."/ (2) "Die Kinder sahen eine Katze am Abend."/"Die Kinder sahen am Abend eine Katze."/*Die Kinder am Abend sahen eine Katze."/

Für das Russische gilt teilweise Entsprechendes - $\mathbf{s}$ Beispielsets (1) und (3) - andererseits werden Unterschiede deutlich - s. Beispielsets (2) und (4): (3) "Deti videli koక̌ku v sadu."/"Deti videli v sadu koł̌ku."/"Deti v sadu videli košku."/(4) "Deti videli košku vełerom."/"Deti videli vełerom košku"/"Deti vełerom videli košku."/

Die Beispiele machen deutlich, daß die verwendung der fakultativen Adverbiale wohl von so unterschiedlichen Faktoren bedingt ist wie: Bezugsmöglichkeiten $z u$ anderen Satzgliedern, Stellung im Satz, Klassenzugehörigkeit innerhalb der Adverbiale.

Für die Behandlung der sich damit stellenden linguistischen Probleme wurde für die vorliegende Arbeit ein Beschreibungsansatz gewählt, der Hypothesen über die Beziehungen zwischen syntaktischen Formen und ihren Funktionen formuliert und diese in der Tradition generativer Transformationsgrammatiken expliziert. Im besonderen lehnt sich diese Arbeit an das Prager Konzept der "Functional Generative Grammar" (1973) und deren neueren Entwicklungen an. Es wird ein Satzmodell entwickelt, in dem die fakultativen Adverbiale auf prädikative Ausdrücke zurückgeführt werden. Die Uberprüfung des Modells an den sprachlichen Gegebenheiten erfolgt mit Hilfe von Distributionstests, wobei im Mittelpunkt Prädikationsund Dialog-Tests stehen. Die Prädikations-Tests im besonderen decken syntaktische Homonymien und synonymien auf und beschreiben diese; mit den Dialog-Tests wird die Rolle der fakultativen Adverbiale für die aktuelle Gliederung von Aussagen untersucht; sie weisen damit auch über die Satzgrenze hinaus. 
Die Subklassifizierung der Adverbiale erfolgt in Anlehnung an prädikatenlogische Untersuchungen (Bartsch, 1972). Innerhalb dieses Beschreibungssystems werden die verschiedenen Subklassen der Adverbiale als unterschiedliche Prädikationen (lokal, temporal) über bestimnte Klassen von Argumenten dargestellt. Dabei werden die semantischen Relationen zwischen Prädikation und Argument als subkategorisierende Eigenschaften genereller Natur verstanden und von spezifisch lexikalisch bedingten Selektionsmerkmalen einzelner Lexikoneinträge abgegrenzt.

Die Untersuchung der fakultativen Adverbiale in dem skizzierten Beschreibungsansatz zeigt die Möglichkeiten, aber auch die Grenzen, die mit dem Versuch verbunden sind, eine formal schwach determinierte Klasse sprachlicher Ausdrücke - und als eine solche betrachte ich die fakultativen Adverbiale innerhalb einer grammatischen Theorie $\mathrm{zu}$ beschreiben, die sich expliziter Untersuchungsmethoden bedient. Ich habe deshalb die Untersuchung auf die 3 Klassen: lokale, temporale und modale Adverbiale beschränkt, die aufgrund ihrer sprachlichen Gegebenheiten als elementare Klassen fakultativer Adverbiale bezeichnet werden können.

Die Schwierigkeit der in der Arbeit beschriebenen Thematik läßt erahnen, daß unterschiedliche Beschreibungsansätze und Interpretationen im Detail vorgenommen werden können. Solche sind in der wissenschaftlichen Diskussion und Auseinandersetzung nicht nur erwünscht, sondern sogar notwendig. DaB auch die Herausgeber der Reihe, denen ich für die Aufnahme der Arbeit sehr herzlich danke, in einigen Punkten andere Positionen vertreten, scheint daher fast selbstverständlich zu sein.

Die vorliegende Untersuchung ist die überarbeitete Fassung meiner im Jahre 1983 von der Philosophischen Fakultät der 
Georg-August-Universität Göttingen angenommenen Habilitationsschrift.

Für ihre vielseitige Unterstützung, wertvollen Hinweise und anregenden Gespräche danke ich Herrn Professor A. de Vincenz und Frau Professor I. Mahnken.

Besonderen Dank habe ich Herrn Professor P. Sgall für seine kritischen und hilfreichen Kommentare abzustatten, sowie Frau Dr. J. Panevová für ihren fachlichen Rat.

Zu weiterführenden Diskussionen stellten sich dankenswerterweise die Professoren A. Bogusławski, Th. Gardner, R. Rưziðka und W. Schmid zur verfügung.

Nicht zuletzt gilt meine dankbare Erinnerung meinem Lehrer Professor Dmitrij Tschižewskij.

Zu danken habe ich auch Frau V. Meskemper, die das Manuskript mit großem persönlichen Einsatz, gewissenhaft und umsichtig erstellte.

Saarbrücken, im November 1985

Hildegard Spraul 
0. Symbole und Abkürzungen

1. Einleitung

2. Zur Methode

2.1 Adverbiale in neueren Sprachbeschreibungsmodellen

2.1.1 Adverbiale in einem Modell mit Transformationen nach Harris

2.1.2 Adverbiale in einem Modell nach Chomsky's

"Aspekte der Syntaxtheorie"

2.1.3 Adverbiale in der "Funktional Generativen Beschreibung" (Prag)

2.1.4 Adverbiale in der "Adverbialsemantik" von

R. Bartsch

2.1.5 Die "Tiefenstruktur" in neueren Arbeiten

R. Růžičkas und seines Kreises

2.2 Theoretische und methodische Schlußfolgerungen

3. Lokale Adverbiale (PP loc')

3.1 Das Lokaladverbial in der Funktion einer Prädikation

3.1.1 Die Beziehungen zwischen syntaktischer Ebene (VP PP ${ }_{10 c}$ ) und semantischer Ebene ( $S^{1}, s^{2}$ )

3.1.2 Der Satzkonnektor von $\mathrm{S}^{1}$ und $\mathrm{S}^{2}$ im Zusammenhang mit der $\mathrm{TF} \mathrm{S}^{2} \Longrightarrow \mathrm{PP}_{10 \mathrm{C}}$

3.1.3 Das Verhältnis der Tf $\mathrm{S}^{2} \mathrm{TS}_{\mathrm{TS}} \Longrightarrow{ }_{10 \mathrm{C}}^{\mathrm{zU} \text { anderen }}$ Tf des Satzes

3.2 Prädikation durch PP ${ }_{\text {loc }}$ und aktuelle Satzperspektive

3.2.1 Sätze mit PP in Endstellung

3.2.2 Sätze mit PP loc vor und nach dem finiten verb 106

3.2.2.1 Sätze mit PP ${ }_{\text {loc }}^{\text {loc }}$ vor dem finiten verb 106

3.2.2.2 Sätze mit PP ${ }_{10 c}$ nach dem finiten Verb 114 
3.2.3 Sätze mit $\mathrm{PP}_{\text {loc }}$ in Anfangsstellung

4. Temporale Adverbiale (PP temp)

4.1 Das Temporaladverbial in der Funktion einer Prädikation

4.1.1 Die Ableitung der TS von $\mathrm{S}_{\mathrm{OF}} \rightarrow \mathrm{NP} \operatorname{VP}(\mathrm{X})$ PP (1)

4.1.2 Die Ableitung der TS von $\mathrm{S}_{\mathrm{OF}} \rightarrow \mathrm{NP} \operatorname{VP}(\mathrm{X})$ $\mathrm{PP}$ temp

4.2 Zum Problem der Hierarchie der Prädikationen in Sätzen mit Temporaladverbial

4.3 Temporaladverbial und Verbalaspekt

4.4 Zum Problem der Mehrdeutigkeit von Sätzen mit Temporaladverbial

4.4.1 Mehrdeutige Relationsmöglichkeiten der PP 166

4.4.2 Mehrdeutigkeit der Klasse der Adverbialen temp

5. Modale Adverbiale ( $P P$ mod)

5.1 Der Negations-Test be $i$ Modaladverbialen

5.2 Modale Adverbiale vom Typ (VP v NP ${ }_{\text {präp }}$ ): Mod ${ }_{1}$

5.3 Prädikation des Satzsubjekts/-objekts vom Typ

5.4 Modale Adverbiale vom Typ (VP s NP ${ }_{\text {instr }}$ ): Mod ${ }_{2} 208$

5.5 Modale Adverbiale vom TYp (VP po NP dat $^{\text {inst }}: \operatorname{Mod}_{3}{ }^{2}$

6. Exkurse

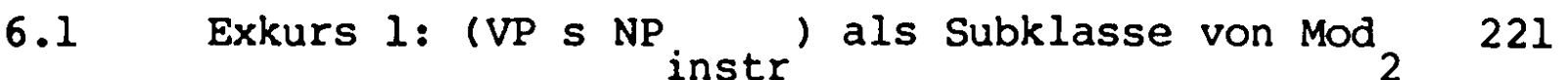

6.2 Exkurs 2: PP (bez NP gen )

7. Zusammenfassung

8. Anhang

8.1 Anmerkungen

8.2 Literaturverzeichnis

Englische und russische Zusammenfassung 
0. SYMBOLE UND ABKURZUNGEN

Symbole

\begin{tabular}{|c|c|}
\hline A & - Adjektiv \\
\hline $\begin{array}{l}\text { A präd } \\
\text { Adv }\end{array}$ & $\begin{array}{l}\text { - A als Prädikatsnomen } \\
\text { - Adverbial (obligatorisch) }\end{array}$ \\
\hline Advb & - Adverbial (frei) \\
\hline AdvbTemp & - Temporaladverbial (lokalisierend) \\
\hline AdvbTemp quant & - Temporaladverbial (quantifizierend) \\
\hline akt & - aktiv (Verbalgenus) \\
\hline cop & - Kopula \\
\hline HS & - Hauptsatz \\
\hline $10 \mathrm{c}$ & - lokal \\
\hline $\bmod$ & - modal \\
\hline $\mathrm{N}$ & - Nomen \\
\hline NP & - Nominalphrase \\
\hline NP & - NP im Nominativ \\
\hline $\begin{array}{l}\mathrm{NP}_{8}^{\mathrm{n}} \text { etc. } \\
\mathrm{NP}^{1 / 2} \\
\mathrm{NP}^{1 / 2 \mathrm{~S}^{1}}\end{array}$ & $\begin{array}{l}\text { - NP im Genetiv etc. } \\
\text { - erste, zweite ... n-1 NP im Satz } \\
-{ }^{1}{ }^{2}, \text { dominiert von } s^{1}\end{array}$ \\
\hline $\mathrm{NP}_{\text {präd }}$ & - NP als Prädikatsnomen \\
\hline NPX & - NP mit noch festzulegender Relation \\
\hline $\begin{array}{l}\text { NP } \\
\text { Nerb } \\
\text { NS zeit }\end{array}$ & $\begin{array}{l}\text { - nominalisiertes Verb } \\
\text { - nominaler Ausdruck des Zeitsystems } \\
\text { - Nebensatz }\end{array}$ \\
\hline OF & - Oberflächenstruktur \\
\hline Part & - Partizip \\
\hline pass & - passiv (Verbalgenus) \\
\hline$P P+S$ & - Präpositionalphrase \\
\hline $\begin{array}{l}\text { PP TS } \\
\text { Präd }^{-S} \text { TS }\end{array}$ & $\begin{array}{l}\text { - PP, abgeleitet von } S_{T S} \\
\text { - PP, nicht abgeleitet von } S_{T S}\end{array}$ \\
\hline Präp & - Präposition \\
\hline Prof & - Proform \\
\hline Prop & - Proposition \\
\hline
\end{tabular}




$$
\begin{aligned}
& \begin{array}{r}
Q_{1}, 2 \ldots n-1 \quad \text { - erste, zweite } . . . \text { Frage nach } \\
\text { Thema-Rhema-Gliederungs-Test }
\end{array} \\
& \text { rel - relativ } \\
& \text { S - Satz } \\
& \text { \#S\# - Satz als Ausgangssymbol der TS } \\
& S \quad-S \text { kontextfrei } \\
& S \text { KS - S kontextsensitiv } \\
& S \\
& \text { konst } \\
& \mathrm{s} \\
& \text { matr } \\
& S \\
& \mathrm{~T}-\mathrm{C}-\mathrm{A} \\
& \text { - Konstituentensatz } \\
& \text { - Matrixsatz } \\
& \text { - S der Tiefenstruktur } \\
& \text { - }\left\{\begin{array}{l}
\text { Topic-Comment-Articulation } \\
\text { Thema-Rhema-Gliederung }
\end{array}\right\} \\
& \text { temp - temporal } \\
& \text { TF - Transformation } \\
& \stackrel{\mathrm{TF}}{\Longrightarrow} \quad \text { - Negations-TF } \\
& \text { Neg }
\end{aligned}
$$

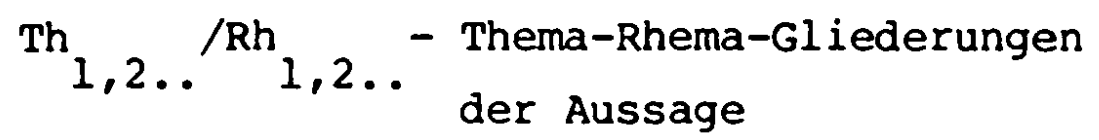

$$
\begin{aligned}
& \text { TS - Tiefenstruktur } \\
& \text { ups - unpersönliche Form des Verbs } \\
& \mathrm{V} \text { - Verb } \\
& \text { Vb - Verb als Prädikat im engeren } \\
& \text { sinn } \\
& \mathrm{Vb}-\quad-\mathrm{Vb} \text { im unvollendeten Aspekt } \\
& \mathrm{Vb}_{\mathrm{VA}}^{\mathrm{UA}} \quad-\mathrm{Vb} \text { im vollendeten Aspekt } \\
& V^{V A} \quad-\text { Verbalphrase } \\
& \text { (X) - fakultatives Glied im Satz } \\
& \phi_{C} \quad \text { - sentence connective operator } \\
& \theta \quad-\text { Nullstelle } \\
& \text { ( ) - fakultativ besetzbar } \\
& \{\} \text { - alternativ } z u \text { verwenden } \\
& \longrightarrow \quad \text { - wird expandiert } z \mathrm{u} \\
& \Longrightarrow \quad \text { - wird transformiert } \mathrm{zu} \\
& \longleftrightarrow \quad \text { - entspricht } \\
& +\quad-\text { Testergebnis positiv } \\
& \text { - Testergebnis negativ }
\end{aligned}
$$




\begin{tabular}{ll}
\hline ASG Bericht & - Arbeitsstelle Strukturelle Grammatik \\
& - Bericht \\
FGB & Funktional Generative \\
& Beschreibung \\
Grammatika & - Grammatika sovremennogo russkogo \\
(1970) & literaturnogo jazyka, Moskva, 1970 \\
Grammatika & - Russkaja Grammatika, 2 Bd., \\
(Praha) 1979 & Praha, 1979 \\
PBulletinML & - The Prague Bulletin of \\
& Mathematical Linguistics \\
PStudiesML & - Prague Studies of Mathematical \\
& Linguistics \\
Slovar'(AN) & - Slovar' sovremennogo russkogo \\
(1950 ff) & literaturnogo jazyka, Moskva, \\
& 1950 ff. \\
VJa & - Voprosy jazykoznanija
\end{tabular}




\section{EINLEITUNG}

Es sind ungefähr 15 Jahre vergangen, seit im zuge der damals vorwiegend syntaktisch orientierten neueren Linguistik eine Monographie erschien, die den Adverbialen gewidmet war, die "Adverbialsyntax" von R. Steinitz (1971).

Das allgemein vielbeachtete Buch löste im besonderen in der europäischen Fachliteratur zu den Adverbialen unterschiedliche Resonanzen aus ${ }^{1}$; dennoch bleibt dem Problem der Adverbialen, im besonderen der freien ${ }^{2}$ - sieht man von Artikeln ab, die notwendigerweise auf Einzelaspekte beschränkt sind -, größeres Interesse versagt.

Zwei Gründe sind m.E. dafür evident: der eine hängt mit der Forschungsgeschichte der amerikanischen und westeuropäischen Linguistik zusammen, die die Kritik an der Syntaxforschung klassischer Chomsky'scher ${ }^{3}$ Prägung nicht konsequent zu einer Verbesserung dieser Theorie auswertete, sondern sich im wesentlichen ${ }_{4}$ einem anderen Problem zuwandte: der Erforschung der Semantik.

Der andere Grund hängt mit der Grammatiktheorie, dem Standardmodell, selbst zusammen, was z.B. in der Arbeit von Steinitz deutlich wird, deren Beschreibungsgrundlage das Standardmodell bildet: in der Syntaxtheorie Chomsky's ${ }_{5}$ und seiner Schule bleibt - auch nach dem Standardmodell ${ }^{5} 6^{-}$ unklar, wie die freien Adverbialen (im folgenden auch Advb ${ }^{6}$ ) $\mathrm{zu}$ beschreiben sind, wenn das Verhältnis von Form und Inhalt bei Dominanz der Form methodisch zum Angelpunkt der Beschreibung des Satzes deklariert wird. Ich werde auf diesen Punkt noch zurückkommen.

Eine konsequentere Entwicklung der Syntaxforschung in dem letzten Jahrzehnt ist m.E. in der osteuropäischen Linguistik zu beobachten, was, soweit mir zugänglich, im besonderen für die tschechische und die Linguistik der DDR gilt. Ich denke z.B. an Entwicklung und Ausarbeitung der "Funktional 
Generativen Beschreibung" (FGB) unter der Leitung von P. Sgall. Diese Entwicklung ist insofern konsequenter, als die Verf. mit der Theorie der FGB unter Berücksichtigung der formalen syntaktischen Relationen, nach denen modellhaft Sätze generiert werden können, versuchen, das von chomsky ausgeklammerte und von seinen schülern wie kritikern weitgehend vernachlässigte problem der Satzbedeutung in die Beschreibung miteinzubeziehen .

Das Modell der FGB halte ich für die russistische Linguistik auch deshalb für wegweisend, weil ihre Theorie, die bekanntlich den Anspruch erhebt, ein mit geordneten sprachlichen Ebenen operierendes umfassendes Sprachbeschreibungsmodell $2 u$ sein, an slavischen Sprachen, primär dem Tschechischen, daneben aber auch regelmäßig dem Russischen verifiziert wird.

Die Entwicklung der modernen Syntaxforschung in der DDR wird maßgeblich von den Arbeiten von R. Rưzicka und seiner Schüler bestimmt, die im Rahmen der linguistischen Konzeption der ehemaligen "Arbeitsstelle Strukturelle Grammatik" vergleichbare Positionen, wie sie von den tschechischen Linguisten vertreten werden, einnehmen. Für die vorliegende Untersuchung sind die Arbeiten von Růziłka auch deshalb bedeutsam, da er sich wiederholt - neuerdings Rủzicka (1980) mit den Adverbialen auseinandergesetzt hat, allerdings überwiegend in der Form des Adverbialpartizips. Die erwähnten tschechischen und deutschen Zentren linguistischer Forschung sind um so wichtiger, als sich die sowjetische Russistik formalen Syntaxbeschreibungen, zumal angewendet auf komplexe Strukturen - und als solche müssen Sätze mit Advb betrachtet werden - im großen und ganzen verschließt. Den so vielversprechend klingenden Aufgaben ${ }_{10}$ vor die sich die Erforschung der Adverbiale gestellt sah , folgte eine schon viele Jahre dauernde und im ganzen fruchtlose Diskussion über die "Determinanten" , so daß bei diesen Voraussetzungen 
erwartungsgemä $\beta$ keine neueren Arbeiten $z$ u verzeichnen sind, die dem gegenwärtigen internationalen Forschungsstand entsprechen

Ich habe mir die Aufgabe gestellt, die freien Adverbiale als Satzglieder $z u$ beschreiben und Regeln für ihre Verwendung in Sätzen zu formulieren.

Um dieser Aufgabe im Rahmen der vorliegenden Arbeit gerecht werden zu können, mußte ich Prioritäten bei der Behandlung des Themas setzen.

Das betrifft in erster Linie den Umfang der $z u$ behandelnden Adverbiale. Ich beschränke mich auf die "elementaren" Klassen der lokalen, temporalen und modalen Adverbiale; zum einen, weil ich meine, daß die sprachlichen Verhältnisse in diesen Klassen - primär der der lokalen und temporalen - "einfacher" sind als bei den abstrakten Relationen, die kausale, finale etc. Adverbiale beinhalten. Zum anderen, weil aus der Darstellung der "elementaren" Adverbiale m.E. methodische Einsichten auch für die Beschreibung komplexerer sprachlicher Fakten gewonnen werden können. So war es mir möglich, für spezifische Probleme, die die modalen Adverbiale aufwerfen, Lösungen vorzuschlagen. Ansonsten bin ich jedoch der Meinung, daß für die Darstellung der "abstrakten" Adverbiale eigene Untersuchungen erforderlich sind (s, dazu auch u. S. 46 f).

Des weiteren muß ich mich auf die mit dieser Fragestellung primär betroffenen linguistischen Bereiche syntax und Semantik beschränken und kann auf Probleme, die Grenzbereiche bzw. Nachbardisziplinen, vor allem Wotbildung und Prädikatenlogik implizieren, nur verweisen.

Aus den bisher vorliegenden Forschungsergebnissen $\mathrm{zu}$ den Adverbialen leite ich folgende Prämissen für den theoretischen Rahmen der Beschreibung ab: 
1. gefordert ist ein Satzverknüpfungsmodell;

2. die Beziehungen der zu verknüpfenden Sätze sind formal und semantisch bestimmt;

3. das sprachliche zeichen erfordert eine Beschreibung der Relation von Form und Inhalt.

Für die Lösung der hier gestellten Aufgabe erwiesen sich die bisher in vergleichbaren linguistischen Arbeiten praktizierten Untersuchungsmethoden als unbefriedigend. Die Behandlung der genannten drei Klassen von Adverbiale und der Versuch, sie als eine Klasse syntaktischer Ausdrücke zu definieren, erfordern eine Methodenvielfalt, insofern die syntaktischen und semantischen Merkmale der Adverbiale primär durch zugrundeliegende Prädikationen und Positionswerte im Satz bestimmt sind. Für die Beschreibung der Adverbiale als tiefenstrukturelle Prädikation werden Methoden herangezogen, die aus der generativen Transformationsgrammatik und aus prädikatenlogischen Analysen abgeleitet sind; für die Beschreibung des Positionswertes die Thema-Rhema-Analyse.

Es ist notwendig, die methodischen Schritte zu gewichten, da die beiden Faktoren von unterschiedlicher Relevanz bei der Verwendung der freien Adverbiale im Satz sind.

Bevor ich zu den Einzelanalysen komme (Kap. 3-5), möchte ich im folgenden Kapitel die Wahl der linguistischen Theorie und der Methoden für ihre Verifizierung begründen. 
2. ZUR METHODE

\subsection{ADVERBIALE IN NEUEREN SPRACHBESCHREIBUNGSMODELLEN}

Die bisher grobe Skizzierung des Forschungsstandes zu den Adverbialen soll durch ein paar Beispiele aktualisiert werden, die verdeutlichen mögen, daß die Beschreibung der Advb an das Problem des Verhältnisses von Form und Inhalt stößt und daß ihre Beschreibung Modelle, die diese beiden Seiten sprachlicher zeichen einander zuordnen, erfordert.

Sätze mit Advb sind in vielfacher Hinsicht mehrdeutig:

1. Formal homonym tragen die Lokaladverbiale in den folgenden Sätzen auf unterschiedliche weise zu der Satzbedeutung bei:

"Sie lernte Französisch in der Schule."

"Er fand die Mark in der Hosentasche."

Diese der Arbeit von Steinitz (1971, $30 \mathrm{ff}$ ) entnommenen Sätze lassen sich mühelos ins Russische übertragen und zeigen dann entsprechende sprachliche Erscheinungen, was darauf hindeutet, daß das problem - der Beitrag der Advb zur Satzbedeutung - ein übereinzelsprachliches ist:

(1) Ona izuxala francuzskij jazyk v skole.

(2) On našel monetu v karmane.

Unterschiedliche Lesarten in (3-4) und Mehrdeutigkeit in (57) sind durch andere Faktoren bedingt als bei dem Satzpaar $(1-2)$ :

(3) V sadu poet solovej.

(4) Solovej poet $\underline{v}$ sadu.

(5) Chleb $\underline{v}$ oblasti vpervye zagotovlen novymi kombajnami. 
(6) My priglasili vas $\underline{v}$ ijule prinjat' uXastie v konferencii.

(7) On Zenilsja po prikazaniju otca.

Entsprechende mehrdeutige Funktionen zeigen die Advb in folgenden deutschen Sätzen:

(8) Das Schema stellt den Verlauf im Raum dar. (Bierwisch, 1971, 16).

(9) ... erhob doch der französische Gesandte Benedetti in Ems, wo der greise König Wilhelm zu Kur weilte, trotz mehrfacher Abweisung in kränkender Weise neue Forderungen .

Neben dem Problem der syntaktischen Homonymie, das sich mit den Sätzen (3-9) stellt, werfen die folgenden Sätze die Frage nach syntaktischer Synonymie auf:

(10) $\mathrm{V}$ ètoj knige izlagaetsja istorija stolicy.

(11) Ėta kniga izlagaet istoriju stolicy. (zimmermann, 1978, 75)

(12) V sarae pachnet senom.

(13) Saraj pachnet senom ${ }^{2}$.

Wenn es richtig ist, das (1-13) typische strukturelle Eigenschaften von Sätzen mit Advb aufweisen, dann ist anzunehmen, das es sich dabei um Spezifika der freien Adverbiale handelt, die nicht für sätze mit obligatorischen Adverbialen (Adv) gelten, obwohl diese beiden Klassen von Satzgliedern mit identischen formalen Mitteln gebildet werden können, z.B. mit Adverbien oder mit PP. Und so wundert es denn auch nicht, daß in der Literatur der Frage nach Unterscheidungskriterien für $\mathrm{Adv}$ und Advb wiederholt Arbeiten gewidmet wurden. Bei unterschiedlichem Beschreibungsansatz: z.B. Valenzgrammatik, Standardmodell, FGB - stimmen die Antworten zu den Kernproblemen, die die Strukturbeschreibung von Sätzen mit Adv bestimmen, überein; in der Terminologie 
des Standardmodells ausgedrückt (S. 134 (52)): die Adv werden direkt von der "VP" dominiert, ihre Expandierungsregeln werden durch ihre Abhängigkeit von der strikten Subkategorisierung bestimm - soweit die syntaktische Beschreibung. Die konkrete Verwendung der Adv in Sätzen wird durch Merkmalmatrizes, die durch selektionsregeln gewonnen werden, geregelt und gehört damit ins Lexikon. Aus der Fachliteratur geht hervor, daß sich die Erforschung der Adverbiale primär den Aufgaben der Bestimmung der Unterscheidungskriterien für $A d v$ und Advb widmete und, darauf aufbauend, der Beschreibung der Adv selbst. Dagegen wurde der ausgesonderten Klasse der Advb weit weniger Beachtung geschenkt; bekanntlich ist sie im standardmodell (ibid.) innerhalb der Expandierungsregeln so eingeordnet, daß sie vom "Prädikatskomplex" dominiert wird, womit aber Kernprobleme, die die Advb stellen und die z.B. auch Steinitz illustrierte (s.o. die Sätze (1-2)), offen bleiben.

Man kann also feststellen, daß die Klasse der Advb im wesentlichen negativ in ihrem Verhältnis und im Unterschied zu Adv beschrieben ist. Die vorliegende Arbeit will den Versuch machen, die Klasse der Advb als Satzglieder zu beschreiben und Regeln, die ihre Verwendung bestimmen, zu formulieren. Ich beschränke mich dabei

- auf die russische Literatursprache der Gegenwart und

- auf Adverbiale in der Form von Präpositionalphrasen . Dabei kann die Frage der Topologie der Präpositionen ausgeklammert werden, da sie in Zusammenhang mit Adv abzuhandeln ist, worüber auch für das Russische Ergebnisse vorliegen ;

- auf die drei elementaren Adverbialklassen: lokal, temporal, modal.

Für die Erarbeitung des Beschreibungsansatzes habe ich die Literatur zu den erwähnten zwei Problemkreisen - Aufstellung von Unterscheidungskriterien zwischen $\mathrm{Adv}$ und $\mathrm{Advb}$ und Beschreibung der Adv ausgewertet und habe, ausgehend von dieser Basis, die im wesentlichen negative Bestimmungen für 
Advb liefert, positive Argumente für meine Aufgabe zu ermitteln versucht.

Dabei wurde klar, daß für diese Beschreibung ein Modell gebraucht wird, das Satzverknüpfungen darstellt. Mit diesem Modell müssen auch - wenn sich die Darstellung nicht auf pauschale Aussagen über die Relation der $z u$ verknüpfenden Einheiten beschränken soll - die spezifischen Verknüpfungen, die durch adverbiale PP ausgedrückt werden, beschreibbar sein.

Die Lösung dieser Aufgabe erfordert m.E. ein Modell, das die aus empirischen Untersuchungen gewonnenen Einheiten der Satzsyntax und der Satzsemantik einander zuordnet.

Die folgende kritische würdigung von Untersuchungen $z u$ Adverbialen dient der Begründung der wahl des im weiteren praktizierten Beschreibungsmodells und der aus dieser Hypothese sich ergebenden Untersuchungsverfahren an empirischem Material. Bei der Auswahl der Arbeiten ließ ich mich von folgenden Kriterien leiten:

- die Beschreibungsansätze der Arbeiten müssen ineinander übersetzbar sein;

- die Arbeiten sollen die Entwicklung der Forschungsergebnisse und - soweit mir bekannt - den aktuellen Stand dokumentieren;

- sie sollen nach Möglichkeit die Erforschung auch russischen Sprachmaterials enthalten. 
2.1.1 ADVERBIALE IN EINEM MODELL MIT TRANSFORMATIONEN NACH HARRIS

Johnson (1970) hat mit seiner Arbeit über $\left\{\begin{array}{c}N^{1} \\ A \\ V\end{array}\right\}$ ot $N^{2}$

Konstruktionen für das Russische eine Pionierarbeit geleistet, indem er an einem Korpus von ca. 1.100 Belegen nachweist, daß man bei Anwendung formaler linguistischer Analyseverfahren einem Grobteil homonymer, syntaktischer Strukturen verschiedene strukturbeschreibungen geben und so dank einer stärkeren Generalisierung eine fundiertere Rlassifikation ableiten kann, als sie in den üblichen standardwerken geboten wird.

Die nun folgende kritische Auseinandersetzung mit Johnson soll den Nachweis führen, an welchen Punkten sein unbestritten fruchtbarer theoretischer und methodischer Ansatz erweiterungsbedürftig ist, und es soll ausgeführt werden, aufgrund welcher linguistischer Theorien weiterführende methodische Verfahren zur Beschreibung von PP entwickelt worden sind bzw. werden können. Die Listen von Transformationen (Tf), die Johnson erstellt, sind eine Anwendung des Harris'schen "morphophonemic change" operators.

Bekanntlich sind die Harris'schen Tf dadurch gekennzeichnet, daB mit ihnen an Basisstrukturen bestimmte operationen durchgeführt werden, durch die die Basisstrukturen in komplexere Strukturen überführt werden, oder anders gesagt, die Beziehungen zwischen komplexen strukturen und Basisstrukturen beschrieben werden. Die Frage der Bedeutungsinvarianz bei oberflächenstruktureller Formvarianz wird von Harris und bei der Rezeption seiner Theorie nicht einheitlich behandelt. Wie im folgenden noch gezeigt wird, ist es diese Frage, erweitert durch die generative Ronzeption einer Tiefenstruktur, um deren Lösung sich die neuere und neueste Linguistik zur Beschreibung von Sätzen mit Hilfe unterschiedlicher theoretischer Ansätze bemüht. 
Die Tf bei Johnson sind formal einheitlich "intra-class" und vor allem "inter-class" Tf (Definition s. Johnson, 38). Bei der Anwendung seiner histen von Tf muB Johnson selbst die Beobachtung machen, das diese Art von Tf für die Ronstruktionen $\mathrm{N}^{1}$ ot $\mathrm{N}^{2}$ und $A$ ot $\mathrm{N}^{2} \mathrm{zu}$ eindeutigeren strukturbeschreibungen und damit Rlassifikationsmerkmalen führt als für die Ronstruktion $\mathrm{V}$ ot $\mathrm{N}^{2}$ :

"It will be noted that the analysis of the verbal constructions is somewhat less effective than that of the nominal units in isolating semantic subgroups and providing them with formal specifications" (186).

Johnson sieht den Grund dafür in folgenden zwei Tatsachen:

1. Die russischen Verbwurzeln unterliegen nicht in dem Maße morphologischen Ableitungsregeln wie die Nomina.

2. Die grammatische und semantische Struktur des russischen Verbs ist weitaus komlexer und weniger klar als die des Nomens (186 f).

Mir scheint, daß diese Begründung für die nicht befriedigende Adäquatheit der von Johnson verwendeten Klassen von Tf für Ausdrücke, bestehend aus Verb + Präpositon + komen, nicht ausreicht, und zwar aus folgenden zwei Gründen:

1. Verschafft man sich einen tberblick über die transformationellen Eigenschaften $\operatorname{der} \mathrm{N}^{1}$ ot $\mathrm{N}^{2}$ und $A$ ot $\mathrm{N}^{2}-$ Konstruktionen, dargestellt in den Beispiellisten der Tabellen 1 bis 9 (summary, 78), so fällt auf, daß mit Ausnahme der Beispiele der Gruppen 7,8 und 9 die überwiegende Mehrzahl der Beispiele transformationelle Beziehungen zu verbalen Konstruktionen aufweist (neben solchen $z \mathbf{u}$ anderen, versteht sich). Die Beispiele der Listen 7, 8 und 9 gehören zu der üblicherweise als attributive, nominale Ergänzung bezeichneten Rlasse: 
kljuc ot biblioteki; korobka ot botinok; setka ot komarov (78).

Diese Beobachtung entgeht Johnson nicht. "The nominal structures are, to a much greater extent than their verbal counterparts, derived. These considerations mean that the $\mathrm{V}$ ot $N$ constructional type, or at least some substantial part of it, forms the kernel structure from which the remaining ot usages are derived by means of various transformations" (124).

Johnson stellt jedoch diese Beobachtungen, wie bereits erwähnt, in den Erklärungszusammenhang der morphologischen Ableitungsmöglichkeiten des russischen Verbs. Die Forschungsergebnisse der seit dem Erscheinen der Arbeit von Johnson vergangenen 15 Jahre $2 \mathrm{u}$ präpositionellen Phrasen, wie aber auch die unbefriedigenden Analyseergebniss von $\mathrm{V}$ ot $\mathrm{N}^{2}-$ Konstruktionen in der Arbeit von Johnson legen einen anderen Erklärungszusammenhang nahe. Damit komme ich zu dem zweiten Grund, den ich an Beispielen, die unter der semantischen Beschreibung "causal" zusammengefaßt sind, demonstrieren möchte.

2. Diese Gruppe stellt eine umfangreiche Liste von Verben dar (s. S. 138-152). Bei Anwendung der von Johnson zugrundegelegten Transformationskriterien bieten sich hier außerdem weitere Tf an:

"(19) Zena mlela ot voschiక̌xenija"

Tf A: そena mlela, buduci voschišennoj

Tf B: Zena, voschiక̌tennaja, mlela

"(22) Zenšciny onemeli ot izumlenija"

Tf A: zenšxiny onemeli, buduxi izumlennymi

Tf B: Zenšiny, izumlennye, onemeli 
"(27) kinžal zaržavel ot krovi"

Tf A: kinžal zaržavel, buduči okrovavlennym

Tf B: kinžal, okrovavlennyj, zaržavel

"(36) ona otupela ot sna"

Tf A: ona, buduci sonnoj, otupela

Tf B: ona, sonnaja, otupela

Die Beispiele mögen genügen, um darzulegen, daß zwischen den Sätzen transformationelle Beziehungen folgender Art vorliegen:

$\mathrm{NP}^{\mathrm{I}} \mathrm{VP}$ ot $\mathrm{NP}^{2} \stackrel{\mathrm{Tf}^{\mathrm{I}}}{\Longrightarrow} \mathrm{NP}^{1} \mathrm{VP}$ buduci $\left\{\begin{array}{c}\text { Part } \\ \mathrm{A}\end{array}\right\}^{\mathrm{NP}}$

$$
\stackrel{\mathrm{Tf}^{2}}{\Longrightarrow} \mathrm{NP}^{\mathrm{I}} \quad\left\{\begin{array}{c}
\mathrm{Par} t \\
\mathrm{~A}
\end{array}\right\}^{\mathrm{NP}} \quad \mathrm{VP}
$$

Damit ist klar, daß "ot $\mathrm{N}^{2}$ transformationell in Relation $\mathrm{zu}$ einem Prädikat, dem zweiten Prädikat des $z u$ analysierenden Satzes steht. Diese Tf, die übrigens auch auf Harris und zwar auf seinem Typ der "sentence connective ( $\left.\phi_{c}\right)$ " Operation fußen, die aber bei Johnson nicht zur Anwendung kommen, erweitern und verändern die linguistische Analyse mit Hilfe von Tf in mindestens einem entscheidenden punkt: die PPAnalyse von $\mathrm{V}$ ot $\mathrm{N}^{2}$-Konstruktionen gibt Argumente dafür, die bei Johnson sich linear auf einer Ebene, der morphosyntaktischen, abspielenden intraclass- und interclass-Tf durch ein Modell zu ergänzen, das hierarchische Beziehungen für die Ableitung von Sätzen ansetzt; z.B. die Derivationsgeschichte von Sätzen $z$ u modellieren, wie sie generative Modelle vorschlagen. 


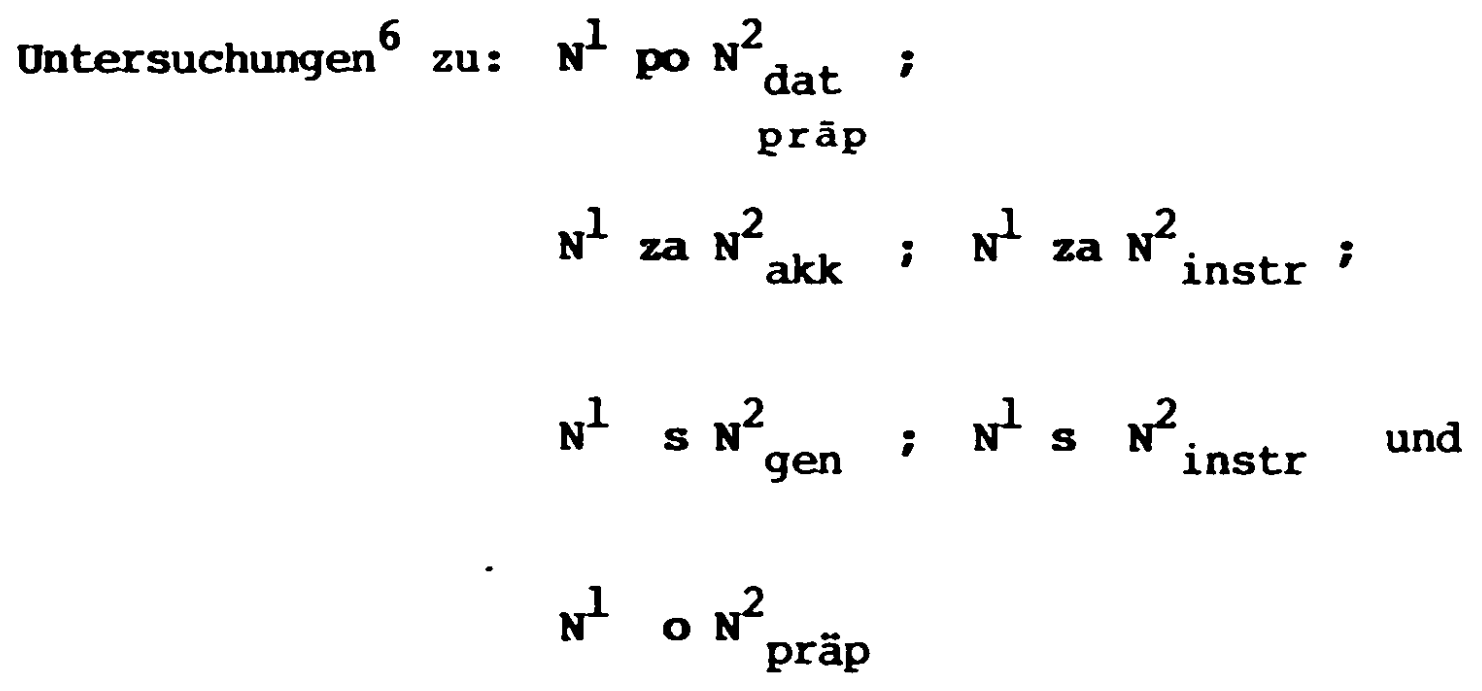

bestätigen die Ergebnisse von Johnson $z u N^{1}$ ot $N^{2}$ in einem, wie mir scheint, zentralen Punkt: eine Klasse, und sie umfast die überwiegende Mehrzahl der Beispiele dieser nominalen Ronstruktionen, hat in der definierten weise transformationelle Beziehungen $z \mathrm{u}$ entsprechenden verbalen Ronstruktionen; eine zweite Klasse $z u$ nominalen Konstruktionen, im besonderen $z u N^{1} \mathbf{N}^{2}$ gen ( $s$. dazu auch Johnson 78, Tabelle 9), d.h. zu attributiven nominalen Ergänzungen. Diese letzteren präpositionalen Konstruktionen klammere ich wie bereits erwähnt - im weiteren aus meiner Darstellung aus.

Zieht man die Ergebnisse von Johnson zu A ot $\mathrm{N}^{2}$ in Betracht, daß nämlich auch diese Ronstruktion in transformationeller Beziehung zu verbalen Konstruktionen steht (s. Johnson, 91, Pl bis P6), so ist auch sie mit dem Problemkreis der präpositionalen Ronstruktionen vom Typ ( $V$ Präp $N$ ) erfast.

Die Analyse homonymer NPN-Ronstruktionen mit Hilfe von intraclass- und interclass-Tf hat im besonderen formale Unterscheidungskriterien für attributive PP einerseits und adverbiale PP andererseits geliefert sowie formale Beziehungen zu synonymen Ronstruktionen innerhalb dieser zwei Klassen aufgedeckt. Eine Unterscheidung der Ronstruktion (V Präp $N^{2}$ ) in Präpositionalobjekte und präpositionale Adverbiale wird bei Johnson - wohl in Folge der erwähnten 
methodischen Einschränkungen - nicht vorgenommen; es scheint,

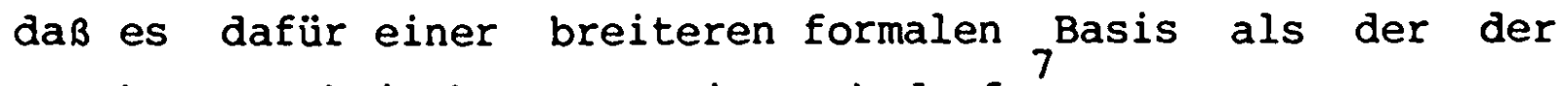
morpho-syntaktischen Operationen bedarf.

Abschließend kann festgehalten werden, daß die kritische Analyse von Johnson ergab, daß für eine PP-Analyse die linear ablaufenden Tf einer Ergänzung bedürfen, und zwar durch hierarchisch geordnete Tf innerhalb eines generativen Modells. 


\subsubsection{ADVERBIALE IN EINEM MODELL NACH CHOMSKY'S "ASPEKTE DER SYNTAXTHEORIE"}

Die Idee von hierarchischen Beziehungen zwischen den Gliedern des Satzes findet man z.B. im Standardmodell. Mit dessen Anwendung auf die präpositionalen Konstruktionen hat Chomsky die neuere Diskussion dazu - ob positiv oder negativ entscheidend bestimnt.

Eine Anwendung des Chomsky'schen Standardmodells auf Adverbialkonstruktionen des Deutschen hat R. Steinitz (1971) unternommen. Die Adverbiale werden hinsichtlich ihrer positiven bzw. negativen Eigenschaft, das verb zu subkategorisieren, klassifiziert.

Mit Hilfe eines auf Tf, Subkategorisierungs- und Selektionsregeln aufgebauten Beschreibungsapparates werden Kriterien für die Unterscheidung gewonnen (Steinitz, 1971, $40 \mathrm{f}$ ). Das Modell sieht damit einen einheitlichen formalen Beschreibungsrahmen für die Klassifizierung von attributiven PP, Präpositionalobjekten und adverbialen PP vor und wendet im besonderen Paraphrasen für die Subklassifizierung der Adverbiale an.

Im folgenden sollen - im Hinblick auf die hier primär interessierende Frage der Beschreibung der Advb - die Ergebnisse bei Steinitz für die lokalen Adverbiale im Deutschen diskutiert werden.

Für die lokalen Adverbiale lassen sich die beiden folgenden Hauptklassen nachweisen:

1. die "Quasi-lokalen" Adverbiale (im folgenden Advb. ; Steinitz $34 \mathrm{f})$, die freie fakultative Ergänzungen im Satz sind und Vb nicht kategorisieren. Sie lassen sich durch $T f$ auf Sätze ("Paraphrasen") verschiedener Art zurückführen, 
2.B. auf einen temporalen Nebensatz - vgl, zu den O. (S. 20) angeführten Beispielen:

"(49) Als sie in der Schule war, lernte sie Französisch" (loc. cit. 30); dagegen:

"(53) Er fand die Mark, als sie in der Hosentasche war (ibid.); eher:

"(57) Er fand die Mark. Sie war in der Hosentasche" (ibid).

Die Erklärung für die Beziehung zwischen den so gewonnenen Sätzen bleibt provisorisch, nämlich als Satz, "der dem Matrixsatz nebengeordnet ist" (35). Diese von Steinitz selbst als unzureichend bewertete Beschreibung ist eine Folge des mit dem Standardmodell konzipierten theoretischen Rahmens (ausführlicher dazu u. Kap. 3.1.2, s. $71 \mathrm{ff}$ ).

Die nicht ausreichende Grundlage für die Paraphrasierung von Advb wird noch deutlicher bei der 2. Klasse, die ihrerseits in zwei Klassen zerfällt:

2. die lokalen Adverbiale, die enger mit dem verb in Verbindung stehen; dabei werden unterschieden:

2.1 die obligatorischen Ergänzungen (im folgenden Adv.) in Sätzen wie:

"(33) Peter sitzt in der Wanne.

(32) Peter und Mariechen kommen noch her" (loc. cit. 21).

Die Merkmale der strikten Subkategorisierung sind bei Verben wie sitzen und kommen nach Steinitz ( $14 \mathrm{f})$ :

$\left(\right.$ sitzen,$\left[+\mathrm{v}, \ldots+\right.$ Adv $\left.{ }_{\text {Loc }}[\ldots . .].\right)$

Entsprechend kann eine bestimmte Menge von Verben klassifiziert werden (13). Eine weitergehende Analyse mit Hilfe von 
Selektionsregeln ermöglicht eine Subkategorisierung der Präpositionen ( $86 \mathrm{ff})$.

Unterschieden von dieser Klasse der Adv werden 2.2 Ergänzungen - im folgenden $A_{d v b_{2}}$ - in Sätzen wie:

"(100) Er arbeitet in der Universität" (10c. cit. 56).

"(102) Die Kinder spielen im Garten" (loc. cit. 57).

Die Diskussion zu diesen Sätzen verläuft nicht ohne Widersprüche zu der Gesamtdarstellung - darüber ist sich die verf. im klaren. Ihre Lösungsversuche unter Berücksichtigung von Faktoren der aktuellen Satzperspektive einerseits und andererseits der Möglichkeit bzw. Blockierung der Negation 10 von VP und PP (loc. cit. $56 \mathrm{f}$ ), können den Einwand nicht entkräften, daß die vorgenommene Klassifizierung von lokalen Adverbialen infolge der drei verschiedenen Mengen von Kriterien nicht distinkte Klassen ergibt: Einerseits sind in (100) und (102) die lokalen Adverbiale für Vb nicht obligatorisch, der Eliminierungstest ergibt korrekte Sätze:

Er arbeitet.

Die Rinder spielen.

Andererseits lassen sie sich im Rahmen des von Steinitz angewendeten Grammatikmodells nicht sinnvoll zu Sätzen paraphrasieren. Die Verf. trägt dieser gewissen Ungenauigkeit des Beschreibungsmodells Rechnung, wenn sie feststellt: "I m g a $n z$ e $n$ (gesperrt H.S.) stimmt also die Möglichkeit und Unmöglichkeit, adverbiale Subklassen als sätze zu : realisieren, mit der verteilung dieser Subklassen auf die Kategorie Advb ${ }_{1}$ und $A_{2 v b}$ überein: Adverbiale von $A d v b_{1}$ können zu Adverbialsätzen weiterentwickelt werden, Adverbiale von Advb $_{2}$ können das nicht" $(60)$.

Zusammenfassend kann man $z u$ der Arbeit von Steinitz feststellen, daß das standardmodell ein geeigneter theoretischer Rahmen für die Beschreibung der Klasse der Adv ist; was 
dagegen die Klasse der Advb betrifft, so liegen dazu zwei Schlußfolgerungen nahe: zum einen liefern - abgeleitet aus dem generativen Modell - die Regeln der strikten Subkategorisierung die Kriterien für die Unterscheidung der beiden Klassen Adv und Advb in dem Sinn, daß sie nur für Adv gelten; die Advb untersucht die verf. auf der Basis der im Standardmodell vorgetragenen Hypothese (2.B. 271, Anm. 28), daß die Adverbiale "aus ganzen Sätzen transformiert" seien (272); jedoch befriedigen die Paraphrasen, die Steinitz als Untersuchungsprozedur für die Derivation von der Oberflächenstruktur "PP" zu der Tiefenstruktur "\#S\#" entwickelt, nicht. Die Folge davon ist, daß die zweiteilung der Advb in der vorgeschlagenen Klassifizierung nicht überzeugt und zweifel aufkommen, ob sie sich durch aktuelle Satzperspektive, Betonungsverhältnisse und Negation-Tf motivieren läßt.

Es fällt auf, daß sich mit den Beschreibungsprozeduren, die aus dem Standardmodell abgeleitet sind, die Hypothese, Advb seien auf Sätze zurückzuführen, weder verifizieren noch falsifizieren läßt.

Aus den Fragen, die bei der Beschreibung der freien Adverbiale mit dem im Standardmodell entwickelten Regelmechanismus offen bleiben, folgt als erste Forderung an ein verbessertes Modell, den Begriff der Paraphrase präziser zu definieren. Eine solche weiterführende Präzisierung scheint mir das Modell der "Funktional Generativen Beschreibung" zu enthalten.

Im folgenden soll unter dem hier interessierenden Gesichtspunkt - der Einordnung der Advb in ein Sprachbeschreibungsmodell - der theoretische Rahmen der FGB charakterisiert werden. Die Konzeption der Relation zwischen Form und Funktion, wie sie das Modell der FGB expliziert, schafft die Voraussetzungen dafür, daß die FGB anstelle des für die Beschreibung der Advb unscharfen Begriffs der "Paraphrase" den, wie ich unten ausführen werde, präziseren Begriff der "grammatischen synonymie" verwendet. 
2.1.3 ADVERBIALE IN DER "FUNKTIONAL GENERATIVEN BESCHREIBUNG" (FGB) (PRAG)

Ausarbeitung und Uberprüfung des Modells der FGB an empirischem Sprachmaterial werden von inzwischen mehr als fünfzehn Jahre dauernden Auseinandersetzungen mit anderen linguistischen Theorien begleitet. Im besonderen wird die Frage diskutiert, was leistet eine Dependenzgrammatik, zu der die Autoren der FGB ihr Modell zählen, im Unterschied und evtl. mehr ${ }_{1}$ als die generative Transformationsgrammatik Chomsky's.

Für die Autoren der FGB gilt als unbestritten, daß die von Chomsky erhobenen Forderungen an ein linguistisches Modell von fundamentaler Bedeutung für jede linguistische Theorie sind; so werden diese von ihnen übernommen: Das Modell soll

1. die Menge der grammatischen Sätze definieren und soll

2. den einzelnen generierten Sätzen geeignete strukturelle Charakteristiken zuschreiben (Sgall, 1975, 60).

Kontrovers sind dagegen die Standpunkte $z \mathbf{u}$ der Frage der Behandlung der Semantik in diesem Modell. Als Mängel der Konzeption in dem Standardmodell wie auch derjenigen in den nachfolgenden Arbeiten von Katz und im weiteren der der generativen Semantiker betrachten die Autoren der FGB Folgendes:

1. Die semantische Interpretation bezieht sich auf lexikalische Einheiten und

2. - konsequenterweise - werden Syntax und Semantik "als zwei verschiedene Komponenten des Systems der Sprache beschrieben, die nur als Ganzes verknüpft werden. Wie es in der traditionellen linguistischen Semantik oft üblich war, geht auch diese Konzeption von den wortbedeutungen (genauer - von den Bedeutungen der Formative) aus" (Sgall, 1967, 18f).

Dem problematischen Verhältnis von Form und Funktion (Prager Schule) oder Ausdrucksform und Inhaltsseite (Hjelmslev) 
versucht die FGB dadurch Rechnung zu tragen, daß in ihr "die semantische Interpretation ihre eigene hierarchische syntaktische Struktur hat, die nicht allgemein mit der cohne Rücksicht auf Semantik gewonnenen) Satzstruktur übereinstimmt. Anders gesagt, es sollen nicht nur wortbedeutungen (und ihre Verkettungen, oder die Amalgame) gewonnen werden, sondern auch syntaktische Beziehungen sollen semantisch interpretiert werden" (ibid. $21 \mathrm{f}$ ). Die Bemühungen um die Einordnung der Semantik in eine formale Beschreibung natürlicher Sprachen haben nach den Autoren der FGB dazu geführt, daß sich die zunächst verschiedenen linguistischen Richtungen von Transformationsgrammatik einerseits und Dependenz-bzw. Stratifikationsgrammatik andererseits einander angenähert haben. In der Frage nach dem eigentlichen ziel der Linguistik "We find an agreement - perhaps unexpected but non wholly accidental - between various trends: be it Hjelmslev's relationship of content and expression, Prague functional approach, or formulations as

"To understand the ability of natural languages

to serve as instrument to the communication of thoughts and ideas we must understand what it is that permits those who speak them consistently to connect the right sounds with the right meanings." (Katz, 1966, p. 100)

- cf. Chomsky, 1968b, p. 15; Lakoff, 1969, p. 117 - they show that the proper task of linguistics consists in the description (and/or explanation) of the relation between the set of the semantic representations and that of the phonetic forms of utterances" (Sgall, Hajicová, 1973, 2 f).

Für eine Einordnung der Semantik in eine linguistische Theorie bedurfte es nicht nur der Klarstellung des Verhältnisses von Semantik und Syntax und dessen Beschreibung, sondern auch einer Grenzziehung zwischen linguistischer Bedeutung und faktischem wissen einerseits wie auch ontologischem Inhalt andererseits. 
Gegen die Ubernahme von Positionen aus der logischen Semantik in die Grammatik, wie sie bei gewissen Strömungen der TG in der Lehre von den sprachlichen Universalien $z$ verzeichnen ist, machen die Autoren der FGB geltend, daß ihr Modell ein linguistisches ist in seiner zielsetzung und seinen Analyseverfahren. Andererseits wird die Logik $z u$ den Nachbarwissenschaften gezählt, mit denen die Linguistik gerade auf dem Gebiet der Semantik existentielle Berührungspunkte hat. Deshalb lehnen die Autoren der FGB auch den orthodoxen standpunkt des strukturalismuns $a b$, der "meaning" auf linguistische Daten beschränkt. Ihre Position kommt in folgender Stellungnahme zum Ausdruck:

"We consider it useful to avoid both extreme standpoints in these questions. Liguistic meaning is not identical with the cognitive content and if it is to be studied by linguistic means, structural features of individual languages must be taken into account" (loc. cit. 20).

Für das Modell der FGB waren Erkenntnisse

1. über den "asymmetrischen Dualismus" (Skalička, 1935) des sprachlichen Zeichens und

2. über die "komplex hierarchisierte struktur der natürlichen Sprache" leitend (Sgall, 1975, 62).

In dem Modell der FGB werden auf fünf Ebenen - der tektogrammatischen, phänogrammatischen, morphematischen, morphologischen und phonetischen - Relationen von Form und Funktion angesetzt und an empirischem sprachmaterial erforscht. Die höchste Ebene, die tektogrammatische (im folgenen T-Ebene), generiert Repräsentationen von Sätzen, aller Sätze, die mit dem Modell beschrieben werden können. Auf dieser Ebene werden die semantischen Relationen angesetzt. Sie erscheinen in der formalen Beschreibung als Funktoren, deren Anzahl relativ eng begrenzt gehalten wird und die den semantischen Satzgliedern entsprechen. Sie sind aus der Erforschung der Satzgliedebene gewonnen. Ihre 
verschiedenen Typen entsprechen verschiedenen Abhängigkeitsrelationen zwischen ihren Aktanten, den Satzgliedern der Satzgliedebene; die Relationen sind binär. Als Funktoren sind auf dieser Ebene der semantischen Relationen auch die adverbialen Ergänzungen $\left(R_{d}\right)$ repräsentiert (Explizite Beschreibung, II, 1975, 20 f).

Die allgemein in der Literatur geforderte Unterscheidung zwischen obligatorischen und fakultativen adverbialen Ergänzungen wird insoweit methodisch präziser definiert, als ein Test - der Dialog-Test (Panevová, 1978b, 227 f; Beranová, 1975) - entwickelt wurde, der auch in dieser Arbeit als Ergänzung der Subkategorisierungsregeln, soweit die Untersuchungen das Problem von Unterscheidungskriterien für obligatorische und fakultative Adverbiale berühren, Verwendung findet (s.u. S. $56 \mathrm{f}$ ).

Der Dialog-Test erweist sich für die Lösung der Aufgabe als ein besonders geeignetes Unterscheidungskriterium, weil damit nicht nur das sprachliche System, sondern auch die konkreten sprachlichen Außerungen untersucht werden können.

Der Einbeziehung der Bedeutungsstrukturen - was für die TEbene heibt: die Satzsemantik - in das Sprachbeschreibungsmodell ging eine intensive Beschäftigung mit der Frage, was für die Linguistik unter Bedeutung $z u$ verstehen sei, voraus. Es wurde schon die Forderung nach einer Grenzziehung zwischen Tatsachenwissen, kognitivem Inhalt und sprachlicher Bedeutung erwähnt. Bei den Untersuchungen dazu erwies sich die Grenzziehung zwischen kognitivem Inhalt und sprachlicher Bedeutung (linguistic meaning) als diffizil. Eines der zentralen Probleme bleibt die Bestimung der grammatischen Synonymie, zu dem über mehrere Jahre hinweg wiederholt Untersuchungen publiziert wurden (Vgl. Panevová, 1973a; Sgall, Hajicová, Procházka, 1977; Sgall, Hajičová, Panevová, 1986). 
Mit der Ausarbeitung dieser theoretischen Positionen war eine Präzisierung auch der linguistischen Untersuchungsmethoden verbunden: Zur Vermeidung subjektiver Schlüsse über die Bedeutungsstrukturen wurden operationelle Tests entwickelt (z.B. Dialog-Test, Frage-Test, Negations-Test), mit denen die Ausdrucksformen untersucht werden, um ihre Funktionen $z u$ ermitteln und synonyme und homonyme Strukturen $z u$ ordnen. In "Linguistic Semantics and Intensional Structure" (Sgall, hajiČvá, Procházka, 1977) konzentriert sich die Auseinandersetzung auf die Bestimmung der sprachlichen Bedeutung und daraus resultierend der grammatischen synonymie im Unterschied zu Definitionen der Bedeutung der Logik. Die Autoren kommen zu dem Schluß, daß für die Beschreibung der sprachlichen Strukturen es notwendig ist, unterschiedliche "Grade der Synonymie" (loc. cit. 187) zu unterscheiden. In Satzpaaren mit z.B. konversen Prädikaten liegt keine Synonymie in "sensu strico" vor, obwohl die Ausdrücke eine identische intensionale struktur haben. Für die sprachliche Bedeutung gilt es jedoch, das "surplus", "die Domäne der linguistischen Bedeutung" (ibid. 188), zu erkennen, das in diesem Fall mit dem "Agens" gegeben ist und in solchen Satzpaaren durch unterschiedliche lexikalische Einheiten repräsentiert wird. Der Grad der Synonymie von Ausdrücken wird an der grammatischen Synonymie in "sensu stricto" gemessen.

Sie liegt vor, wenn Ausdrucksformen folgende Kriterien aufweisen (Panenová, 1973a, 133):

Sie unterscheiden sich nicht im Hinblick auf:

1. Ihre "lexikalischen Einheiten auf jeder Ebene"; d.h. sie müssen identische Lexeme beinhalten (besondere Regelungen sind für Wortbildungsregeln zu treffen):

2. "Die Abhängigkeitsrelation zwischen den Elementen (Wortformen) in der Konstruktion auf jeder Ebene";

3. "Die grammatischen Bedeutungen (d.h. weder Funktoren noch Grammateme dürfen auf der $T$-Ebene verschieden sein)".

4. Die Thema-Rhema-Gliederung. 
Mit dieser Definition gibt das Modell der FGB dem Begriff der grammatischen Synonymie einen relativ engen Anwedungsbereich - einen engeren, als es z.B. im Konzept von Saumjan/Soboleva (loc. cit. 129) oder in dem von Mel'cuk/Žolkovskij der Fall ist (Bémová, Weisheitelová, 1975).

Die folgende Darstellung der Bedeutungsstrukturen in Sätzen mit freien Adverbialen stützt sich auf die in der FGB ausgearbeitete Definiton der grammatischen Synonymie. Um die mit den einführenden Beispielen (z.B. 1-2) aufgeworfenen Fragen wiederaufzunehmen, können wir jetzt feststellen, daß mit der FGB ein Beschreibungszusammenhang gegeben ist, aus dem folgt, daß der temporale NS nicht als die TS der fakultativen Lokaladverbiale in (1-2) gelten kann.

Wenn wir zu unseren im Hinblick auf die Advb gestellten Fragen die Ergebnisse der FGB zusammenfassen, so können wir feststellen:

1. die Adverbiale werden auf der T-Ebene, der Ebene der semantischen Relationen, als Funktoren beschrieben;

2. für die Untersuchung ihrer Ausdrucksformen wurden operationelle Tests entwickelt, die auf dem Hintergrund der Definiton der sprachlichen Bedeutung konzipiert wurden;

3. der Dialog-Test ist ein Kriterium für die Beschreibung der obligatorischen Adverbiale;

4. spezielle operationelle Tests für die Beschreibung der fakultativen Adverbiale sind nicht entwickelt. Jedoch läßt sich die Methode zur Gewinnung von operationellen Tests für unsere problemstellung nutzen; außerdem enthalten die zahlreichen empirischen Untersuchungen $z u$ freien Adverbialen, die in den speziellen Kapiteln dieser Arbeit zur Sprache kommen werden, für die hier gestellte Aufgabe wertvolle Ergebnisse und Hinweise. 
2.1.4 DIE "ADVERBIALSEMANTIK" VON R. BARTSCH

Eine Untersuchung der Satzsemantik der Adverbiale, bei der auch Tests ihre Anwendung finden, enthält das Buch von $R$. Bartsch "Adverbialsemantik" (1972). Ich referiere darüber im folgenden, insoweit sich diese Ergebnisse für das hier behandelte Thema auswerten lassen.

Die recht allgemeine Einordnung der Advb im Standardmodell: "Ubrigens sind viele Adverbiale der Art und Weise, wie eine Reihe anderer Adverbiale aus ganzen Sätzen transformiert unter Tilgung des Subjekts" (271 f, Anm. 28) finden wir z.B. bei Lakoff wiederaufgenommen, jedoch weiterentwickelt und präzisiert, wobei er Ergebnisse der logischen Beschreibung von Sprache übernommen hat. "Es wurde von Reichenbach und in jüngster Zeit von Parsons vorgeschlagen, daß Adverbien der Art und Weise wie carefully (sorgfältig) Operatoren sind, die ein Prädikat auf ein anderes abbilden" (Lakoff, 1971, 122). Die Arbeit von R. Bartsch ist ein Versuch, Adverbiale der natürlichen Sprache mit Hilfe von Klassifizierungen, die aus der Prädikatenlogik gewonnen wurden, zu beschreiben und Regeln für ihre verwendung und Kombinationsfähigkeit im Oberflächensatz $\mathrm{zu}$ formulieren. In seiner ausführlichen Rezension "Pseudologik und Natürliche Generative Grammatik Anmerkungen zur 'Adverbialsemantik' von Renate Bartsch" (Schwarze, 1976), bezieht Schwarze die Position eines scharfen Kritikers, wenn er Bartsch vorwirft, sie arbeite mit logischen Entitäten, Grundbereichen, die durch Klassen von Individuenvariablen definiert werden, für die es keine gesicherten logischen Erkenntnisse gebe $\left(\begin{array}{ll}76 & \mathrm{f}\end{array}\right)$. Was die Beschreibung der Objektsprache angeht, kann Schwarze dennoch nicht umhin anzuerkennen, daß es Bartsch gelingt, "mit großem sprachlichen Feingefühl, sich im sumpf der traditionell 'Adverbien' genannten Ausdrücken zumindest zu orientieren" (105). 
Bartsch rechtfertigt Methode und Inhalte ihrer Analyse und zeigt auf die offenen Fragen, die in ausschlieblich oder primär linguistisch fundierten Arbeiten $z u$ bemängeln sind, wenn sie über ihre Arbeit schreibt: "Vielmehr werden im Zusammenhang mit Problemen der Adverbialsemantik - die zum Teil auch Probleme der Nominalisierungstransformationen sind - über die in der Prädikatenlogik üblichen Individuenvariablen hinausgehend verschiedene Typen von Individuenvariablen eingeführt. Auf diese Weise werden grobe Mängel der angeblich prädikatenlogischen Darstellungen des generativen Semantikers G. Lakoff überwunden ${ }^{5}$, die darin bestehen, daß er stets ganz undifferenziert das satz-symbol $S$ einbettet, wodurch das verschiedene Transformationsverhalten bei verschiedenen Typen von Nominalisierungen und bei verschiedenen Typen von Adverbialkonstruktionen nicht erklärt werden kann" (Bartsch, 1972, 9).

Ich habe die Auseinandersetzungen um das Buch von Bartsch und seine Anwendung als Beschreibungsmodell für objekte der natürlichen Sprache - hier für die Adverbiale - referiert, weil ich, geleitet von linguistischem Interesse, damit zum Ausdruck bringen möchte, daß $i c h$ in diesem Beschreibungsmodell eine Hypothese sehe - und zwar eine brauchbare - um die weiterhin offenen Fragen $z u$ den Funktionsweisen der Adverbiale ${ }^{15}$, einschließlich der Berücksichtigung ihrer verschiedenen Klassen $z u$ behandeln. Ich möchte diese Arbeit nicht so verstanden wissen, $\mathrm{da} \beta$ eine Bestätigung der Analysen von Bartsch, angewendet auf das Russische, auch eine Bestätigung für die gemäß Bartsch anzusetzende intensionale Struktur logischer Entitäten sei. ${ }^{16}$ Mir scheint, daß im Hinblick auf folgende zwei Punkte, auf die mit unterschiedlichen Problemstellungen auch die Beispiele (1-19) hinweisen, die Ergebnisse von Bartsch für eine linguistische Darstellung der Adverbiale weiterführende Klassifikationskriterien geliefert haben:

1. Für die Rückführung der adverbialen nominalisierten Phrase auf einen zugrundeliegenden Satz, im besonderen dabei für die 
Bestimmung der Beziehung der übrigen Satzglieder zu dem ermittelten Prädikat;

2. für die Beschreibung der satzsemantischen Funktionen der Adverbiale durch die Einführung von Prädikatsvariablen.

Zur Illustration dieser Ergebnisse mögen folgende Ausführungen $z u$ Bartsch dienen:

Die Kritik von Bartsch an Steinitz (s. Adverbialsemantik, Kap. XIX) setzt an den Punkten an, die zeigen, daß die Versuche einer Analyse von PP mit Hilfe des Chomsky'schen Modells der strikten Subkategorisierung zu keiner eindeutigen Klassifizierung der Adverbiale führen. Bartsch erhebt zugleich den Anspruch, ein Modell vorzulegen, das darstellt, wie die semantische Ebene der Sprache und deren Verhältnis zur syntaktischen bestimmt werden sollen.

Es handelt sich um ein satzsemantisches Modell.

"Dabei werden syntaktische Beziehungen in ihrer Funktion durch allgemeine semantische Beziehungen mittels einer erweiterten Prädikatenlogik dargestellt" (3). Auch dieses Modell umfast einen Transformationsteil. Mit Hilfe einer "Testbatterie" (22) werden die syntaktischen Relationen zwischen den Konstituenten von Sätzen mit Adverbialkonstruktionen untersucht. Jedoch ist das Modell von Satzstrukturen, präziser von Aussagen, insoweit verändert, als es durch die logisch-semantische Ebene erweitert ist und von dieser bedingt wird.

Es ist ein Anliegen von Bartsch zu zeigen - und darin sieht sie die weitergehende Generalisierung durch ihr Modell -, daß da, wo die Phrasenstruktur-Marker keine eindeutige Antwort auf die abzuleitenden Sätze geben, diese Mehrdeutigkeiten auf der Ebene der logischen Satzsemantik aufgelöst werden können.

Zur Illustration des Analyseverfahres bei Bartsch seien die folgenden einleitenden Ausführungen $z u$ dem Unterschied von Satzadverbialen $z u$ anderen Adverbialen kurz referiert. 
In der Auseinandersetzung mit Darstellungen zur Nominalisierung bei Harris und anderen ( $14 \mathrm{f}$ ) wird gezeigt, daß bestimmte Adverbialkonstruktionen durch logisch-semantische Verhältnisse bedingt sind. So ist z.B.

"(d) Sein Lesen ist erfreulich." (15) doppeldeutig.

Es kann sein:

"(d') Daß er liest, ist erfreulich. Er liest erfreulicherweise.

und

"(d'') Wie er liest, ist erfreulich. Er liest erfreulich." (Ibid.).

Dagegen ist "Sein Französisch-Sprechen ist fließend", bzw. "Er spricht fließend Französisch", eindeutig. Es kann nur bedeuten: "Wie er Französisch spricht, ist fließend." Die Möglichkeit bzw. Blockierung der Modifizierung einer Aussage wie "x liest", "x spricht" in diesem Fall durch Adverbialkonstruktionen vom Typ eines Satzadverbials (wie in d') erklärt Bartsch durch bestimmte, unterschiedliche logischsemantische Relationen zwischen Prädikat und Adverb, die hier relevant sind: mit dem Satzadverbial in (d') wird "etwas über einen Sachverhalt - insbesondere über eine Tatsache nämlich darüber, daß etwas der Fall ist - ausgesagt" (16). Dagegen wird in den Sätzen "(d'') wie er liest, ist erfreulich", und "Er spricht fließend Französisch" etwas "über einen Handlungsvollzug oder -ablauf (Vorgang) ausgesagt" (16); dies ist die einzige Bedeutung von "Er spricht fließend Französisch"; denn es "kann nicht die -Tatsache, daß jemand spricht, wohl aber der Sprechvorgang 'fließend' genannt werden" (17).

Mit dieser zurückführung der semantischen und strukturellen Homonymie von z.B. (d) auf die logischen Individuenvariablen "Sachverhalt/Tatsache" einerseits und "Handlungsvollzug, ablauf, Vorgang" andererseits könnte, so meine ich, wenn diese sich als relevant für die Erweiterung von Sätzen durch Adverbiale erweisen, eine Vereinfachung der semantischen Merkmale erreicht werden ( $s$. dazu auch Bemerkungen von 
Bartsch zu Greenbaum (17)). Ich übernehme als heuristisches Mittel für die Klassifizierung der Adverbiale die von Bartsch angewandte Unterscheidung folgender Individuenvariablen:

1. Vorgangs- und Zustandsvariable,

2. Ereignis- und Umstandsvariable,

3. Sachverhalts- und Tatsachenvariable (77).

Ich komme nun $z u$ dem anderen der oben genannten $z u$ berücksichtigenden Lösungsvorschläge, nämlich $z$ u dem Problem der Rückführung adverbialer nominalisierter Phrasen auf zugrundeliegende Sätze. Fragen, die in der linguistischen Literatur mit den Begriffen "Nominalkonstruktion, Deprädikation, Kondensation" (Panevová, 1978a, 90) verbunden sind, werden ebenfalls in der logischen Semantik bei der Bedeutungsbestimmung von Sätzen diskutiert. Die Analyse von Sätzen wie:

(a) Im Bett sieht Hans den Abendstern.

(b) Im Westen sieht Hans den Abendstern.

gehört $z u$ den klassischen Aufgaben der analytischen Philosophie.

Bartsch führt diese Sätze in zusammenhang mit der Bedeutungsanalyse der lokalen Adverbiale an (125 f), sie ist jedoch bei anderen Adverbialen entsprechend anzuwenden. Die Analyse von (a) und (b) sieht danach folgendermaßen aus:

(a') Hans sieht den Abendstern. Gleichzeitig ist Hans im Bett.

(b') Hans sieht den Abendstern. Gleichzeitig ist der Abendstern im Westen.

Abgesehen davon, daß diese Bedeutungsanalyse die sprachliche struktur nur grob erfaßt, kann eine linguistische Beschreibung für die Funktionsweisen der freien Adverbiale doch einen Gewinn aus der vereinfacht illustrierten sprachanalytischen Analyse ziehen, und zwar durch Anwendung des praktizierten Prinzips der Auflösung homonymer 
Strukturen, das darin besteht, daß das Adverbial von verschiedenen Individuenvariablen prädizierbar ist; diese können objektsprachliche NP oder VP sein. Die Ableitung von freien Adverbialen aus Sätzen geht, wie dargestellt, auf den $\phi_{c}$ - Operator bei Harris zurück. Unklar blieb bei der $\phi_{c}-$ Operation, wie bereits ausgeführt, wie die damit explizit werdenden Beziehungen der Satzglieder zueinander beschrieben und in Regeln dargestellt werden können. Ubernimmt man im Prinzip die vorgeschlagene Auflösung homonymer Satzstrukturen, so ergeben sich für eine linguistische Analyse zwei Fragenkomplexe:

1. Auf welches Satzglied des Ausgangssatzes bezieht sich das durch die Rückführung des Adverbials auf einen Satz ermittelte Prädikat?

2u dieser Frage gibt es zwei mögliche Antworten:

Entweder lassen die allgemein semantischen Verhältnisse nur eine Möglichkeit der Beziehung zu (so bei (a)), oder der Satz ist ambig, wie z.B. (c):

(C) Hans sieht den Ball auf der Straße.

Dieser Satz kann bedeuten:

(c') Hans sieht den Ball. Gleichzeitig ist Hans auf der Straße.

(c'') Hans sieht den Ball. Gleichzeitig ist der Ball auf der Straße.

Der 2. Fragekomplex betrifft die wahl des Satzkonnektors, der bei den Sätzen (a) - (c) nach Bartsch das temporale "gleichzeitig" (125) ist. Gegen den Vorschlag von Steinitz, freie Lokaladverbiale auf einen temporalen NS zurückzuführen, führt Bartsch an, daß die Bedeutung von (a) auch einen Satz mit folgender Oberflächensyntax zuläßt: 
(a'') Der im Bett sich befindende Hans sieht den Abendstern.

Ohne den Untersuchungen an empirischem Sprachmaterial vorzugreifen (s. z.B. Kap. 3.1.2) scheint mir, daß das Problem des Verhältnisses von "kondensiertem" Adverbial $\mathbf{z u}$ syntaktischen Oberflächenstrukturen in den folgenden Ausführungen von Bartsch - hier zu den Adverbialen des Grundes zutreffend illustriert wird:

"Adverbiale Bestimmungen des Grundes oder des Zwecks enthalten zumeist das zweite Argument der Relation in so stark verkürzter Form, daB sie nur in einem Kontext oder situationsabhängig in eindeutiger weise interpretierbar sind. Aus dem Kontext oder der Situation mu bei ihrer Verwendung hervorgehen, welcher Satz der adverbialen Bestimmung zugrundeliegt: Die Verwendung dieser und anderer relationaler Adverbial-Konstruktionen setzt - falls sie das zweite Argument der Relation in stark verkürzter Form enthalten bei Sprecher und Hörer die gemeinsame Kenntnis bestimmter Informationen voraus.

z.B. "Peter kommt wegen des Staubsaugers".

"Peter kommt, weil er den Staubsauger reparieren will."

oder "Peter kommt, weil er den Staubsauger ausborgen will." oder "Peter kommt, weil er den Staubsauger verkaufen will."

Wegen der Verkürzung und der Vermeidung unnützer Redundanz ist die Verwendung relationaler Adverbiale günstig in allen situationen, in denen die relevanten Informationen bei Sprecher und Hörer gemeinsam vorauszusetzen sind - sei es durch im Text vorweg gegebene Informationen oder durch einen bei Sprecher und Hörer 19 ziemlich homogenen Informations- und Interessenstand" (110).

Diese Ausführungen treffen im besonderen auf Sätze zu, in denen durch das Adverbial abstrakte Relationen (kausal, final u.a.) ausgedrückt werden. Während bei lokalen, temporalen und modalen Adverbialen die durch diese ausgedrückten Relationen 
ihre semantischen Entsprechungen in der lexikalischen Bedeutung des Nomens der NP haben (vgl. "im Zimmer", "am Abend", "in Eile") ist das bei den abstrakten Relationen in der Regel nicht der Fall. Diese sprachlichen Gegebenheiten, die die von Bartsch diskutierte Mehrdeutigkeit motivieren, führten zu der Begrenzung der vorliegenden Untersuchungen auf lokale, temporale und modale Adverbiale. 
2.1.5 DIE "TIEFENSTRUKTUR" IN NEUEREN ARBEITEN R. RŮZ̆IČKAS UND SEINES KREISES

Zu Beginn dieser Einleitung stellte ich zwei Behauptungen auf :

1. daß eine linguistische Darstellung der Advb im Rahmen eines Satzverknüpfungsmodells erfolgen müsse:

2. daß dieses Modell die Relation von "Form" und "Inhalt" positiv beschreiben müsse.

In der bisher dargelegten Beweisführung $\mathrm{zu}$ diesen Behauptungen wurden folgende Ergebnisse gewonnen, die sich aus Untersuchungen, durchgeführt auf der Basis von Sprachbeschreibungsmodellen (2.1.1 - 2.1.4), ableiten lieben:

1. Advb gehen auf prädikative Ausdrücke zurück;

2. Advb gehören nicht zum Verbnucleus, sie sind auf Sätze zurückzuführen;

3. Advb werden auf der Ebene der satzsemantischen Relationen als Funktoren repräsentiert;

4. Advb drücken inhaltliche Relationen aus, die als unterschiedliche Prädikationen über bestimmte Klassen von Argumenten darstellbar sind.

Die neuesten Arbeiten von Růzicka und seines Kreises scheinen mir fur die Argumentation über ein Sprachbeschreibungsmodell, das die Relation von "Form" und "Inhalt" einbezieht, eine Lücke zu schließen, die es erlaubt, die Ergebnisse oben 1-3 und 4 zueinander in Beziehung zu setzen.

Im Zentrum der Modellierungsvorschläge steht eine Neubestimmung des Begriffs "Tiefenstruktur". Gegen die Positionen der generativen Semantiker führt Růzicka aus, daß diese vor dem Problem standen, "im homogenen Durchgang einer Derivation die schwer übersehbare Distanz zwischen Bedeutungen und syntaktischen Oberflächenstrukturen $z u$ überwinden. Diese ist unwegsam, wenn die Zwischenstation einer syntaktischen Tiefenstruktur zwischen den zwei unabhängig 
spezifizierbaren Bereichen Bedeutung und Laut aufgegeben ist. Daß ihre wechselseitige zuordnung im Modell einer homogenen Vereinigung von Syntax und Semantik theoretisch und empirisch bewältigt werden kann, ist nicht zu erwarten. Es ist zweifelhaft, ob die Syntax ein ausreichendes methodologisches Paradigma für Semantik ist" (Rủzička, 1980, 20).

Das neue Modell für die Beziehungen der beiden Bereiche "Bedeutung" und "Laut", die sich zum einen als logisches Prädikat mit seinen Argumenten, zum anderen als Lexem mit seinen Leerstellen spezifizieren lassen, bestimmt die Tiefenstruktur als die Beschreibungsebene, für die gilt, daß sie "durch die Lexeme maximal direkt mit der Darstellungsebene der semantischen Struktur in Relation stehen. Sie ist zugleich eine art lexikalisch-syntaktischer Invariante, ein abstrakter Repräsentant der Vielzahl syntaktisch synonymer und durch die syntaktische Paradigmatik verbundener Sätze. Die Darstellung des Satzes auf der lexikalischsyntaktischen tiefenstrukturellen Ebene kann man als Ausgangskonstruktion betrachten. Die syntaktisch umgeformten Konstruktionen können dann als abgeleitet gelten" (zimmermann, 1978, 72).

Rủziłka sieht die Gefahren, die mit der Annahme dieser "maximal direkten Entsprechung" verbunden sind: "Die Versuche, eine Tiefenstruktur zu postulieren, laufen Gefahr, sich $z u$ einem zirkel des syntaktischen Konstrukts zu schließen, in dem Kriterien der innersyntaktischen Motiviertheit und Kriterien der semantischen Durchsichtigkeit der syntaktischen Struktur einander tautologisch stützen" (Růziðka, 1980, 14). Die Gefahr von zirkelschlüssen bei der Bestimmung der Ausgangskonstruktion einerseits und der abgeleiteten Konstruktionen andererseits wird, soweit mir bekannt, durch Untersuchungen $z \mathrm{u}$ den Lexemen unterlaufen; z.B. wird mit der Diathese des verbalen Lexems dessen zughehörigkeit zu einer Prädikatsklasse bestimmt. Aufgrund 
dieser Zuordnung läßt sich die Ausgangskonstruktion gewinnen, bei der die Hierarchie der syntaktischen Leerstellen der Hierarchie der semantischen Argumente entspricht; das Lexem wird hier in seiner "Ausgangsdiathese" (Zimmermann, 1978, 72) verwendet. In den abgeleiteten Konstruktionen liegt dagegen Hierarchiedivergenz (z.B. in passiven Sätzen) vor. Mit diesem Beschreibungszusammenhang kann die verwendung der PP ${ }_{\text {loc }}$ in den Satzpaaren oben (10) - (11) / (12) - (13) systematische eingeordnet, und die Satzpaare können als syntaktische Synonyme bestimmt werden.

Außer für unser spezielles Problem (s. dazu unten Kap. 3.1.3, bes. 79) scheint die von Rủžiðka vorgenommene Neubestimnung des Begriffs "Tiefenstruktur" auch Lösungsmöglichkeiten für die Fragen zu geben, die im Standardmodell mit dem Satzpaar "(19) (i) John strikes me as pompous - I regard John as pompous" (204, s. auch Anm. 13, S. 284 f) zur Sprache kommen. Aufgrund des Standardmodells, das nur solche Sätze als semantisch invariant bestimmt, für die sich - eventuell durch Transformationen - syntaktische synonyme strukturen nachweisen lassen, kann Chomsky das Satzpaar (19) (i) nur als "annähernde Paraphrase" bezeichnen und auf das damit für eine Grammatikbeschreibung aufgeworfene Problem naher "Bedeutungsbeziehungen" verweisen. Nach der Konzeption der Tiefenstruktur von Rủzicka dürfte die Lösung darin zu suchen sein, daß die Lexeme "strike" und "regard" Prädikate verschiedener Klassen im Hinblick auf ihre syntaktischen Leerstellen und semantischen Argumente wie auch deren Hierarchie sind. Dann wird plausibel, daß in dem Satzpaar (19)(1) oberflächenstrukturell ähnliche Sätze vorliegen, die aber in der Ableitungshierarchie zu ihren Ausgangskonstruktionen auf je verschiedenen Stufen einzuordnen sind. 
2.2 THEORETISCHE UND METHODISCHE SCHLUßFOLGERUNGEN

Das Studium des empirischen Materials von ca. 3000 Belegen präpositionaler adverbialer Phrasen (PP) aus belletristischen und publizistischen Texten hat unter Berücksichtigung der oben diskutierten linguistischen Modelle zu der Konzeption des folgenden Beschreibungsansatzes geführt:

Als allgemeiner Rahmen dient die FGB (Prag).

Die für die Darstellung von Advb primär relevanten Ebenen sind die satzsemantische Ebene und die Satzgliedebene. Für die Beschreibung der Satzgliedebene verwende ich die Regeln des Standardmodells, die der satzsemantischen Relationen erfolgt in Anlehnung an die "Adverbialsemantik".

Die zuordnung von satzsemantischer und Satzgliedebene wird mit Hilfe der "lexikalisch-syntaktischen Tiefenstruktur" ( $R$. Růzicka) modelliert, die als "lexikalisch-syntaktische Invariante" oder "abstrakter Repräsentant" syntaktisch synonymer sätze definiert ist.

Dieses als Hypothese formulierte Sprachbeschreibungsmodell wird durch Tests an der objektsprache überprüft oder vice versa: die Objektsprache wir mit Hilfe der Tests durch das Modell klassifiziert. Die rests sind als operative Kriterien zur Bestimmung der Funktionen der Advb im Satz und der Ermittlung sprachlicher Synonymien und Homonymien konzipiert, sie haben metasprachliche Funktion. Ein weiteres Ergebnis der Tests ist die Feststellung syntaktisch synonymer loberflächen)strukturen, die durch Transformation aufeinander beziehbar sind.

Für die Beschreibung der drei "Primärklassen" von Advb in der Form von PP - nämlich lokal, temporal und modal - erwies es sich als notwendig, 5 Sorten von Tests anzuwenden; es sind dies: 1. Prädikations-Tests, 2. Dialog-Tests, 3. NegationsTest, 4. Prädikatsvariablen-Test, 5. Thema-Rhema-GliederungsTest. Dabei stellte sich heraus, das die Explikationskraft 
von 1 und 2 gleichgewichtig für die 3 untersuchten Klassen von $A d v b$ ist, daB sie jedoch bei den Tests 3-5 unterschiedlich ist, aber nicht widersprüchlich in dem Sinn, daß diese die Ergebnisse einer anderen Testsorte widerlegen würden. Es handelt sich vielmehr darum, daß z.B. der Negations-Tests ${ }^{20}$ für die Beschreibung der Modaladverbiale in anderer Weise relevant ist als für die lokalen und temporalen Advb, oder die Tests zur Thema-Rhema-Gliederung für die lokalen Advb relevant sind, während sie es für die temporalen und modalen kaum sind. Diese Unterschiede in der Explikationskraft der Tests für die verschiedenen PP ist durch die Funktion der Advb im Satz ${ }_{21}$ die bei den drei untersuchten Klassen deutlich erkennbare Unterschiede aufweist, bedingt. Aus praktischen Gründen werden daher in diesem einführenden Kapitel nur der Prädikations-Test und der Dialog-Test illustriert, dabei sind die gemeinsamen strukturellen Eigenschaften der Advb von Interesse; ihre Unterschiede werden in den entsprechenden Kapiteln der jeweiligen Adverbiale zur Sprache kommen. Die zu beobachtenden Gemeinsamkeiten grenzen die Advb von anderen Satzgliedklassen ab, insbesondere von denen der "obligatorischen" Adverbiale, die zum Verbnucleus gehören. Sie erlauben andererseits, die untersuchten Advb - ungeachtet der Unterschiede zwischen ihnen - als eine Klasse syntaktischer strukturen zu definieren.

\section{Der Prädikations-Test}

Der Prädikations-Test ist eine weiterentwicklung des Konstituentensatz-Tests, wie er auch aus Harris bekannt ist -

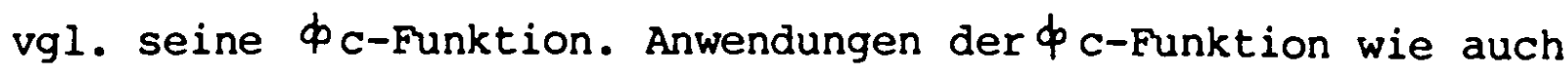
Chomsky's Ausarbeitungen $z u$ der Rekursivität sprachlicher Strukturen, als deren Ergebnis komplexe Sätze verstanden werden, lassen für eine Analyse von Advb zwei zentrale Fragen offen: 
1. Welche Struktur hat der angenommene zugrundeliegende Satz? 2. Welche Beziehungen hat er zu dem übrigen Satz? zu diesen 2 Fragenkomplexen versucht der Prädikations-Test Lösungen $z u$ geben. Die Untersuchung erfolgt in folgenden Schritten:

- das Adverbial in der Form von PP wird daraufhin überprüft, ob es auf eine $S_{T S}$ zurückgeführt werden kann. Diese erste "grobe" Analyse ergibt 2 Klassen von PP: - PP, die auf einen $S_{T S}$ zurückgeführt werden können $\left(P^{+S} T S\right.$ ) und

- PP, die sich nicht auf einen $S_{T S}\left(P P^{-S} T S\right.$ ) zurückführen lassen.

Die weitere Analyse der Klasse $\mathrm{PP}^{-\mathrm{S}}$ TS wird hier nicht verfolgt - positive Ergebnisse zu dieser Klasse leistet ein anderer Test, der Dialog-Test. Die weitere Analyse für die Klasse PP TS versucht aufzuklären, welche Beziehungen zwischen der PP und den übrigen Satzgliedern vorliegen.

Zu diesem Zweck werden folgende Fragen gestellt:

1. Welches Glied (welche Glieder) des Satzes wird (werden) durch das 22 aus der PP erschlossene Prädikat des $S_{\text {.TS }}$ näher bestimmt.

Diese Fragestellung ist aus prädikatenlogischen Arbeiten übernommen (s. oben 2.1.4). Der Test ist jedoch so konzipiert, daß er auf die Untersuchung linguistisch relevanter Strukturen, wie syntaktische synonymie und Homonymie; abzielt. Die Analyseschritte sehen schematisch folgendermaßen aus:
(I) $1 . \mathrm{s}_{\mathrm{OF}}$
$\mathrm{NP}^{\mathrm{l}}$
$\operatorname{VP}^{1}\left(N P^{n-1}\right)$
PP
( für
PP

$\begin{array}{llll}\text { 2. } & \mathrm{S}_{\mathrm{TS}} \longrightarrow \\ \text { 3. } & \mathrm{VP}^{2} \longrightarrow \mathrm{NP}^{\mathrm{x}} & & \mathrm{VP}^{2} \\ \text { 4. } \mathrm{PP} & \longrightarrow \mathrm{Vb}^{2} & \mathrm{PP} & \\ \text { Adverb } & & \end{array}$


Zur Illustration des Prädikations-Tests bieten sich Beispielsätze an wie:

$z u$ I: vor isčez $\left\{\begin{array}{l}\mathrm{v} \text { tolpe ljudej / tam. } \\ \mathrm{v} \text { polnoc'/ togda. } \\ \text { nezametno. }\end{array}\right\}$

$z u$ 2: Vor iscez. Ėto bylo $\left\{\begin{array}{l}v \text { tolpe ljudej / tam. } \\ v \text { polnot } / \text { togda. } \\ \text { nezametno. }\end{array}\right\}$

2. Von welcher Art ist die Prädikation? Lokal? Temporal? Modal? Mit dieser Frage, die auf klassenkonstituierende Merkmale der Adverbiale abhebt - s. dazu Kap. 3 - 5 - stellt sich das Problem, daß wir Klassen festlegen, die der Kapiteleinteilung der "Adverbialbestimmungen" in traditionellen Grammatiken $z u$ entsprechen scheinen. Es kann $z u$ Recht der Einwand erhoben werden, daß diese traditionellen, nach inhaltlichen Kriterien gewonnenen "Kapitel" der Grammatik Fremdkörper innerhalb des hier vorgestellten Beschreibungsmodells sind. Dieser vom theoretischen Standpunkt begründete Einwand könnte durch einen praktischen gestützt werden, denn die Einordnung in lokale, temporale etc. Adverbiale der mit formalen Mitteln gewonnen Klassen $\mathrm{PP}{ }^{+S}$ TS und $\mathrm{PP}^{-S}$ TS wird fragwürdig: inhaltliche Angaben, z.B. "wo" etwas ist, geben auch Sätze mit PP ${ }^{-S}$ TS. Die Argumentation gerät in diese widersprüche, solange die Frage nach der Art der Prädikation oberflächengebunden und ohne zusammenhang mit der Frage 1, nämlich ob in der sprachlichen Strukturierung eine Prädikation vorliegt, d.h. die PP zu der Klasse PP TS gehört, verstanden wird. Frage 2 zielt auf eine satzsemantische Analyse, d.h. eine Analyse der Relation zwischen Prädikator und dessen möglichen Argumenten.

Das zu beobachtende Problem, daß die Differenzierungseinheiten - hier die traditionellen - von einem formalen Analysestandpunkt aus nicht-disjunkte Klassen ergeben - 
scheint mir ein allgemeines zu sein: Es würde in ähnlicher weise wieder auftreten, wenn ich die Differenzierungseinheiten aussschließlich vom Standpunkt der syntaktischen oder ausschließlcih vom dem - nun präzisiert - der satzsemantischen Analyse ableiten würde. Dieser Umstand scheint mir zum einen von der gestellten Aufgabe, zum anderen durch das Untersuchungsobjekt bedingt $z u$ sein: Es sollen Formen und Funktionen der Advb beschrieben und daraus Regeln für die Erweiterung von Ausdrücken mit Advb formuliert werden. Ich bediene mich dabei eines Modells, das aus einem umfassenderen Sprachbeschreibungsmodell mit mehreren, untereinander geordneten Ebenen für die Beschreibung sprachlicher Einheiten abgeleitet ist (FGB). Eine jede Ebene und die Beziehungen der Ebenen zueinander - und damit komme ich zu den spezifischen Eigenschaften des Untersuchungsobjekts sind durch den asymmetrischen Dualismus von Form und Funktion des sprachlichen zeichens überhaupt bedingt. Aus diesen Zusammenhängen folgt, daß für eine Beschreibung mit ausschließlich disjunkten Klassen sich die Darstellung von Sprache auf eine bestimmte Ebene beschränken müßte. Für eine Lösung der hier gestellten Aufgabe ist aber die Analyse von Advb auf mehreren Ebenen - der satzsemantischen und Satzgliedebene - unumgänglich. Die Diskussion zu den vorliegenden Arbeiten über Advb (2.1.1, 2.1.2) zeigen deutlich, das eine Analyse, die sich auf die syntaktische Ebene, d.h. die formalen Mittel beschränken würde, unbefriedigend ausfällt. Die Auflösung der Mehrdeutigkeiten der syntaktischen Struktur von Sätzen mit Advb erfolgt durch die Einbeziehung der Bedeutungsstruktur von Sätzen (der tektogrammatischen Ebene nach der FGB) in die Beschreibung diesem Analyseschritt dienen zusammenhängend die Fragen: über welches Satzglied wird prädiziert? und - Von welcher Art ist die Prädikation?

Zweifellos sind mehrere Möglichkeiten für einander korrigierende und ergänzende Untersuchungen $z u$ "Form" und "Inhalt" denkbar. 
Ich habe mich an dieser stelle dafür entschieden, das traditionelle, von Grammatik und Logik verwendete Einteilungsschema der Adverbiale zu übernehmen und innerhalb dessen aufgrund der Berücksichtigung der Analysen der verschiedenen Ebenen disjunkte Klassen abzuleiten.

I. 4 (oben) kann damit ersetzt werden:

I.5 Advb $\longrightarrow\left\{\begin{array}{l}\text { Lok } \\ \text { Temp } \\ \text { Mod }\end{array}\right\}$

Ich komme zu einer letzten Frage, die sich im Rahmen des Prädikations-Tests stellt:

- Von welcher Art ist der Satzkonnektor?

Die Beantwortung dieser Frage hängt von der Beantwortung der vorausgehenden Fragen 1 und 2 ab. $\mathrm{zu}$ ihrer vorläufigen Erläuterung soll hier nur erwähnt werden, daß sie die Art der Verknüpfung von $S{ }_{\text {TS }}$ und $S^{2}$ TS untersucht (dazu $S .71 \mathrm{ff}$ ) und der Disambiguierung von Advb dient, wenn diese nach der satzsemantischen Analyse mehreren Klassen zugerechnet werden können (s. dazu u. 4.4.2).

\section{Der Dialog-Test}

Der Prädikations-Test ergibt für die Klasse von PP, die sich nicht auf einen $S_{T S}$ zurückführen lassen, ein negatives Resultat: ${ }^{-S^{-S}}$ TS. Diese PP lassen sich - wie umfangreiche empirische Untersuchungen (Panevová (1978b), Beranová (1975)) ergeben haben, deren Ergebnisse ich hier übernehme - durch den Dialog-Test positiv beschreiben. Wie oben ( $\mathrm{S} .30 \mathrm{ff}$ ) referiert, handelt es sich bei den $\mathrm{PP}^{-\mathrm{S}}$ TS um die obligatorischen Adverbialen, Aktanten des Verbs, für die sich der Dialog-Test als geeignetes Beschreibungskriterium erweist (s.u. Typ 2). 
Der Dialog-Test gibt andererseits keine positiven Aussagen über Sätze mit PP ${ }^{+S}$ TS (s.u. dazu Typ 1). Prädikations-Test und Dialog-Test sind damit einander ergänzende Kriterien einerseits für die Unterscheidung von obligatorischen und freien Adverbialergänzungen und andererseits für die Bestimmung - positiv oder negativ - ihrer strukturellen Merkmale. Unter diesem Gesichtspunkt - als Uberprüfungskriterium zur Abgrenzung der freien adverbialen Ergänzungen des Verbs von denen zum Verbnucleus gehörenden adverbialen Aktanten - wird der Dialog-Test in dieser Arbeit verwendet.

Typ 1:

- vor isčez.

$$
\left\{\begin{array}{l}
\text { Gde } \\
\text { Kuda } \\
\text { Kogda } \\
\text { Kak } \\
\text { etc. }
\end{array}\right\} \text { istez vor? }
$$

- Ne znaju.

- v tolpe ljudej. / V polnot'. / Lovko.

Typ 2:

- ... i svidetel' dolgo ešce stojal. - Gde?

- Von na meste prestuplenija.

- Ne znaju. 


\section{LOKALE ADVERBIALE}

Die Beobachtungen an Sätzen mit freien Lokaladverbialen (im ff Advb loc' - hier in der Form von PP - lassen unschwer erkennen, daß deren Funktionen im Satz mannigfaltig und nicht selten mehrdeutig sind. Man vergleiche die folgenden Beispiele, von denen (1), (4), (8) der Belegsammlung entstammen; die übrigen sind von mir unter Beibehaltung der Lexik und Morphologie, jedoch mit wechsel in der Wortfolge konstruiert. (Es werden nur die für unsere Fragestellung wichtigen Wortfolgemöglichkeiten angeführt.)

(1) Vor isčez v tolpe ljudej.

( 2) V tolpe ljudej istez vor.

( 3) Vor v tolpe ljudej isčez.

( 4 ) Pionery videli pervogo sekretarja obkoma na stroitel'noj plošcadke.

(5) Na stroitel'noj ploscadke pionery videli pervogo sekretarja obkoma.

( 6) Pionery na stroitel'noj plošadke videli pervogo sekretarja obkoma.

( 7) Pionery videli na stroitel'noj plošcadke pervogo sekretarja obkoma.

( 8 ) Ne konCaetsja trudovoj den' na prostorach strany.

(9) Na prostorach strany ne konXaetsja trudovoj den'. (10) Trudovoj den' na prostorach strany ne konČaetsja.

Bei identischer Lexik und Morphologie, jedoch bei wechselnder Wortfolge bedeuten die Sätze eines jeden Beispielsets (1-3), (4-7), (8-10) Verschiedenes bzw. können etwas Verschiedenes bedeuten. Als relevant für die Realisierung verschiedener Bedeutungen durch Advb ${ }_{\text {loc }}$ lassen sich in einfachen Sätzen feststellen:

Stellung am Satzende, Stellung am Satzanfang und Stellung der Lokaladverbiale vor und/oder nach dem finiten Prädikat. Die 
Darstellung der syntaktischen und satzsemantsichen Regeln hierfür erfolgt in zwei Arbeitsschritten:

Zunächst werden sätze mit einem ${ }^{A d v b}{ }_{10 c}$ in einer bestimmten Position, nämlich in stellung am Satzende, untersucht ${ }^{2}$. Die Sätze repräsentieren verschiedene Satz- und Aussagestrukturen - gemeinsam ist ihnen die Erweiterung durch Advb ${ }_{\text {loç Das }}$ Untersuchungsverfahren ist generativ und transformationell: Es werden mit Hilfe des Prädikations-Tests Regeln abgeleitet, nach denen modellhaft die Erweiterung eines Satzes durch Advb ${ }_{\text {loc }}$ verläuft oder - anders gesagt - ausgehend von Sätzen

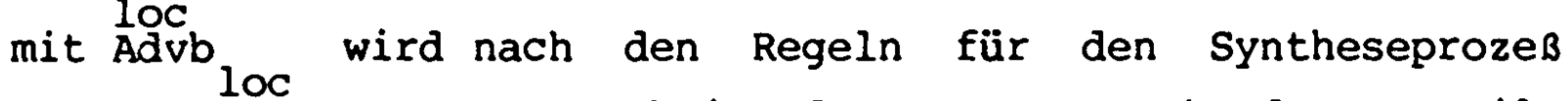
gefragt, als dessen Ergebnis der zu untersuchende Satz gilt (Kap. 3.1). Es wird damit auch die Hypothese von den freien Adverbialen als deprädikativer Aussageform im Satz überprüft und der Versuch gemacht, diese bisherigen Ergebnisse zu präzisieren. Als Nebenergebnisse kommen dabei auch Beziehungen zu anderen - strukturell synonymen - Oberflächenstrukturen zur Sprache.

Der zweite Arbeitsschritt berücksichtigt den Oberflächensatz. Er untersucht die Folgen und fragt nach den Regeln, die beim Stellungswechsel des Lokaladverbials im Satz zu beobachten sind (Kap. 3.2). Die Untersuchung erforscht den funktionalen Aspekt des Satzes. Sie behandelt den Fragenkomplex im Rahmen der Thema-Rhema-Gliederung.

Die beiden Untersuchungsprozeduren zur Bestimmung der Funktionen von Advb ${ }_{\text {loc }}$ gehören zusammen. Sie werden hier aus methodischen Uberlegungen getrennt und in der angegebenen Reihenfolge angewendet. Die Analyse ist auf expressiv neutrale Beispielsätze mit "normaler" Intonation beschränkt. Die Erweiterung des generativ-transformationellen Ansatzes um den der Topic-Comment-Gliederung erweist sich als notwendig, wenn man eine möglichst generelle Beschreibung für die Ableitung der Adverbiale, im besonderen der lokalen', anstrebt, wenn man also für die Analyse der Advb ${ }_{10 c} z \cdot B$. in 

den Sätzen (1)-(10) ungeachtet ihrer unterschiedlichen Bedeu-
tungen eine Grundfunktion meint nachweisen zu können. Die
Unterschiede ergeben sich durch Veränderung der Gliederung
der Aussage und führen - vom Standpunkt des Oberflächen-
satzes - zu unterschiedlichen Funktionen der Advb loc im
Text. Bei der Konzeption eines Modells zur Ableitung von
Sätzen mit Advb erhebt sich damit die Schwierigkeit,
einerseits die grammatischen Basisregeln für die Derivation
zu formulieren und andererseits für Aussagen einen
Erklärungszusammenhang aufzuweisen, der Sprachsystem und
Kontextabhängigkeit berücksichtigt. den Sätzen (1)-(10) ungeachtet ihrer unterschiedlichen Bedeu-
tungen eine Grundfunktion meint nachweisen zu können. Die
Unterschiede ergeben sich durch Veränderung der Gliederung
der Aussage und führen - vom Standpunkt des Oberflächen-
satzes - zu unterschiedlichen Funktionen der Advb loc im
Text. Bei der Konzeption eines Modells zur Ableitung von
Sätzen mit Advb erhebt sich damit die Schwierigkeit,
einerseits die grammatischen Basisregeln für die Derivation
zu formulieren und andererseits für Aussagen einen
Erklärungszusammenhang aufzuweisen, der Sprachsystem und
Kontextabhängigkeit berücksichtigt. den Sätzen (1)-(10) ungeachtet ihrer unterschiedlichen Bedeu-
tungen eine Grundfunktion meint nachweisen zu können. Die
Unterschiede ergeben sich durch Veränderung der Gliederung
der Aussage und führen - vom Standpunkt des Oberflächen-
satzes - zu unterschiedlichen Funktionen der Advb loc im
Text. Bei der Konzeption eines Modells zur Ableitung von
Sätzen mit Advb erhebt sich damit die Schwierigkeit,
einerseits die grammatischen Basisregeln für die Derivation
zu formulieren und andererseits für Aussagen einen
Erklärungszusammenhang aufzuweisen, der Sprachsystem und
Kontextabhängigkeit berücksichtigt. den Sätzen (1)-(10) ungeachtet ihrer unterschiedlichen Bedeu-
tungen eine Grundfunktion meint nachweisen zu können. Die
Unterschiede ergeben sich durch Veränderung der Gliederung
der Aussage und führen - vom Standpunkt des Oberflächen-
satzes - zu unterschiedlichen Funktionen der Advb loc im
Text. Bei der Konzeption eines Modells zur Ableitung von
Sätzen mit Advb erhebt sich damit die Schwierigkeit,
einerseits die grammatischen Basisregeln für die Derivation
zu formulieren und andererseits für Aussagen einen
Erklärungszusammenhang aufzuweisen, der Sprachsystem und
Kontextabhängigkeit berücksichtigt. den Sätzen (1)-(10) ungeachtet ihrer unterschiedlichen Bedeu-
tungen eine Grundfunktion meint nachweisen zu können. Die
Unterschiede ergeben sich durch Veränderung der Gliederung
der Aussage und führen - vom Standpunkt des Oberflächen-
satzes - zu unterschiedlichen Funktionen der Advb loc im
Text. Bei der Konzeption eines Modells zur Ableitung von
Sätzen mit Advb erhebt sich damit die Schwierigkeit,
einerseits die grammatischen Basisregeln für die Derivation
zu formulieren und andererseits für Aussagen einen
Erklärungszusammenhang aufzuweisen, der Sprachsystem und
Kontextabhängigkeit berücksichtigt. den Sätzen (1)-(10) ungeachtet ihrer unterschiedlichen Bedeu-
tungen eine Grundfunktion meint nachweisen zu können. Die
Unterschiede ergeben sich durch Veränderung der Gliederung
der Aussage und führen - vom Standpunkt des Oberflächen-
satzes - zu unterschiedlichen Funktionen der Advb loc im
Text. Bei der Konzeption eines Modells zur Ableitung von
Sätzen mit Advb erhebt sich damit die Schwierigkeit,
einerseits die grammatischen Basisregeln für die Derivation
zu formulieren und andererseits für Aussagen einen
Erklärungszusammenhang aufzuweisen, der Sprachsystem und
Kontextabhängigkeit berücksichtigt. den Sätzen (1)-(10) ungeachtet ihrer unterschiedlichen Bedeu-
tungen eine Grundfunktion meint nachweisen zu können. Die
Unterschiede ergeben sich durch Veränderung der Gliederung
der Aussage und führen - vom Standpunkt des Oberflächen-
satzes - zu unterschiedlichen Funktionen der Advb loc im
Text. Bei der Konzeption eines Modells zur Ableitung von
Sätzen mit Advb erhebt sich damit die Schwierigkeit,
einerseits die grammatischen Basisregeln für die Derivation
zu formulieren und andererseits für Aussagen einen
Erklärungszusammenhang aufzuweisen, der Sprachsystem und
Kontextabhängigkeit berücksichtigt. den Sätzen (1)-(10) ungeachtet ihrer unterschiedlichen Bedeu-
tungen eine Grundfunktion meint nachweisen zu können. Die
Unterschiede ergeben sich durch Veränderung der Gliederung
der Aussage und führen - vom Standpunkt des Oberflächen-
satzes - zu unterschiedlichen Funktionen der Advb loc im
Text. Bei der Konzeption eines Modells zur Ableitung von
Sätzen mit Advb erhebt sich damit die Schwierigkeit,
einerseits die grammatischen Basisregeln für die Derivation
zu formulieren und andererseits für Aussagen einen
Erklärungszusammenhang aufzuweisen, der Sprachsystem und
Kontextabhängigkeit berücksichtigt. den Sätzen (1)-(10) ungeachtet ihrer unterschiedlichen Bedeu-
tungen eine Grundfunktion meint nachweisen zu können. Die
Unterschiede ergeben sich durch Veränderung der Gliederung
der Aussage und führen - vom Standpunkt des Oberflächen-
satzes - zu unterschiedlichen Funktionen der Advb loc im
Text. Bei der Konzeption eines Modells zur Ableitung von
Sätzen mit Advb erhebt sich damit die Schwierigkeit,
einerseits die grammatischen Basisregeln für die Derivation
zu formulieren und andererseits für Aussagen einen
Erklärungszusammenhang aufzuweisen, der Sprachsystem und
Kontextabhängigkeit berücksichtigt. den Sätzen (1)-(10) ungeachtet ihrer unterschiedlichen Bedeu-
tungen eine Grundfunktion meint nachweisen zu können. Die
Unterschiede ergeben sich durch Veränderung der Gliederung
der Aussage und führen - vom Standpunkt des Oberflächen-
satzes - zu unterschiedlichen Funktionen der Advb loc im
Text. Bei der Konzeption eines Modells zur Ableitung von
Sätzen mit Advb erhebt sich damit die Schwierigkeit,
einerseits die grammatischen Basisregeln für die Derivation
zu formulieren und andererseits für Aussagen einen
Erklärungszusammenhang aufzuweisen, der Sprachsystem und
Kontextabhängigkeit berücksichtigt. den Sätzen (1)-(10) ungeachtet ihrer unterschiedlichen Bedeu-
tungen eine Grundfunktion meint nachweisen zu können. Die
Unterschiede ergeben sich durch Veränderung der Gliederung
der Aussage und führen - vom Standpunkt des Oberflächen-
satzes - zu unterschiedlichen Funktionen der Advb loc im
Text. Bei der Konzeption eines Modells zur Ableitung von
Sätzen mit Advb erhebt sich damit die Schwierigkeit,
einerseits die grammatischen Basisregeln für die Derivation
zu formulieren und andererseits für Aussagen einen
Erklärungszusammenhang aufzuweisen, der Sprachsystem und
Kontextabhängigkeit berücksichtigt.

zu formulieren und andererseits für Aussagen einen
Erklärungszusammenhang aufzuweisen, der Sprachsystem und
Kontextabhängigkeit berücksichtigt.

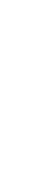


3.1 DAS LOKALADVERBIAL IN DER FUNKTION EINER PRÄDIKATION

3.1.1 DIE BEZIEHUNGEN ZWISCHEN SYNTAKTISCHER EBENE (VP, PP ${ }_{\text {lOC }}$ ' UND SEMANTISCHER EBENE ( ${ }^{1}{ }_{\text {TS }} \mathrm{S}^{2}{ }_{\mathrm{TS}}$ )

Die folgenden Sätze mit, Endstellung der PP reagieren auf Prädikations-, Negations- ${ }^{7}$ und Dialog-Test in der einleitend für freie Adverbiale dargestellten weise positiv, wie auch bei Substitution der PP durch andere Adverbiale; dagegen negativ bei bestimmten $\mathrm{Tf}_{8}$ in Zusammenhang mit dem Genus verbi ${ }^{8}$.

Man vergleiche:

(1) Vor iłcez $v$ tolpe ljudej.

Prädikations-Test

Negations-Test

Dialog-Test

Substitution von PP durch andere Adverbiale Tf Aktiv/Passiv
Vor isčez. On byl $v$ tolpe ljudej. Vor ne istez $v$ tolpe ljudej. Vor iskez ne $v$ tolpe ljudej. Vor iscez.

- Gde?/Kuda?

- Ne znaju.

Vor iš̌ez $\left\{\begin{array}{c}v \text { polnoč' } \\ \text { lovko. }\end{array}\right\}$

(11) Predstavitel'naja vstrexa pisatelej Azii i Afriki otkryvaetsja $v$ Taškente.

Bei Stellung der PP ${ }_{\text {loc }}$ am Satzanfang ist der PrädikationsTest für eine Analyse ungeeignet: 
(12) $V$ tolpe ljudej vor iscez.

Vor byl $v$ tolpe ljudej. Vor isčez.

Es wird unten Kap. 3.2.3 ausgeführt, daß die Gründe hierfür nicht darin $z u$ suchen sind, daß der Prädikations-Test nur begrenzt tauglich ist, sondern im zusammenhang mit der veränderten Position der PP ${ }_{\text {loc }}$ im Satz zu sehen sind.

Wie (1) verhalten sich bei Anwendung der Tests (ich beschränke mich auf die Anwendung des Prädikations-Tests) z.B.:

(13) Veselye i oživlennye gosti sumno razdevalis' $v$ perednej.

(14) Veselye i oživlennye gosti sumno razdevalis'. Oni byli $\checkmark$ perednej.

(15) Leonid Il'ił Brežnev vystupaet na sovešanii partijnochozjajstvennogo aktiva Kasachtana.

(16) Leonid Il'ił Brežnev vystupaet. On na sovešcanii partijno-chozjajstevennogo aktiva Kasachstana.

(17) Mal'tik spal $v$ dome storoža vodokałki.

(18) Mal'tik spal. On byl $v$ dome storoža vodokacki.

(19) Pervye zvezdy zazigalis' na rozovom nebe.

(20) Pervye zvezdy zažigalis'. Oni byli na rozovom nebe.

(21) Liza postriglas' v B...m monastyre.

(22) Liza postriglas'. Ona byla v B...m monastyre.

Bei (1), (13)-(22) handelt es sich um Beispielsätze der einfachen Satzstruktur:

$$
\mathrm{s}_{\mathrm{OF}} \longrightarrow \stackrel{\mathrm{NP}}{\left\{\begin{array}{l}
n \\
\varnothing
\end{array}\right.} \quad \mathrm{VP} \quad\left(\mathrm{PP}_{10 \mathrm{C}}\right)^{\prime}
$$

In einem ersten Versuch der Darstellung ihrer expliziten Bedeutung lassen sie sich als Transformation von in Beziehung gesetzten $S$ IS und $S^{2}$ TS darstellen, wobei die Art der Beziehung, in der $s_{\text {TS }}{ }_{\text {TS }} s_{\text {TC }}^{2}$ zueinander stehen, noch 
geklärt werden muß.

Für $s^{1}$ TS und $s^{2}$ TS nehmen wir folgende Ersetzungsregeln an ( I ):

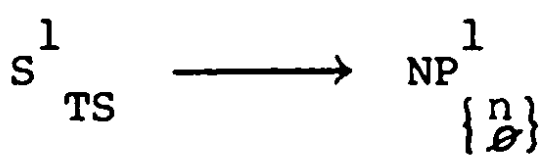

VP

$\mathrm{S}_{\mathrm{TS}}^{2} \longrightarrow \mathrm{NP}^{1 / \mathrm{S}^{1}} \quad$ byt $\quad \mathrm{PP}_{\text {loc }}$

Für Sätze mit $P P$ loc in Endstellung sieht (I) folgende Modellierung des Regelablaufs bei der Erweiterung von einfachen Sätzen durch Advb ${ }_{\text {loc }}$ vor:

- Auf der semantischen Ebene handelt es sich um ein Satzgefüge, , bestehend aus $S^{1}$ und $s^{2}$. Die Rückführung der ${ }_{\text {IOC }}$ auf $s_{\text {TS }}^{2}$ setzt dann voraus:

- Der lokalen PP wird der prädikative Ausdruck (byt' PP ${ }_{2}$ ') zugrundegelegt. Bei der Tf von $\mathrm{S}_{\text {TS }} \longrightarrow$ PP $_{\text {loc }}$ wird (byt') getilgt.

- Dieser prädikative Ausdruck (byt' PP (Oc) wird über etwas ausgesagt, und zwar - nach unserer bisherigen Analyse - über einen nominalen Ausdruck, der dem $\mathrm{N}^{1}$ von $\mathrm{S}^{1}$ referenzidentisch ist (s. jedoch Präzisierung u. S. $96 \mathrm{ff}$ ).

Bei der $\mathrm{Tf}_{2} \mathrm{CS}_{\mathrm{TS}} \longrightarrow \mathrm{PP}_{\text {loc }}$ ) wird auch dieses nominale Glied von $s^{2}$ TS getilgt.

- Model $\frac{1}{1}$ I vernaçhlässigt die Frage nach dem Satzkonnektor

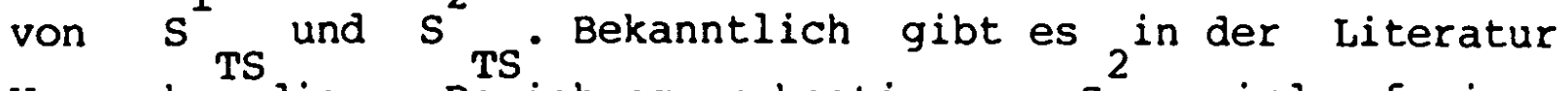
Versuche, diese Beziehung $z u$ bestimmen : $\mathrm{s}^{2}$ TS wird auf einen Nebensatz zurückgeführt, der in temporaler Relation zu S ${ }_{9}$ TS steht. 
Im folgenden wird Modell (I) mit den darin enthaltenen Analyseschritten an weiteren Beispielen mit komplexeren Satzstrukturen überprüft.

(4) Pionery videli pervogo sekretarja na stroitel'noj plošcadke.

(23) Inostrannye architektory stroili cerkov' Vasilija Blažennogo v.Moskve.

(24) Vor slyకal Šagi v komnate.

(25) Pamjatniki napominajut o partizanach $v$ selach $i$ lesach Belorussii.

(26) Knjaz' proizvel perevorot $v$ Mordasove.

Die verbalen Prädikate dieser Sätze haben Objektergänzungen, die Sätze haben die Struktur:

$\mathrm{s}_{\mathrm{OF}} \longrightarrow \mathrm{NP}^{\mathrm{I}} \mathrm{VP}\left\{\begin{array}{c}\mathrm{NP}^{2} \\ \mathrm{PP}\end{array}\right\} \quad\left(\mathrm{PP}_{10 \mathrm{O}}\right)^{\prime}$

Die Anwendung von Modell (I) ist möglich; man vgl.:

(27) Pionery videli pervogo sekretarja. Pionery byli na stroitel'noj plostadke.

(28) Inostrannye architektory stroili cerkov' Vasilija Blažennogo. Inostrannye architektory byli $v$ Moskve.

(29) Vor slysal Sagi. Vor byl $v$ komnate.

(30) Pamjatniki napominali o partizanach. Pamjatniki byli $v$ selach i lesach Belorussii.

(31) Knjaz' proizvel perevorot. Knjaz' byl $v$ Mordasove.

Die damit erhaltene explizite Bedeutung entspricht jedoch dem intuitiven Verständnis der Sätze (4), (23)-(26) nicht zur Genüge. "Auf den ersten Blick" scheinen sie vielmehr zu bedeuten: 
(32) Pionery videli pervogo sekretarja. Pervyj sekretar' by 1 na stroitel'noj ploščadke.

(33) Inostrannye architektory stroili cerkov' Vasilija Blažennogo. Cerkov' Vasilija Blažennogo (byla) v Moskve.

(34) Vor slyకal కagi. Sagi byli v komnate.

(35) Pamjatniki napominali o partizanach. Partizany byli $\mathrm{v}$ selach i lesach Belorussii.

(36) Knjaz' proizvel perevorot. Perevorot byl $\mathrm{v}$ Mordasove. Bei dieser Lesart entsprechen (4), (23)-(26) einem Modell mit folgenden Ersetzungsregeln (II):
$\mathrm{S}_{\mathrm{TS}}^{1} \longrightarrow \mathrm{NP}^{1}$
$V P\left\{\begin{array}{l}N P^{2} \\ P P\end{array}\right\}$
$\mathrm{S}_{\mathrm{TS}}^{2} \longrightarrow \mathrm{NP}_{\mathrm{n}}^{2 / \mathrm{S}^{1}}$
byt' PP Ioc

Danach wird der prädikative Ausdruck (byt' PP ${ }_{1}$ ) über das objekt von $s_{\text {TS }}$ ausgesagt. Dieses ist subjekt in dem zugrundegelegten Satz und wird bei der Tf von $\mathrm{s}^{2}$ TS $\Longrightarrow$ PP loc getilgt. Eine Folge der Tilgung ist die zu beachtende Mehrdeutigkeit des Oberflächensatzes. Die Ausführungen oben S. 63 sind dahingehend zu präzisieren, daß PP auch über andere satzglieder - als über das subjekt von ${ }_{S}^{\operatorname{loc}}$ - eine Aussage machen kann.

Die aufgezeigte Möglichkeit verschiedener Lesarten von Sätzen der Struktur wie in $(4),(23)-(26)$ ist aus der Literatur bekannt und mit verschiedenen Begriffen bezeichnet worden. So spricht man von "systembedingter Ellipse", "Kondensation" u.ä. In dem folgenden erweiterten Modell der Ersetzungsregeln (III) soll dieser strukturellen Homonymie Rechnung getragen werden: 
$s^{1} \longrightarrow N P_{\left\{\begin{array}{l}n \\ \theta\end{array}\right\}}^{1}$

$s^{2} \longrightarrow\left\{\begin{array}{c}\mathrm{NP}_{\mathrm{n}}^{1} \\ \mathrm{NP}_{\mathrm{n}}^{2}\end{array}\right\}^{\mathrm{s}^{1}}$
$V P\left\{\begin{array}{l}\mathrm{NP}^{2} \\ \mathrm{PP}\end{array}\right\}$

byt' PP IOC

Diese Mehrdeutigkeit in Sätzen mit Advb ist vorrangig durch den Typ der Prädikation, die durch (byt' PP, ) vollzogen wird, bedingt. Mit diesem Erklärungszusammenhang werden hier für die Beschreibung sprachlicher Strukturen Analysevorschläge übernommen, die in prädikatenlogischen Untersuchungen erarbeitet worden sind (Bartsch, 1972, 126). Danach gilt, daß die PP ${ }_{l}$ prinzipiell über jedes Satzglied von $S_{\text {TS }}$ ausgesagt werden kann. Ihre Anwendung ist auf die kategoriell unterschiedlichen Bereiche Dinge, Zustände und Ereignisse möglich. Bei der sprachlichen Realisierung im Russischen ist $z \mathrm{u}$ beobachten, daß die Anzahl der Lesarten von Sätzen mit PP eine variable ist, die von der stellung der $P P$ im Satz und von der Anzahl der übrigen Satzglieder loc im satz und abhängt. Endstellung der PP ${ }^{\prime}$ ' verbunden mit merkmalloser Wortfolge der übrigen Satzglieder, bedeutet in der Regel maximale Mehrdeutigkeit der PP im Satz. Dieser Umstand muß vorranging im Zusammenhang mit der Gliederung der Aussage im Russischen gesehen werden. Derzufolge ist im expressiv neutralen Satz das letzte Glied Rhema der Aussage. Unsere Analyse basiert auf der Rückführung der PP auf den prädikativen Ausdruck (byt' PP ), d.h. die fakultativen Lokaladverbiale werden als ${ }^{l 0 c}$ Prädikation verstanden. ${ }^{15}$ Im Oberflächensatz kommt bei Stellung der PP loc im Bereich des Rhema ihre tiefenstrukturelle Funktion als weitere Prädikation neben der des finiten Verbs am deutlichsten zum Ausdruck, im Unterschied zu Oberflächensätzen mit Anfangs- 
stellung der $\mathrm{PP}_{\text {loc }}$ oder Stellung $\mathrm{z} \cdot \mathrm{B}$. zwischen $\mathrm{NP}^{\mathrm{l}}$ und VP (s.u. S. 106 ff u. S. $117 \mathrm{ff}$ ).

Die Analyse der folgenden Beispiele soll diese Darlegungen verdeutlichen:

Wir nehmen zunächst den Gedanken wieder auf, daß durch die lokale Prädikation eine Aussage über kategoriell verschiedene Bereiche gemacht werden kann. In den bisher analysierten Beispielen sind es Individuenvariablen wie Personen, Dinge, denen lokale Prädikate zugesprochen werden. Für

(8) Ne konCaetsja trudovoj den' na prostorach strany.

entspricht die bisherige Anwendung des Prädikations-Tests (siehe Modell III) zur Veranschaulichung der expliziten Bedeutung kaum dem intuitiven Verständnis des Satzes:

(37) Ne konCaetsja trudovoj den'. Trudovoj den' na prostorach strany.

Das ist u.E. nicht vorrangig durch wortsemantische Faktoren bedingt - auch über Abstrakta werden lokale Prädikationen ausgesagt (s.u. Beisp. (70) ff) - sondern bei (8) handelt es sich um ein Beispiel, in dem ein "Ereignis" durch die PP näher bestimmt wird. Oberflächenstrukturell ${ }^{16}$ könnte der satz die Form haben:

(38) Net konca trudovomu dnju na prostorach strany.

d.h., daß PP über das nominalisierte Prädikat von (8) ausgesagt wird. Als Formulierung des Prädikations-Tests für die Prädikation von lokalen Adverbialen über "Ereignisse" wähle ich in Anlehnung an Bartsch ${ }^{17}$ (1972, 124):

(39) Ne koncaetsja trudovoj den'. Eto imeet mesto na prostorach strany. 
Weitere Beispiele dazu sind:

(40) Mozet projavljat'sja kakaja-to tam strast' na fone istoriðeskich sveršenij.

(41) Možet projavljat'sja kakaja-to tam strast'. Ėto imeet mesto na fone istoriðeskich sverకenij.

(42) Gost' kupil knigu v nałem kioske.

(43) Gost' kupil knigu. Eto imelo mesto $v$ nasem kioske.

(44) V. I. Dal' izučal byt, nravy 19 obycai ... dialekty russkogo naroda $\mathrm{v}$ poezdkach.

(45) V. I. Dal' izuxal byt, nravy ... russkogo naroda. Ėto imelo mesto $v$ poezdkach.

(46) Ja Zital doklad v Russkom Botaniとeskom obšestve.

(47) Ja ¿ital doklad. Éto imelo mesto v Russkom Botaniそeskom obšcestve.

(48) L. I. Brežnev podtverdil èti principy KPSS na Berlinskoj konferencii.

(49) L. I. Brežnev podtverdil èti principy KPSS. Ėto imelo mesto na Berlinskoj konferencii.

Untersuchungen der Sätze (8) und (40)-(49), die als Ergebnis die Prädikation des Lokaladverbials über das finite Verb brachten, weisen auch auf oben bereits angesprochene $\mathrm{zu}-$ sammenhänge zwischen synonymen Satzstrukturen hin. Entsprechend (38) läßt sich zu (40), (42), (44), (46), (48) bilden:

(50) Projavlenie strasti imeet mesto na fone istoričeskich sverక̌enij.

(51) Pokupka knig imeet mesto $v$ nał̌m kioske.

(52) IzuXenie byta ... russkogo naroda imeet mesto $v$ poezdkach.

(53) Ctenie doklada imeet mesto $v$ Russkom Botaniðeskom obstestve.

(54) Podtverždenie principov KPSSS L. I. Brežnevym imeet mesto na Berlinskoj konferencii. 
Die tiefenstrukturelle Modellierung erhält mit dieser Oberflächenstruktur eine empirische Bestätigung. So gesehen - bei der wahl der syntaktisch "einfacheren" Struktur mit finitem verb und PP - lassen sich (50)-(54) als Transformation von (8), (40)-(49) beschreiben ${ }^{21}$; dabei sind die wesentlichen transformationellen Beziehungen:

- fin. $\mathrm{Vb} \rightleftharpoons \mathrm{NP}$
- Advb ${ }_{\text {loc }} \Longrightarrow$ imet' mesto Advb $_{\text {loc }}$

Die grammatischen Beziehungen zwischen (8), (40)-(49) und (50)-(54) lassen sich schematisch folgendermaßen darstellen: $\left.\mathrm{NP}_{\text {nom }}^{1} \mathrm{VP}\left(\mathrm{NP}_{\text {akk }}^{2}\right){ }_{\text {loc }}^{\mathrm{PP}} \Longrightarrow \mathrm{NP}_{\text {nom }}^{\mathrm{VP}}\left(\mathrm{NP}^{2} \text { gen }\right)_{\text {instr }}\right)^{1}$

Gost kupil knigu $v$ kioske $\Longrightarrow$ Pokupka knigi gostem imela mesto $\mathrm{v}$ kioske.

In unserem Modell einer Rückführung der Lokaladverbiale auf Sätze muß der Prädikation über "Dinge" einerseits und über Ereignisse andererseits Rechnung getragen und die Ersetzungsregeln müssen entsprechend erweitert werden (IV):

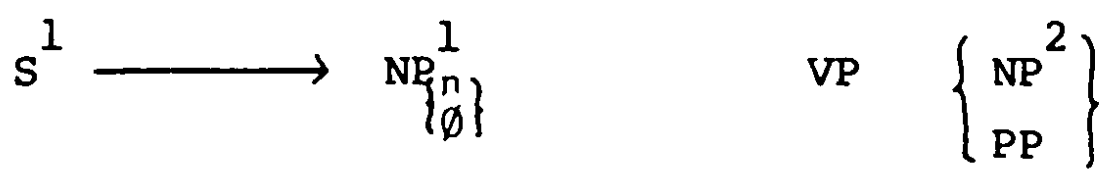

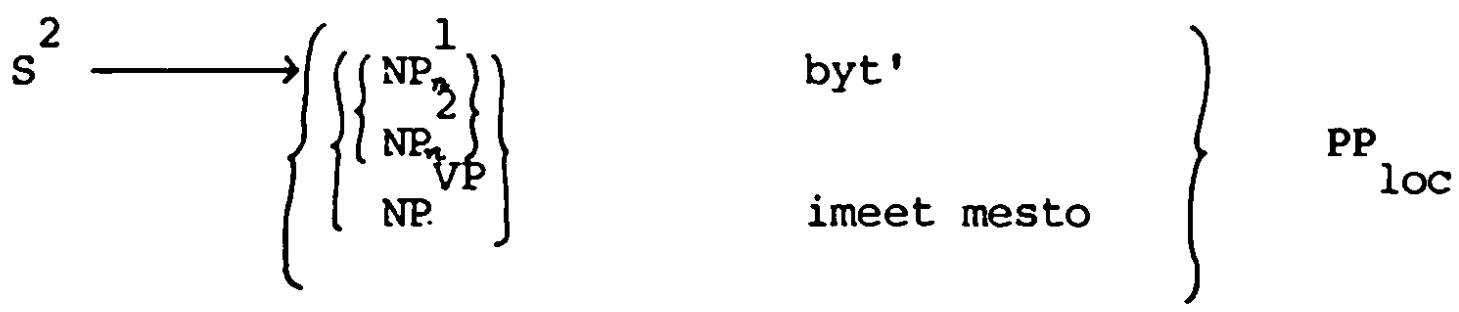

In Form eines Phrasenstrukturbaums erhalten Sätze mit fakultativen Lokaladverbialen nach dieser Analyse folgende Ableitung: 


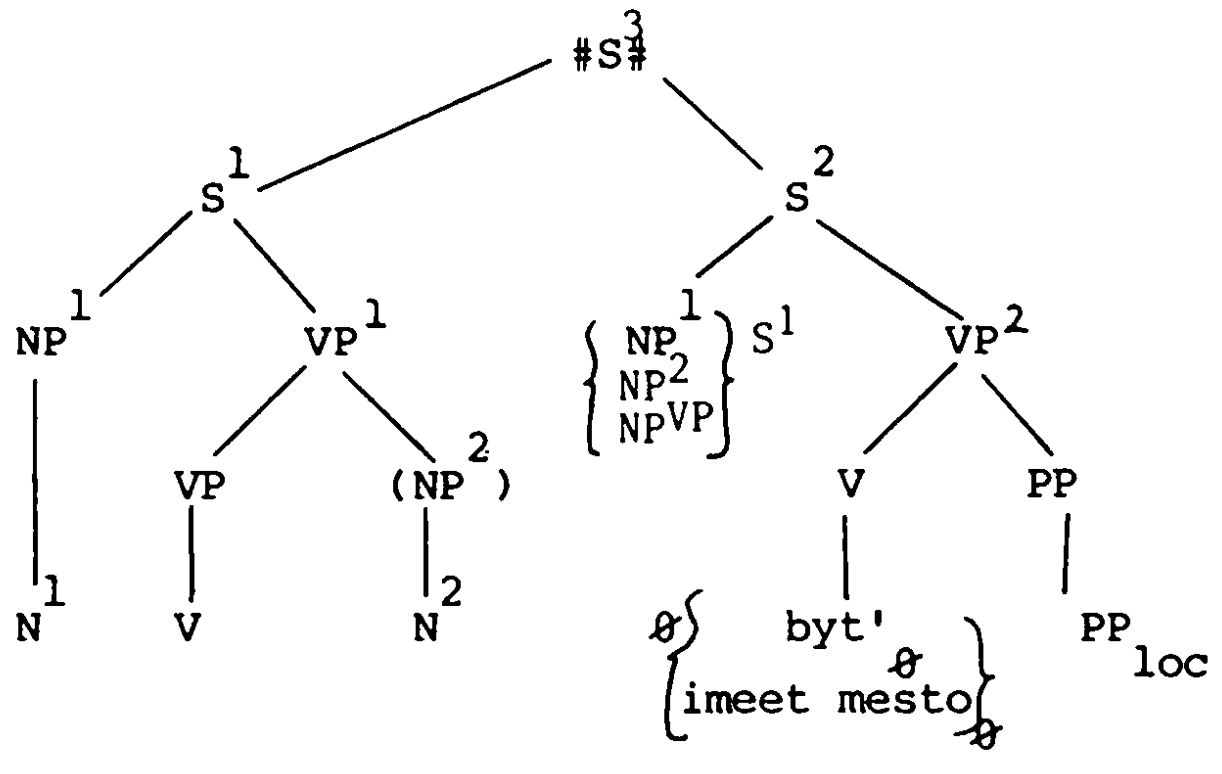

$S_{\text {TS }}$ zu:

(I) Vor isčez $v$ tolpe ljudej.

(4) Pionery videli pervogo sekretarja na stroitel'noj plošcadke.

(8) Ne kon Kaetsja trudovoj den' na prostorach strany.

Nach diesem Modell sind $\mathrm{S}^{1}$ und $\mathrm{S}^{2}$ TS Schwesterknoten, womit schematisch veranschaulicht ${ }^{\text {TS }}$ werden Soll, daß sie für die Konstitution der Satzbedeutung als nebengeordnete Elemente verstanden werden $\bar{l}$ s. dazu das folgende Kapitel über den Satzkonnektor von $\mathrm{s}^{1}$ TS ${ }^{\text {und } \mathrm{s}^{2}}$.

Mit dieser Annahme der Gleichrangigkeit von $\mathrm{s}^{1}$ und $\mathrm{s}^{2}$ TS wird in der Hierarchie der Ableitungsgeschichte ${ }^{\text {TS }}$ einerseits $S_{\text {OF }}$ mit $A d v b$ Ioc von andererseits $S_{\text {OF. }}$ mit Advb ${ }_{\text {temp }}$ und $A d v b$ mod unterschieden (s. dazu die Vorschläge Kap. temp.1.1.141 und Kap. 5.2, 202). 
3.1.2 DER SATZKONNEKTOR VON $s^{1}$ TS UND $s^{2}$ TS IM ZUSAMMENHANG MIT DER TF VON S ${ }_{\text {TS }}^{2} \Longrightarrow{ }_{\text {PP }}{ }_{\text {OOC }}$

Die Untersuchungen in Kap. 3.1.1, nämlich zu der Frage, worüber die PP ${ }_{\text {loc }}$ ausgesagt wird, führen $z u$ einer weiteren Frage: Von welcher Art ist die Konjunktion zwischen $S^{1}$ und $\mathrm{S}^{2} \mathrm{TS}^{?}$

Nach Bartsch ( $124 \mathrm{ff}$ ) ist der Satzkonnektor bei fakultativen lokalen PP temporal, den sie generell mit "gleichzeitig" bezeichnet, und zwar ohne Unterschied, ob die PP über NP in der Funktion des Agens, über $\mathrm{NP}^{2}$ in der Funktion des Patiens oder über das Prädikat von $S^{1}$ pr prädiziert wird. Steinitz sieht die Frage des Satzkonnektors als nicht so eindeutig lösbar an (op. cit. $30 \mathrm{ff}$ ). Sie hält ihre Ergebnisse zu diesem Problem für vorläufig und noch im Stadium von "Meditationen über ein Thema" (31). Ihre Analyse des Beispielsatzes

"(53) Er fand die Mark in der Hosentasche." (S. 33)

führt zu folgenden Uberlegungen:

1. Das Adverbial ist sinnvoll nur auf das Objekt beziehbar. 2. "(55) Er fand die Mark, als sie in der Hosentasche war." ist keine adäquate Paraphrase von (53).

3. Eine solche ist vielmehr:

"(56) Er fand die Mark. Sie war in der Hosentasche."

Die Uberlegungen führen zu dem "Resultat":

"Die fakultativen lokalen Adverbiale erweisen sich als 'Quasi-Lokale', die sich auf zwei verschiedene Sätze zurückführen lassen. Die subjektbezogene Adverbiale sind reduzierte Temporalsätze, die - wie wir später begründen werden - eine mögliche Expansion von $\mathrm{Adv}_{\text {Temp }}$ sind; ${ }^{\mathrm{Adv}}$ Temp 
ist Subkomponente das Matrixsatzes. Die objektbezogenen Adverbiale werden dagegen nach unserer provisorischen Analyse als Reduktion eines Satzes aufgefaßt, der dem Matrixsatz nebengeordnet ist" 10 ( $34 \mathrm{f})$.

Wie unbefriedigend die von Steinitz vorgeschlagene Lösung ist, fakultativen lokalen Adverbialen zwei verschiedene Ableitungsstrukturen zuzuschreiben, führt die verf. in der Anmerkung 10 aus. Sie gibt selbstkritisch $z u$, daß die von ihr vorgeschlagene Lösung ìu dem "unplausiblen Resultat" führt, "daß das aus dem reduzierten Satz stamende Lokaladverbial" bei Bezug auf das Objekt - "den gesamten Hauptsatz modifiziert, also als Satzadverbial fungiert. Sein Bezug auf das objekt des ersten Satzes geht dabei verloren" (187). Dieser Unstimmigkeit entgeht man, so meine ich hier noch einmal bestätigt $z u$ finden, wenn man für die sprachliche Analyse das aus der Prädikatenlogik bekannte Verfahren zur Bestimung der zugrundezulegenden Prädikation(en) heranzieht. Aufgrund der Untersuchungen $z u$ russischen fakultativen PP sind z.T. im Unterschied und in Ergänzung $z u$ Steinitz und Bartsch folgende Beobachtungen $z u$ berücksichtigen:

Wie in den bisherigen Beispielsätzen gezeigt werden konnte, kann die fakultative PP ${ }_{\text {loc }}$ prinzipiell jedes Satzglied des Oberflächensatzes, das seinerseits ein eingebetteter satz sein kann (s. dazu folgende Beispiele), näher bestimmen. Dazu gehört auch der Fall, daß die PP über "ein Ereignis", d.h. eine nominalisierte VP, präzidiert wird - dieser Fall ist m.E. bei Steinitz nicht berücksichtigt. Nach unseren bisherigen Analysen der den Sätzen mit freiem Lokaladverbial zugrundeliegenden Strukturen scheint im Fall von $(8,11,18$, $22-25,39)$ für keine der tiefenstrukturell möglichen Prädikationen die Rückführung der PP auf einen temporalen Nebensatz als explizite Bedeutung plausibel zu sein ${ }^{24}$ - man vgl.: Es sind nicht adäquat: 
zu (8): (55) *Ne kon'aetsja trudovoj den', kogda on na prostorach strany.

zu (11): (56) *Predstavitel'naja vstreła pisatelej Azii i Afriki otkryvaetsja, kogda ona $v$ Taskente.

zu (25): (57) *Pamjatniki naponimajut o partizanach, kogda pamjatniki $v$ selach $i$ lesach Belorussii.

(58) *Pamjatniki napominajut o partizanach, kogda partizany $v$ selach $i$ lesach Belorussii.

Es drängt sich die Frage auf, ob nicht andere Verhältnisse zugrundeliegen, die es erlauben, eine mehr generalisierende Regel abzuleiten, innerhalb derer unter bestimmten Bedingungen, die noch $2 \mathrm{u}$ benennen wären, $\mathrm{PP}$ loc und temporale Nebensätze als grammatische Synonyme zulässig sind. Damit würden die beiden syntaktischen Formen - PP loc und $S_{\text {temp }}$ als Strukturen verstanden, die in einem Satzableitungsschema als mögliche synonyme oberflächenrealisierungen der in Schema 1 entwickelten Tiefenstruktur einzuordnen wären. Eine Rückführung - nach Steinitz - der subjektbezogenen fakultativen $\mathrm{PP}$ loc auf temporale Adverbien wäre in diesem Erklärungszusammenhang allerdings nicht mehr schlüssig.

Bartsch (1972) bestimmt bei der Erörterung der Sätze vom Typ "Das Treffen zwischen Brandt und Stoph findet in Erfurt statt."(124) vorsichtiger als Steinitz: "Sätze dieser Art können jedoch meistens als Bildungen mit temporalem Satzkonnektor ... erklärt werden" (ibid.). Was hier "meistens" bedeutet, welches die Regeln für die Fälle sind, auf die die Bildung mit $\phi c=$ "gleichzeitig" zutrifft, und welches die Zusammenhänge sind, aufgrund derer diese Regeln nicht zutreffen, wird nicht erörtert.

Stellt man die Frage, was den hier bisher analysierten Beispielen - s. Schema 1 - gemeinsam ist, so ist die bereits erwähnte Beobachtung anzuführen, daß über ein Satzglied - bei 
mehreren NP auch über mehrere Satzglieder - neben der Aussage durch das finite Prädikat eine weitere Aussage gemacht wird, u.z. eine Aussage von der Struktur:

" $\left\{\begin{array}{l}\text { ist } \\ \text { findet statt }\end{array}\right\} \quad$ Advb ${ }^{\text {loc }}$ "

Die Beantwortung von $S_{\text {TS }}$ und $S_{\text {TS }}$ - wobei $S^{2}$ im Oberflächensatz ein freies Adverbial ist - Tä̉st unter dem Gesichtspunkt der Untersuchung sprachlicher Formen möglicherweise mehrere Antworten zu. Bei Lokaladverbialen scheint es sich um die Relation, ausgedrückt durch die Konjunktion "und" zu handeln, die die Aussagen zueinander nicht spezifiziert. Die folgenden Ausführungen sollen das illustrieren. Ich verbinde sie mit der Diskussion über die Frage, welche Faktoren für Ableitung oder Blockierung der Oberflächenstruktur PP ${ }_{\text {loc }}$ und $s_{\text {temp }}$ als grammatische Synonyme relevant sind.

1. Es können unschwer wohlgeformte Sätze, die sich nur hinsichtlich PP ${ }_{\text {loc }}$ und $S_{\text {temp }}$ unterscheiden, sonst aber lexikalisch identisch sind, temp angeführt werden, die aber Verschiedenes bedeuten. Man vgl.:

(17) Mal'と̌ik spal $\mathrm{v}$ dome storoža vodokałki.

(59) Mal'čik spal, kogda on byl $\mathrm{v}$ dome storoža vodokałki.

(21) Liza postriglas' $v$ B... om monastyre.

(60) Liza postriglas', kogda ona byla v B... om monastyre. Dagegen verstehen wir im Unterschied $z$ diesen Beispielen die folgenden Satzpaare als grammatische Synonyme; man vgl.:

( 4) My videli pervogo sekretarja obkoma na stroitel'noj plostadke.

(61) My videli pervogo sekretarja obkoma, kogda

my

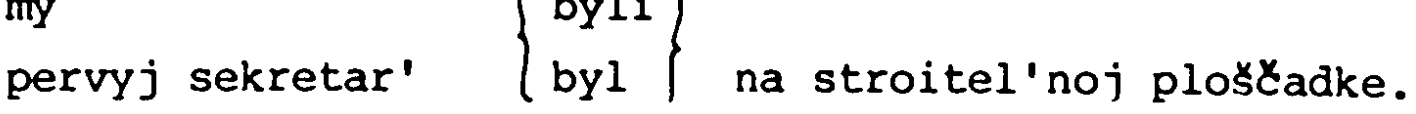


(44) V. I. Dal' izučal byt, nravy, obyčai i dialekty russkogo naroda, $v$ poezdkach.

(62) V. I. Dal' izuð̌al byt, nravy, obycai i dialekty russkogo naroda, kogda on byl $\mathrm{v}$ poezdkach.

(48) L. I. Brežnev podtverdil éti principy KPSS na Berlinskoj konferencii.

(63) L. I. Brežnev podtverdil éti principy KPSS, kogda on byl na Berlinskoj konferencii.

Wodurch ist der Unterschied bedingt? M.E. ist eine relevante Bedingung in dem Faktor "Zeit" (nicht Tempus), soweit er in Aussagen - oft implizit - bezeichnet wird, zu sehen. Die beiden Prädikate in den tiefenstrukturell angenommenen Sätzen $\mathrm{S}_{\mathrm{TS}}$ und $\mathrm{S}^{2}$ implizieren durch ihre lexikalische Bedeutung auch den Begriff "Zeit", z.B. als "Zeitraum", "Zeitpunkt" etc. Oberflächensätze mit PP bedeuten, daß das Verhältnis von $\mathrm{Vb}^{1}$ und $\mathrm{vb}^{2}$ der TS nicht näher bestimmt wird, entsprechend wird hier tiefenstrukturell die Konjunktion "und" angenommen. Dagegen wird in Sätzen mit $s_{\text {temp }}$ offensichtlich in unterschiedlicher weise ${ }^{26}$ - das zeitliche verhältnis von $\mathrm{Vb}^{1}$ und $\mathrm{Vb}^{2}$ fixiert, ausgedrückt durch die Konjunktion "kogda". In den Fällen nun, in denen "Zeit", "zeitliche Grenze" etc. von $\mathrm{Vb}$ und $\mathrm{Vb}^{2}$ aufgrund ihrer lexikalischen Bedeutung als einander entsprechend verstanden werden, spezifiziert die Konjunktion "kogda" nicht mehr, als was auch mit der Konjunktion "und" mitverstanden wird, nämlich die präsupponierte Linearität aller sprachlichen Außerungen. Das ist in den zitierten Beispielen der Fall bei $(4) /(61)$, $(44) /(62)$ und $(48) /(63)$. Diese Bedingung ist nicht erfüllt 2.B. in $(17) /(59)$ und $(21) /(60)$. Den Unterschied kann man umschreiben mit: während (17) bedeutet: der Junge übernachtet in dem Haus ..., bedeutet (59), daß der Junge schlafend in dem Haus ... war. (21) bedeutet, daß es das Kloster B... war, in dem Liza zur Nonne geschoren wurde; dagegen bedeutet (60), daß Liza in (verschiedenen) Klöstern war und daß sie in dem B... Kloster zur Nonne geschoren wurde. 
2. Diese Versuche, den Unterschied z.B. von (17) $\mathrm{zu}$ (59) und (21) $\mathrm{zu}$ (60) $\mathrm{zu}$ verdeutlichen, weisen auf einen weiteren Faktor hin, nämlich die Thema-Rhema-Gliederung, die als regelbestimmend für die verwendung von $P P_{\text {loc }}$ und $s_{\text {temp }}$ als grammatische Synonyme gesehen werden muß. Auch hier mus ich mich auf vorläufige Beobachtungen beschränken, da Untersuchungen zu Temporalsätzen unter diesem Gesichtspunkt m.W. nicht vorliegen. Es wird unten Kap. 3.2.1 ( $89 \mathrm{ff})$ ausgeführt werden, daß PP, in Finalstellung für die Aussage in der Regel funktionale Mehrdeutigkeit bedeutet - soweit nicht andere Faktoren disambiguierend wirken - d.h. daB PP zum Bereich des Rhema gehört oder Rhema proprium ist. Damit ${ }^{10 C}$ wird die Frage nach der "Grenze" zwischen Thema und Rhema für die Gliederung der Aussage relevant, die u. (S. $87 \mathrm{ff}$ ) behandelt wird. Diese Mehrfunktionalität scheint auf $S_{\text {temp nicht }}$ übertragbar zu sein, woraus folgt, daß die Thema-RhemaGliederung in den hier verglichenen Satzpaaren verschieden sein kann. Das ist der Fall bei (17) und (59): während in (17) die VP mit PP loc oder nur PP loc Rhema sein können, gilt dies nicht für (59): Rhema ist hier nur "byl $v$ dome storoza vodokacki."

Eine weitere Bestätigung für die wichtigkeit der Thema-RhemaGliederung im Hinblick auf die hier diskutierte Frage gibt die Bedeutung von $(60)$ : denn $(60)$ in der Bedeutung von (21) wäre eine "leere Aussage": zur Nonne geschoren werden kann man nur im Kloster. Ahnlich verhält sich:

(12) Veselye gosti Sumno razdevalis' $v$ perednej.

Dazu scheint aus vergleichbarem Grund wie bei (60) nicht akzeptabel:

(64) *Veselye gosti Sumno razdevalis', kogda oni byli $v$ perednej.

im Unterschied $z u$ : 
(65) Veselye gosti gromko razgovarivali, kogda oni byli v perednej.

Hier sind m.E. die Voraussetzungen für eine synonyme Verwendung von $S_{\text {temp }}$ und PP $_{\text {loc }}$ gegeben; man vgl.:

(66) Veselye gosti gromko razgovarivali $v$ perednej.

3. Gegenüber den in 1. und 2. genannten Faktoren ist die lexikalische Besetzung der NP von $S{ }_{T S}$, die bisher die Aufmerksamkeit der Forschung auf sich gezogen hat, von untergeordneter Bedeutung. Darüberhinaus ist es nicht der Faktor "subjektbezogenes Adverbial" 28 , der hier eine Rolle spielt, sondern wohl das semantische Merkmal "+ belebt" der NP, über die die Aussage "NP ist Advb loc" gemacht wird; man vgl. dazu oben S. 74 das Satzpaar $(4) /(61)$.

Die kritische Auseinandersetzung mit der These, daß PP tiefenstrukturell auf temporale sätze zurückzuführen seien, führten 1. zu dem Ergebnis, daß nur unter bestimmten Bedingungen, die unter 1 - 3 grob erarbeitet wurden, PP und $s_{\text {temp }}$ als grammatisch synonyme oberflächenstrukturen verwendet werden können. Denn während in Schema $1_{2}$ für PP tiefenstrukturell die Konjunktion von $s$ und $s$ "und" ist, deuten die vorläufigen Untersuchungen der Gegenüberstellung von $P P$ loc und $S_{\text {temp }}$ darauf hin, das bei der Ableitung von $S_{\text {temp }}^{\text {loc }}$ in der Regel Restriktionen zu beachten sind, die die Relation von $s^{1}$ und $s^{2}$ rs zeitlich spezifizieren; 2. führen die dargestellten verhältnisse von möglichen grammatisch synonymen Oberflächenstrukturen (hier PP ${ }_{10 c}$ und $S_{\text {temp }}$ ' $z u$ der Frage, ob es noch andere grammatisch (quasi-)synonyme Oberflächenstrukturen zu Sätzen mit PP gibt, in denen die Bedingungen $1-3$ ebenfalls nicht regelmäBig gegeben sind. Das scheint der Fall zu sein; zum einen kann das der Relativsatz sein , man vgl.: 
(25) Pamjatniki napominajut o partizanach $v$ selach $i$ lesach Belorussii.

(67) Pamjatniki napominajut o partizanach, kotorye byli $v$ selach i lesach Belorussii.

(68) Deti smotreli na snega za oknami.

(69) Deti smotreli na snega, kotorye byli za oknami.

Zum anderen erhalten wir bei Prädikation über VP nach Nominalisierungs-Tf die Konstruktion:

(38) Net konca trudovomu dnju na prostorach zemli.

In Ergänzung $z u$ diesen Beobachtungen behandelt der folgende Abschnitt das Verhältnis der PP ${ }_{\text {loc }}$ zu anderen Tf des Satzes. 
3.1.3 DAS VERHÄLTNIS DER Tf $\mathrm{s}^{2}{ }_{\mathrm{Tf}} \Longrightarrow{ }_{\text {PP }}{ }_{\text {lOC }}$ ZU ANDEREN Tf DES SATZES

Bei der Untersuchung der Frage, über welche Satzglieder die fakultative PP loc prädiziert wird, stößt man auf Sätze, in denen die PP ${ }_{\text {loc }}$ entweder über andere fakultative PP oder über andere durch ${ }_{\text {Tf }}^{10 c}$ abgeleitete Satzglieder prädiziert wird. Für die Derivationsgeschichte 2 von Sätzen mit fakultativer PP bedeutet das, daß die $\mathrm{Tf}^{2}{ }_{\mathrm{Tf}} \Longrightarrow{ }_{\mathrm{PP}}{ }_{\text {loc }}$ auf einer relativ niedrigen Stufe - d.h. relativ oberflächennah - der satzableitung anzusetzen ist und sie ihrerseits andere Tf nicht blockiert. Als Beispiele dazu:

die PP ${ }_{\text {loc }}$ bezieht sich auf andere fakultative PP:

(70) Ja vspominaju ob ètom $s$ ulybkoj $v$ duse.

Die PP "s ulybkoj" ist ein Modaladverbial und ihrerseits ein Ergebnis von Transformationen. Als zugrundeliegende Satzstruktur von (70) formulieren wir vorläufig:

(71) Ja vspominaju ob ètom. Ja ulybajus'. Ėto imeet mesto $v$ duše.

Die fakultative PP "v duse" wird damit über eine VP prädiziert, die selbst "neben" einer anderen VP NP näher bestimmt. Dabei ist festzustellen, daß in dieser Funktion das Lokaladverbial vornehmlich in Endstellung steht.

Beispiele wie ( $70 \mathrm{ff})$ geben m.E. eine weitere objektsprachliche Bestätigung des entwickelten Modells, das in den PP ${ }_{\text {loc }}$ ein Mittel prozessualer Aufbauprinzipien von Sätzen sieht: es werden Aussagen, oberflächenstrukturell "verkürzt", in der kondensierten Form des Lokaladverbials aneinandergereint. 
Das Lokaladverbial bestimmt dabei oft das unmittelbar vorhergehende Satzglied, das z.B. eine PP unterschiedlicher Klassen sein kann:

(72) Varvara Pavlovna rešilas' ne davat' mne Zit' - podumal on $s$ volneniem zloby na lice.

(73) Vse èto ona delala ne speša ... s kakoju-to zabotlivost'ju na lice.

(74) ... potomu ťto lišilsja gde-to rebra, ... vo vremja odnogo svoego ljubovnogo pochoždenija, v Italii.

(75) ... utverždali, ... tto nastojašxuju nogu slomali $\frac{1}{3}$ emu, pri kakom-to drugom pochoždenii, v Pariže.

In Sätzen wie (70-73) ist abstrakte Lexik verbreitet. "Lokaladverbiale" haben hier ihre primäre Bedeutung verloren, nämlich ein konkretes "Ding" oder ein intersubjektiv wahrnehmbares "Ereignis" "an", "vor", "hinter" etc. einer bestimmten stelle zu lokalisieren. Bei der verwendung von Lokaladverbialen in figurativem sinn kann man bestimmte Beobachtungen machen, die die Lexik und Grammatik dieser Sätze betreffen. Zum einen gehören - wie gesagt - die Nomina, über die die Lokalaussage gemacht wird, zur Klasse der Abstrakta; des weiteren ist die Präposition der PP in der Regel nicht substituierbar. Damit entfällt ein formales Kriterium für 33 die Unterscheidung der verschiedenen Klassen der Adverbiale . Wortbildungsregeln ${ }^{34}$, wie Ableitung der Abstrakta, können die Lesarten der Sätze in der weise variieren, daß oberflächenstrukturell der Unterschied z.B. von attributiver und adverbialer Prädikation neutralisiert ist. Das soll Beispiel (81) demonstrieren; "pevukest'", abgeleitet von "pet'", hat keine inhärenten verbalen semantischen Merkmale mehr ${ }^{36}$ :

(81) ... voskliknul on so svojstvennoju emu poryvistoj pevucest'ju $v$ golose. 
Geht man von dieser Oberflächenform des Satzes aus, sind verschiedene Einstiege für seine Analyse denkbar: man kann mit der Analyse der 1. PP oder mit der Analyse der 2. PP beginnen. Ausschlaggebend für die Bestimmung der grammatischen Funktionen seiner Glieder ist jedoch der Umstand, da $B$ zu Ableitungen grammatischer Funktionen Wortbildungsableitungen hinzukommen - auf diesen Komplex kann ich in dieser Arbeit nur gelegentlich verweisen.

Nach Zolotova (loc. cit. 136) ist für die verwendung der von Adjektiven abgeleiteten Nomina charakteristisch, daß sie in "polyprädikativen" Satzstrukturen verbreitet sind. Dabei tritt das Nomen nicht in eine unmittelbare prädikative Beziehung zu der Bezeichung des Gegenstandes, sondern es bedarf dafür Hilfswörter. Als solche Hilfswörter werden z.B. angeführt: "prisuš, svojstven, tipiŁ̌en, charakteren". In so konstruierten Sätzen steht "das Nomen des Gegenstandes, über den prädiziert wird, im Dativ oder Genitiv mit der Präposition dlja. (Materialu prisušca, svojstvenna elastixnost', ...)" (ibid.).

Eine weitere Oberflächenstruktur dieses Satztyps ist m.E. (81) mit dem Nomen im Präpositiv.

Eine Folge der verschiedenen Typen von Ableitungen - hier Wortbildung und grammatische Funktionen - ist, daß für diese Art komplexer strukturen eine Reihe syntaktisch synonymer Oberflächensätze gebildet werden kann. Man vgl.:

(82) ... voskliknul on pevuce. (Pevuce voskliknul on.)

(83) ... voskliknul on pevučim golosom.

(84) ... voskliknul on s pevuxest'ju v golose.

(85) ... voskliknul on s poryvistoj pevukest'ju (svoego) golosa.

Oberflächenstrukturell entspricht auch (86) dem Beispiel (81), jedoch ergibt die Untersuchung auf erfolgte Tf einen anderen Regelablauf für die Ableitung dieses Satzes. 
(86) Gor'kij protiv vosproizvedenija rexi malogramotnych v literature.

"protiv vosproizvedenija" ist nach der Nominalisierungs-Tf eines Satzes abgeleitet, der als Objektsatz von einem Satz des "Meinens über etwas" abhängt:

(87) Gor'kij protiv Cego-to. Vosproizvoditsja rex' malogramotnych $v$ literature.

Die fakultative PP loc wird über die VP des Objektsatzes prädiziert.

(88) Gor'kij protiv Cego-to. Vosproizvoditsja rec' malogramotnych. Éto imeet mesto $v$ literature.

Auch Infinitivsätze können durch Lokaladverbiale erweitert werden. In (89) und (92) entsprechend ist der Infinitivsatz von einem Objektsatz abgeleitet.

(89) Sestra prosila brata ne zabyvat' ee na cužbine.

Satz ( 89 ) ist mehrdeutig. ${ }^{37}$ Er kann bedeuten:

(90) Sestra prosila brata, ttoby on ne zabyl ee, kogda on na cužbine.

und

(91) Sestra prosila brata, ttoby on ne zabyl ee, kogda ona na cužbine.

Die bisherigen Analysen wurden vorrangig mit dem ziel der Auffindung und des Nachweises der Grundfunktion lokaler Adverbiale geführt. Das empirische Material weist auf die Prädikation - eine (weitere) Prädikation(en) neben der durch das finite Verb - als Grundfunktion hin. Für die Beschreibung 
der vorliegenden sprachlichen Strukturen muß diese Grundfunktion zusammen mit bestimmten formalen Merkmalen der Sätze mit Adverbialen gesehen werden, z.B. der systembedingten Ellipse. Diese semantischen und syntaktischen Verhältnisse in Sätzen mit lokalen Adverbialen führen zu vielfacher und durch unterschiedliche Faktoren bedingter Mehrdeutigkeit. Es drängt sich die Frage auf: welche Faktoren sind es, die gewährleisten, daß die sprache - auch hier ihrer Mitteilungsfunktion - nämlich der Verständigung der Gesprächspartner zu dienen - gerecht wird? Dieser Frage ist im wesentlichen Kap. 3.2 gewidmet. Hier sollte auf grammatische Faktoren hingewiesen werden, die im Zusammenhang mit $\mathrm{Tf} z \mathrm{u}$ sehen sind.

Die Ableitung von $s^{2} \longrightarrow{ }_{\text {TS }} \Longrightarrow$ erfolgt in Sätzen mit VP nach Regeln, dìe denen in den Sätzen mit VP entsprechen. Die Anzahl der möglichen Lesarten durch aktiv Prädikation von $\mathrm{PP}$ loc über die übrigen Satzglieder ist wohl in der Regel dann verringert, wenn $\mathrm{NP}_{n}$ des zugrundegelegten aktiven Satzes durch die Passiv-Tf getilgt ist. Man vgl. oben:

(11) Predstavitel'naja vstreca pisatelej Azii i Afriki otkryvaetsja $v$ Taskente:

"v Tałkente" kann hier nähere Bestimmung zu "vstreč" und/oder "otkryvaetsja" sein. Naheliegend ist die Lesart, die nach Nominalisierungs-Tf folgender oberflächensatz erhält:

(95) Otkrytie predstavitel'noj vstrexi ... imeet mesto v Tałkente.

Eine entsprechende Beschreibung gilt für (96) und (97), ohne daß man jedoch analoge Oberflächensätze bilden kann, was wiederum auf Wortbildungsregeln zurückzuführen ist; man vgl.: 
(96) ... glavnaja pobeda nad fasizmom byla oderžana na Vostoknom fronte.

(97) Eto otlitie vidno ... v takich primerach.

Vergleichbar dieser Restriktion der Prädikationsbeziehungen von PP ${ }_{l o c}$ ist diejenige in unpersönlichen Konstruktionen, z.B. in (98) im Unterschied zu oben (4):

(98) Načal'nika politotdela 18-oj armi i možno bylo uvidet' na samych opasnych ucastkach fronta.

Es gilt jedoch folgenden Fall von Passivkonstruktion hier zu erwähnen, der in das bisher dargelegte Schema nicht paßt. Es geht um Sätze der Art wie:

(99) Na nekotorych zarubežnych firmach daže కtatnaja edinica predusmotrena - myslitel'.

Entgegen der Beschränkung dieses Kapitels auf Beispielsätze mit der PP in Endstellung halten wir es für angebracht, diesen Satz hier zu erörtern. Ein weiterer Beleg ist:

(100) ... Na "Trechgorke" vypuskajutsja uzornye tkani dlja ... sorocek.

Als Passivkonstruktion fallen diese Sätze dadurch auf, daß einerseits bei der Bildung der ihnen entsprechenden Aktivkonstruktion die ${ }_{\text {PP }}$ loc die subjektstelle besetzen kann; man vgl.:

(101) Nekotorye zarubežnye firmy predusmatrivajut daže Statnuju edinicu - myslitelja. -

Andererseits ist die übliche Passiv-Tf von (101) insoweit blockiert, als die Subjekt-NP "Nekotorye zarubeznye firmy" 
("Trechgorka") nicht als $\mathrm{NP}_{\text {instr }}$ erscheinen, sondern als PP $10 c^{\circ}$

Diese Verwendung von PP ${ }_{40}$ in Passivsätzen untersucht I. zimmermann und kommt $z u$ dem Schluß, daß man zwischen "ursprünglicher" und "abgeleiteter" Transitivität in Aktivsätzen zu unterscheiden habe. In (101) haben wir ein Beispiel für abgeleitete Transitivität: die Subjektstelle wird von einer NP eingenommen, die von einer $P P$ loc in den zugrundegelegten Strukturen, dem Passivsatz und dem Aktivsatz mit NP $\theta^{\prime}$ abgeleitet ist. 
3.2 PRÄDIKATION DURCH PP ${ }_{\text {IOC UND AKTUELLE SATZPERSPEKTIVE }}$

Für die generativ-transformationelle Analyse der Funktion von Lokaladverbialen im Satz wurden bisher Sätze mit PP $10 \mathrm{c}$ in Endstellung gewählt. Dies erschien deshalb zweckmäßig, da auch andere Untersuchungen darauf hindeuten, daß in dieser Oberflächenrealisierung des Satzes entsprechend der aktuellen Gliederung der Aussage im Russischen die Grundfunktion der Adverbiale - nämlich eine weitere Prädikation zu sein - am deutlichsten zum Ausdruck kommt (s.0. Kap. 3.1 .1 bes. S. 67 ff).

Als Argumente des zugrundegelegten lokalen Prädikats können wie gezeigt wurde - im Prinzip alle nominalen und verbalen Glieder des Satzes fungieren. Aus der Tf von $\mathrm{S}_{2} \longrightarrow$ PP folgt für den Oberflächensatz eine potentielle Mehrdeutigkeit.

Es sind verschiedene Faktoren $z u$ beobachten, die die in den Kap. 3.1.1 und 3.1.3 besprochene und an Beispielen demonstrierte strukturelle Homonymie einschränken bzw. Mehrdeutigkeiten im oberflächensatz disambiguieren. Hier ist an erster Stelle der Faktor "Vorwissen" $"$ zu nennen. In:

(102) Rebenok narisoval obez'janu v kletke.

kann "v kletke" "vernünftigerweise" nur auf "obez'jana" bezogen werden. Als weiterer Faktor muß der Kontext genannt werden, der disambiguierend wirkt, vgl. oben (92).

Restriktionen entstehen bei semantischen Merkmalentsprechungen zwischen einzelnen Satzgliedern, z.B. zwischen Verb und Präposition der PP; man vgl.:

(103) On vystupil s dokladom na veerasnem sobranii. (104) ... i vystupil on s dokladom na věerasnem sobranii. 
im Unterschied zu:

(105) Uðenye prinesli obrazcy mineralov s luny.

(106) Prinesli uXenye obrazcy mineralov s luny.

Auf grammatische Faktoren wurde bereits hingewiesen (s. oben $83 \mathrm{fl}$ ).

Ausführlicher möchte ich in dieser Arbeit die "funktionale Satzperspektive" 42 untersuchen, da sie bei der Bildung eines jeden Satzes - wie gezeigt wird - zu berücksichtigen ist, während die übrigen Faktoren von mehr oder weniger umfassender Relevanz sind.

Für die Berücksichtigung der "Topic Comment Articulation" im Hinblick auf die Prädikationen durch PP ${ }_{\text {IOC }}$ wird der bisherige generativ-transformationelle Beschreibungsansatz erweitert, indem Beschreibungsprozeduren und -ergebnisse in unsere uberlegungen einbezogen werden, die aus den Untersuchungen zur aktuellen satzperspektive hervorgehen ${ }^{4}$.

Die einleitenden Beispielsätze (1-13, S. oben S. 20 f), machten offenkundig, daß für die Ableitung eines Oberflächensatzes mit einer PP das Problem der Prädikation durch das Lokaladverbial mit der Thema-Rhema-Gliederung des Satzes verbunden ist. Es wird sich zeigen, daß dabei von nicht unerheblicher Bedeutung die Frage ist, welchem Bereich - dem des Themas, dem des Rhemas oder keinem von beiden eindeutig das Lokaladverbial zuzuordnen ist.

Mit dieser allgemeinen Problemstellung ist eine speziellere verknüpft, der ebenfalls in den $T-C-A-A n a l y s e n$ eine Reihe von Arbeiten gewidmet wurde, nämlich den Fragen:

1. Inwieweit ist die Grenze zwischen Thema und Rhema für die Bedeutung der Aussage relevant? (Sgall, 1976; Sgall, Hajičová, Benešová, 1973) 
2. Wo ist der "Schnitt" in der Gliederung der Aussage anzusetzen, und welche Kriterien gibt es für dessen Feststellung (vgl. auch Danes, 1968)?

Die folgende Analyse der lokalen Adverbiale unter dem Gesichtspunkt der Thema-Rhema-Gliederung der Aussage geht der Frage nach, ob Regelmäßigkeiten für die Auflösung der strukturellen Homonymie durch Restriktionen, bedingt durch die funktionale Satzperspektive, $z u$ beobachten sind - sie hat nicht die Absicht, einen Beitrag zu der Rolle der Adverbiale innerhalb der T-C-A überhaupt $z$ u leisten. 
3.2.1 SÄTZE MIT PP ${ }_{1 O C}$ IN ENDSTELLUNG

Wie die generativ-transformationelle Analyse der Beispiele in Kap. 3.1.1 zeigt, hängt die Anzahl der potentiellen Lesarten von Sätzen mit PP ${ }_{\text {loc }}$ von der Anzahl der übrigen Satzglieder $a b$ - je größer deren Anzahl ist, um so größer sind die Möglichkeiten der Prädikation durch PP ${ }_{\text {loc }}$ in Endstellung (vgl. Jaksche, 1978).

Andererseits haben die Untersuchungen zur $T-C-A$ ebenfalls ergeben, daß funktionale Mehrdeutigkeit von Sätzen immer dann zu beobachten ist, wenn das finite prädikat eine objektergänzung hat (Daneš, 1968; Uhlířová, 1976), dabei wird von der "Grundform" der Aussage, der "paradigmatisch starken" oder "kontextfreien" ausgegangen.

Es sei kurz die Argumentation resumiert: Auf der Suche nach überprüfbaren kriterien für die Bestimmung des Rhema erkannte man als ein wichtiges Kriterium den Frage-Test. Semantisch ist die Frage in der Weise aufgebaut, daß sie "Vorausgesetztes" nennt und - mit Hilfe von Fragepronomen und/oder Intonation und Wortstellung - nach dem fragt, was das "Vorausgesetzte" näher bestimmen soll, das seinerseits ziel der Aussage, Rhema ist. Anders gesagt, eine Aussage hat funktional die Struktur einer zweiteilung, von der der eine Teil (thematischer Teil) so formuliert wird, daß er mit dem Fragewort bzw. durch die Intonation und wortstellung nach dem fragt, worauf der zweite Teil (rhematischer Teil) eine Antwort geben soll.

In Sätzen, in denen das finite Prädikat eine objektergänzung hat, sind immer mindestens 2 Fragen möglich - im Unterschied zu Sätzen, in denen das Prädikat absolut gebraucht wird:

1. Es wird nach der verbalen Phrase im ganzen gefragt, indem man das finite prädikat durch ein verb mit einer ganz 
allgemeinen Bedeutung ersetzt: "Was tut $x$ ?"; Rhema ist dann das finite Verb einschließlich Verbergänzung; oder

2. Es wird nach der Verbergänzung gefragt: "Was - fin. Präd.

- $x^{n}$; Rhema ist hier die Verbergänzung.

Im weiteren interessiert hier die Frage: Ergeben sich regelhafte Restriktionen für die Prädikation durch PP oder Veränderungen der syntaktischen Verhältnisse in der oberflächenrealisierung aufgrund der T-C-A? Anders gefragt:

Gibt es eine Interrelation zwischen den Faktoren, die syntaktisch wirken - hier beschränkt auf die Erweiterung der

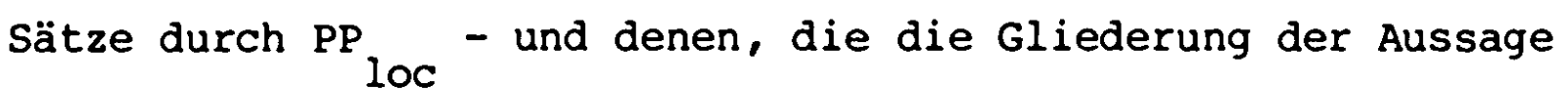
regeln? Und lassen eventuelle Ergebnisse Schlüsse zu diesem Problem auch im Hinblick auf andere Oberflächenrealisierungen wie Anfangsstellung der PP ${ }_{\text {loc }}$ oder andere Aussagestrukturen (wie "syntagmatisch stark") 100 ?

Für die folgenden Untersuchungen entwickelte ich den ThemaRhema-Gliederungs-Test, der auf dem oben erwähnten Frage-Test aufbaut. Dabei gehe ich folgendermaßen vor:

1. Ein Satz in der kontextfreien Form der Aussage ( ${ }_{1 \mathrm{KF}}$ ) wird mit Hilfe von Fragen $\left(Q_{n-1}\right)$ auf seine möglichen funktionalen Gliederungen untersucht. Ich gehe von sätzen der struktur $\mathrm{S} \longrightarrow \mathrm{NP}{ }^{1}$ VP NP ${ }_{45}^{2}$ aus, diesè werden durch PP ${ }_{\text {loc }}$ erweitert $^{44}$. 2. Es wird eine ${ }^{45}$ kontextabhängige Aussageform von $S_{1}$ ( $S_{1 K S}$ ) gebildet, diese wird ebenfalls durch PP erweitert und entsprechend analysiert. Danach werden die funktionalen Gliederungen der erweiterten $S_{\text {IKF }}$ und $S_{\text {IKS }}$ verglichen.

Der Test kann überprüfen, ob die grammatischen Beziehungen der Satzglieder innerhalb des thematischen und rhematischen Teils bei der kontextfreien und kontextabhängigen Form der Aussage identisch bleiben, da bekanntlich (Kovtunova, 1976, im besonderen Kap. II) grammatische (und wortsemantische) Invarianz eine Voraussetzung fUr das funktionale Paradigma 
von Aussagen sind. Von dieser Uberprüfung können Kriterien für die Beantwortung der gestellten Frage erwartet werden: Spielt der funktionale Faktor bei der Disambiguierung von Sätzen mit PP ${ }_{\text {loc }}$ eine relevante Rolle und wenn ja, wie ist er zu bestimen?

Für die Untersuchung von PP ${ }_{l o c}$ unter funktionalem Gesichtspunkt wurde die Gegenüberstellung von kontextfreien und kontextabhängigen Aussagen deshalb gewählt, weil, wie aus vorliegenden Arbeiten hervorgeht (Jaksche, 1978), die funktionalen Gliederungsmöglichkeiten in kontextabhängigen Aussagen in der Regel eingeschränkter sind als in kontextfreien, d.h., solange es sich um Aussageformen eines funktionalen paradigmas handelt, kann $s_{\text {lKs }}$ nicht mehr "Lesarten" enthalten als $S_{1 K F}$. Daraus folgt - anders gesagt daß, wenn wir mit dem Test Thema-Rhema-Gliederungen erhalten, innerhalb derer sich die grammatischen Beziehungen von $\mathrm{S}_{1 \mathrm{KF}}$ und $S_{1 K S}$ nicht entsprechen, es sich um sätze handelt, die nicht zu demselben funktionalen Paradigma gehören; diese Sätze bedürfen einer weiteren Untersuchung. Vorwegnehmend sei hier auf $\mathrm{Th}_{6} / \mathrm{Rh}$ und $\mathrm{Th}_{7} / \mathrm{Rh}_{7}$ der kontextabhängigen Form verwiesen ( $s^{6}$. folgende Seiten, z.B. die Ergebnisse des Tests zu (25), (44) etc.). Wie o. bereits erwähnt, beschränkt sich diese Arbeit auf die Untersuchung expressiv neutraler Aussagen. Damit handelt es sich um Sätze mit "normaler" (neutraler) Intonation, die durch $e$ i $n$ Intonationszentrum gekennzeichnet sind. Die Grenze von Thema und Rhema, dargestellt durch "/", markiert die Intonationspause.

Zur Demonstration des Tests mögen folgende Beispiele dienen: 


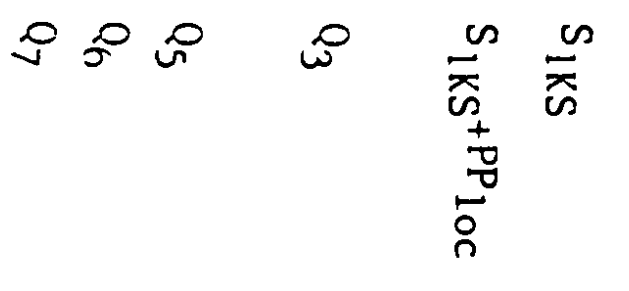

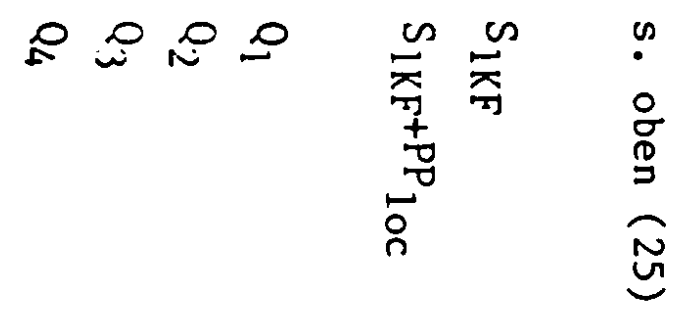
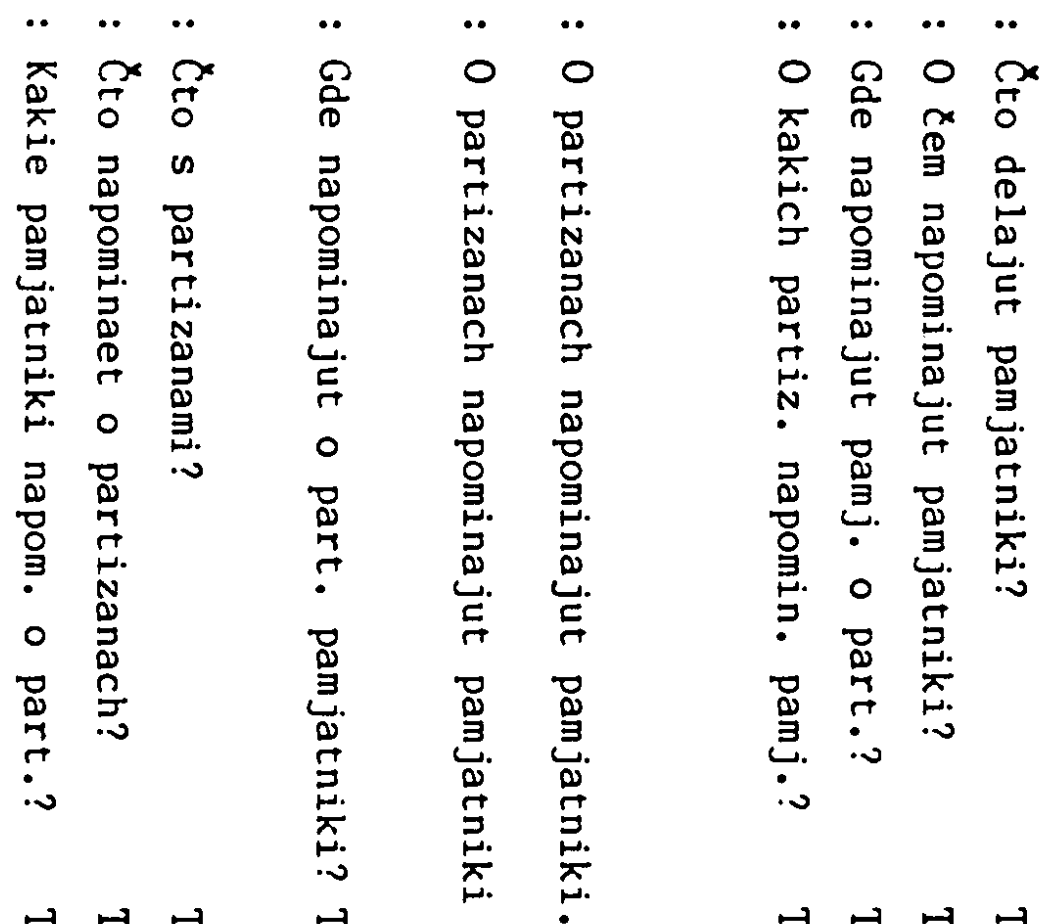

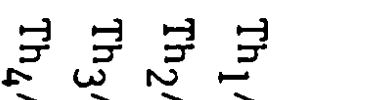

兽. 惫

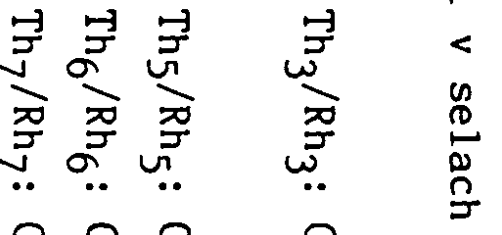

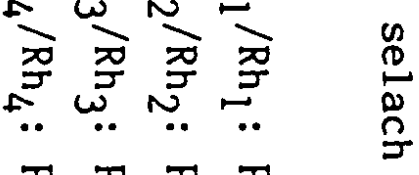

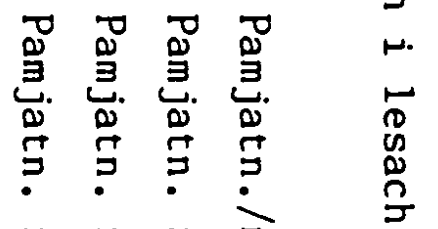

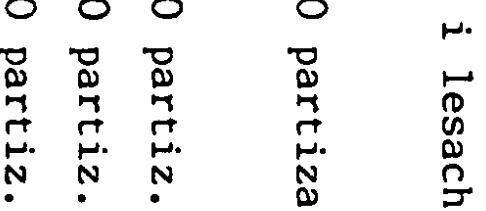

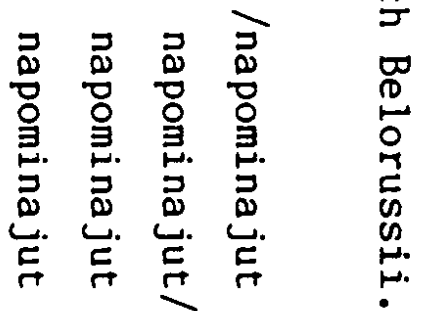

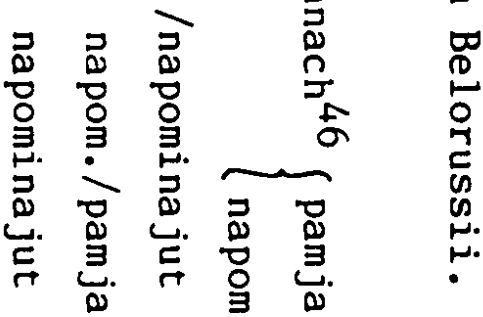

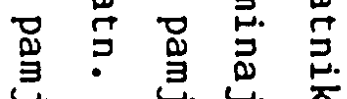

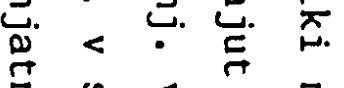

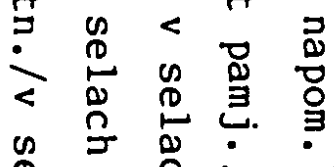

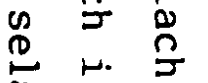

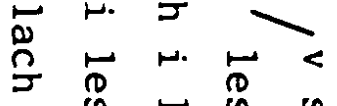

‥

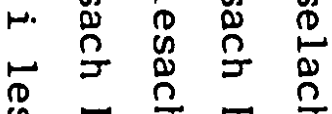

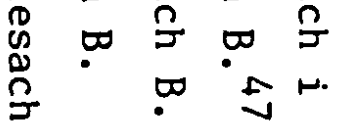

- 0 응

品 品

? ? ? ? ? ? ?

$<<<$

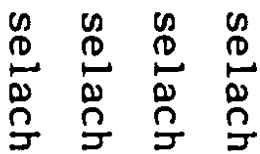

‥ ‥ ‥

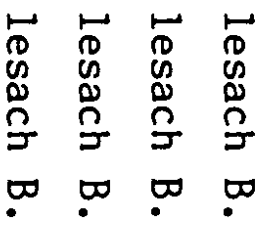


Als Ergebnis stellen wir vorläufig fest:

1. Innerhalb des funktionalen Paradigmas (Kovtunova) hat die $\mathrm{T}-\mathrm{C}-\mathrm{A}$ von $\mathrm{Th}_{3} / \mathrm{Rh}_{3}$ der kontextfreien Form ihre Entsprechung in der kontextabhängigen Form (ebenfalls $\mathrm{Th}_{3} / \mathrm{Rh}_{3}$ ). Ebenso entsprechen sich T-C-A von $\mathrm{Th}_{5} / \mathrm{Rh}_{5}\left(\mathrm{~S}_{1 \mathrm{KS}}\right)^{\text {und }} \mathrm{Th}_{1} / \mathrm{Rh}_{1}\left(\mathrm{~S}_{1 \mathrm{KF}}\right.$ ).

2. Erwartungsgemä $B$ - da jetzt in der Position des Thema entfällt $\mathrm{Th}_{2} / \mathrm{Rh}_{2}$ bei $\mathrm{S}_{1 \mathrm{KS}}{ }^{\circ}$

3. Th ${ }_{6} / \mathrm{Rh}$ und $\mathrm{Th}_{7} / \mathrm{Rh}_{7}$ sind $\mathrm{T}-\mathrm{C}-\mathrm{A}$ von $\mathrm{S}_{\mathrm{KS}}$, für die $\mathrm{S}_{1 \mathrm{KF}}$ keine Entsprechungen aufweist.

Bevor wir die Ergebnisse kommentieren werden, und sich die Untersuchung im besonderen den Sätzen mit $\mathrm{Th}_{6} / \mathrm{Rh}_{6}$ und $\mathrm{Th}_{7} / \mathrm{Rh}$ zuwenden wird, sollen zunächst die erhaltenen Ergebnisse 1-3 an weiteren Beispielsätzen überprüft werden. Dabei kann $Q_{5}$ unberücksichtigt bleiben, da von dieser $T-C-A$ von S 1 KS' bei der allein die Verbergänzung Thema ist, keine direkten Aufschlüsse für unsere Fragestellung $z u$ erwarten sind. Darüberhinaus ist ihre Realisierung aufgrund der Regeln, die aus der Rektion der Verben resultieren, nur bedingt möglich. 


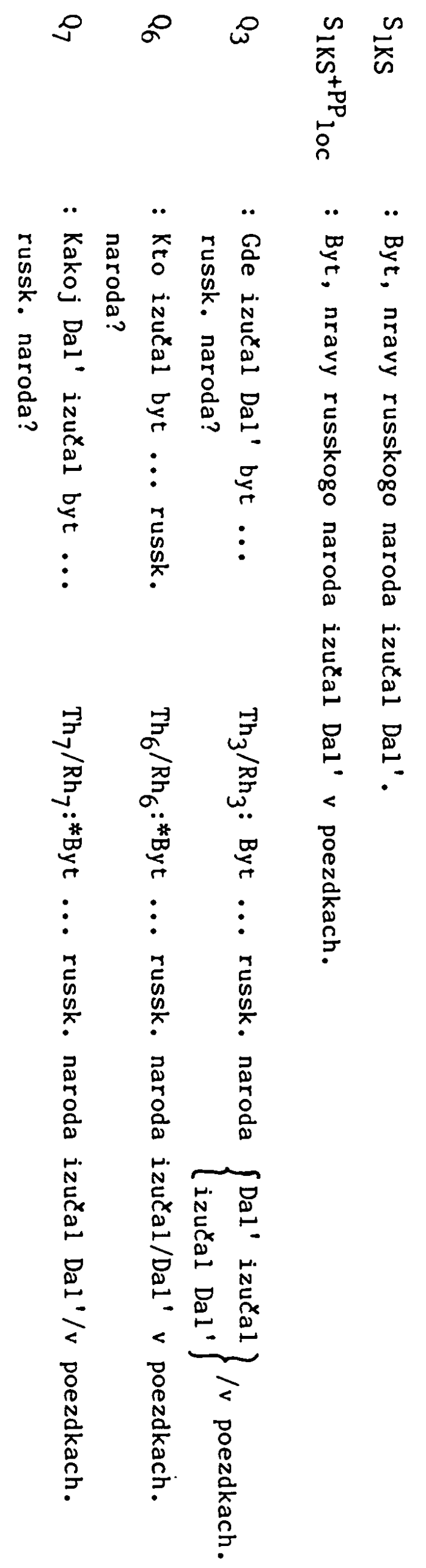

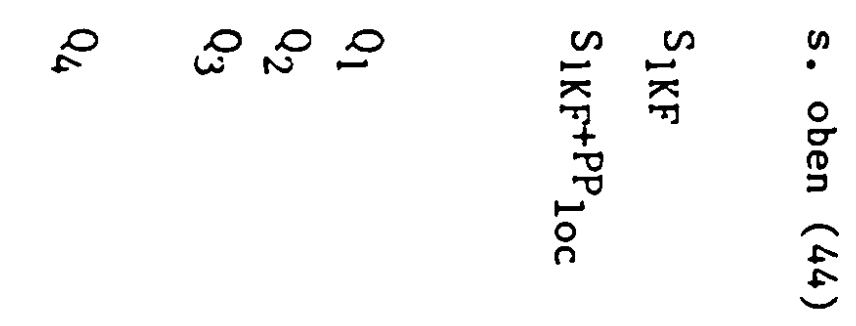

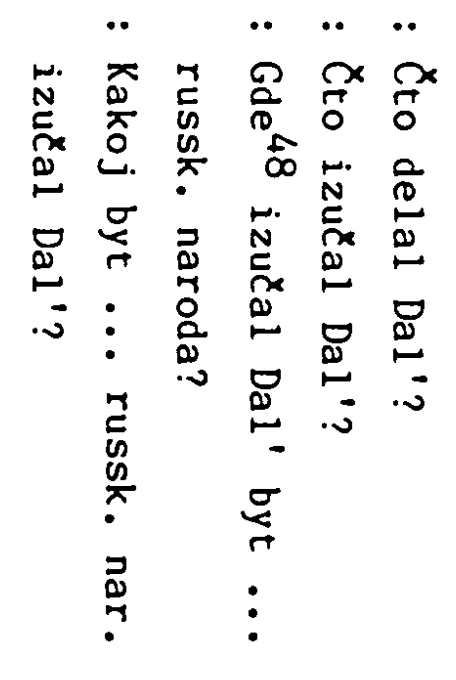

$\stackrel{\mathscr{D}}{\stackrel{\mathscr{N}}{*}}$

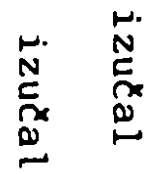

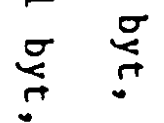

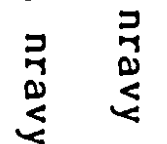

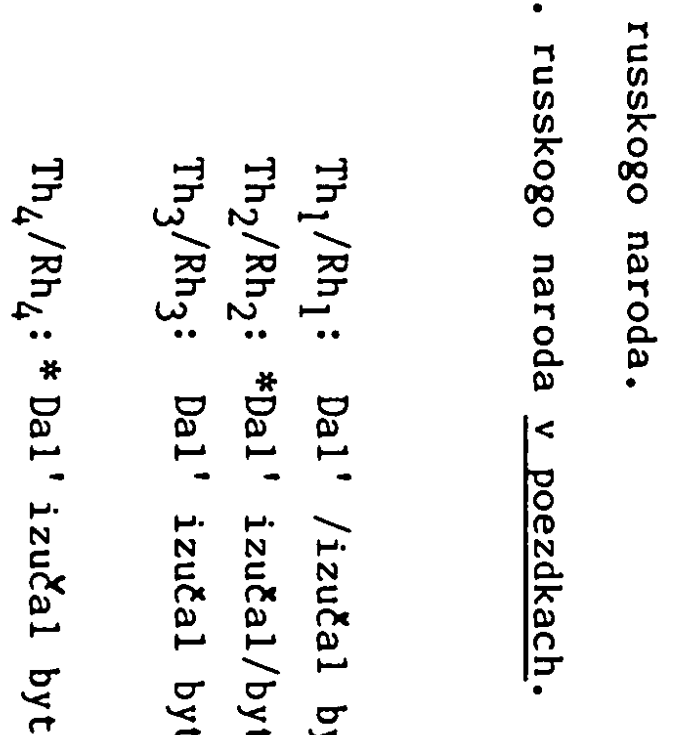

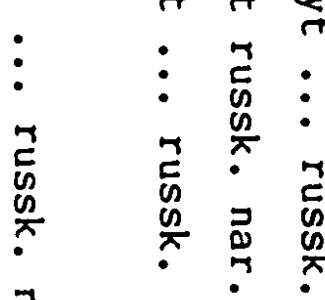

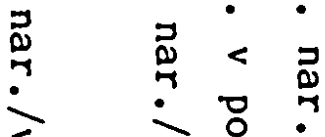

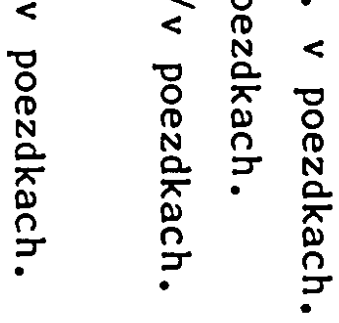


Deti/smotreli na koßku $\mathbf{v}$ sadu.

Deti smotreli/na koßku $v$ sadu. Deti smotreli na kosku/v sadu. Deti smotreli na kosku/v sadu.

Na kosku $\left\{\begin{array}{l}\operatorname{det} i \text { smotreli } \\ \text { smotreli deti }\end{array}\right\} / v$ sadu.

Na košku smotreli/deti $v$ sadu.

$\mathrm{Na}$ košku smotreli deti/v sadu. 


\section{(109)}

$S_{1 K F} \quad$ : Deti smotreli na kosku.

$\mathrm{S}_{1 K \mathrm{~F}}+\mathrm{PP} 1 \mathrm{loc}$ : Deti smotreli na koSku v sadu.

$Q_{1}$

$\mathrm{Q}_{2}$

$\mathrm{Q}_{3}$

$\mathrm{Q}_{4}$

$\mathrm{S}_{1 \mathrm{KS}}$

$\mathrm{S}_{1 K S}+{ }^{P P_{10 c}}$ : Na koßku smotreli deti $\mathrm{v}$ sadu.

$\mathrm{Q}_{3}$

$Q_{6}$

$Q_{7}$
: Cto delali deti?

: Na cto smotreli deti?

: Gde smotreli deti na kosku?

: Na kakuju koß̌ku smotreli deti?

: Na kosku smotreli deti.

: Gde na koskku smotreli deti?

$\mathrm{Th}_{3} / \mathrm{Rh}_{3}$ :

$\mathrm{Th}_{6} / \mathrm{Rh}_{6}:$

: Kakie deti smotreli na kołkku?
$\mathrm{Th}_{1} / \mathrm{Rh}_{1}:$

$\mathrm{Th}_{2} / \mathrm{Rh}_{2}$ :

$\mathrm{Th}_{3} / \mathrm{Rh}_{3}$ :

$\mathrm{Th}_{4} / \mathrm{Rh}_{4}:$ 
Eine Gegenüberstellung der Ergebnisse der T-C-A in der kontextfreien und der kontextabhängigen form von (44) und (109) bestätigt und verdeutlicht die Ergebnisse, die wir zu (25) erhalten hatten:

- $\mathrm{Th}_{3} / \mathrm{Rh}_{3}$, d.h. die $\mathrm{T}-\mathrm{C}-\mathrm{A}$, bei der VP durch das Lokaladverbial näher bestimmt wird, ist in $S_{1 K F}$ und $S_{1 K S}$ entsprechend möglich.

- Th $6_{6} / \mathrm{Rh}$ und $\mathrm{Th}_{7} / \mathrm{Rh}$ gehen auf Sätze zurück, die entweder nicht akzeptabel sind (44) oder solche T-C-A aufweisen, die in dem kontextfreien Ausgangssatz keine Entsprechungen haben (25, 109), d.h. sie gehören $z u$ einem anderen funktionalen Paradigma.

- $\mathrm{Th}_{2} / \mathrm{Rh}_{2}$ ist an die Bedingung gebunden, daß die verbergänzung im betreffenden Oberflächensatz durch die PP näher bestimnt werden kann. Das ist nicht der Fall bei (44). Welche Aufschlüsse geben uns diese Ergebnisse über die Prädikationsmöglichkeiten und -beschränkungen von PP loc unter funktionalem Aspekt?

Eine Gegenüberstellung der Ergebnisse, die einerseits der Prädikations-Test (s. Kap. 3.1.1 besonders Schema 1) und andererseits der Thema-Rhema-Gliederungstest liefert, ergibt Folgendes:

1. Die Ergebnisse beider Tests entsprechen sich insoweit, als PP loc Rhema proprium der Aussage sein kann, d.h. der Fall vorliegt, daß alle Satzglieder links von PP loc zum Thema gehören.

In diesen Fällen kann PP lọc eine nähere Bestimmung zum VPKomplex sein ' (s. $\left.Q_{3}\right)$ und nähere Bestimmung zur Verbergänzung (s. $Q_{4}$ ). Die tiefenstrukturelle Mehrdeutigkeit findet in der funktionalen eine gewisse Entsprechung.

2. Die Ergebnisse der beiden Testsorten decken sich jedoch insoweit nicht, als nach dem Thema-Rhema-Gliederungs-Test die PP $10 \mathrm{C}$ in Endstellung nicht eine Aussage über NP, soweit es Thema ist, macht. Tiefenstrukturell gesprochen heibt das, daß die PP loc nicht über NP prädiziert werden kann, jedenfalls nicht direkt, sondern nur indirekt, insofern sie - wie 
bereits ausgeführt - über VP prädiziert werden kann und diese ihrerseits in prädikativer Relation $\mathrm{zu}^{\mathrm{NP}}{ }^{1}$ steht. Wenn dieser Schluß in Hinblick auf die kontextfreien sätze noch nicht ausreichend eviderıt ist, so können die letzten Zweifel hierüber bei der Analyse der kontextabhängigen Sätze ausgeräumt werden.

Mit der Anwendung des Thema-Rhema-Gliederungs-Tests auf die folgenden Beispiele wollen wir uns auf diese Frage konzentrieren: stimmt es, daß die tiefenstrukturell mögliche Prädikation der PP ${ }_{\text {loc }}$ über NP funktional blockiert ist? Wenn $\mathrm{ja}$, in welchem Kontext der funktionalen Gliederung ist diese Restriktion $z u$ sehen? Es werden im folgenden kontextfreie und kontextabhängige Satzpaare einander gegenübergestellt. Das zeichen "/" bedeutet mögliche Grenze( $n)$ von Thema und Rhema; " $Q$ " verweist auf die vorauszusetzende(n) $\operatorname{Frage}(n)$ :

( 42 ) Gost' / kupil knigu / $v$ nasem kioske.

( 42a) knigu $\left\{\begin{array}{l}\text { gost' kupil } \\ \text { kupil gost' }\end{array}\right\}^{49} / v$ našem kioske.

$Q_{\frac{1}{2}} Q_{3}$

( 46 ) Ja / Cital doklad / v Russk. Botan. obšcestve.

$Q_{1} \cdot Q_{3}$

( 46a) Doklad $\left\{\begin{array}{l}j a \text { ¿ital } \\ \text { ¿tal ja }\end{array}\right\} / v$ Russk. Botan.

$Q_{3}$ obšcestve.

( 48 ) L.I. Brežnev / podtverdil èti principy / na Berlinskoj konferencii.

( 48a) Éti principy $\left\{\begin{array}{l}\text { L.I. Brežnev podtverdil } \\ \text { podtverdil L.I. Brežnev }\end{array}\right\} / Q_{3}$ na Berlinskoj konferencii.

(110) Roditeli / zdali detej / na vokzale.

(110a) Detej $\left\{\begin{array}{l}\text { roditeli zdali } \\ \text { zdali roditeli }\end{array}\right\}$ / na vokzale.

$Q_{1} \cdot Q_{3}$

(4) Pionery / videli pervogo sekretarja obkoma / na stroitel'noj plostadke.

( 4a) Pervogo sekretarja obkoma pionery videli / na stroitel'noj plošadke. 
4b) Pervogo sekretarja obkoma videli / pionery / na stroitel'noj plošcadke.

( 25 ) Pamjatniki / napominajut o partizanach / v selach i lesach Belorussii.

( 25a) O partizanach pamjatniki napominajut / v selach i lesach Belorussii.

( 25b) O partizanach napominajut / pamjatniki / v selach i lesach Belorussii.

( 26 ) Knjaz' / proizvel perevorot / v Mordasove.

( 26a) Perevorot knjaz' proizvel / v Mordasove.

( 26b) Perevorot proizvel / knjaz' / v Mordasove.

(109) Deti / smotreli na košku / v sadu. (109a) Na koßkku deti smotreli / v sadu. (109b) Na koß̌ku smotreli /deti / v sadu.
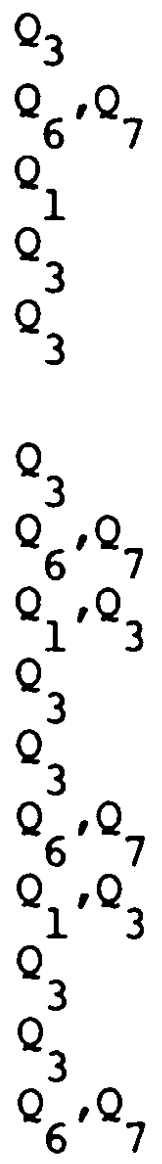

Die Wahlmöglichkeiten in der Wortfolge innerhalb des Thema in den kontextabhängigen Sätzen, die in den Beisp. $(42,46,48$, 110) für unsere Frage irrelevant sind, sind dagegen in den Beispielen (4, 25, 26, 109) aufschlubreich: während jeweils (a) nur eine kontextabhängige Entsprechung des kontextfreien Satzes ist - wobei erwartungsgemä $B$ in (a) die funktionale Mehrdeutigkeit aufgehoben ist - hat (b) Lesarten, die nicht nur als Antwort auf $Q_{3}$ verstanden werden, sondern andere Bedeutungen des Satzes implizieren $\left(Q_{6}, Q_{7}\right)$. $Q_{6}$ setzt eine $T-$ $C-A$ voraus, die bei den Beispielen $(42,44,46,48,110) 2 \mathrm{u}$ unsinnigen Sätzen führt, man vgl.:

(44b) *Byt ... russ, naroda izučal / Dal' v poezdkach.

Die beiden - wie es zunächst scheinen mag - widersprüchlichen Ergebnisse: $Q_{6}$ gründet bei einer Menge von kontextabhängigen Sätzen auf Lesarten, die in den entsprechenden kontextfreien Sätzen nicht enthalten sind; bei einer anderen Menge von Sätzen kann $Q_{6}$ nur als unsinnige Frage gewertet werden - 
diese scheinbar widersprüchlichen Ergebnisse sind durch einen gemeinsamen Faktor bedingt: $Q_{6}$ gliedert die Satzglieder im Rahmen des Thema und damit auch im Rahmen des Rhema in einer Weise, die keiner Gliederungsmöglichkeit in den entsprechenden kontextfreien Sätzen entspricht. Das geschieht dadurch, daB eine direkte Kontaktstelle ${ }^{51}$ von PP ${ }_{l o c}$ und NP gebildet wird: "pionery na stroitel'noj plošadke" - was für die funktionale Analyse bedeutet, daß dieses Syntagma in ein und demselben Bereich stehen kann - in dem des Thema oder in dem des Rhema. Damit steht dieses Ergebnis im Widerspruch sowohl zu $Q_{1}$, als auch zu $Q_{3}$. Kontextabhängige Sätze, die Antworten auf $Q_{6}$ und $Q_{7}$ sind, gehen auf kontextfreie sätze mit anderer Struktur zurück; sie haben folgende Oberflächenrealisierung:

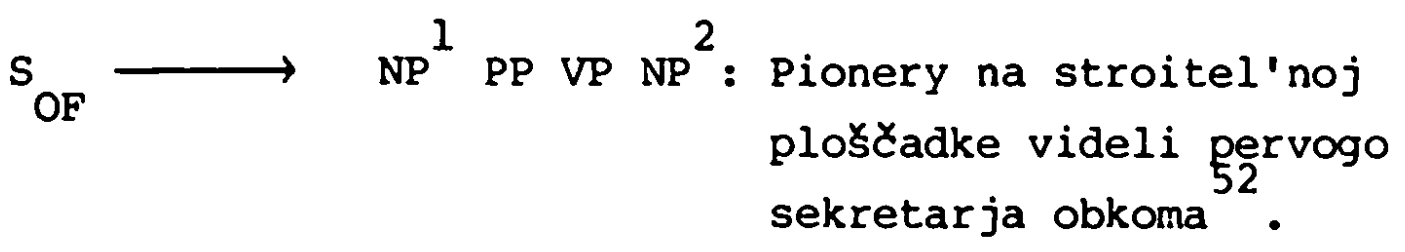

Bei dieser struktur findet andererseits die tiefenstrukturelle Analyse ihre Bestätigung, daß nämlich die PP über NP prädiziert werden kann.

Die in (4b) etc. dargestellte Mehrdeutigkeit ist nicht auf kontextabhängige Sätze beschränkt, sondern es muß mit ihr dann gerechnet werden, wenn PP Rhema proprium ist und die semantischen Bedingungen einer Prädikation erfüllt sind. So kann z.B. (25) mit folgender T-C-A: "Pamjatniki napominajut o partizanach / $v$ selach $i$ lesach Belorussiin, als Aussage über Ausstellungsstücke in einem Museum in Kiev verwendet werden ("O kakich partizanach napominajut èti pamjatniki?"), aber auch als Aussage über Mahnmale in WeiBrubland ("Gde napominajut pamjatniki o partizanach?").

Der beschriebenen Mehrdeutigkeit kann unter Umständen durch die kontextabhängige Form der Aussage entgangen werden; man vgl.: 
(111) Ob ètom knjaze / vsegda rasskazyvalis' trezvy Cajno strannye vesti $\mathrm{v}$ Mordasove.

Die kontextfreie Form ist - wie (25) - funktional und syntaktisch mehrdeutig:

(112) Crezvy̌ajno strannye vešxi vsegda rasskazyvalis' o knjaze $\mathrm{v}$ Mordasove.

In (112) kann - im Unterschied zu (111) - die PP loc zum einen nähere Bestimmung $z u$ VP sein, zum anderen zur verbergänzung.

Unsere Frage: wirkt der funktionale Faktor disambiguierend in Sätzen mit PP in Endstellung, läßt sich mit Hilfe des Thema-Rhema-Gliederungs-Tests positiv beantworten: die Analysen der kontextfreien und der entsprechenden kontextabhängigen Sätze zeigen, daß die Prädikationsmöglichkeiten von PP $10 c$ auf den funktionalen Bereich beschränkt sind, in dem sie steht; d.h. für PP 10 in Endstellung auf den Bereich des Rhema. Ist PP loc Rhema proprium, so kann der satz funktional und grammatisch mehrdeutig sein; jedoch die Prädikation durch $\mathrm{PP}{ }_{10 \mathrm{C}}$ ist auf die VP beschränkt: sie ist nähere Bestimmung zum finiten verb und/oder zum objekt. Das sprachliche Kriterium für zulässige Prädikationen bei der Bildung von Sätzen ist damit die Grenze von Thema und Rhema. Wird diese Grenze "verletzt", so erhalten wir entweder unsinnige Sätze (44b etc.) oder Sätze mit einer anderen Strukturierung der Aussage, womit impliziert ist - mit einer anderen Bedeutung ( $4 b$, etc.).

Aus den Analyseergebnissen lassen sich folgende Regeln ableiten:

1. für kontextfreie Sätze: die tiefenstrukturell mögliche Prädikation der PP über NP bei Endstellung des Adverbials ist durch den funktionalen Faktor der "Grenze" von Thema und Rhema für die Aussagegliederung blockiert.

Daraus ergibt sich 2. für die den kontextfreien entsprechenden kontextabhängigen Sätze: in diesen ist die Wortfolge blockiert, die zu der Bildung einer Kontaktstelle 
von PP 1 loc und $N P^{l}$ führt.

Sätze von TYp (4b) und (44b) geben noch weitere Aufschlüsse über strukturelle Eigenschaften von Sätzen mit ${ }_{10}{ }_{10 c}$ in Endstellung:

1. Die beschriebene Mehrdeutigkẹit von Sätzen wie (4b), nämlich, daß sie funktionalen Paradigmen zweier verschiedener Oberflächensätze mit folgenden strukturen angehören können:

$\begin{array}{llllll}\mathrm{S}_{1 \mathrm{KF}} \longrightarrow \mathrm{NP}^{1} & \mathrm{VP} & \mathrm{NP}^{2} & \mathrm{PP}_{2} & \text { und } \\ \mathrm{S}_{2 \mathrm{KF}} & \longrightarrow & \mathrm{NP}^{1} & \mathrm{PP} & \mathrm{VP} & \mathrm{NP}^{2}\end{array}$

deutet auf eine bestimmte Hierarchie der funktionalen und syntaktischen Faktoren bei der Ableitung dieser Sätze hin: wechseln Satzglieder, über die durch PP ${ }_{10 c}$ prädiziert wird, ihre position von Thema zu Rhema oder umgekehrt, so mus die PP mitwechseln, andernfalls werden die syntaktischen Beziehungen der PP ${ }_{\text {loc }}$ zu ihren Argumenten aufgehoben, oder es entstehen syntaktische Beziehungen anderer Art, die ihrerseits Sätze mit anderer Bedeutung zur Folge haben. Aus diesen Beobachtungen läßt sich ein Modell für die Erweiterung von Sätzen mit PP nach der Gliederung der Aussage in ihrer pradigmatisch starken Form erfolgt.

2. folgt daraus, daß für eine Modellierung der Ableitung von Sätzen mit PP die Wahl des Arguments für die lokale Prädikation auf loc iner Stufe vor der wahl der aktuellen Satzperspektive in ihrer Abhängigkeit vom Kontext erfolgt. Es muß auf die paradigmatisch starke form der Aussage zurückgegangen werden, da sich auf ihre Grenzen von Thema und

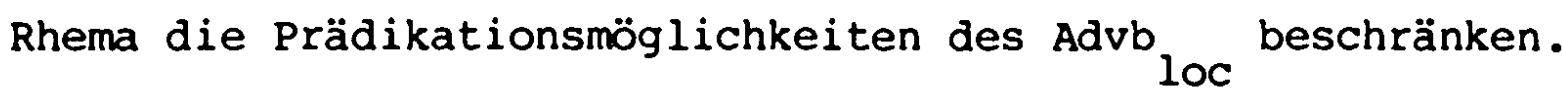


In dem folgenden Schema einer Modellierung der Ableitung von Sätzen mit PP ${ }_{\text {loc }}$ in Endstellung werden die Ergebnisse der tiefenstrukturellen und der funktionalen Analysen zusammengefast:

Schema 2:

funktionale Ebene der Ableitung

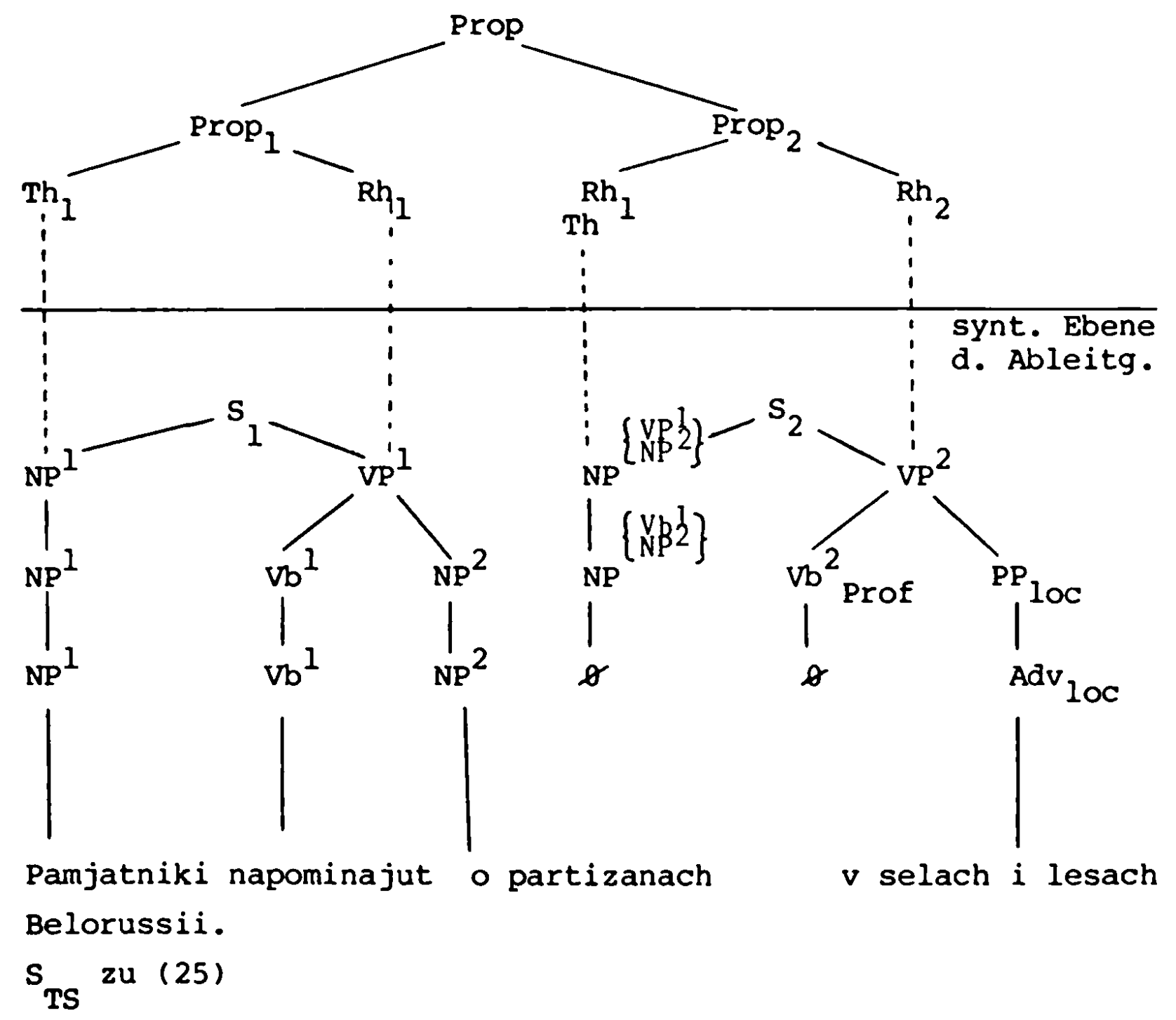


Statistische Untersuchungen (Krzizkova, 1974, 47) zeigen, das Endstellung der PP loc auffallend in Beispielen der Satzstruktur:

$\mathrm{S} \longrightarrow$ NP VP PP ${ }_{\text {loc }}$ verbreitet ist. Die Ergebnisse des von mir durchgearbeiteten Corpus entsprechen dieser Beobachtung. Der Frage, in welchem Erklärungszusammenhang diese verbreitete Verwendung der $\mathrm{PP}$ loc in Sätzen mit der struktur $\mathrm{S} \longrightarrow$ NP VP zu sehen ist, wird im Zusammenhang mit der Darstellung von $\mathrm{PP}$ loc in Initialstellung wieder aufgegriffen (s.u. S. 117). Hier sollen vorläufig die bisherigen Analyseergebnisse $2 \mathrm{u} P \mathrm{PP}$ in Endstellung an Beispielen der Satzstruktur $S \longrightarrow \quad N P{ }^{10 C}{ }^{P P}{ }_{l O C}$ überprüft werden. .

Die Ergebnisse $z u$ der Frage nach den tiefenstrukturellen Prädikationsmöglichkeiten der PP führten $z u$ der Frage nach restriktiven Faktoren für die Prä́dikation - von den möglichen Faktoren stand dann der funktionale im Mittelpunkt der Untersuchung, die ergab, daß die Grenze zwischen Thema und Rhema ein solcher Faktor ist, und zwar in dem Sinn, das die Erweiterungsmöglichkeit von Satzgliedern durch PP Wortstellungsregeln impliziert. Aus den Ergebnissen $z u$ dec Sätzen (4) etc. und (44) etc. lieb sich die Regel ableiten, das die PP ${ }_{10 C}$ in demselben Bereich der aktuellen Gliederung stehen mus - Thema oder (vel) Rhema - wie das Satzglied, über das sie ausgesagt wird. zu diesem Problemkreis geben die Sätze der struktur $S \longrightarrow N P$ VP PP keine neuen Erkenntnisse, andererseits findet die bisherige Analyse - auf sie angewendet - ihre volle Bestätigung.

Die folgende Anwendung des Thema-Rhema-Gliederungs-Tests dient der Uberprüfung des obigen Ergebnisses: die Prädikation der PP 10 über NP ist blockiert bzw. eine solche Prädikation ergibt einen anderen Satz.

Man vgl. folgende Beispiele: 
( 15 ) Pervyj sekretar' / vystupaet / na sovesc. partijno-chozj. aktiva

Kazachstana.

( 15a) ... vystupaet pervyj sekretar' /

na soveš. partijno-chozj. aktiva

$Q_{3}$

Kazachstana.

( 15b) *... vystupaet / pervyj sekretar'/ na soveščanii ...

( 19 ) Zvezdy / zažigalis' / na rozovom nebe.

( 19a) Zazigalis' zvezdy / na rozovom nebe.

( 19b) *Zažigalis' / zvezdy / na rozovom nebe.

(113 ) Gosti / obedali / na balkone.

(113a) Obedali gosti / na balkone.

$(113 b)(*)$ Obedali / gosti / na balkone.

( I ) Vor / isčez / v tolpe ljudej.

( la) Istez vor / $v$ tolpe ljudej.

( Ib) *Istez / vor / $v$ tolpe ljudej.

(17) Malxik / spal / v komnate storoza vodokacki.

( 17a) Spal mal'tik / v komnate storoža vodokacki.

( 17b) Spal / mal'Łik / v komnate storoza vodokacki.

( 65 ) Gosti / gromko razgovarivali / v perednej.

( 65a) Gromko razgovarivali gosti / $v$ perednej.

( 65b) Gromko razgovarivali / gosti / v perednej.

$Q_{6}, Q_{7}$

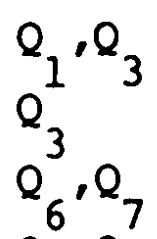

$Q_{1}, Q_{3}$

$Q_{3}$

$\mathrm{Q}_{6}, \mathrm{Q}_{7}$

$Q_{1}, Q_{3}$

$Q_{3}$

$Q_{6}^{3} \cdot Q_{7}$

$Q_{1}, Q_{3}$

$\mathrm{Q}_{3}$

$Q_{6}, Q_{7}$

$Q_{1} \cdot Q_{3}$
$Q_{3}$
$Q_{6} \cdot Q_{7}$

Der Thema-Rhema-Gliederungs-Test, angewendet auf Sätze der Struktur S $\longrightarrow$ NP VP PP ${ }^{1}$, ergibt wie oben zwei Mengen von Sätzen: $(1,15,19,113)^{\circ}$ weisen in kontextfreien Sätzen zwei mögliche T-C-A auf: PP ${ }_{\text {loc }}$ ist Rhema proprium oder einen nähere Bestimmung $2 \mathrm{u}$ VP.

Auch (17, 65) entsprechen insoweit $(1,15,19,113)$. Die Bildung kontextabhängiger Sätze dieser beiden Mengen weist dagegen Unterschiede auf; während für $(1,15,19,113)$ nur eine sinnvolle Gliederungsmöglichkeit der T-C-A zulässig (15a etc.) ist, sind bei $(17,65)$ NP und $\mathrm{PP}$ loc lexikalisch so besetzt, daB darüberhinaus eine weitere kontextabhängige Form 
(vgl. 17b, 65b) gebildet werden kann, die eine semantische Beziehung und damit eine Prädikation der PP loc über NP enthält. Diese Lesart geht auf folgende kontextfreie Form der Aussagegliederung zurück:

$\mathrm{S}_{\mathrm{OF}} \longrightarrow \mathrm{NP}^{\mathrm{l}}$ PP VP, d.h. auf Sätze mit einer anderen Bedeutung, Sätze, die zu einem anderen funktionalen Paradigma gehören. Auch hier wirkt die Grenze von Thema und Rhema restriktiv. Der Test bestätigt damit die Regel, daß die tiefenstrukturell mögliche Prädikation der PP ${ }_{10 c}$ über $\mathrm{NP}^{1}$ bei Finalstellung der ${ }^{P P}{ }_{l o c}$ aufgrund funktionaler Faktoren blockiert ist. 
3.2.2 SÄTZE MIT PP ${ }_{1 O C}$ VOR UND NACH DEM FINITEN VERB

3.2.2.1 SÄTZE MIT PP ${ }_{\text {IOC VOR DEM FINITEN VERB }}$

Die Untersuchungen zu PP tive Regeln für die Prädikationsmöglichkeiten durch das Lokaladverbial. Diese Regeln sind bedingt durch die Grenze von Thema und Rhema: die Prädikationsmöglichkeiten beschränken sich auf Argumente im Bereich des Rhema, insoweit die PP nicht Rhema proprium ist; für diesen Fall ergeben sich Prầdikationsmöglichkeiten, die sich auf die verbalphrase im ganzen beziehen und/oder nur auf die Verbergänzung. Für die Untersuchungen wurde die paradigmatisch starke Form der Aussage zugrunde gelegt.

Die Analysen $z u(4)$ und (7) etc., im besonderen ( $4 b$ ) etc., deuten darauf hin, daß Sätze mit stellung der PP ${ }_{\text {loc }}$ vor dem finiten verb eine weitere interessante subklasse ${ }_{\text {innerhalb }}$ der Sätze mit PP bilden, deren besondere Merkmale ebenfalls von der Grenze zwischen Thema und Rhema in der aktuellen Gliederung bedingt sind. Die Analysen der kontextabhängigen Sätze mit PP ${ }_{\text {loc }}$ in der Endstellung machen darauf aufmerksam, daß S $S_{\text {KS }}$ mit den T-C-A: $T_{6} / \mathrm{Rh}_{6}$ und $\mathrm{Th}_{7} / \mathrm{Rh}, \mathrm{zu}$ dem funktionalen Paradigma gehören, das die folgende kontextfreie Form hat:

$\mathrm{S}_{\mathrm{KF}} \longrightarrow \mathrm{NP}^{\mathrm{I}} \mathrm{PP}_{\mathrm{lOC}} \mathrm{VP}\left(\mathrm{NP}^{2}\right)$

vgl. (3) Vor $v$ tolpe ljudej isčez.

(6) Pionery na stroitel'noj plošadke videli pervogo sekretarja obkoma.

Eine erste Antwort auf die Frage nach der Funktion der PP vor dem-finiten Verb ist damit folgende: es ist diese Position, in der die PP ${ }_{l o c}{ }^{N}$ näher bestimmt, d.h. 
tiefenstrukturell liegt hier die Prädikation von $\mathrm{PP}_{\text {loc }}$ über $\mathrm{NP}^{\mathrm{I}}$ vor und funktional haben wir folgende $\mathrm{T}-\mathrm{C}-\mathrm{A}$ :

(3a) $\mathrm{Th}_{1} / \mathrm{Rh}_{1}$ : Vor $\mathrm{v}$ tolpe ljudej / isčez. ${ }^{54}$ $Q_{1}{ }^{1}$ : Cto bylo s vorom $v$ tolpe 1 judej?

Jedoch berücksichtigt diese Antwort nicht, daß (3) auch mit folgender T-C-A verstanden werden kann:

(3b) $\mathrm{Th}_{1} / \mathrm{Rh}_{1}$ : Vor / $\mathrm{v}$ tolpe ljudej isčez.

$Q_{1}^{1}{ }^{1}$ : Cto sdelal vor?

Folgende Beispiele sollen die damit angesprochenen verschiedenen Funktionen der PP loc vor dem finiten verb verdeutlichen. Der Thema-Rhema-Gliederungs-Test wird in verkürzter Form angewendet; "/" markiert die angenommene( $n)$ Grenze( $n)$ von Thema und Rhema:

(114) Takoe koliCestvo chleba / $\underline{v}$ oblasti zagotovleno vpervye.

(115) Mar'ja Dmitrievna / $\mathrm{v}$ duše požalela o svoem chorosen'kom Pokrovskom s veseloj rečkoj.

(116) Trudovoj den' / na prostorach strany ne končaetsja.

(117) Liza .... vsemu v dome / poklonilas' v poslednij raz.

(118) List'ja na derevjach / stali želtymi.

(119) Serdce (/) v Lavreckom / drognulo ot Zalosti i ljubvi.

(120) (V serdce buševal ogon' ...) Plamja (/) v grudi / razgoralos' vse jarte.

(121) V sootvetstvii s programmoj sotrudni Xestva meždu Sovetskim Sojuzom i Franciej / na stancii "Saljut-6" / zaplanirovan rjad sovmestnych èsperimentov po kosmi ̌eskomu materialovedeniju.

In den obigen Sätzen steht die PP zwischen einer nominalen Phrase, die auch eine oblique (110C) oder eine Präpositionalphrase (121) sein kann, und dem finiten Prädikat, das 
verschiedene Ergänzungen haben kann. Fragen wir zunächst noch ohne formalen Rahmen nach den Beziehungen der PP zu den übrigen Satzgliedern, so erhalten wir folgende unterschiedliche Antworten: in (114), (115) und (116) bestimmt die PP das finite Verb näher, in (117) und (118) die nominale Phrase; in (119) versteht der native-speaker-Informant spontan die PP loc als nähere Bestimmung zu NP, läBt jedoch als mögliche Beziehung der $P P$ auch die zum finiten Verb gelten. Der Kontext hebt in $(120)$ die mögliche Mehrdeutigkeit der Beziehung von "v grudi" auf: "Plamja $v$ grudi" nimmt die Aussage des vorangehenden Satzes "v serdce buseval ogon'", auf und ist damit Thema. In einem anderen Kontext könnte " $v$ grudi" nähere Bestimmung zum finiten Verb sein. In (121) ist die Beziehung ambig: der Satz kann in dem Sinn verstanden werden, daß die PP die nominale Phrase näher bestimmt, wie auch in dem Sinn, daß sie das finite Verb näher bestimmt; (s. dazu Näheres unten S. 111).

Im Rahmen unseres 2-Phasen-Beschreibungsmodells lassen sich die unterschiedlichen und mehrdeutigen Beziehungsmöglichkeiten der PP ${ }_{\text {loc }}$ einordnen und beschreiben. Für die jeweilige Funktion der ${ }_{\mathrm{PP}}^{\mathrm{loc}}$ in den zur Diskussion stehenden Sätzen ist wiederum die Grenze von Thema und Rhema bestimmend. In der Position der PP ${ }_{\text {loc }}$ vor dem finiten Verb kann die Grenze - je nach Absicht des Sprechers oder nach Aufbau des Textes - so variieren, daß die PP entweder zum Rhema oder zum Thema gehört. $\mathrm{zu}$ dieser funktionalen Mehrdeutigkeit kommt, bedingt durch Faktoren, die in der tiefenstrukturellen Analyse untersucht wurden, die grammatische. Man muß daher feststellen, daß die PP 10 in der Position vor dem finiten verb im besonderen in der paradigmatisch starken Form der Aussage potentiell $^{55}$ mehrdeutig ist. Hier sind es Faktoren wie "Vorwissen" und Kontext, die diese Ambiguität aufheben können.

Der Faktor "Vorwissen" kann relevant sein, wenn semantisch die Aussage mit PP ${ }_{\text {loc }}$ u einem der tiefenstrukturell möglichen Argumente nicht past oder banal ist; daraus folgt das 
"spontane" Verständnis der Beziehung der PP ${ }_{10 c}$ in (115) auf das finite Verb, in (118) auf $\mathrm{NP}^{1}$.

In (114) "verträgt" sich die Prädikation "v oblasti" nicht mit der durch den Quantor "takoe količestvo" näher bestimmte NP "chleb". Vgl. dagegen:

(123) Chleb / v oblasti / vpervye zagotovlen novymi kombajnami.

Der Kontext wirkt disambiguierend in:

(124) Odnako pri dostatołnosti trudovych resursov po strane $v$ celom / sušestvujut znatitel'nye territorial'nye disproporcii mezdu potrebnost'ju $v$ rabočj sile $i$ ee nalixiem.

Aufgrund der kontextuellen Gegenüberstellung "po strane v celom" und "territorial'nyj" versteht man in (124) die PP loc als nähere Bestimung zu "resursy"; vgl. dagegen:

(125) Odnako pri dostatoknosti trudovych resursov / po strane $\mathrm{v}$ celom / sušcestvujut značitel'nye disproporcii meždu potrebnost'ju $v$ rabołej sile $i$ ee nalitiem.

(119) erinnert an die Beobachtungen $z u(81)$, die darauf aufmerksam machten, daß oberflächenstrukturell die semantischen Unterschiede von präpositionaler und attributiver Ergänzung zusammenzufallen scheinen; so können wir anstelle von (114) konstruieren:

(126) Serdce Lavreckogo drognulo ot zalosti i ljubvi.

In diesem zusammenhang findet die Beobachtung ihre systematische Erklärung, daß PP ${ }_{\text {loc }}$ in der stellung vor dem finiten Verb und Relativsatz ${ }_{56}$ syntaktisch synonyme oberflächenstrukturen sein können. Man vgl.: 
(127) Listja, kotorye (visjat) na derevjach, stali Zeltymi.

(128) Vor, kotoryj byl $v$ tolpe ljudej, isčez.

(129) Pionery, kotorye byli na stroitel'noj plošcadke, videli pervogo sekretarja.

(130) Vsemu, ¿̌to est' $v$ dome, Liza poklonilas' ...

Analysiert man die PP ${ }_{\text {loc }}$ in dem hier vorgeschlagenen ZweiPhasen-Modell, so läßt auch der Faktor "Kontext", der sich in linguistischen Arbeiten mit formalisierten Beschreibungsmethoden weitgehend einer Analyse entzieht, Regelmäßigkeiten, die der Disambiguierung der Funktionen von PP loc dienen, erkennen. Dazu folgendes Beispiel:

(131) Ėkspansivnyj Magomed Ataev ot svoej podpisi v priemnosdatoknych dokumentach / nemedlenno otkazalsja.

Rhema der - syntagmatisch starken - Aussage ist "nemedlenno otkazalsja". Die PP steht im Bereich des Thema, ist hier nähere Bestimmung $z$ lochodpis'". In der syntagmatisch starken Form der Aussage ist die aktuelle Gliederung eindeutig, die daraus resultierende Wortfolge führt zu ebenfalls eindeutigen syntaktischen Beziehungen des lokalen Prädikats zu seinem Argument.

Bilden wir die paradigmatisch starke Form der Aussage mit der Stellung der PP ${ }_{10 c}$ vor dem finiten Verb, erhalten wir einen Satz, der funktional und syntaktisch mehrdeutig ist:

(131a) Ėkspansivnyj Magomed / v priemno-sdatoßnych dokumentach nemdlenno otkazalsja / ot svoej podpisi.

Die PP 10 ist in (13la) nähere Bestimmung zum finiten Verb; wo die besagte "Unterschrift" geleistet wurde, ist bei der stellung der PP $10 \mathrm{cor}$ dem finiten verb, wie aber auch nach dem finiten verb, vgl.: 

(13lb) ... / nemedlenno otkazalsja $v$ priemno-sdatocnych dokumentach / ot svoej podpisi.

nicht explizit genannt.

Für eine Menge von Sätzen ist es "nur" das Hintergrundswissen zu dem Kontext, das dessen funktionale und syntaktische Mehrdeutigkeit aufhebt. Dafür ist (121) ein Beispiel: folgende für unseren Zusammenhang interessante Möglichkeiten der aktuellen Gliederung sind gegeben:

(12la) V sootvetstvii s programmoj sotrudniCestva meždu Sovetskim Sojuzom i Franciej na stancii "Saljut-6" / zaplanirovan rjad sovmestnych èksperimentov po kosmi Ceskomu materialovedeniju.

(121b) V sootvetstvii s programmoj sotrudnið̌estva meždu Sovetskim Sojuzom i Franciej / na stancii "Saljut-6" zaplanirovan rjad sovmestnych èksperimentov po kosmi ̌eskomu materialovedeniju.

Nach der aktuellen Gliederung in (12la) bestimmt die PP eine andere PP näher, genauer das in ihr enthaltene Nomen: sotrudniðestvo na stancii "Saljut-6". Eine weitere oberflächenrealisierung von (12la) ist damit:

(132) V sootvetstvii s programmoj sotrudni ̌estva meždu Sovetskim Sojuzom i Franciej, kotoroe imeet mesto na stancii "Saljut-6", zaplanirovan rjad sovmestnych èksperimentov.

In (121b) gehört die PP zum Bereich des Rhema, so verstanden, bestimmt sie das finite Prädikat näher: "na stancii "Saljut-6" zaplanirovan ..."

Die Mehrdeutigkeit von (121) kann zu Mißverständnissen führen: Die Lesart (12la) ist nicht unter die von (12lb) subsumierbar: das angesprochene zwischenstaatliche Abkommen wird mehr umfassen als die gemeinsamen Vorhaben auf der welt- 
raumstation - d.h. für die Aufhebung dieser Art semantischer Ambiguität, sind pragmatische Faktoren - in diesem Fall Sachwissen - heranzuziehen.

Die Ableitungsgeschichte von Sätzen der Struktur: $\mathrm{S}_{\mathrm{OF}} \longrightarrow \mathrm{NP}^{\mathrm{P}} \mathrm{PP}_{\text {loc }} \mathrm{VP}$ läßt sich schematisch mit Hilfe von Einbettungs-Tf darstellen:

1) Prop $_{2}$ wird in Th ${ }_{1}$ von Prop ${ }_{1}$ eingebettet (s. Schema 2a)

2) Prop ${ }_{2}$ wird in $\mathrm{Rh}_{1}$ von Prop $_{1}$ eingebettet mit sich anschließender Permutations-Tf ( $s$. Schema 2b).

Schema 2a

funktionale Ebene der Ableitung
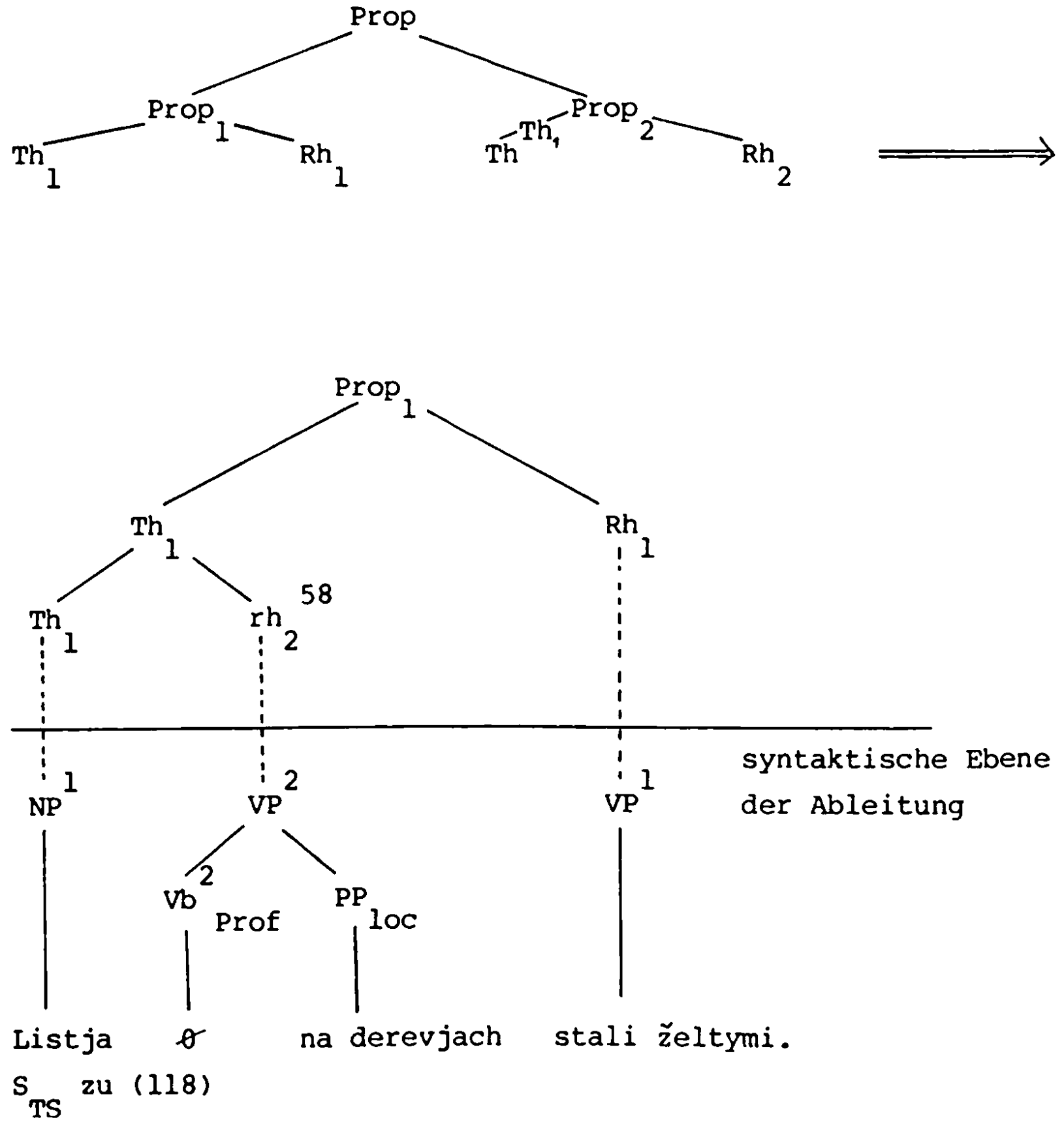

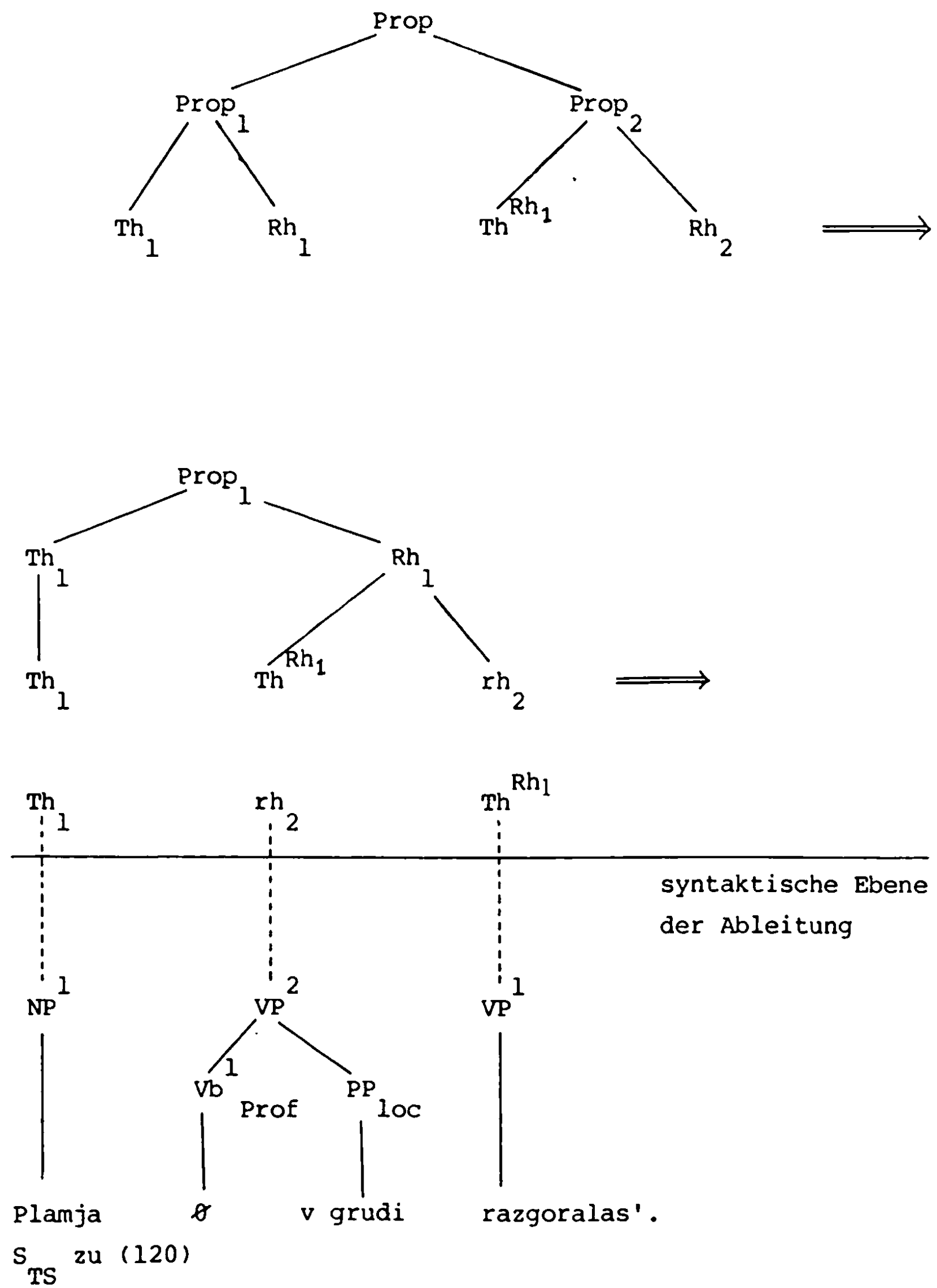


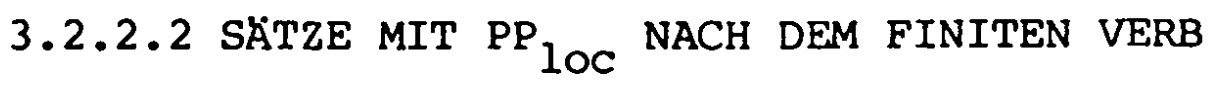

Im Unterschied $z u$ der Position der ${ }^{P P}{ }_{l o c}$ vor dem finiten verb, die aufgrund der variierbaren Grenze von Thema und Rhema vor oder nach der PP potentielle Mehrdeutigkeit der PP bedeutet, sind die Beziehungsmöglichkeiten der PP nach dem finiten Verb durch denselben funktionalen Faktor "Grenze" der aktuellen Gliederung in der Regel eindeutig das ließen bereits die. Analysen $z u$ oben (131) im besondern (131b) vermuten.

Die zur Diskussion stehenden Sätze haben die Struktur:

$\mathrm{S} \longrightarrow \mathrm{NP}^{1} \mathrm{VP} \quad \mathrm{NP}^{2}$

In der paradigmatisch starken Form der Aussage sind die funktionalen Gliederungen unserem Test entsprechend:

$\mathrm{Th}_{1} / \mathrm{Rh}_{1}: \mathrm{NP}^{1} / \mathrm{NP}^{2} \mathrm{NP}^{2} \quad \mathrm{Th}_{2} / \mathrm{Rh}_{2}: \mathrm{NP}^{1} \mathrm{VP} / \mathrm{NP}^{2}$

Im Fall der Positionen der PP ${ }_{10 \mathrm{C}}{ }_{2}$ dem finiten Verb tritt das Lokaladverbial $z$ wischen VP und $N P^{2}, d . h$. für die funktionale Gliederung auch an eine mögllche Grenze von Thema und Rhema. Die Beziehungsmöglichkeit(en), die sich damit für PP loc ergibt (ergeben) oder auch nicht, sollen ergänzend zu ( $13 \mathrm{l}$ ib) folgende Beispiele illustrieren: "/" markiert die möglich(en) aktuelle(n) Gliederung(en):

(133) Lavreckij slušal ee molča / i vertel v rukach / కljapu.

(134) On važno sžimal $v$ zubach / pustuju trubku.

(135) Žena boevogo oficera dolžna umet' / deržat' $v$ rukach / ruz'e.

(136) (Étim plamenem ja sogreju ves' mir. - ... Potom, potom - skazala ona.) A poka svari ... na svoem ogne / sosiski. 
Bei den Sätzen fällt auf, daß ungeachtet verschiedener möglicher T-C-A die PP ${ }_{l O C}$ das finite Verb näher bestimmt. Damit erhebt sich die Frage, in welchem Zusammenhang die objektsprachlichen Verhältnisse gesehen werden müssen, daß bei der Positon zwischen VP und NP ${ }^{2}$, die auch eine Grenzposition der T-C-A sein kann, die Prädikationsmöglichkeiten der PP ${ }_{\text {loc }}$ der genannten Restriktion unterliegen - stehen doch diese Fakten in deutlichem Gegensatz zu den Regeln über die Prädikationsmöglichkeiten der PP Grenze zwischen VP und NP ${ }^{1}$.

Die Frage berührt die Rolle der Adverbialen innerhalb der Regelmäßigkeiten der $\mathrm{T}-\mathrm{C}-\mathrm{A}$ überhaupt. Sie tiefer $\mathrm{zu}$ untersuchen, ist nicht Thema dieser Arbeit. Ich beschränke mich daher darauf, vorliegende Ergebnisse über die T-C-A auf das hier diskutierte Problem anzuwenden. Diese Analysen haben ergeben, daß die pauschal mit "Thema" und "Rhema" bezeichneten Glieder der Aussage präziser bestimmt werden können, wenn man versucht, die Strukturierung der Aussage als "kommunikative Dynamik", von der sich die kommunikative Funktion ableitet, $z u$ bestimmen. Die Ergebnisse stimmen einhellig darin überein, daß in russischen sätzen ${ }_{60}$ mit "normaler" Intonation die kommunikative Dynamik am Ende der Aussage maximal ist. Zur Beantwortung der oben gestellten Frage vergleichen wir an dem Beispiel oben (126) Endstellung der PP ${ }_{\text {loc }}$ und Stellung der PP ${ }_{\text {loc }}$ nach dem finiten Verb:

(131c) Ekspansivnyj M. Ataev nemedlenno otkazalsja ot svoej podpisi v priemno-sdatocnych dokumentach.

(13lb) ... otkazalsja v priemno-sdatoxnych dokumentach ot svoej podpisi.

Die Gegenüberstellung verdeutlicht, daß in Endstellung die

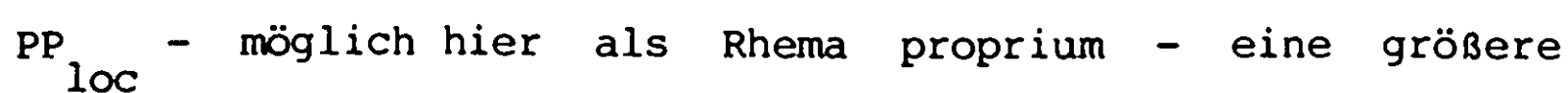
kommunikative Funktion im Satz besitzt als nach dem finiten Verb. In (131b) besitzt "ot svoej podpisi" im Verhältnis zu den übrigen Satzgliedern - damit auch $z u P_{\text {loc }}$ - die größere 
kommunikative Funktion (Entsprechendes gilt für o. (133 (136)). In diesem Zusammenhang muß m.E. die "Unverträglichkeit" einer näheren Bestimmung des Rhema proprium - hier der NP - durch ein Lokaladverbial, d.h. ein weiteres prädikat gemäß der tiefenstrukturellen Analyse gesehen werden. Damit sind für die stellung der PP ${ }_{\text {loc }}$ an "nichtletzter" Stelle "Zurücknahme", Abschwächung der kommunikativen Funktion und Disambiguierung ihrer funktionalen und syntaktischen Mehrdeutigkeit charakteristisch. 


\subsubsection{SÄTZE MIT PP ${ }_{\text {IOC IN ANFANGSSTELLUNG }}$}

Es wurde oben S. 103 erwähnt, daß für Sätze der Struktur $S \longrightarrow N P$ VP Endstellung der PP ${ }_{10 C}$ als verbreitet angesehen wird. Die Frage, in welchem Zusammenhang hier die Endstellung zu sehen ist, wurde zurückgestellt. Sie soll jetzt wieder aufgegriffen werden, da die Gegenüberstellung von Endstellung und Anfangsstellung der PP für die Klärung der mit diesen Positionen verbundenen Funktionen Aufschlüsse $z u$ geben scheint. Man vgl.:

( 19) Pervye zvezdy zazigalis' na rozovom nebe.

(139) Na rozovom nebe zažigalis' pervye zvezdy.

Gibt es einen Unterschied zwischen (19) und (139)? Wenn ja, worin liegt er begründet und wie kann man ihn beschreiben? Der erste Schritt bei der Anwendung des Prädikations-Tests, die Bildung von

$\mathrm{S} \longrightarrow \mathrm{NP}$ VP

ergibt, verstanden als expressiv neutraler Satz:

( 19') *Pervye zvezdy zažigalis'.

(139') Zažigalis' pervye zvezdy.

Wodurch ist das unterschiedliche Testergebnis bedingt? Sätze wie (139') sind in den Analysen zur Thema-Rhema-Gliederung (und auch in syntaktischen 64 Darstellungen) immer wieder zur Diskussion gestellt worden.

Wendet man auf (139') den Frage-Test zur Feststellung von Thema und Rhema an, so ergeben sich zwei Fragemöglichkeiten: 1. Cto zažigalos'? - Zažigalis' / pervye zvezdy.

2. Cto $\left\{\begin{array}{l}\text { slučilos' } \\ \text { bylo }\end{array}\right\}$ ? - Zažigalis' pervye zvezdy.

Die 2. Fragemöglichkeit und die mit ihr verbundene Bedeutung des Satzes interessiert hier. Dieser Satz gehört zu den themalosen Aussagen, u.z. zu der Subklasse, die durch ein semantisch "leeres" Prädikat, d.h. ein Prädikat, mit dem über 
das Argument NP keine im eigentlichen Sinn einer Prädikation charakterisierende Aussage gemacht wird, gekennzeichnet ist. Man vgl. "Gremit grom", "Blesnula molnija", "Svetit solnce", "Duet veter", etc. Mit diesen Verben können Aktionsartbedeutungen verbunden sein - s. oben "zažigalis' zvezdy" -, ihr Hauptmerkmal bleibt jedoch, daß sie das "Sein" oder das "Sosein" der NP benennen. Es handelt sich damit um primäre Aussagen, die im Russischen die syntaktische Form

$\mathrm{S}_{\mathrm{OF}} \longrightarrow$ VP (NP) haben, um Existentialsätze.

DiF Untersuchungen zu Existentialsätzen (Arutjunova, 1976, 221) haben ergeben, daß $z u$ der Aussage "Es gibt ein $X "$ ein "Ausschnitt Welt" oder "die Welt" gehört, in der " $x$ ist". Die Aussage über diesen "Ausschnitt welt" hat syntaktisch häufig die Form eines Lokaladverbials und steht dann am Satzanfang. Es ist vor allem dieser Fall der Verwendung von PP ${ }^{\prime}$, für den man die Beschreibung des Lokaladverbials als "Situationskulisse" (Adamec); "setting" (Sgall); "Ausgangspunkt" (Jaksche) für zutreffend bezeichnen kann. Diese Bestimmung der Funktion der PP trifft auch auf Sätze mit Prädikaten anderer semantischer Klassen zu (s.u. $119 \mathrm{f}$ ).

Uberträgt man die Erläuterungen zu (139') auf (19'), so wird plausibel, weshalb "Pervye zvezdy zažigalis'" als expressiv neutrale Aussage nicht üblich ist, ganz im Unterschied zu (19). Es bietet sich die Schlubfolgerung an, daß sich die Aussage in (19) komplementär $z u$ der in (139) verhält: die Hierarchie der Prädikate ist vertauscht, das "Sein" von $x$ wird vorausgesetzt und (19) macht eine Aussage darüber, "wo" x ist" (Tugendhat, 1976, 461):

(19) Pervye zvezdy zažigalis' / na rozovom nebe.

Ist die Bestimmung der Funktion von $\mathrm{PP}$ loc in Anfangs- und Endstellung als komplementär verteilt stichhaltig, so wäre damit auch ein methodisches problem gelöst: es fällt auf, daß der Thema-Rhema-Gliederungs-Test für eine funktionale Analyse der PP ${ }_{10}$ in Anfangsposition keine Ergebnisse liefert, ja 
eigentlich nicht anwendbar ist. Der Thema-Rhema-GliederungsTest setzt "etwas Allgemeines" oder "etwas Bestimmtes" voraus, mit dessen Nennung implizit gemeint ist, daB es "wo" ist. Nach unserer Analyse ist es dieses "wo", das die PP in Initialstellung explizit nennt. So verstanden handelt es sich bei (139) und (19) um primäre Aussagen (Tugendhat, 1976, $451 \mathrm{f})$, die sich gegenseitig voraussetzen, wobei für die sprachliche Formulierung der Unterschied beobachtet werden kann, daß (139') ein üblicher Satz ist - der "Ausschnitt Welt" muß nicht ausgedrückt sein. Umgekehrt wird klar, 1. weshalb (19') kein üblicher Satz ist, denn es fehlt das eigentliche Prädikat, da "zažigat'sja" zu "zvezda" kaum differenzierende Bedeutung hat; 2 . daß in den Sätzen der Struktur $S \longrightarrow$ NP VP die PP ${ }_{\text {loc }}$ in Endstellung verbreitet angetroffen wird; und 3 . daß bei einem wortstellungswechsel der PP ${ }_{l o c}$ von Endstellung zu Anfangsstellung wir einen Satz mit einer anderen Bedeutung erhalten. Damit trifft auch auf diese Position der PP die Regel $z u$, daß die Grenze von Thema und Rhema die tiefenstrukturellen Prädikationsmöglichkeiten der lokalen Prädikation limitiert, u.z. in der Weise, daß die PP ${ }_{\text {loc }}$ in Initialstellung als "Situationskulisse" für die ganze Aussage, als Rahmen "par excellence" fungiert; negativ ausgedrückt, daß mit ihr keine lokale Prädikation über ein oder mehrere bestimmte Argumente vollzogen wird.

Die dargestellten Verhältnisse sind besonders bei Sätzen mit Verben der genannten Subklasse - den Verben mit wenig spezifischen semantischen Merkmalen - evident; sie sind jedoch im Prinzip überhaupt auf Sätze der struktur $\mathrm{S}_{\mathrm{OF}} \longrightarrow$ NP VP PP loc anwendbar.

Zur Illustration zunächst noch einige ergänzende Beispiele zu der Verbklasse mit wenig spezifischen semantischen Merkmalen.

(140) V pustote i tił̌ne / zveneli క̌agi bogomol'cev. 
(141) Bogomol'cy prichodili ... Šagi ich zveneli / v pustote i tišine.

(143) V Karelii / sochranilas' drevnjaja naskal'naja Živopis'.

(143) Drevnajaja naskal'naja Żivopis' sochranilas' / $\checkmark$ Karelii.

(144) Na ee glazach / zablistali slezinki.

(145) I slezinki zablistali / na ee glazach.

(146) V každoj čerte ee smuglogo lica / igrala molodaja zizn'.

(147) Molodaja žizn' igrala / v každoj čerte ee smuglogo lica.

(148) V serdce / buševal ogon'.

(149) Ogon' buševal / v serdce.

(150) (Liza v neskol'ko dnej stala ne ta, kakoju on ee znal:) $v$ ee dviženijach, golose, $v$ samom smeche / zamełalas' tajnaja trevoga.

(151) Tajnaja trevoga zamečalas' / v ee dviženijach ...

(152) Ne uspela ona sojti vniz i pozdorovat'sja s materju, kak ǔe pod oknom / razdalsja konskij topot.

(153) Konskij topot razdalsja / pod oknom.

Die als komplementär einander zugeordneten Funktionen der PP loc in End- und Anfangsstellung mit den daraus sich ergebenden Restriktionen für ihre Verwendung liegen auch in Sätzen mit semantisch "vollwertigen" Prädikaten vor, wobei allerdings die tiefenstrukturellen und funktionalen verhältnisse aufgrund der zusätzlichen Prädikation durch das finite verb verdeckt sind. ${ }^{66}$ weitere Faktoren, die die Bildung komplementär sich entsprechender Sätze mit Lokaladverbialen in End- und Anfangsstellung sowie von Sätzen, die themalos oder in Thema und Rhema gegliedert sind, mitbestimmen, sind die syntaktischen Valenzen des Verbs und seine semantische Merkmalstruktur. Das muß bei der Analyse der folgenden Sätze berücksichtigt werden.

Beispiele für die Satzstruktur S $\longrightarrow$ NP VP: 
(1) Vor iscez / $v$ tolpe ljudej.

( 2) V tolpe ljudej / iscez / vor.

( 21) Liza postriglas' / V B... monastyre.

(154) V B... monastyre / postriglas' / monachinja let 20-i.

( 17) Mal'čik spal / $v$ dome storoža vodokaðki.

(155) V dome storoža vodokački / spal / mal'čik.

(156) Dvadcat' šest' nazvanij knig vyšli $v$ svet / v serijach izdatel'stva "Avrora".

(157) V serijach izdatel'stva "Avrora" / vyšli v svet / dvadcat' šest' nazvanij knig.

Von den vielen Beispielen aus Kontexten, in denen die PP in Anfangsstellung die Funktion der "Situationskulisse" erfüllt, hier noch folgender Beleg:

(158) (my) ...v toj samoj komnate, kotoruju chozjajka nazyvaet svoim salonom. ... v ètom salone / porjadoǩno vykrašeny poly i nedurny vypisnye oboi. ... drugoj stol, na nem / kipit serebrjanyj samovar; ... okolo zatoplennogo kamina / rasstavleny kresla.

Prädikate wie die in den angeführten Sätzen bilden auch geteilte Aussagen mit finitem Verb als Rhema; die Funktion der PP ${ }_{\text {loc }}$ bleibt wie oben beschrieben:

(159) V tolpe ljudej vor / isčez.

(160) V B... monastyre Liza / postriglas'.

(161) V dome storoža vodokaðki mal'čik / spal.

Bei einer Reihe von Verben, die Sätze mit der Struktur $S \longrightarrow$ NP VP bilden, ist der Aussagetyp wie in (159) - (161) mit der Aussagegliederung: $\mathrm{PP}{ }_{\text {loc }} \mathrm{NP} / \mathrm{VP}$ wenn nicht blockiert, so doch nicht üblich, was ${ }^{10 c}$ möglicherweise auf bestimmte Regelmäßigkeiten syntaktischer strukturen mit intransitiven Verben hinweist ( $Z$ immermann, 1978, besonders $76 \mathrm{f}$ ) - so 
ließe sich erklären, daß hier Sätze mit intransitiven Verben und verben im Passiv Gemeinsamkeiten aufweisen (vgl. 162-164 und 165-173).

Sätze mit diesen Verben sind am Textanfang oder Beginn eines Absatzes verbreitet; dabei ist für sie typisch, daß sie mit einer PP ${ }_{\text {loc' seltener mit einer PP }}$ temp eingeleitet werden:

(162) V ... sel'skochozjajstvennoj akademii vystupil predsedatel' Gosudarstvennogo Soveta PNR Jablonskij.

(163) Predsedatel' Gosudarstvennogo Soveta PNR vystupil v ... sel'skochozjajstvennoj akademii.

$(164) * V$... sel'skochozjajstvennoj akademii predsedatel'

Gosudarstvennogo Soveta PNR Jablonskij vystupil.

(165) V Dvorce kul'tury "Družba" bylo otmeceno 120-letie so dnja roždenija Cechova.

(166) 120-letie so dnja roždenija Cechova bylo otmełeno $\mathrm{v}$ Dvorce kul'tury "Družba".

(167)*V Dvorce kul'tury "Družba" 120-letie so dnja roždenija Cechova bylo otmečeno.

(168) V objazatel'stvach zapisany tysjači tonn sverchplanovogo alljuminija.

(169) Tysjači tonn sverchplanovogo alljuminija zapisany $v$ objazatel'stvach.

$(170)(*) V$ objazatel'stvach tysjači tonn sverchplanovogo alljuminija zapisany.

(171) Na vystavke predstavleny raboty sovremennych chudožnikov.

(172) Raboty sovremennych chudožnikov predstavleny na vystavke.

$(173)(*)$ Na vystavke raboty sovremennych chudožnikov predstavleny.

Sätze mit der Satzstruktur $\mathrm{S} \longrightarrow \quad \mathrm{NP}^{1}$ VP $\mathrm{NP}^{2}$ sind in themalosen Aussagen nur mit unpersönlichem Prädikat, d.h. mit 
getilgter $\mathrm{NP}^{1}$ vertreten (Krylova, Chavronina, 1976, 26; 207 f).

Die Darstellung über die Anfangs- und Endstellung der PP 10 ' $^{\prime}$ abgeleitet aus Sätzen der struktur $S \longrightarrow \mathrm{NP}^{1} \mathrm{VP}$, ist auf diese Satzstruktur ebenfalls anwendbar. Man vgl.:

(174) V portu razgružajut parochody (Beispiel aus Krylova, Chavronina, 1976, 32).

(175) Parochody razgruzajut $v$ portu.

(176) V mikrorajonach strojat novye doma.

(177) Novye doma strojat v mikrorajonach.

In geteilten Aussagen hat die $\mathrm{PP}$ loc in Initialstellung ebenfall die Funktion einer Situationskulisse und ist von der Finalstellung entsprechend $\mathrm{zu}$ unterscheiden. Man vgl.:

(178) Za stolom general potževal ego chorošim lafitom.

(179) V Afganistane politið̌eskie preobrazovanija otkryvajut put' dlja rassirenija svjazej s SSSR.

(180) Na kosmičeskoj orbite kosmonavty nacinajut svoju vachtu v 6 Casov.

(181) V étom zdanii A.P. Čechov provel 11 gimnaziðeskich let.

Die Untersuchungen $z \mathrm{u}$ der $\mathrm{PP}{ }_{10 \mathrm{C}}$ in Anfangsstellung bestätigen die in Kap. 3.2.1 abgeleitete Regel, daß die Grenze von Thema und Rhema restriktiv für den Prädikationsbereich des Lokaladverbials wirkt: wechselt die PP ${ }_{10 C}$ von der Endstellung in die Anfangsstellung, so erhalten wir einen Satz mit anderer Bedeutung. Diese Verhältnisse sind besonders evident bei Existentialsätzen. Darüberhinaus führt die Analyse von Existentialsätzen $z u$ weiterführenden Ergebnissen, die - wenn auch in verdeckter Form - bei einfachen Strukturen überhaupt zu erkennen sind: bei Anfangs- und Endstellung der PP handelt es sich um eine komplementäre verwendung der Lokalprädikation im Satz, die oberflächenstrukturell so geregelt ist, daß in Existentialsätzen, in themalosen Sätzen oder 
in Sätzen mit VP als Rhema die vorausgesetzte Prädikation "Loc" am Satzanfang steht; ist umgekehrt PP loc Rhema des Satzes, steht diese - gemäß den bekannten wortstellungsregeln der aktuellen Satzperspektive im Russischen - in Endstellung. Die so gewonnenen Ergebnisse liefern Argumente für die Lösung zweier Fragekomplexe:

1. Für Oberflächensätze mit PP loc in Anfangsstellung liefert der Prädikations-Test keine plausiblen Ergebnisse; bzw. nur solche Ergebnisse, die keinen erkennbaren Unterschied zu Sätzen mit PP ${ }_{\text {loc }}$ in Endstellung ergeben. Man vgl.:

\section{(2) V tolpe ljudej isčez vor. \\ Iscez vor. Éto imelo mesto $v$ tolpe ljudej. (Éto) imelo mesto $v$ tolpe ljudej, gde is}

Es scheint, daß für diese Sätze die generativtransformationelle Methode keine dem intuitiven Sprachverständnis entsprechende adäquate Beschreibung leistet. Der Nachweis der komplementären Beziehung zwischen End- und Anfangsstellung der $\mathrm{PP}$ loc deckt den Erklärungszusammenhang auf, weshalb die generativ-transformationelle Methode hier nicht "greift" - in dem Sinn, daß es nicht gelingt, die Ableitung der tiefenstrukturellen Verhältnisse zu dem oberflächensatz nachzumodellieren, was am deutlichsten die Analyse von Existentialsätzen zeigte:

Existentialaussage und Lokalprädikation sind Prädikationen, die sich gegenseitig voraussetzen, im Unterschied zu Prädikationen, die einander nebengeordnet, übergeordnet etc. sind. $P P$ in Anfangsstellung macht keine Aussage über " $X$ " in dem sinn "' $X$ ' ist dort", sondern nennt explizit den "Ort", der in der Aussage "' $X$ ' ist" implizit immer enthalten ist; d.h. auch im Prädikations-Test vorausgesetzt wird. Die tiefenstrukturelle Analyse von Lokaladverbialen mit Hilfe des Prädikations-Tests stößt bei den Fällen PP ${ }_{\text {loc }}$ in Anfangsstellung an eine methodologische Grenze. Analysen der Sätze wie (19) und (139) - s.o. S. 117 ff - mit Hilfe des Thema- 
Rhema-Gliederungs-Tests führten $z u$ entsprechenden Ergebnissen.

In diesem zusammenhang ist auch der 2. Fragenkomplex zu sehen. Er betrifft die Bestimmung der Funktion der PP in Anfangsstellung im Rahmen der aktuellen Satzperspektive, die Diskussion zu der Frage:

ist die PP "setting", auch "Situationskulisse" genannt, oder Thema? Wenigstens zu den Existentialsätzen konnte deutlich gemacht werden, daß hier die PP den "Ausschnitt Welt" nennt, in dem "X (so) ist", und daß damit die PP nicht Thema ist, sondern explizit nennt, was in der Existentialaussage implizit enthalten ist. Damit legen die tiefenstrukturellen Analysen der Prädikation für diesen Fall die Bestimmung der Funktion der PP ${ }_{\text {loc }}$ in Initialstellung als "setting" nahe.

Abschließend seien die Ergebnisse dieses Kapitels über die freien Lokaladverbiale zusammengefaßt.

Die Untersuchungen erfolgten mit zwei methodisch verschiedenen Beschreibungsverfahren. Der auf dem Hintergrund der generativ-transformationellen Analyse abgeleitete Prädikations-Test fragt nach syntaktischen Relationen, die bei Erweiterung von Sätzen mit $\mathrm{PP}$ loc die Bedeutung dieser Sätze mitbestimmen, und untersucht diese. Als Ergebnis konnte die für Lokalprädikationen charakteristische Mehrdeutigkeit ihrer syntaktischen Relation zu den übrigen Satzgliedern (Schema 1, S. 70) festgestellt werden. Diese syntaktische Mehrdeutigkeit ist durch die Eigenart der lokalen Prädikation - ihrer Möglichkeit, über verschiedene kategoriale Bereiche, über Individuenvariable wie über Ereignisvariable zu prädizieren bedingt. Dieses Charakteristikum der Prädikation gilt von den in dieser Arbeit untersuchten Adverbialen nur für die lokalen und unterscheidet damit diese objektsprachlich in ihrer Funktion und metasprachlich für die Beschreibungsmethode von den temporalen und modalen (s. Kap. 4, $127 \mathrm{ff}$ und Kap. 5, 183 ff). 
Für eine plausible Beschreibung der Funktion des Lokaladverbials im Satz reicht die generativ-transformationelle Beschreibung nicht aus - was m.E. in dieser weise nicht für die temporalen und modalen Adverbialen zutrifft -, da sie keine Kriterien für die bei der Verwendung von Lokaladverbialen offensichtlich sich regelmäBig vollziehende Disambiguierung der syntaktischen Mehrdeutigkeit liefert.

Auf der Suche nach solchen Kriterien konnten regelmäßige Beziehungen zwischen der Verwendung der PP und der aktuellen Satzperspektive aufgedeckt werden. Mit Hilfe des Thema-Rhema-Gliederungs-Tests wurde die Grenze von Thema und Rhema als Kriterium für die Disambiguierung der syntaktischen Mehrdeutigkeiten der PP ${ }_{\text {loc }}$ ermittelt (Schema 2, S. 102 und $2 a / 2 b, S$. 112 f) Daraus ließen sich Wortstellungsregeln für die PP im Satz ableiten, nach denen Endstellung (s. Kap. $3.2 .1)$, Stellung vor dem finiten verb (3.2.2) und nach diesem (3.2.3) und Anfangsstellung (3.2.4) für die Funktion der ${ }_{\text {Ioc }} \mathrm{zu}$ unterscheiden sind. 


\section{TEMPORALE ADVERBIALE}

In der neueren ${ }^{1}$ Linguistik wurde in generativen Grammatiken und in von diesen inspirierten Arbeiten den temporalen Adverbialen eine Reihe einschlägiger Darstellungen gewidmet. Schon aus den Titeln geht hervor, daß die linguistische Darstellung der temporalen Adverbialen selbst in Arbeiten mit vergleichbarem Beschreibungsansatz unter unterschiedlichen Gesichtspunkten geführt wurde. Ein vergleichender Uberblick über Fragestellungen und zielsetzungen neuer Arbeiten $z u$ lokalen Adverbialen einerseits und temporalen andererseits ergibt, dab bei den lokalen das Interesse vorrangig dem Unterschied und möglichen Unterscheidungskriterien von "obligatorischen" und "fakultativen" Lokaladverbialen gilt (s.o. Kap. 2, bes. 2.1.2, s. $30 \mathrm{ff}$ ). Bei den temporalen Adverbialen ist dieses Problem "obligatorische/fakultative" Ergänzung nicht eigentlich relevant. Das hängt wohl damit zusammen, daß empirische Arbeiten zur Verbvalenz auf keine den Verben "liegen", "gehen" entsprechenden repräsentativen Verbklassen gestoßen sind, für die ein temporales Adverbial syntaktisch und/oder semantisch obligatorisch wäre.

Jedoch ist eine andere - wie ich meine - zentrale Problemstellung den sonst recht unterschiedlichen Arbeiten, die unter Anm. 2 aufgeführt sind, gemeinsam. Sie analysieren die temporalen Adverbiale als fakultative Satzergänzungen, die das Prädikat modifizieren.

Das Problem, wie die syntaktischen Strukturen der Sätze mit temporalen Adverbialen $z \mathbf{u}$ beschreiben sind, behandeln die Arbeiten insofern gleichartig, als sie die temporalen Adverbiale als Oberflächenstrukturen von einer zugrundegelegten Tiefenstruktur ableiten, die ihrerseits die Form eines Satzes hat. 
Bei den Fragen jedoch nach den Gliedern dieses Satzes, ihrer Beziehungen zueinander, im besonderen der der Temporaladverbiale $z u$ den übrigen Satzgliedern, woraus sich enventuelle Regelmäbigkeiten für die strukturen des oberflächensatzes ergeben, gehen Argumentation und Lösungsvorschläge auseinander. Diese Lösungsvorschläge sollen im Folgenden insoweit diskutiert und ausgewertet werden, als sie einen Beitrag zuder Frage liefern, ob - am Material des Russischen - Regelmäßigkeiten für die Erweiterung von Sätzen mit temporalen Adverbialen $z u$ beobachten sind. Diese Problemstellung $z$ ielt auf die oben angesprochene Frage, auf welche syntaktischen Verhältnisse der zur Diskussion stehenden Sätze diese Regelmäßigkeiten schließen lassen. Es wird so eine einheitliche Fragestellung bei den drei hier untersuchten fakultativen Adverbialen verfolgt.

Der erwähnte Dissens in der Literatur über den den temporalen Adverbialen zugrundezulegenden Satz hängt m.E. damit zusammen, daß empirische Untersuchungen zwei Hauptklassen temporaler Adverbiale ermittelt haben:

a) temporale Adverbiale, die ein "Ereignis" zu (einer)

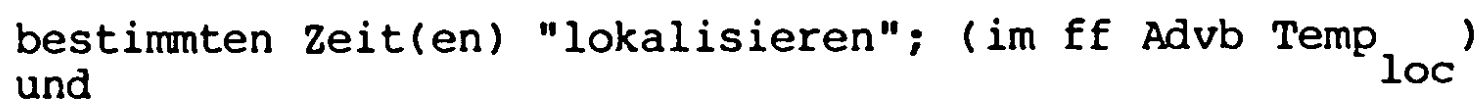

b) temporale Adverbiale, die die "Dauer" eines "Vorganges" oder "Zustandes" quantííizieren" (im ff Advb Temp quant).

Zu den Ergebnissen dieser Untersuchungen gehört, daß bestimmte RegelmäBigkeiten für die Erweiterung von Sätzen mit Temporaladverbialen festgestellt werden. Man vgl. für das Russische:

(1) Vstreca predstavitelej profsojuzov imeet mesto $v$ marte.

(2) *Vstreca predstavitelej profsojuzov imeet mesto dve nedeli.

(3) Predstaviteli profsojuzov vstrečajutsja v marte.

(4) Predstaviteli profsojuzov vstrečajutsja dve nedeli ... 
(5)*Sidenie ego na meste dekana imelo mesto $\mathrm{v}_{7}$ prošlom godu.

(6) On sidel na meste dekana $v$ prošlom godu.

Bei den Beispielen handelt es sich um Sätze, von denen die einen mit AdvbTemp ${ }_{10 c}(1,3,5,6)$, die übrigen mit $A d v b$ Temp quant erweitert sind.

Die Sätze machen z.B. auf die Frage aufmerksam, wie die Grammatikalität von (4) mit Advb Temp und (6) mit Advb Temp loc und die Nichtgrammatikalität der nominalisierten Form dieser Sätze ( 2 und 5) zu beschreiben sind.

Die Arbeit von wunderlich (1970) geht solchen Fragen nicht im Detail nach. Dennoch ist diese Arbeit für unsere Fragestellung von Interesse, da sie Argumente für die TS von temporalen Adverbialen, lokalisierenden und quantifizierenden, diskutiert und Vorschläge für eine TS ausarbeitet. Die Untersuchungen führen $z u$ dem Ergebnis, daß Wunderlich die beiden Klassen temporaler Adverbiale aus einer TS ableitet. Man vgl. die Darstellung zu folgenden Sätzen (op. cit. 285):

"(328a) Die Türken belagerten Wien so lange, wie ihr Proviant reichte.

(328b) Die Belagerung Wiens durch die Türken dauerte so lange, wie die wiener sich verteidigen konnten.

(328c) Die Belagerung Wiens durch die Türken fand zur gleichen zeit statt wie die Besetzung Luxemburgs."

Bei Wunderlich werden diese Sätze aus einer TS mit folgenden Phrasenstrukturmarkern abgeleitet: 
F 51

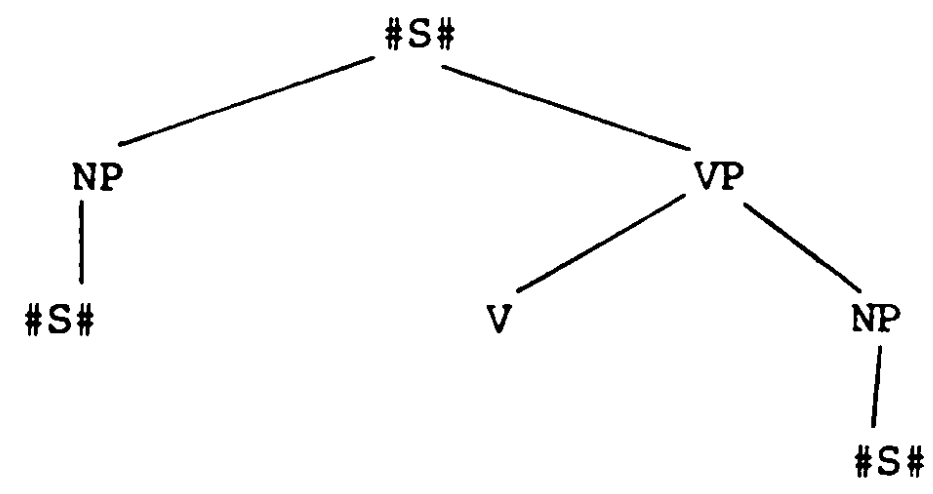

Für die Ableitung von Sätzen mit Temporaladverbialen $" 1+$ Dauer)" und "(- Dauer)" wird darauf hingewiesen, "daß beide vorkommenden NP zu S expandiert" werden. ... "Damit ist:

1. ihre strukturelle verwandschaft erklärt, und

2. die Parallelität $z u$ den Maßangaben gewährleistet" (ibid. 285).

Jedoch muß wunderlich einschränkend zugeben: "daß $\ldots$ der Weg, wie aus der Struktur in (F 51) der Ubergang zu den von mir im Detail beschriebenen syntaktischen Zusammenhänge zu finden ist, unklar ist. Mit der struktur in (F 51) sollte vor allem eine Generalisierung erreicht werden, nämlich eine einheitliche Beschreibung aller zeitadverbiale im Paradigma der Maßangaben" (ibid. 286).

Es scheint symptomatisch für syntaktisch orientierte Grammatiken, daß sie die Knoten, die über die Beziehung von Sätzen (hier die beiden \#S\#) auf der Ebene der Tiefenstruktur Aufschluß geben könnten, nicht näher bestimmen. So wird für (F 51) m.E. die Frage nach der Beziehung der beiden \#S\# zueinander dadurch erst gar nicht relevant oder problematisch, da diese \#S\# von nicht weiter untersuchten NP dominiert werden, die auf unterschiedlichen Stufen der Ableitungshierarchie stehen und deren Beziehungen zueinander ebenfalls nicht diskutiert werden ( $s$. hierzu auch die selbstkritische Argumentation von Wunderlich (loc. cit. 286), auf die ich nur verweise). 
Weiterführende ${ }^{10}$ Lösungsvorschläge für die Beschreibung der Beziehungen von Sätzen der TS liefern prädikatenlogisch orientierte Arbeiten mit ihrer zentralen Fragestellung nach der Relation zwischen Prädikat und seinen möglichen Argumenten (vgl. auch oben die Darstellung der lokalen Adverbiale, 2.B. S. $66 \mathrm{ff}$ ). Wendet man diese Fragestellung auf (F 51) an, wird deutlich, daß Untersuchungen der beiden die \# S \# dominierenden NP unter prädikatenlogischer Fragestellung erforderlich sind.

Die Analysen von Bartsch (1972) zu temporalen Adverbialen führen $z u$ dem Ergebnis, daß den beiden Klassen temporaler Adverbiale - Advb Temp und Advb Temp - verschiedene Klassen logischer Relationen von Präquakat und Argument zugeordnet werden müssen: Die Klasse Advb Temp ${ }_{\text {loc }}$ ist dadurch charakterisiert, daß das Prädikat "ereignet sich 'wann'" ausschließlich Argumenten der Klasse "Ereignis/Umstand" zugesprochen wird (vgl, oben die Beispiele (1), (3) und (6)). "Es ist möglich, die Relationen 'während', 'zügleich', 'nach' und 'vor', die zwischen Ereignissen und Umständen bestehen, durch die Angabe von zeitintervallen und Relationen zwischen diesen $z u$ ersetzen. Dabei müßten aber diese Relationen zwischen Zeitintervallen, wie ' $t_{i}=t_{j}$ ' oder ' $t_{i}<t_{j}$ ' letztlich doch auf die Gleichzeitigkeit ${ }_{j}^{i}$ der Aufeinanderfolge von Ereignissen, die der Bestimmung von $t_{i}$ und $t$ dienen, zurückgeführt werden. Zeitintervalle und zeitpunkte (als Grenzen von Intervallen) sind nur durch Klassen von Ereignissen angebbar, die durch konventionell festgesetzte Ereignisse oder Ereignisfolgen repräsentiert werden. Solche sind Sonnenumläufe der Erde ..." (loc. cit. 118).

Die Klasse Advb Temp quant macht im Unterschied zu AdvbTemp Angaben über die Länge des zeitintervalls von Ereignissen (vgl. (4)). Sie gehört damit zu der Klasse der Maßangaben wie Größe, Gewicht etc. und bedarf einer eigenen Untersuchung. 
Die beiden Klassen von Temporaladverbialen können auch gemischt verwendung finden - es handelt sich dabei um Mischkonstruktionen, " in denen temporale Relationen mit Quantifikationen verbunden sind. Sie können im zusammenhang mit der Untersuchung der quantifizierenden und graduierenden Adverbiale formalisiert werden" (Bartsch, 120).

Mit dieser zuordnung der temporalen Adverbiale zu zwei unterschiedlichen Klassen von Relationen zwischen Prädikat und Argument gibt Bartsch einen Beschreibungszusammenhang für die $z u$ den Sätzen $(4,6$ und 2, 5) aufgeworfene Frage: "dve nedeli" ist ein Ausdruck für Advb Temp ; damit verstößt (2) gegen die Regeln, die aus der prădikation Temp resultieren, indem ein Prädikat der objektsprache verwendet wird - nämlich "stattfinden" - das in der Regel als Proform bei der Verwendung der anderen Klasse temporaler Adverbiale

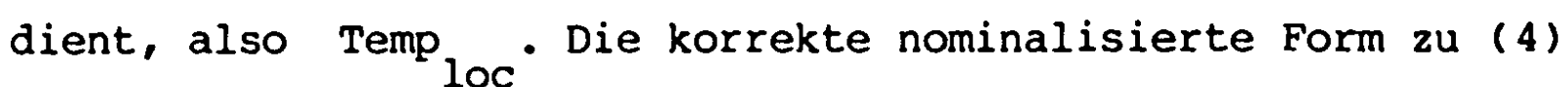
mit Advb Temp "dve nedeli" ist: quant

(7) Vstreča predstavitelej profsojuzov dlilas' dve nedeli.

In (5) ist die Relation zwischen den Gliedern der VP, nämlich

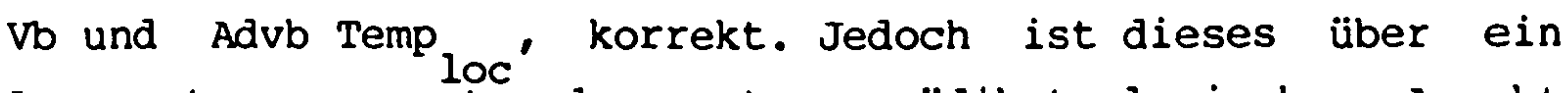
Argument ausgesagt, das unter prädikatenlogischem Aspekt nicht zu der Klasse "Ereignis/Umstand" gehört, sondern zu der Klasse "Zustand"; (5) ist damit nicht die nominalisierte Form von (6). Diese ist vielmehr unter Beibehaltung der satzsemantischen Relationen:

(6a) To obstojatel'stvo, cto on sidel na meste dekana, imelo mesto $v$ proslom godu.

(5) kann auch so korrigiert werden, daß Advb Temp durch Advb Temp quant ausgedrückt wird: man vgl.:

(5a) Sidenie ego na meste dekana prodolłalos' $v$ tełenie pjati let. 
Die Schlußfolgerungen, die sich aus der Berücksichtigung prädikatenlogischer Analysen für die Beschreibung der TS russischer temporaler Adverbiale ergeben, kommen unten ( $S$. $135 \mathrm{ff})$ zur Sprache.

Vorher sollen die für unsere Fragestellung relevanten Ergebnisse der Untersuchungen von Krause (1977) referiert werden, die zwar aus einer Analyse deutscher Geschehensverben gewonnen wurden, die aber - mit gewissen Einschränkungen, die sich auf die lexikalischen Besetzungen der Geschehensverben beziehen - für das Russische m.E. übernommen werden können.

Krause überprüft an einem repräsentativen Korpus vor allem die Klassifikation von Bartsch (1972). Er geht im besonderen der Frage nach: Stimmt es, daß die Prädikationsmöglichkeiten der Advb Temp ${ }_{\text {loc }}$ in der Weise limitiert sind, daß sie nur über Argumente der Klasse "Ereignis/Umstand" ausgesagt werden können. Seine Ergebnisse machen deutlich, daß sowohl die restriktive Position von Bartsch als auch die generalisierende Konzeption von wunderlich teilweise revidiert werden müssen. Die Geschehensverben lassen sich klassifizieren, wobei von maßgeblicher Bedeutung ihre möglichen Argumente sind. Es läßt sich feststellen, daß die bisher vorliegenden wortsemantischen Untersuchungen und Klassifikationsversuche der Uberprüfung durch ein umfangreiches Korpus nicht standhalten (Krause, 1975).

Unter dem Gesichtspunkt ihrer möglichen Argumente lassen sich die Geschehensverben in folgende Untergruppen einteilen:

"a) geschehen; b) vor sich gehen; c) stattfinden ...

Die Untergliederung richtet sich bei a) und c) ... deutlich nach dem Argument: In Sätzen mit 'geschehen' wird das Adverb über Ereignisse oder Vorgänge prädiziert, bei 'stattfinden' /'sich ereignen' nur über Ereignisse"(loc. cit. 224). 
Für die Ableitung der TS der Temporaladverbialen ist bei Berücksichtigung der Ergebnisse der Arbeit von Rrause die Position wunderlichs von einer gemeinsamen TS für Advb Temp loc und Advb Temp quant nicht haltbar. Aber auch die Analyse von Bartsch mü insoweit eingeschränkt werden, als in natürlichen Sprachen lokalisierende temporale Adverbiale über Argumente der Klassen "Ereignis" und "Vorgang" ausgesagt werden können. Das hat in einer linguistischen Untersuchung Konsequenzen für die metasprachliche Bestimmung des Prädikats, von der auch abhängt, in welchem Umfang objektsprachliches Material plausibel erfaßt werden kann.

Aufgrund der Zugehörigkeit von Advb Temp loc und Advb Temp $z u$ verschiedenen Klassen modifizierender Ausdrücke, für quant jeweils eigene Untersuchungen notwendig sind, wie auch wegen der besonderen Lage des Studiums russischer temporaler Adverbiale, für die generative Untersuchungen der satzsemantischen Verhältnisse m.w. fehlen, beschränke ich diese Arbeit auf die Untersuchung der Klasse Advb Temp ${ }_{10 c}{ }^{\circ}$ 
4.1 DAS TEMPORALADVERBIAL IN DER FUNKTION EINER PRÄDIKATION

4.1.1 ABLEITUNG DER TIEFENSTRUKTUR (TS) VON $\mathrm{S}_{\mathrm{OF}} \longrightarrow$ $\mathrm{NP}^{1} \mathrm{VP}^{1}(\mathrm{X}) \mathrm{PP}_{\text {temp }}(1)$

Bei der Untersuchung dieser Klasse PP ergeben sich zwei Subklassen, die sich oberflächenstrukturell dadurch unterscheiden, daß in der einen Klasse das Nomen der PP ein Ausdruck des zeitsystems ist (im folgenden NP zeit), in der anderen Klasse ein Nomen, das in seiner wortbildung in gewisser (unterschiedlicher) Relation $z u$ dem entsprechenden verbalen Ausdruck steht (im folgenden NP verb).

Für die Analyse der Lokaladverbiale mußten neben dem Prädikations-Test der Negations- und der Dialog-Test angewendet werden, mit deren Hilfe die unterschiedlichen Klassen: fakultative und obligatorische Lokaladverbiale bestimmt wurden. Wie bereits erwähnt (vgl. S. 127) ist dieses Problem für die Temporaladverbiale nicht relevant. Für die Feststellung der expliziten Beziehungen der PP $z u$ den übrigen Satzgliedern können wir uns vorläufemp auf den Prädikations-Test beschränken und wenden ihn zunächst auf die Klasse der $\mathrm{PP}_{\text {temp }}$ mit $\mathrm{NP}$ zeit an.

(8) Chimik Mendeleev podnjalsja $v$ tot pamjatnyj den' $v$ vozduch na vozdušnom sare.

\section{Prädikations-Test}

$S_{\text {TS }}^{l}$ : Chimik M. podnjalsja v vozduch na vozdusnom కare.

$\mathrm{S}_{\text {TS }}^{\text {TS }}$ Byl tot pamjatnyj den'.

$\mathrm{S}^{3} \mathrm{TS}$ : Podjatie ego $\mathrm{v}$ vozduch imelo mesto $\mathrm{v}$ tot pamjatnyj den'. 
Dieses Ergebnis findet allgemeine Bestätigung bei der Uberprüfung von Sätzen mit Advb Temp ${ }_{1 o c}$; die Ebene von $\mathrm{S}^{3}$ TS ist die für unsere Fragestellung endscheidende. Bei den Beispielen dieser Klasse von ${ }^{P P}$ temp mit ${ }^{2}{ }_{\text {zeit }}$ können wir uns auf die Untersuchung von $s^{l}$ tS und $s^{3}$ TS beschränken. ${ }^{15}$ Man vgl. weitere Beisp.:

(9) My vstretilis' s drugom detstva $v$ vychodnoj den'. $\mathrm{s}^{\mathrm{l}} \mathrm{TS}:$ My vstretilis' $\mathrm{s}$ drugom detstva.

$s_{\text {TS }}^{3}$ : Naša vstreča imela mesto $v$ vychodnoj den'.

(10) Ėtot fil'm vychodil na ékrany $v$ prošlom mesjace. $S_{\text {TS }}^{1}$ : Étot fil'm vychodil na ékrany.

$\mathrm{S}^{3} \mathrm{TS}$ : Vychod étogo fil'ma na èkrany imel mesto $\mathrm{v}$ prošlom mesjace.

(11) Ja vernulsja iz poezdki $v$ poslednij den' kanikul. $\mathrm{S}_{\mathrm{TS}}^{l}$ : Ja vernulsja iz poezdki.

$\mathrm{s}^{3} \mathrm{TS}$ : Moe vozvraščenie iz poezdki imelo mesto $\mathrm{v}$ poslednij den' kanikul.

(12) Ėta statuetka sozdavalas' neizvestnym masterom $v$ v bronzovom veke.

$\mathrm{S}_{\text {TS }}^{1}$ : Éta statuetka sozdavalas' neizvestnym masterom.

$\mathrm{S}_{\mathrm{TS}}^{3}$ : Sozdanie ètoj statuetki neizvestnym masterom imelo mesto $\mathrm{v}$ bronzovom veke.

(13) Amerika otkryvalas' Christoforom Kolumbom v vek velikich geografiðeskich sobytij.

$S_{T S}^{l}$ : Amerika otkryvalas' Christoforom Kolumbom.

$\mathrm{s}_{\mathrm{TS}}^{3}$ : Otkrytie Ameriki Christoforom Kolumbom imelo mesto $v$ vek velikich geografißeskich sobytij.

(14) Na vtoroj den' on priznalsja, tto perepisyvaetsja s Klavdiej Michajlovnoj. $\mathrm{S}_{\mathrm{TC}}^{1}$ : On priznalsja, čto ... 

$\begin{aligned} \mathrm{S}^{3} \mathrm{TS} & \text { Ego priznanie o tom, tto .... imelo mesto na vtoroj } \\ & \text { den'. }\end{aligned}$

(15) V detstve i otrołestve Blok pisal korotkie rasskazy i stichi.

$S_{\text {TS }}^{l}$ : Blok pisal korotkie rasskazy i stichi.

$\mathrm{S}_{\mathrm{TS}}$ : Pisanie korotkich rasskazov ... imelo mesto $v$ detstve i otrołestve.

(16) Ved' imenno èti rebjata rasstreljali na dnjach 300 amerikanskich plennych.

$S_{\text {TS }}^{l}$ : Éti rebjata rasstreljali 300 amerikanskich plennych. $S_{\text {TS }}^{3}$ : Rasstrel amerikanskich plennych imel mesto na dnjach.

Die Ergebnisse des Prädikations-Tests führen $z u$ folgenden Feststellungen:

Die den Sätzen (8-16) zugrundeliegende Struktur ist:

$\mathrm{S}_{\mathrm{TS}}^{3}: \mathrm{NP}^{\mathrm{VP}}$ imet' mesto Advb Temp ${ }_{\text {loc }}$

Diese ist abgeleitet aus der Verknüpfung von:

$\mathrm{S}_{\mathrm{TS}}^{1}: \mathrm{NP}^{\mathrm{l}} \mathrm{VP}^{1}$

$\mathrm{S}_{\mathrm{TS}}^{2}$ : $\mathrm{NP}_{\text {Prof }}$ byt' $\mathrm{NP}_{\text {zeit }}$

was an der Subjekt-NP von $s^{3}$ (NS $\left.{ }^{V^{1}}\right)^{1}$ ablesbar ist. Mit Hilfe des Tests können nun Aussagen über die Relation der $\mathrm{PP}_{\text {temp }} \mathrm{zu}$ den übrigen Satzgliedern gemacht werden:

1. Von den ermittelten Sätzen der $T S$ ist dafür $S^{3}$ aufschlußreich. Denn aus den möglichen Relationen, die $S^{l}$ TS $\mathrm{S}^{2}$ TS eingehen können, wird die temporale Relation dadurch gewählt, daß $\mathrm{S}^{2}$ TS nach Nominalisierungs-TF (vgl. unten $\mathrm{S}$. 141, im Schema $3 a$ der Knoten NP Adv' und infolge seiner lexikalischen Besetzung mit NP zeit eine temporale Bestimmung $z$

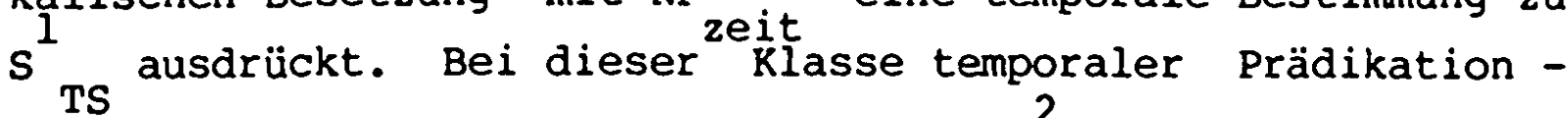
mit NP ${ }_{\text {zeit }}$ - ist die subjektstelle von $s^{2}$ TS ${ }^{\text {mit einer Proform }}$ besetzt. die bei der Ableituna der Oberflüächenstrouktuty $x_{20}$ 
getilgt wird (s. im Unterschied dazu die temporale Prädikation mit NP verb unten S. 146).

Die Verwendung von $\mathrm{s}^{2}$ TS als temporale Prädikation der Klasse Advb Temp loc legt die satzsemantischen Relationen fest: Adv Temp loc prädiziert über Argumente der Klasşen "Ereignis" und "Vorgang". Tiefenstrukturell illustriert $s^{3}$ TS diese Verhältnisse: die subjektstelle ist mit $^{l}{ }^{l}$ ns nach einer Nominalisierungs-TF, der erwähnten NP ${ }^{V P}$, besetzt. Linguistische Ergebnisse, gewonnen an konkreten Sätzen, entsprechen damit prädikatenlogischen Untersuchungen: das Temporaladverbial bestimmt das konjugierte Prädikat näher; damit ist seine Verwendung und sein Bezugsbereich im Unterschied zu den lokalen Adverbialen limitiert.

Die metasprachliche Formulierung des finiten Prädikats von $\mathrm{S}_{\text {TS }}$ als Argument des zugrundegelegten Satzes $\mathrm{S}^{3}{ }_{\text {TS }}$ ist bei einer Vielzahl von Sätzen aufgrund der objektsprachlichen Existenz ${ }_{17}$ des entsprechenden Lexems als Nomen unproblematisch. ${ }^{17}$ In der Tat kann man die Feststellung machen, daß $\mathrm{s}^{3}$ TS formal und funktional als vergleichbare struktur von Oberflächensätzen wie $z u(8-16)$ fungieren kann (s. dazu noch unten 2). Hier soll darauf hingewiesen werden, daß auch bei nicht vorhandenen oder dem Verb semantisch nicht entsprechenden nominalisierten Formen des finiten Prädikats der Prädikations-Test die Beziehung der Advb Temp ${ }_{\text {loc }} \mathrm{z}$ dem übrigen Satz deutlich macht. Dazu nur zwei Beispiele zur Illustration, da sonst erforderliche wortbildungsregeln und Bedeutungsdifferenzierungen zwischen den wortklassen hier nicht behandelt werden können.

(17) No v trudnuju minutu uXenyj ne rasterjalsja.

$\mathrm{S}_{\mathrm{TS}}$ : UCenyj ne rasterjalsja.

$\mathrm{s}_{\mathrm{TS}}^{3}$ : To obstojatel'stvo, ¿to uðenyj ne rasterjalsja, imelo mesto $v$ trudnuju minutu.' 
$\mathrm{V}$ sadu živet solovej. Ego pervye večernie zvuki razdalis' $v$ minutu vseobక̌̌ego molčanija.

Ein Nomen der hier verwendeten Bedeutung von "razdat'sja" verzeichnen die wörterbücher nicht. "Razdač" ist die nominalisierte Form von "razdat" in der Bedeutung von "otdat', raspredelit' meždu vsemi" (Slovar' (AN) (1950 ff) Bd. 12, Sp. 269). Dennoch sind auch für (18) die Ergebnisse des Prädikations-Tests plausibel; man vgl.:

STS : Razdalis' ego pervye zvuki.

$s_{\text {TS }}^{3}$ : To sobytie, tto razdalis' ego pervye zvuki, imelo mesto $v$ minutu vseobక̌čgo molčanija.

2. Als metasprachliche Formulierung des temporalen Prädikats von $S$ TS habe ich mich für "imet' mesto" entschieden. Ich stütze mich dabei auf die referierten Ergebnisse von Krause und weise darauf hin, daß hier "imet' mesto" als generalisierte proform im Rahmen des temporalen Prädikats verstanden wird. In Oberflächensätzen finden wir im Russischen - ähnlich wie im Deutschen - eine Reihe weiterer Verben als proformen in temporalen Prädikaten, die unterschiedliche wortsemantische Merkmalstrukturen aufweisen. Ich komme damit zu der Bemerkung von oben zurück, daß $S^{3}$ TS des Prädikations-Tests Oberflächensätzen syntaktisch und funktional entsprechen kann. Man vgl.:

(19) Kak naročno; $v$ sledujuకčuju minutu s nim slučilos' neprijatnoe proiš̌estvie: on ostupilsja.

In dem Text aus Dostoevskijs "Djadjuškin Son" wird das finite Prädikat - "ostupilsja" - in einer semantisch generalisierten und syntaktisch nominalisierten Form als "proisšestvie" vorweggenommen. Uber dieses Nomen wird das temporale Prädikat: "slučilos' v sledujuš̌uju minutu" ausgesagt. 
3. Die Analysen geben uns auch gewisse Aufschlüsse über die Funktion der Präposition. Diese leitet sich von der Funktion der temporalen prädikation ab, als deren Bestandteil die Präposition fungiert. Bei PP temp mit NP zeit regeln die Präpositionen zum einen die Opposition Advb Temp zu Advb Temp quant (s. oben (3) und (4)). Zum anderen ordnen sie innerhalb der Klasse Advb Temp loc durch ihre lexikalischen Oppositionen wie auch deren Neutralisierung ("do", "posle", "v", etc.) das System der zeitlichen Relationen in der Sprache (s. Beispiele dazu unten S. $142 \mathrm{f}$ ).

Bei PP temp mit NP zeit ist die bekannte Mehrdeutigkeit einer Reihe von präpositionen nicht relevant, da ihre Bedeutung maßgeblich durch die lexikalische Besetzung der NP bestimnt ist. Anders sind die verhältnisse bei PP temp mit NP verb ' worauf ich unten (S. $144 \mathrm{ff}$ ) zu sprechen komme.

4. In den Beispielen (8-19) stehen die Advb Temp an beliebigen Stellen des Satzes. Im Unterschied zu den lockalen Adverbialen stellen wir keine Mehrdeutigkeit der Prädikationsmöglichkeiten fest. Das ist im zusammenhang damit zu sehen, daß die lokalisierend temporalen Adverbiale in der Regel die finiten Prädikate bzw. deren nominalisierte Entsprechungen oder Proformen näher bestimmen - Mehrdeutigkeit kann in Sätzen mit mehr als einem finiten Prädikat entstehen (s.u. S. 166 ff). Für die bisher untersuchte Struktur von Oberflächensätzen mit Advb Temp loc bei NP zeit gilt, daß die semantische zuordnung festgelegt ist und es keiner weiteren Motivation - wie etwa der T-C-A bei den Lokaladverbialen Kap. 3.2, S. $86 \mathrm{ff}$ ) - zur Disambiguierung der syntaktisch "losen" Erweiterung von Sätzen mit Advb Temp loc bedarf. Das heist natürlich nicht, daß nicht auch für die verschiedenen Positionen der Advb Temp loc die Regeln der T-C-A gültig sind, jedoch mit dem Unterschied, daB funktionale Beziehungen in der Regel nicht zur Beschreibung und Auflösung von satzsemantischen Mehrdeutigkeiten herangezogen werden müssen. 
Die bisherigen Ergebnisse über die TS von Sätzen mit Advb Temp lassen sich in folgendem Phrasenstrukturbaum darstellen:

Schema 3a

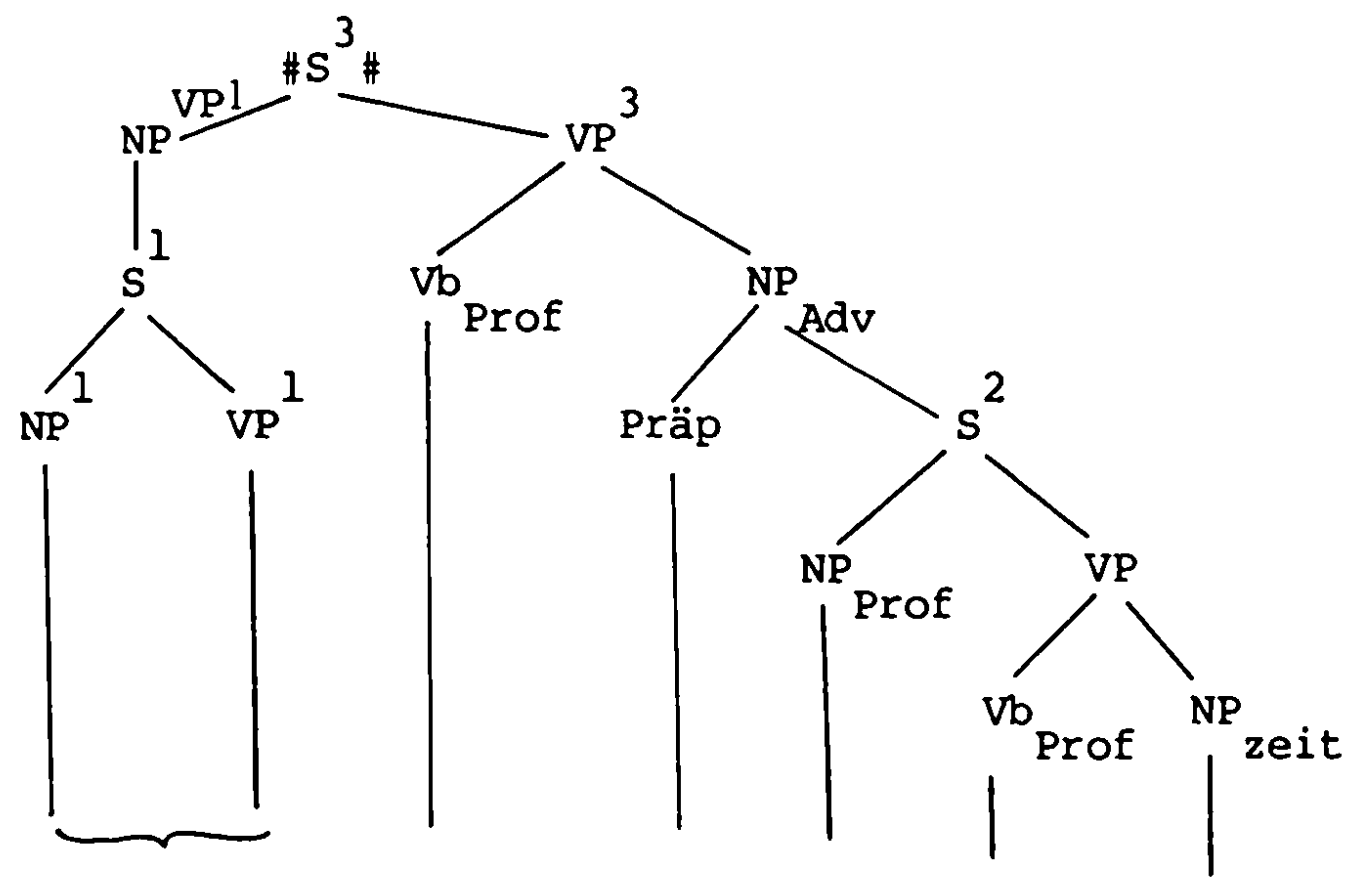

Podn. M. v vozduch im.mesto $v \quad \theta \quad \theta$ tot pamj. den'. $\left(S_{\text {TS }} \mathrm{zu}(8)\right)$

Eine Gegenüberstellung der Schemata l (S. 70) für die lokale Prädikation und $3 a$ für die temporale zeigt der Hierarchie der Sätze der $\mathrm{TS} S$ und $S$. Für die lokale Prädikation sind $s^{1}$ und $s^{2}$ Schwesterknoten. Damit soll die Linearität der Beziehung von zwei und mehr Sätzen, die durch lokale Prädikationen miteinander verbunden sind, zum Ausdruck gebracht werden (vgl. dazu auch die Ausführungen zum Satzkonnektor von $\mathrm{s}^{1}$ TS und $\mathrm{s}^{2} \mathrm{TS}$, $\left.\mathrm{s} .71 \mathrm{ff}\right)$.

Eine weitere Bestätigung für die dort getroffene Entscheidung der schematischen Darstellung der Ableitungsgeschichte von Sätzen mit PP loc geben die Analysen unter funktionalem Gesichtspunkt be ${ }^{10 C} \mathrm{PP}$ in Anfangsstellung: aus diesen konnte gefolgert werden, $\mathbb{d a \beta}_{\mathrm{B}}^{\mathrm{l}}$ Existentialsätze und Sätze mit lokaler Prädikation komplementäre Prädikationen sind (S. $118 \mathrm{ff}$ ), die damit austauschbare Rollen bei der strukturierung von Sätzen 
übernehmen können. Entgegen der linearen ordnung sprachlicher Ausdrücke durch lokale Prädikation impliziert die temporale Prädikation eine hierarchische Ordnung. ${ }^{20}$ Die Beobachtungen zur Satzsemantik wie auch zu formalen Charakteristika, die dafür sprechen, die Derivationsgeschichte von Sätzen mit PP temp so zu modellieren, daß $S^{2}$ der TS $s^{1}$ übergeordnet ist, sollim Zusammenhang mit $\mathrm{PP}_{\text {temp }}$ bei $\mathrm{NP}_{\text {verb }}$ weiter erörtert werden.

Zunächst soll noch - in Ergänzung zu (8) - (19) - die Frage angeschnitten werden, welche Folgen die Substitution der Präposition innerhalb der PP temp für die satzsemantischen Verhältnisse hat. Die Analyse der folgenden Beipiele zeigt, das sich mit Schema $3 a$ allgemein die syntaktischen Beziehungen von Advb Temp loc im Satz beschreiben lassen, unabhängig davon, mit welcher Präposition die PP temp besetzt ist.

In den Sätzen (8) - (19) bezeichnet die Präposition "v" (bei anderen Lexemen von NP, ist es "na") den "Zeitpunkt" oder den "Zeitraum", durch den das "Ereignis" oder der "Vorgang" näher - eben temporal - bestimmt wird. Es liegen Untersuchungen zur Topologie der zeitbezeichnungen im Russischen vor; der allgemeine Verweis auf eine neuere Darstellung in: Grammatika (Praha) (1979), Bd. II, S. 767 - 769, soll genügen. Für die Fragestellung hier ist relevant, daß wir Schema 3a entsprechende TS bei PP mit z.B. den Präpositionen "Kerez, pered, posle" etc. erhalten. Man vgl.:

(20) On uxilsja govorit' i pisat' po-anglijski i Cerez neskol'ko let on daže pisal na ètom inostrannom dlja nego jazyke stichi.

$\mathrm{S}_{\mathrm{TS}}^{1}$ : On pisal na ètom jazyke stichi.

$\mathrm{s}_{\mathrm{TS}}^{3}$ : Pisanie stichov ... imelo mesto đerez neskol'ko let. 
(21) Official'no semestr nacalsja 1-ogo oktjabrja, a student Ivanov vozvraščalsja tol'ko čerez 2 nedeli.

$\mathrm{S}_{\mathrm{TS}}^{1}$ : Student Ivanov vozvraščalsja.

$\mathrm{S}_{\mathrm{TS}}^{3}$ : Vozvraščenie studenta Ivanova imelo mesto čerez 2 nedeli.

(22) Na sobranii ... on kak vsegda zastupilsja za predloženie rukovodstva ob''edinenija, odnako čerez den' $v$ krugu druzej on vyskazyvalsja ob etom proekte dovol'no otricatel'no.

$\mathrm{S}_{\mathrm{TS}}$ : On govoril ob ètom proekte dovol'no otricatel'no.

$\mathrm{s}^{3} \mathrm{TS}:$ Ego vyskazyvanie ob étom proekte imelo mesto ¿erez den'...

(23) Vsjakij ... pomnit minutu, kogda zal zamiral pered načalom uvertjury baleta.

$S^{1}$ TS : zal zamiral.

$\mathrm{s}_{\mathrm{TS}}^{3}$ : To, cto zal zamiral, imelo mesto pered načalom uvertjury baleta.

(24) Pered Novym Godom my vstretilis' u Lizy.

$\mathrm{s}_{\mathrm{TS}}^{1}$ : My vstretilis' u Lizy.

$\mathrm{s}_{\mathrm{TS}}^{3}$ : Naša vstreca ... imela mesto pered Novym Godom.

(25) Lemm posle čja sygral emu svoju kantatu.

$\mathrm{S}_{\mathrm{TS}}^{1}$ : Lemm sygral emu svoju kantatu.

$\mathrm{s}_{\mathrm{TS}}^{3}$ : Igra kantaty L. imela mesto posle čaja. 
4.1.2 ABLEITUNG DER TS VON $\mathrm{S}_{\mathrm{OF}} \longrightarrow \mathrm{NP}^{1} \mathrm{VP}^{1}(\mathrm{X}) \mathrm{PP}_{\text {temp }}(2)$

Bei Temporaladverbialen kann das NP der PP mit einem Nomen besetzt sein, das von einem verb abgeleitet ist oder in loser Wortbildungsrelation $z u$ dem entsprechenden verbalen Lexem steht. Von den hier $z u$ behandelnden Fällen liegt mindestens eine teilweise semantische Merkmalentsprechung zwischen Nomen und Verb vor. Man vgl.:

(26) V besedach s ucenikami professora Bloka, pri Čtenii knigi Spektorskogo "Aleksandr L'vovit Blok" poet vse glubže osoznaval to, o そem napisal materi.

Bei den PP handelt es sich um PP temp der Klasse Advb Temp ${ }_{\text {loc }}$ Die Uberprüfung ihrer Relationen $z$ u den übrigen Satzgliedern mit Hilfe des Prädikations-Tests ergibt:

$\mathrm{S}_{\mathrm{TS}}^{1}$ : Poèt vse glubそ̌e osoznaval to, o ไem napisal materi. $\mathrm{s}_{\mathrm{TS}}^{2}$ : Poét besedoval s uX̌enikami, čital knigi ...

$\mathrm{S}_{\mathrm{TS}}^{3}$ : Osoznanie poètom togo, o tem on napisal materi, imelo mesto $\mathrm{v}$ besedach $s$ uěnikami Prof. Bloka $i$ pri ctenii ...

Man kann feststellen, daß die tiefenstrukturellen Relationen der PP temp von (26) zu den übrigen Satzgliedern unseren Analysen des Temporaladverbials mit NP entsprechen: das konjugierte Prädikat des Oberflächensatzes erhält durch die PP eine nähere temporale Bestimmung. Auf der Ebene der TS ist das konjugierte Prädikat Argument der temporalen Prädikation "v besedach", "pri Ztenii", s. S $\mathrm{S}^{3}$. Anstelle einer Bezeich-

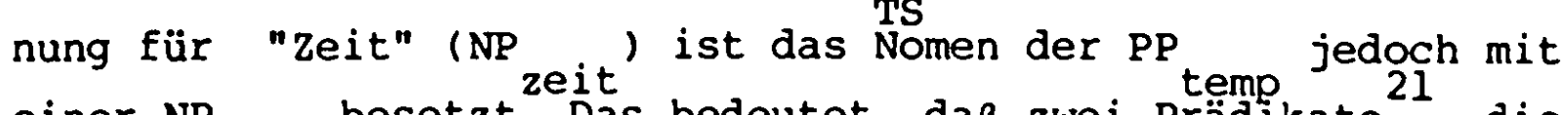
einer NP verb besetzt. Das bedeutet, daß zwei Prädikate 21 , die lexikalisch einer bzw. voneinander abgeleiteten Kategorien angehören, in Relation zueinander gesetzt werden. Dies führt zu sprachlichen Spezifika, die die Sätze mit NP zeit innerhalb 
der PP temp nicht aufweisen. Bevor ich $z u$ der Darstellung dieser strukturellen Eigenschaften komme, sollen zur Illustration noch einige Belege mit PP temp' wobei NP verb ist, zitiert werden:

(27) Vo vremja našego vozvraక̌̌enija domoj my razgovarivali ticho.

St ${ }_{\text {TS }}$ : My razgovarivali ticho.

S2 2 : My vozvrascalis' domoj.

$\mathrm{S}_{\mathrm{TS}}^{3}$ : NaS razgovor imel mesto vo vremja nasego vozvrašenija domoj.

(28) A romantiðeskie i geroið̌eskie meðty moi sovsem vyleteli iz golovy pri pervom stolknovenii s dejstvitel'nost'ju.

$\mathrm{S}_{\mathrm{TS}}^{1}$ : A romantiðeskie i geroiðeskie mexty moi sovsem vyleteli iz golovy.

$\mathrm{s}_{\mathrm{TS}}^{2}$ : Moi mexty vpervye stolknulis' s dejstvitel'nost'ju.

$\mathrm{S}^{3} \mathrm{TS}:$ To $\left\{\begin{array}{l}\text { obstojatel'stvo } \\ \text { sobytie }\end{array}\right\}$, cto romantiCeskie i

geroiðeskie mexty vyleteli iz golovy, imelo mesto pri pervom stolknovenii s dejstvitel'nost'ju.

(29) Pri vide milicionerov prijatel' Gladkova $\left\{\begin{array}{l}\text { pustilsja nautek. } \\ \text { pobezal. }\end{array}\right\}$

$\mathrm{S}_{\mathrm{TS}}^{\mathrm{l}}$ : Prijatel' Gladkova $\left\{\begin{array}{l}\text { pustilsja nautek. } \\ \text { pobezal. }\end{array}\right\}$

$\mathrm{s}^{2} \mathrm{TS}$ : Prijatel' Gladkova uvidel milicionerov.

$\mathrm{S}^{3} \mathrm{TS}$ : To sobytie, Cto prijatel' Gladkova

$\left\{\begin{array}{l}\text { pustilsja nautek } \\ \text { pobezal }\end{array}\right\}$ imelo mesto pri vide milicionerov.

(30) Pri izučenii semejnoj kul'turnoj tradicii ... ego biografy mnogo pisali o ... sem'e materi poèta.

$\mathrm{S}_{\mathrm{TS}}^{l}$ : Ego biografy mnogo pisali o sem'e materi poeta. 
$S^{2}$ TS : Ego biografy izuCali semejnuju kul'turnuju tradiciju. $\mathrm{s}_{\mathrm{TS}}$ : Pisanie ego biografov o sem'e materi poèta imelo mesto pri izučenii semejnoj kul'turnoj tradicii.

Schema 3a eignet sich auch für eine modellhafte Darstellung der TS der Sätze vom Typ (26-30). Dabei sind die Knoten, dominiert von $\mathrm{s}^{2}$, anstelle der Proformen mit ihren kategorialen variablen ${ }^{22} \mathrm{zu}$ besetzen; das berücksichtigt Schema $3 b$.

Schema $3 b$

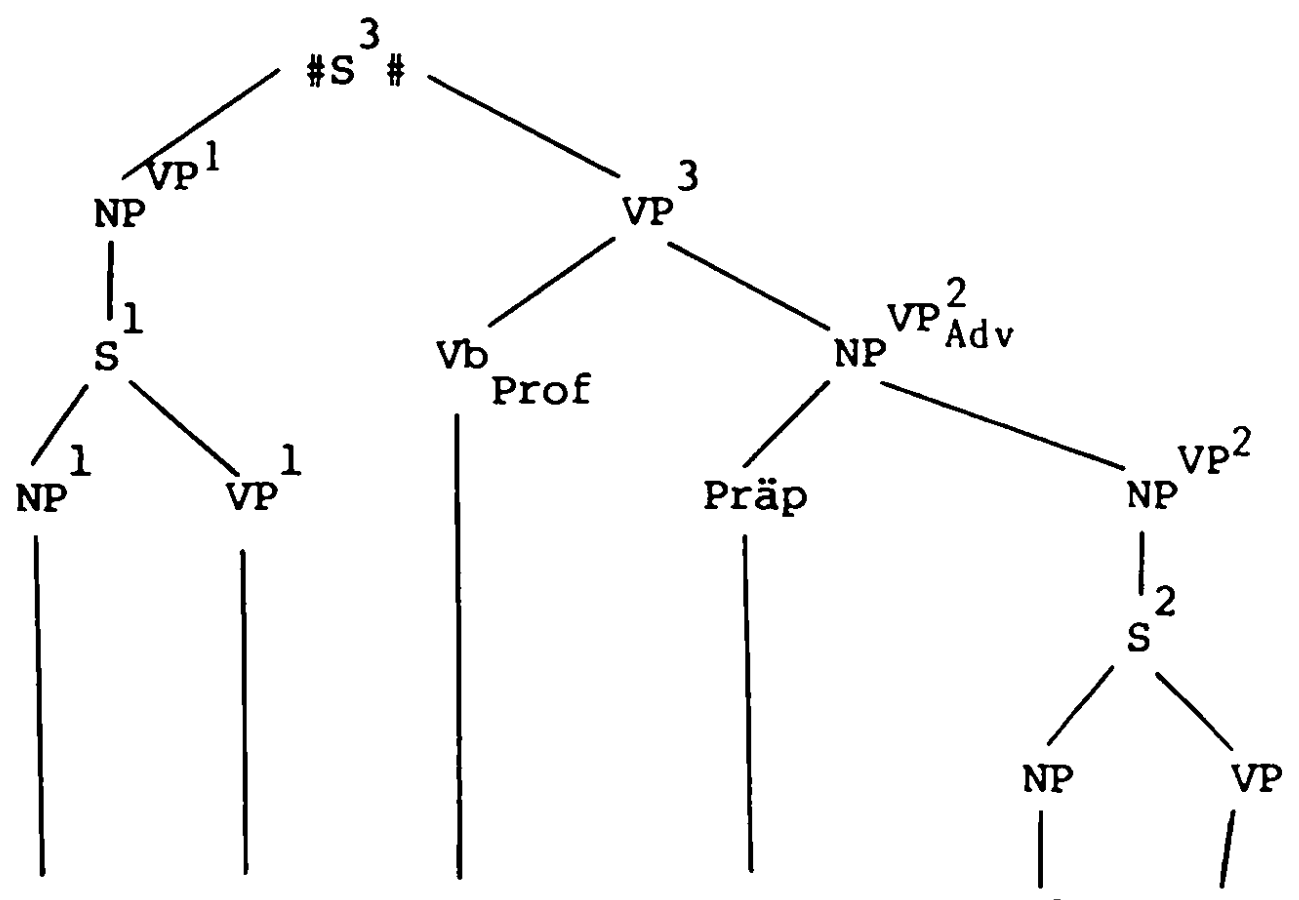

Naš razgovor imel mesto vo vremja naš. vozv. domoj. (S $\left.{ }^{\mathrm{zu}}(27)\right)$

Für die Darstellung der Sätze mit PP ${ }_{\text {temp }}$ bei NP ${ }_{\text {verb }}$ ist es unumgänglich, dem Problem der Mehrdeutigkeit ein Kapitel zu widmen (s. unten S. $166 \mathrm{ff}$ ). Es sei in diesem Zusammenhang hier nur - mit Hinweis auf das Schema - auf den Unterschied $2 w i s c h e n 3 a$ und $3 b$ hingewiesen, der für die Funktion der Prāposition bei NP ${ }_{\text {verb }} z u$ erwarten ist: die Präposition gewinnt für die Festlegung der Relation zwischen $S^{2}$ und $S^{1}$ als eine temporale größere Relevanz. Diese Funktion erfüllt bei Advb Temp ${ }_{\text {loc }}$ mit $\mathrm{NP}_{\text {zeit }}$ im wesentlichen die 
Zeitbestimmung. Steht anstelle von $\mathrm{NP}_{\text {zeit }}$ nun $\mathrm{NP}_{\text {verb }}$ sind im Prinzip verschiedene Prädikationsverhältnisse zwischen $s^{2}$ TS und $s^{1}$ offen.

Die Präpositionen sind m.E. bei dieser Konstellation satzsemantischer Verhältnisse und deren Ausdruck durch sprachliche Formen der einzige formale Faktor, der die wahl des $\operatorname{im}_{2}$ gegebenen Satz intendierten Prädikationsverhältnisses von $S^{2}$ TS $24 S^{1}$ festlegt. In diesem Zusammenhang läßt sich dann beschreiben, daß die bekannte lexikalische Mehrdeutigkeit vieler Präpositionen einer der Faktoren der zu beobachtenden Mehrdeutigkeit in Sätzen mit PP ist, wenn die Stelle des Nomens mit NP verb besetzt ist.

Hier sollen zunächst einige Beobachtungen $\mathbf{z u}$ den strukturellen Möglichkeiten, die die Sätze mit ${ }_{\text {verb }}^{\text {in der } P P_{\text {temp }}}$ aufweisen, zur Sprache kommen.

Mit den Sätzen (26-30) wurden Beispiele angeführt, in denen die NP von $S^{1}$ und $S^{2}$ TS identisch sind und im Oberflächensatz - z.B. als Attribute - explizit bleiben, vgl.: "My razgovarivali vo vremja našego vozvrašenija domoj".

Daneben gibt es Sätze, in denen zum einen die besagten NP nicht identisch sind, zum anderen infolge von weiteren $T f$, wie z.B. der Passiv-Tf, getilgt sind.

Bei der Analyse der folgenden Beispiele für nicht identische NP von $s^{1}$ und $s^{2}$ TS ist die NP von $s_{T S}^{1}$ mit "__ " und die von $s^{2}$ TS mit " $========="$ hervorgehoben, soweit sie im Oberflächensatz lexikalisch besetzt sind. Der PrädikationsTest wird nur an einem Beispiel exemplarisch durchgeführt:

(31) V samyj razgar ètoj oglušitel'noj potechi $k$ vorotam pod' 'echal zagrjaznennyj tarantas.

$S_{\text {TS }}^{1}: K$ vorotam pod' 'echal zagrjaznennyj tarantas. 
$S_{T S}^{2}$ : Razgorelas' oglusitel'naja potecha. 25

$\mathrm{S}_{\mathrm{TS}}^{3}$ : Pod''ezd zagrjaznennogo tarantasa imel mesto $v$ samyj razgar étoj oglusitel'noj potechi.

(32) ... vsjakij v ee prisutstvii totčas čuvstvoval sebja kak by doma.

(33) ... (mat') pri vsjakom ego valete protjagivala ruki k ... ego nožkam.

(34) (... on grozit tebe rasprostranit' po gorodu tvoi pis'ma.) Pri ètoju ugroze ty vychodiš' iz sebja.

(35) ... kotoromu on pri žizni okazyval svoe vysokoe pokrovitel'stvo.

Die Belege (34) und (35) machen auf die Folgen im Oberflächensatz bei nicht identischen NP von $S$ TS und $S^{2}$ TS aufmerksam. Während (31) - (33) die Möglichkeiten "ökonomischer" Information in der Sprache illustrieren, geben (34) und (35) Beispiele für Informationsverlust nach mehrfacher Tf, wie sie die Ableitung dieser Sätze modelliert: in (34) ist der Rückgriff auf den weiteren Kontext notwendig, um bestimmen zu können, daß das Demonstrativpronomen "éta" hier in der Funktion einer Proform für die NP von $S^{2}$ TS steht. In (35) ist die NP von $\mathrm{s}^{2}$ fS formal nicht explizit. ES kann daher nur aus dem weiteren Kontext geschlossen werden, auf welche NP sich "pri žizni" bezieht - diese kann identisch sein mit $\mathrm{NP}^{1}$ von $\mathrm{S}^{1} \mathrm{TS}^{\prime}$ jedoch nicht unbedingt. So z.B. in:

(35a) Nikolaj byl $v$ kontakte s Puskinym, kotoromu on pri そ̌izni okazyval svoe vysokoe pokrovitel'stvo.

Der Informationsverlust infolge von Tilgung der NP ist nicht auf $s^{2}$ bs beschränkt, sondern läßt sich auch für $s^{1}$ ts beob- 
achten: die NP ist z.B. in der Regel nach Passiv-Tf getilgt. Man vgl.:

(36) Na podpisanii soglašenija prisutstvovali takže sekretari pravlenija SP SSSR.

(37) V chode besedy byli obsuždeny voprosy razvitija sovetsko-norvežskich otnoక̌enij ...

(38) Posledovatel'nyj perevod ispol'zuetsja vo vremja peregovorov, pri soprovoźdenii delegacij ...

(39) Sokraščennyj rabočij den' možet ustanavlivat'sja dlja zenštin s det'mi ... ne tol'ko pri postuplenii na rabotu no i ...

(40) Bjuro krajkoma objazalo ... obkom opredelit' četkie kriterii ... pri zanesenii imen peredovikov na dosku i $v$ knigu počta.

(40) ist ein Beispiel für die hier behandelte Oberflächenstruktur nach einer, weiteren Tf, der subjekthebung. ${ }^{26}$ So ist in $(40) \mathrm{NP}^{1}$ von $\mathrm{S}^{2}$ objekt eines weiteren übergeordneten Satzes "Bjuro objazalo NP." 


\subsection{ZUM PROBLEM DER HIERARCHIE DER PRÄDIKATIONEN IN SÄTZEN MIT TEMPORALADVERBIALEN}

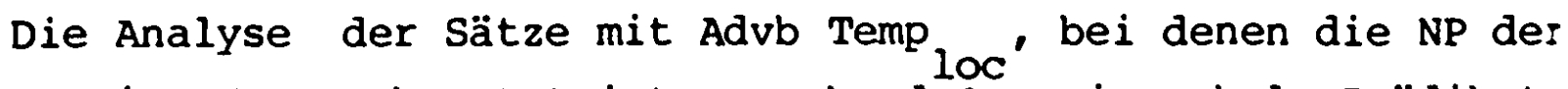
PP mit NP verb besetzt ist, ergab, daß zwei verbale Prädikate zueinander in Relation gesetzt werden. Von objektsprachlichen Formen stehen für den Ausdruck dieses Verhältnisses z.B. die nominalisierte Form eines finiten Verbs (VP) und die PP des anderen zur verfügung. NPVPl ist damit subjekt $z u$ den Prädikat $\mathrm{Vb}_{\text {Prof }}{ }^{\mathrm{PP}}$ temp

Mögliche Oberflächenstrukturen sind:

- Razgovor imel mesto pri našem vozvrašenii domoj 27

- My razgovarivali pri (našem) vozvrašcenii domoj.

Diese Sätze werfen die Frage nach der Hierarchie der Prädikate, die hier in eine temporale Relation gebracht werden, auf.

In der Literatur ist diese Frage im Zusammenhang mit Partizipialkonstruktionen behandelt worden.

D. Weiss übernimnt bei seiner Darstellung polnischer Adverbialpartizipien den von R. Rủžička (1966) geprägten Begriff der "sekundären Prädikation" oder "backgrounding" (Weiss (1978), 254). Jedoch sein Versuch, mit diesem Begriff die unterschiedlichen Verwendungsmöglichkeiten von Partizipialkonstruktionen im Polnischen $z u$ beschreiben, führt 28 nach seinen eigenen Worten $z u$ widersprüchlichen Ergebnissen.

Zum einen läßt sich aus der Gegenüberstellung: Hauptsatzgefüge und Satz mit Adverbialpartizip das wichtige Argument gewinnen, daß diese Konstruktionen auf verschiedene TS zurückzuführen sind, wobei für die TS des Satzes mit Adverbialpartizip entscheidend ist, daß die Prädikate in einer hierarchischen Relation zueinander stehen. 
Das zeigen im besonderen solche Satzpaare, für die die Tf des Satzes mit Adverbialpartizip in ein Hauptsatzgefüge blockiert ist oder bei denen ein Satz mit anderer Bedeutung entsteht, man vgl.:

(10a) Staruszek szedł czXapiąc do biurka.

(10b) *Staruszek szedł do biurka i czlapał. (Weiss, loc. cit., 258)

zu der hierarchischen ordnung der Prädikate in Sätzen wie (10a) gibt Weiss folgenden Kommentar:

"Es gibt also offensichtlich Konstellationen von Sachverhalten, die sich einer wiedergabe durch Hauptsatzgefüge widersetzen, ... . Woran liegt das? zumeist offenbar daran, das der eine Sachverhalt als nicht gleichwertig, eben 'sekundär' (vgl. 'sekundäre Prädikation') gegenüber dem anderen erscheint, sei es nun, daß er diesen nur präzisiert, sei es umgekehrt, daß er mit ihm überhaupt keinen zusammenhang hat. Im Gegensatz zur Koordination mit 'und', die die beiden verbundenen Glieder auf dieselbe Ebene stellt, leistet die Partizipialisierung genau diese gewünschte kommunikative Hierarchisierung: nicht-restriktive Attribute bzw. sekundäre Prädikate erscheinen gegenüber der Hauptprädikation rangmäßig zurückgestuft, in den Hintergrund gedrängt ..." (ibid).

Diese Beobachtungen stehen im Widerspruch zu anderen Sätzen. "Einen Sonderfall stellen eingliedrige (ev. auch mit adverbialen Ergänzungen versehene) Partizipialphrasen dar, die dem Hauptprädikat folgen: in dieser stellung können sie an die Spitze der kommunikativen Hierarchie aufrücken, zum 'Fokus' werden, weisen jedenfalls auf der Rangskala einen höheren Wert auf als die Hauptprädikation, vgl. ' $X$ szedX CZEAPIA̧C', ' $X$ wykładał STOJA̧C', ' $X$ przegląał gazety LEŻA̧C', usw. Entgegen dem sonstigen 'Hintergrundcharakter' der partizipialen Konstruktion sind solche Partizipien sogar 
prädestiniert für die höherwertige Information ..." (loc. cit., 260).

Ich habe die aufschlubreiche Untersuchung von weiss ausführlich referiert, weil die Analysen zu PP, im besonderen die hier zur Diskussion stehende Klasse Advb Temp ${ }_{\text {loc }}$ bei NP verb' sprachliche Fakten enthalten, die entsprechende Probleme aufwerfen. Man kann nicht ausschließen, daß die unterschiedlichen Oberflächenformen - einerseits Adverbialpartizip, andererseits nominalisiertes Verb im Rahmen einer PP - auch spezifische sprachliche Funktionen beinhalten. Dennoch scheint es mir möglich, vom Standpunkt der Analysen der PP die zusammenhänge, die $z u$ den oben dargestellten widersprüchlichen Ergebnissen über die "sekundäre Prädikation" geführt haben, aufzudecken.

Weiss verwendet $^{29}$ für seine Untersuchungen den Rollentausch von finitem verb und partizipialer Konstruktion. Getauscht werden hierbei die grammatische Funktion von finitem verb und partizipialer Konstruktion (Es gibt Argumente dafür, daß man "Rollentausch" für das anstehende Problem auch noch anders durchführen kann oder muß, s. dazu unten).

Der Test nach weiss sieht folgendermaßen aus:
"(12a) Leżąc na łóżku, przeglądał gazety.
(12a) Leżał na łóżku, przeglądając gazety " (Weiss, loc. cit., 259).

Die Möglichkeit des Rollentauschs kommentiert weiss mit den Worten: "AuBerdem dürften zahlenmäßig die Fälle überwiegen, wo dem Sprecher freie wahl überlassen bleibt bei der Plazierung des 'backgrounding' "(ibid.).

Ist die Feststellung zutreffend, daß "dem Sprecher freie wahl überlassen bleibt bei der Plazierung des "backgrounding"? Für die Beantwortung dieser Frage übertrage ich die Problemstellung auf Sätze mit Advb Temp ${ }_{1 m}$; es sollen zunächst nur 
solche Beispiele zur Sprache kommen, deren PP eindeutig temporal $^{30}$ ist.

Der Test wird insofern verändert, als der grammatische Rollentausch von finitem Verb und PP durch einen Rollentausch der Glieder der aktuellen Satzperspektive ergänzt wird. Diese Ergänzung scheint mir geeignet zu sein, das gestellte problem deutlich $2 u$ machen. Der Rollentausch wird in zweifacher Hinsicht durchgeführt. Zur Illustration möge folgendes Beispielset ${ }^{31}$ dienen:

$\left.3\left\{\begin{array}{l}\text { (41) On kurit pri molitve. } \\ \text { (42) } \text { Pri kurenii on molitsja. }\end{array}\right\} \begin{array}{l}1 \\ \text { (43) } \text { On molitsja pri kurenii. }\end{array}\right\}$

Ein Vergleich der Formen dieser Sätze ergibt, daß sie in dreifacher Relation zueinander stehen:

1. (41) und (42) unterscheiden sich oberflächenstrukturell durch den grammatischen Rollentausch von finitem verb und Advb Temp $10{ }^{\circ}$

2. (42) und (43) unterscheiden sich in der wortfolge ihrer Glieder in der Weise, daß deren Positionen von Thema und Rhema vertauscht sind.

3. (41) und (43) unterscheiden sich in zweifacher Hinsicht: durch den grammatischen Rollentausch (wie bei 1) und durch die Gliederung der Aussage (wie bei 2 ).

Kommen wir zu unserer oben gestellten Frage zurück: hat der Sprecher bei der Plazierung des "backgrounding" freie Wahl? Die Antwort dürfte "nein" lauten - die Plazierung des "backgrounding" ist nicht der freien Wahl des Sprechers überlassen, sondern je nach seiner wahl sind damit verschiedene Bedeutungen der Sätze verbunden. Worin bestehen nun diese Unterschiede?

1. (41) und (42) sind einem jesuitischen witz ${ }^{32}$ nachkonstruiert, dessen witzigkeit auf ihrer - nur sprachlich? gegensätzlichen Bedeutung aufgebaut ist: (4l) ist gemäß der 
Ordensregel streng verboten; da bleibt aber doch (42), wogegen es keine Instruktionen gibt.

2. (42) und (43) unterscheiden sich eindeutig nicht in ihrer referentiellen Bedeutung: für (43) gilt das für (42) Gesagte. Ein Bedeutungsunterschied resultiert dagegen aus der unterschiedlichen Bewertung aufgrund der veränderten kommunikativen Bedeutung ${ }^{33}$ der Satzglieder, die Thema- und Rhemapositionen besetzen.

3. (41) und (43) unterscheiden sich einerseits wie (41) und (42), und auBerdem wie (42) und (43). Deshalb dürften diese beiden Sätze als Gegensatzpaar der hier gemachten Aussagen verstanden werden und nicht (41) und (42).

Da die Bedeutungsdifferenzierung von (42) und (43) im Russischen für expressiv neutrale Aussagen generell gilt, beschränke ich mich im Folgenden auf die Beschreibung des Bedeutungsunterschieds von (41) und (42).

Um den Bedeutungsunterschied von (41) und (42) linguistisch $\mathrm{zu}$ beschreiben, fragen wir nach der Relation zwischen den beiden Prädikationen im Satz. Die Untersuchungen zu Beispielsets wie (41) - (43), s. auch im folgenden (44) (52), machen die TS, wie sie Schema 3b skizziert, plausibel.

Das Verhältnis der beiden in Beziehung gesetzten Prädikate ist hierarchisch, und das unabhängig davon, in welcher der zwei uns interessierenden oberflächenstrukturen: finites Prädikat und PP oder nominalisierte Form des verbs und Prädikat, bestehend aus (Proverb PP temp' - die Sätze erscheinen. Die Hierarchie sieht dabei so aus, daß die Prädikation in der Form der PP die übergeordnete ist. Damit findet unser intuitives Verständnis des Unterschieds von z.B. (41) $2 u$ (42) eine Erklärung, das beinhaltet, daß das "Geschehen", das mit den PP genannt ist, das "Hauptgeschehen" ist. Dieses Verständnis mag der Vorstellung von der Syntax der Oberflächenstruktur des Satzes, in der das finite Verb als prädikativer Kern dominiert, widersprechen 
Prädikatenlogische Untersuchungen im besonderen an Sätzen mit PP Advb liefern m.E. auch für linguistische Untersuchungen umsetzbare Argumente einer notwendigen Unterscheidung zwischen Oberflächensyntax (mit z.B. dem finiten Prädikat mit Tempus und Modus) und Bedeutungsrelationen für den Aufbau der Aussage.

Die Analyseergebnisse, daß die Prädikation, die in der Oberflächenstruktur als PP temp erscheint, die andere Prädikation, die nun Argument ist, bestimmt, bezieht sich auf die satzsemantischen Verhältnisse. Ihr widerspricht nicht, das der PP ein niedrigerer wert auf der kommunikativen Rangskala zukommen kann, als dem finiten Prädikat oder einem anderen Satzglied, z.B. in (42) im Unterschied zu (41).

Man vgl. $z u$ den Ausführungen noch folgende Beispiele (Wortbildungsregularitäten mit evtl. Einschränkungen z.B. der stilistischen Akzeptabilität bleiben unberücksichtigt):

(44) Ona Citaet pri vjazanii.

(45) Pri ztenii ona vjažet.

(46) Ona vjažet pri đtenii.

(30) Pri izuð̌enii semejnoj tradicii ... ego biografy mnogo pisali o sem'e materi poèta.

(47) Ego biografy izucali semejnuju ... tradiciju pri pisanii o sem'e materi poèta.

(48) Pri pisanii o sem'e materi poèta biografy izucali semejnuju :.. tradiciju.

(36) Na podpisanii soglašenija prisutstvovali takže sekretari pravlenija SP SSSR.

(49) Soglašenija byli podpisany v prisutstvii sekretarej ....

(50) $\mathrm{V}$ prisutstvii sekretarej ... byli podpisany soglasenija. 
(29) Pri vide milicionerov prijatel' Gladkova pobežal.

(51) Prijatel' Gladkova uvidel milicionerov (tol'ko) pri pobege.

(52) Pri pobege prijatel' Gladkova uvidel milicionerov.

Es ist klar, daß die Regeln, die für die aktuelle Satzperspektive zu beobachten sind, ein relativ unabhängiges system $^{37}$ darstellen (vgl. Relation von (42) $\mathrm{zu}$ (43)), Mit der Einbeziehung der satzsemantischen Verhältnisse und dem Nachweis ihrer Relevanz in sprachlichen Strukturen wird jedoch beschreibbar, wieso die PP "Fokus" der Aussage sein können. Für die Beurteilung der Hierarche von Prädikaten in Sätzen mit PP muß allerdings differenziert werden, um welche Art Prädikation es sich handelt. Die bisherige Analyse ergab, daß es hier Unterschiede $z$ wischen Lokaladverbialen und Temporaladverbialen gibt: Lokaladverbiale sind den prozessualen Aufbauprinzipien von Bedeutung in der Sprache zuzurechnen, dagegen gehören die temporalen $z u$ den hierarchischen.

Mir scheint, daß das Fehlen der den konjugierten Prädikaten eigenen Kategorien, wie Tempus, Modus, kein Argument gegen die hier vorgenommene Hierarchisierung ist, nämlich daß Advb Temp loc satzsemantisch dem finiten prädikat übergeordnet sind. Tatsächlich drücken die PP temp diese Kategorien nicht aus. Es ist jedoch auch nicht temp, daß ihre explizite Bedeutung diese als vom Hauptverb abgeleitet, einschliebt. Ich kann auf dieses Problem nur hinweisen. Man vgl.:

(53) Podvižnye igry luč̌e provodit' na vozduche pri utrennej i večernej progulke.

Wàhrend das verbale Prädikat modale Bedeutung hat, ist die Aussage, die die PP enthält, assertorisch. 
Mit dem hier entwickelten Erklärungszusammenhang für Advb Temp loc läßt sich ein weiteres Problem einordnen; ich meine die Nonsens-Bildungen, die man im besonderen dann erhält, wenn die Präposition auBer temporal auch konditional interpretiert werden kann (s. dazu u. S. 172 ff). Bei Anwendung des Rollentauschs erhalten wir unsinnige Sätze (s. (54), (55)).

(39) Sokraščennyj rabočij den' ustanavlivaetsja dlja ženščin s det'mi pri postuplenii na rabotu.

(54) «Ženščiny s det'mi postupajut na rabotu pri ustanovlenii sokraščennogo rabočego dnja.

(40) Obkom dolžen opredelit' Zetkie kriterii pri zanesenii imen peredovikov na dosku početa.

(55) *Obkom zanosit imena peredovikov pri opredelenii Łetkich kriterij.

(54) und (55) stellen ein Prädikat-Argument-Verhältnis her, das den Regeln für eine Verwendung dieser Ausdrücke in einem Prädikat-Argument-Verhältnis nicht entspricht.

Man vgl. dazu die nominalisierte Form von (54) und (55):

(56) *Postuplenie zenక̌čin $s$ det'mi na rabotu imeet mesto pri ustanovlenii socraščnnogo raboð̌ego dnja.

(57) «Zanesenie imen peredovikov na dosku połeta imeet mesto pri opredelenii četkich kriterij. 


\subsection{TEMPORALADVERBIAL UND VERBALASPEKT}

Sätze mit Temporaladverbial werfen die Frage nach der Beziehung zwischen dem Adverbial und dem Verbalaspekt auf. Die Sätze (8) - (25) machen auf folgende Zusammenhänge aufmerksam: In der Regel ist die Verwendung beider Verbalaspekte möglich; die damit implizierten aspektuellen Unterschiede scheinen in keine "Kollision" mit den Temporaladverbialen zu geraten.

Man vgl. zu oben (8) ff die Verwendung des jeweiligen anderen Verbalaspekts in den folgenden Sätzen:

(58) Chimik Mendeleev podnimalsja $v$ tot pamjatnyj den' $v$ vozduch na vozdušnom šare.

(59) My vstrecalis' s drugom detstva v vychodnoj den'.

(60) Ėtot fil'm vyšel na èkrany $v$ proslom mesjace.

(61) Ja vozvraščalsja iz poezdki $v$ poslednij den' kanikul.

(62) Éta statuètka byla sozdana neizvestnym masterom $v$ bronzovom veke.

(63) On otrical, a na vtoroj den' priznavalsja, čto perepisyvaetsja s Klavdiej Michajlovnoj.

(64) $\mathrm{V}$ detstve $i$ otročestve Blok napisal korotkie rasskazy $i$ stichi.

(65) Ved' imenno èti rebjata rasstrelivali na dnjach 300 amerikanskich plennych.

(66) Viktor pornogal mne $v$ ètot tjaželyj god.

(67) On uxilsja govorit' po-anglijski i Cerez neskol'ko let on daže napisal na étom inostrannom dlja nego jazyke stichi.

(68) Pered Novym Godom my vstrečalis' u Lizy.

(69) Lemm posle Caja igral emu svoju kantatu.

Man könnte darauf verweisen, daß in der Fachliteratur die Verwendungsmöglichkeit beider verbalaspekte in Sätzen mit Temporaladverbialen behandelt ist. So heist es in diesem 
Zusammenhang, daB der vollendete Aspekt sich auszeichnet durch "konkretno-faktiČeskoe značenie", der unvollendete durch "obక̌če-faktiðeskoe znałenie" (Rassudova (1968), 17 ff).

Auch Mehlig (1980) betrachtet in seinem Modell einer Hierarchie der Aspektmerkmale die Beziehungen zwischen Verbalaspekt und Temporaladverbial oberflächenstrukturell orientiert. Er weist allerdings - differenzierend im Vergleich mit gängigen Darstellungen - darauf hin, daß in Sätzen mit Temporaladverbial "für das Verständnis der Aspektkategorie" es wichtig ist, "deutlich zwischen zeitstellenmäßiger Kennzeichnung, die durch zeit-wann-Adverbien erfolgen kann, und aspektueller Kennzeichnung zu unterscheiden" (ibid. 5).

Diese Differenzierung ist vorrangig für die Beziehungen des unvollendeten Verbalaspekt zu Advb Temp relevant. Damit sollen die Unterschiede in den beiden möglichen Lesarten des folgenden Satzes beschrieben und mit den allgemeinen Aspektmerkmalen in Einklang gebracht werden.

"Eine Außerung wie:

(12) VČera v sem' Časov Ivan prinimal lekarstvo. Ivan hat gestern um 7 Uhr seine Medizin genommen.

erlaubt sowohl die aktuelle als auch die nicht-aktuelle Interpretation. In der aktuellen Interpretation fungiert die zeitangabe als aspektueller Referenzpunkt ...

In der nicht-aktuellen Interpretation bezieht sich die zeitbestimung dagegen nicht auf einen, sondern auf mehrere zeitpunkte, die nur eine gemeinsame Bezeichnung haben. Die Außerung referiert auf eine usuelle Handlung, die normalerweise $z u$ dem genannten zeitpunkt vollzogen wird" (ibid. 5 f). 
Aus den Darlegungen Mehligs folgt für die hier gestellte Frage nach den Relationen zwischen PP und verbalaspekt die primär wichtige Feststellung, daß für die Beschreibung der unterschiedlichen Funktionen des unvollendeten Aspekts in Sätzen mit Advb Temp loc zwischen "zeitstellenmäßiger Kennzeichnung" und "aspektueller Kennzeichnung" differenziert werden muß: liegt "zeitstellenmäвige Kennzeichung" vor, ist die Verwendung des. unvollendeten Aspekts durch das Merkmal "Aktualität" gekennzeichnet; liegt "aspektuelle Kennzeichnung" vor, ist der unvollendete Aspekt durch die Merkmale "Aktualität, - Totalität" markiert. Mit der semantischen Unterscheidung der Beziehung des unvollendeten Verbalaspekts zu den "Zeit-wann-Adverbien" deckt Mehlig die asymmetrischen Verhältnisse auf, die hier zwischen der einen syntaktischen Form - Vb Advb Temp ${ }_{\text {LA }}$ - und den mehrdeutigen semantischen Beziehungen ihrer Glieder zueinander vorliegen. Damit führt er zu der Frage, ohne sie jedoch m.w. zu behandeln, die sich aufgrund der breiten Substitutionsmöglichkeit von Prädikaten im unvollendeten und vollendeten Aspekt in Sätzen mit Advb Temp stellt, nämlich: Ist mit dem Hinweis auf die hier vorliegenden asymmetrischen Verhältnisse von syntaktischer Form und ihrer semantischen Mehrdeutigkeit der sprachliche Tatbestand erschöpfend beschrieben, oder lassen sich die semantischen Beziehungen im zusammenhang mit anderen, evtl. komplexeren syntaktischen Formen adäquater einordnen? Bevor ich zur Beantworung dieser Frage komme, sollen noch einige Beispiele aus der Belegsammlung angeführt werden, wobei ich mich auf solche beschränke, in denen das Advb Temp ${ }_{10 c}$ eine relativ kurze zeit bezeichnet:

(70) $\mathrm{V}$ vozduche u šara zaputalas' verevka. No v trudnuju minutu ucenyj ne terjalsja.

Der unvollendete Aspekt ist auch im Falle der affirmativen Form des Prädikats möglich: 
(71) ... V trudnuju minutu učenyj terjalsja, prichodilos' emu pomot'.

(72) Anja pisala éto pis'mo $v$ minutu ot ̌ajanija.

(73) No gordyj charakter ziny ... uvlekal ee $v$ ètu minutu iz sredy vsech priličij.

(74) Varvara Pavlovna ... vo vse vremja uchaživanija molodogo Celoveka $i$ daže $v$ samoe mgnovenie priznanija sochranjala obyčnuju bezmjatežnost' ... duši.

Bei der nun folgenden Behandlung des Problems, ob sich formale Kriterien ermitteln lassen, die über die Beziehungen zwischen Verbalaspekt und Advb Temp etwas aussagen, sollen die Substitutionsmöglichkeiten des Prä̀dikats im unvollendeten und vollendeten Aspekt überprüft werden. Das geschieht mit Hilfe des folgenden Tests:

\section{Ausgangssatz:}

(8) Chimik Mendeleev podnjalsja $v$ tot pamjatnyj den' $v$ vozduch na vozdušnom క̌are.

2. Ausgangssatz ohne Advb Temp loc

(a) Chimik Mendeleev podnjalsja $v$ vozduch na vozdušnom šare.

3. Substituion des Verbalaspekts in (a):

(b) Chimik Mendeleev podnimalsja $v$ vozduch na vozdušnom šare.

4. Erweiterung von (b) mit Advb Temp ${ }_{\text {loc }}$ :

(c) Chimik Mendeleev podnimalsja $v$ tot pamjatnyj den' $v$ vozduch na vozdušnom šare.

Diese Uberprüfungsprozedur ergibt:

1. Bei der überwiegenden Mehrzahl der Fälle erhält man für (a), (b) und (c) richtige Sätze; native-speaker-Informanten bestätigten oder wiesen selbst auf die mit dem Aspektwechsel verbundenen Bedeutungsunterschiede der Aussage hin, die aber 
zu keiner Blockierung der Erweiterung des Satzes mit Advb Temp loc führen.

2. Es läßt sich eine weitere Gruppe unterscheiden, für die die Analyse (a), (b) und (c) auch richtige Sätze ergibt; aber (a) und (b) weisen durch den Aspektwechsel Bedeutungsunterschiede auf, die sich nicht auf die Aspektopposition allein zurückführen lassen. Diese Bedeutungsunterschiede gehen vielmehr auf die Zugehörigkeit der Verben zu bestimmten semantischen Klassen zurück, deren lexikalische Merkmale in der Regel bei der Verwendung der Aspekte - im Verhältnis zu deren Grundbedeutungen- spezifische Bedeutungen implizieren , wie z.B. im unvollendenten Aspekt "Wiederholung der Handlung":

(75) V drugoj den' క̌xukov prišel eše pozdnee.

(a) Šxukov prišel ešce pozdnee.

(b) Ščukov prichodil ešče pozdnee.

(c) V drugoj den' Ščukov prichodil esče pozdnee.

oder "Fehlen des Resultats der Handlung":

(76) Mne podarili èti Casy $v$ den' roždenija.

(a) Mne podarili èti Casy.

(b) Mne darili èti časy.

(c) Mne darili èti casy $v$ den' roždenija.

Die Ergebnisse aus der Analyse dieser Gruppe sind deshalb aufschlubreich, weil sie zeigen, daß bei Bedeutungsunterschieden der Aussage im zusammenhang mit der Substitution des Verbalaspekts dabei nicht das Temporaladverbial als auslösender oder mitspielender Faktor gewertet werden kann, und daB, wie dann $z u$ erwarten ist, in (c) der Bedeutungsunterschied von (b) im Verhältnis $z u$ (a) vorliegt.

3. In einzelnen Fällen ergibt (c) einen nicht korrekten Satz, während (a) und (b) korrekt sind. Man vgl.: 
(77) ... on pomnit, kak $v$ odno utro ona vbezala $v$ kabinet.

(a) $\quad .$. on pomnit, kak ona vbežala $v$ kabinet.

(b) $\ldots$ on pomnit, kak ona vbegala $v$ kabinet.

(c) *... on pomnit, kak $v$ odno utro ona vbegala $v$ kabinet.

Die Faktoren, die zu der Regelverletzung in (c) führen, kommen unten (S. 164) zur Sprache. Zur Illustration des Problems soll noch folgender "ähnlicher" Satz dienen, in dem die Aspektsubstitution möglich ist:

(78) On pomnit, kak $v$ odin prekrasnyj den' ona vbežala $v$ kabinet, vsja krasnaja i rastrepannaja.

(79) On pomnit, kak $v$ odin prekrasnyj den' ona vbegala $v$ kabinet, vsja krasnaja i rastrepannaja.

Die Ergebnisse von 1. - 3. erlauben es, folgende Regel zu

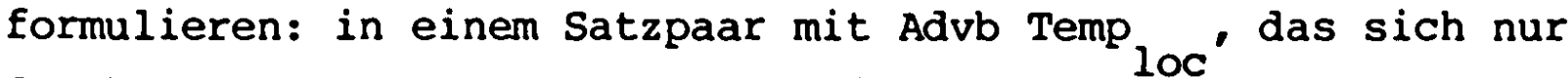
durch den Verbalaspekt unterscheidet, ist der Unterschied eine Variable, die durch den Aspektunterschied bedingt ist.

Es soll nun die Frage, in welchem formalen sprachlichen Zusammenhang die Voraussetzungen für die mehrdeutigen semantischen Beziehungen zwischen unvollendetem Aspekt und Advb Temp ${ }_{\text {loc }}$ zu suchen sein mögen, wieder aufgenommen werden.

In Kap. 4.1.1 wurde die Frage nach den syntaktischen und semantischen Beziehungen der Advb Temp ${ }_{\text {loc }} z u$ den übrigen Satzgliedern behandelt. Als Ergebnis konnte festgestellt werden, daß Advb Temp loc tiefenstrukturell die Funktion einer Prädikation hat: es wird über das finite Verb prädiziert. Schema $3(a / b)$ veranschaulicht diesen Aspekt der Ableitung

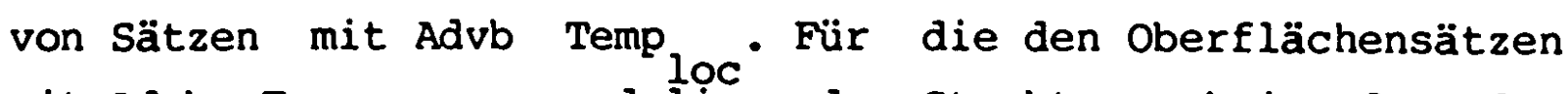
mit Advb Temp ${ }_{\text {loc }}$ zugrundeliegenden Strukturen heist das, daß Advb Temp loc über die nominalisierte form von VP ausgesagt wird (s. $\mathrm{s}_{\mathrm{TS}}^{3}$ der Beispiele). In der nominalisierten Form 
aber ist die Aspektkorrelation neutralisiert. Mit dieser Konzeption der zugrundeliegenden syntaktischen Verhältnisse erfahren die Beobachtungen, daß für die Erweiterungsmöglichkeit von Sätzen mit Advb Temp loc der Verbalaspekt in der Regel nicht relevant ist, ihre systematische Einordnung. In Oberflächensätzen kommen mit der verwendung der finiten verbformen und ihrer Differenzierung in den unvollendeten und vollendeten Aspekt die damit verbundenen Bedeutungsunterschiede zum Tragen und interpretieren die "Zeit", die mit Advb Temp loc genannt ist auf unterschiedliche weise, z.B. wie $0 . z^{10 C^{\prime}}$ " sem' Casov" ausgeführt. Dieser Beschreibungszusammenhang macht auch plausibel, daB die Bedeutung des Temporaladverbials vorrangig intensional ist und das, obwohl unser Bewubtsein von der zeit vornehmlich durch deren extensionale Bedeutung bestimmt ist (Wunderlich (1970), 320). Das heibt, in der Regel kann auch eine "kurze zeit" aufgrund der Interpretation durch den unvollendeten Aspekt die intensionale Bedeutung eines "Ablaufens" dieser zeit bekommen (s. Beipiele 70 - 74). Daß dieser Interpretationsspanne allerdings Grenzen gesetzt sind, zeigt (77): Hier ergibt nur die Kombination der Handlung als telisch im Zusammenhang mit einer bestimmten zeit, die als Moment verstanden wird, einen Sinn. Das bedeutet für die Wahl des Verbalaspekts, daß nur der vollendete Aspekt zulässig ist.

Aus diesen Ergebnissen können auch Schlüsse über die Explikationskraft tiefenstruktureller Analysen in ihrem Verhältnis zu Oberflächensätzen gezogen werden: Mit der Darstellung der zugrundeliegenden Strukturen, im besonderen der Neutralisierung der Aspektkorrelation, wird plausibel, daß für den Oberflächensatz die Möglichkeit der Verwendung beider Verbalaspekte offen ist. Sie liefert aber keine Kriterien für die Wahl des Aspekts im konkreten Fall. Dafür bedarf es der Analyse anderer Strukturen, in denen z.B. die Aspektkategorie verwirklicht ist und die den Oberflächenstrukturen "näher" sind als die hier untersuchten Strukturen. 
Jedoch konnte das Problem "Temporaladverbial und Verbalaspekt" in den tiefenstrukturellen Beschreibungszusammenhang der Adverbialen eingeordnet werden, gemä $B$ dem das Temporaladverbial als prädikat zu der nominalisierten Form des finiten Verbs ( $s^{3}$ Ts ) fungiert.

Betrachtet man die sprachlichen Formen von dieser Beschreibungsebene, lassen sich folgende Feststellungen machen :

1. Für den nominalisierten oberflächensatz von $s^{3}$ TS ist die Aspektkorrelation neutralisiert, damit entfällt eine Abhängigkeit des Verbalaspekts vom Temporaladverbial. In der Bewertung der Relationen zwischen sprachlichen Formen können wir diese Ebene als die allgemeinere bestimmen. Denn ihre Verhältnisse sind

2. im Oberflächensatz mit finitem Prädikat und Temporaladverbial als PP sichtbar, und zwar in der Tatsache, daß wir in diesen Sätzen die weitverbreitete "konkurencija vidov" (Rassudova (1968), 7) vorfinden. 
4.4 ZUM PROBLEM DER MEHRDEUTIGKEIT IN SÄTZEN MIT TEMPORALADVERBIAL

\subsection{MEHRDEUTIGE RELATIONSMÖGLICHKEITEN DER PP}

Mehrdeutigkeit, bedingt durch mehr als eine Relationsmöglichkeit der PP im Satz, ist für die Beschreibung der $P P$ temp weniger relevant als für die $P P$. Die Analyse der bisher diskutierten Beipiele mit Advb Temp ${ }_{\text {loc }}$ entspricht in dieser Hinsicht prädikatenlogischen Darlegungen, nämlich, daß das lokalisierende Temporaladverbial über Argumente der Klassen "Ereignis"/"Umstand" und "Vorgang" ausgesagt wird, das heist für die objektsprachlichen Relationen-über das finite Prädikat bzw. seine Proformen und Nominalisierungen.

Interessant sind in diesem Zusammenhang Sätze, in denen mehrere Prädikate, die als Argumente der oben genannten Klassen fungieren können, vorkommen. Oberflächenstrukturell sind das Sätze mit finitem Prädikat und Infinitiv nach Infinitiv-Tf, Sätze mit Adverbialpartizip, mit Verbalnomina etc. Man vgl.:

(80) Lavreckomu chotelos' v ètot mig ubit' sebja.

(81) No neredko nekotorye prevratnosti boja zastavljjali $v$ te mesjacy projavljat' bespokojstvo i mne.

(82) Puškin ubedil artista $v$ eto vremja prinjat'sja za svoi vospominanija.

(83) My priglasili vas $v$ ijule prinjat' učastie $v$ nasej konferencii.

(84) Ne stanem gadat', kakoj moščnyj stimuljator ... zastavil Adlerskuju notarial'nuju kontoru $v$ mgnovenie oka oformit' pravo na nasledovanie.

(85) On pomogal mne $v$ éti dni pereechat' na novuju kvartiru.

(86) Vladimiru Il'iču bylo razrešeno terez god vernut'sja v Kazan'. 
Die Sätze haben zwei Lesarten: das Temporaladverbial kann nähere Bestimmung zum finiten Prädikat oder zu dem von diesem abhängigen Infinitiv sein. Der Prädikations-Test veranschaulicht die unterschiedlichen tiefenstrukturellen Prädikationsmöglichkeiten:

$\begin{aligned} \mathrm{s}^{3 a} & =\left\{\begin{array}{l}\text { Želanie Lavreckogo } \\ \text { To obstojatel'stvo, cto L. chotel }\end{array}\right\} \text { ubit' sebja, } \\ & \text { imelo mesto v ètot mig. }\end{aligned}$ $\begin{aligned} S^{3 b} & \text { TS Ubienie samogo sebja dolžno bylo imet' mesto } \\ & v \text { ètot mig. }\end{aligned}$

Nebenbei sei bemerkt, daß die Bedeutung der Mehrdeutigkeit für die Kommunikation von unterschiedlichem Gewicht ist. Das ist jedoch eine Frage der lexikalischen Besetzung der in Relation tretenden Satzglieder und nicht der Grammatik. Man vgl. $z u(80)$ :

(87) Lavreckomu chotelos' v mae ubit' sebja.

Diese mehrdeutige Prädikationsmöglichkeit der Advb Temp ist allgemein in Sätzen mit mehr als einem Prädikat $\mathbf{l o c}$ beobachten, dabei können diese weiteren prädikativen Ausdrücke in verschiedenen oberflächenstrukturen erscheinen, z.B.:

- als Adverbialpartizip:

(88) Ja zastal ego v 10 Kasov besprobudno spavక̌im $v$ svoej posteli. (Nach Rủžička (1980), 121).

(89) Ona oșiblasas' v molodosti stav artistkoj. (Nach Rüžička (1980), 176).

(90) Priklkadyvajass' ko krestu po okončanii vsenočnoj ona takže pocelovala ... ruku svjaక̌̌ennika. 
- als Adjektiv (wir übernehmen das Beispiel von Iordanskaja (1967), zit. nach Kibrik (1977), 172), auch wenn das Advb Temp loc hier heute lexikalisiert ist und die Form eines Adverbs hat):

(91) V ètoj gimnazii uč́ilsjja vposledstvii vsemirno izvestnyyj kinoartist.

- nach Nominalisierungs-Tf:

(92) Sobljudenie pravil v igre vyrabatyvaet sderžannost' .

- nach Hebungs-Tf (s.o. (40) S. 149) unterschiedlicher Ableitungsprozeduren:

(93) Vor uvidel milicionera pri pobege.

(93a) Komendant uvidel zaključennogo pri pobege.

Es wurden oben ( $S .144 \mathrm{ff}$ ) Sätze mit Temporaladverbialen untersucht, in denen die NP der Advb Temp loc mit einem NP $_{\text {verb }}$ besetzt ist; dabei konnten strukturelle Besonderheiten festgestellt werden, $z \mathrm{u}$ denen Tilgung der Subjekt-NP und Hebungs-Tf gehören. In (93a) schafft die lexikalische Besetzung der Satzglieder die Voraussetzungen für mehrdeutige Beziehungen der syntaktisch homonymen Funktionen der NP und dem Advb temp So kann "pri pobege" sich einerseits auf NP (93), andererseits auf NP (93a) beziehen.

Uber formale Möglichkeiten und Grenzen der Disambiguierung dieser Art Sätze s. unten.

Den Verhältnissen von (93) ist vergleichbar:

(94) My uznavali opriezde druga na prošloj nedele.

Fragen wir nach Faktoren, die diese Mehrdeutigkeit disambiguieren, so stoßen wir für die geschriebene Sprache - analog zu Sätzen mit lokalen Adverbialen - auf die Wortfolge. 
In den Sätzen (80) - (94) stehen die Advb Temp loc in der Regel zwischen finitem prädikat und dem weiteren prädikativen Ausdruck. Ich verweise auf die Ausführungen über Wortfolgeregeln für Adverbiale im Rahmen der aktuellen Satzperspektive (s.o. Kap. $3.2 \mathrm{im}$ bes. Kap. 3.2.2, S. $106 \mathrm{ff}$ ). Danach kann die Position der PP temp in (80) - (94) die der Grenzstellung zwischen Thema und Rhema sein. Daraus folgt die potentielle Möglichkeit der Prädikation der Adverbiale über das finite Prädikat oder (vel et aut) über den weiteren prädikativen Ausdruck. Ein Positionswechsel der PP femp führt in der Regel zur Disambiguierung ihrer Prädikation.

Die Regeln, die sich aus der T-C-A für die Möglichkeiten der Prädikation der PP ergeben, sind weniger restriktiv, als das bei Lokaladverbialen der Fall ist, was als eine Folge der im Verhältnis $z u$ den PP loc ganz allgemein beschränkten Prädikationsmöglichkeiten der PP $z$ u sehen ist.

Folgende Beispiele sollen die disambiguierende Bedeutung der

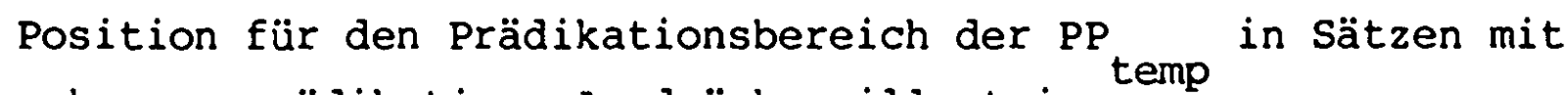
mehreren prädikativen Ausdrücken illustrieren:

(95) V ètot mig Lavreckomu chotelolos' ubit' sebja.

(96) Lavreckomu chotelos' ubit' sebja v ètot mig.

(97) V nespokojnoe vremja tjažlolo okazat'sja snjatym s divizii (Nach Rúžiłka (1980), 135).

(98) Tjaželo okazat'sja snjatym_s divizii v nespokojnoe vremja.

(99) V igre sobljudenie pravil vyrabatyvaet sderZannost'.

(100) Sobljudenie pravil vyrabatyvaet v igre sderžannost'.

(101) Sobljudenie pravil vyrabatyvaet sderŽannost' v igre.

(102) Vor uvidel pri pobege milicionera.

(102a) Komendant uvidel pri pobege zakl juČennogo.

(103) My uzznavali na proకloj nedele o priezde druga.

Die unterschiedlichen Prädikationsbeziehungen der Advb Temp ${ }_{10 c}$ in entsprechenden Positionen bei den Beispielen (93) 
- (94) und (102) - (103) einerseits und den übrigen andererseits (wobei (99) - (101) Illustrationen zu beiden Subklassen sein können), erinnern an die oben - Sätze mit PP nach dem finiten Verb (S. $114 \mathrm{f})$ - behandelten Probleme: in Sätzen mit durch objekte erweiterten VP bedeutet. der Stellungswechsel der PP von der letzten Position in die vorletzte eine Disambiguierung der $T-C-A$ und der Prädikationsmöglichkeiten der PP. Daß Sätze mit Hebungs-Tf wie (93) - (94) bei der Erweiterung durch freie Adverbiale hier dieselben Regelmäßigkeiten aufweisen wie Sätze, in denen die Verbergänzungen keine weiteren abgeleiteten Funktionen aufweisen, bestätigt die wiederholt gemachte Beobachtung, daß die Erweiterung von Sätzen mit freien Adverbialen auf einer relativ "hohen" Stufe ihrer Derivation anzusetzen ist.

Sätze, die nach einer komplizierten Derivationsgeschichte mehrere prädikative infinite Formen auf der Satzoberfläche generieren, können aber auch Beispiele für Blockierungen der Erweiterungsmöglichkeit mit PP ${ }_{\text {temp }}$ sein. Das soll an einem Satz illustriert werden, der - verkürzt - Růžið̌ka (1980) 172) entnommen ist.

Der Satz lautet:

"Gljadja na bojcov $i$ oficerov, ničego ne podozrevaja begavšich u ešelonov, ja to $i$ delo vspominal, čto ..." Die Erweiterungsmöglichkeit durch eine PP in der Position zwischen Adverbialpartizip und Partizip temp blockiert. Man vgl.:

(104) *Gljadja na bojcov $i$ oficerov, ničego ne podozrevaja v èto vremja begavšich u ešelonov, ja to i delo
vspominal, cto $\ldots==0$

Dagegen erhalten wir korrekte Sätze bei Positionswechsel der $\mathrm{PP}$ oder Verwendung einer anderen Oberflächenform als der infiniten: 
(105) Gljadja na bojcov i oficerov, niと̌ego v èto vremja ne podozrevaja begavšich u eselonov, ja to i delo vspominal, tto ...

(106) Gljadja na bojcov i oficerov, ni ̌ego ne podozrevaja begavšich $v$ èto vremja u ešelonov, ja to $i$ delo vspominal, tto ...

(107) Gljadja na bojcov i oficerov, kotorye ničego ne podozrevaja v èto vremja begali $u$ eselonov, ja to $i$ delo vspominal, tto ...

Die Untersuchungen von Advb Temp ${ }_{\text {loc }}$ in Sätzen mit mehreren prädikativen Ausdrücken erlauben, folgende Regeln zu formulieren:

1. Die Prädikationsmöglichkeiten der PP temp ergeben sich in Abhängigkeit von der Anzahl der prädikativen Ausdrücke im Satz.

2. Die Position der PP temp ist ein disambiguierenden Faktor, sofern nicht der Fall eintritt, daß sie an der möglichen Grenze von Thema und Rhema steht.

3. Sätze mit mehreren prädikativen Ausdrücken und der PP in der Position zwischen diesen sind oberflächenstrukturell mehrdeutig. (Für Sätze mit Hebungs-Tf und Erweiterung durch PP temp gelten die Regeln von Kap. 3.2.2.2, s. bes. S. $115 \mathrm{f}$ ). 


\subsubsection{MEHRDEUTIGKEIT DER KLASSE DER ADVERBIALE}

Bei der Beschreibung von Adverbialen klassifiziere ich diese unter Zuhilfenahme auch prädikatenlogischer Fragestellungen. Die Mehrdeutigkeit von Sätzen mit PP, die im folgenden zur Sprache kommen soll, beschränkt sich auf solche Fälle, bei denen die Frage nach der Klasse der Prädikation nicht eindeutig $\mathrm{zu}$ beantworten ist.

In linguistischen Arbeiten sind weitgehend brauchbare Beschreibungen für die Unterscheidung von lokalen und temporalen Adverbialen entwickelt worden (Steinitz (1971), 72 f), be $\frac{i}{2}$ denen bekanntlich eine Reihe von präpositionen analog verwendet wird.

Jedoch erfassen die Regeln über die Merkmalmatrix der NP in der PP: (- abstrakt) für Lokaladverbiale und (+ abstrakt) für Temporaladverbiale z.B. folgende Fälle nicht:

(108) Oni dolgo bluždali v tumane.

(109) Na prošedక̌em pisatel'skom s''ede naibolee často nazyvalis' imena takich gorjačich priveržencev sel'skoj temy.

(110) V prezidiume ... soprovoždajuščie ego $\mathrm{v}$ poezdke indijskie gosudarstvennye dejateli.

(111) ... Cto ja takie minuty ... ščitaju tak redko, とto ne mogu ne povtorjat' ètich minut $v$ mečtan'jach.

Die PP kann in diesen Sätzen sowohl als nähere lokale wie auch als nähere temporale Bestimmung des finiten Prädikats verstanden werden.

Betrachten wir die tiefenstrukturellen Verhältnisse gemäß dem hier erstellten Modell, so ergeben sich als stufen der Ableitung: 
$\mathrm{S}_{\mathrm{TS}}^{1}$ : Oni dolgo bluždali.

$\mathrm{s}_{\mathrm{TS}}^{2}$ : Byl tuman.

Eine Prädikation von $s^{2}$ TS über $s^{1}$ TS generiert Sätze mit unterschiedlichen Klassen des Prädikat-Argument-Verhältnisses; z.B. mit der einer kausalen Relation.

(112a) Bluždenie ich imelo mesto iz-za tumana.

Das formale Kriterium, die Relation als kausale $z u$ unterscheiden und $z u$ erkennen, liefert die Präposition "izza", deren Verwendung bekanntlich nur mit eindeutiger Bedeutung, nämlich Angabe der Ursache, erfolgt.

Bei dem versuch, $s^{2}$ in eine lokale Relation zu $s^{1}$ is setzen, stoßen wir auf die Tatsache, daß die angeführten formalen Kriterien, Präposition oder Merkmalmatrix der NP der PP, nicht ausreichen, um für (108) eine Unterscheidung von lokaler und temporaler prädikation auszudrücken:

(112a) Bluždenie ich imelo mesto $v$ tumane.

Wir müssen zu expliziteren Oberflächenstrukturen greifen, z.B.

(113) Oni bluždali tam, gde byl tuman.

(114) Oni bluždali, kogda byl tuman.

Dennoch kann man sagen, daß in der Regel lokale und temporale Adverbiale aufgrund der distinktiven Merkmalmatrizes der NP der PP voneinander abgegrenzt werden können. Unsere Beispiele (108) - (11l) weisen auf solche Fälle hin, in denen in dem präpositionalen Ausdruck solche Nomina Verwendung finden, für die die üblicherweise zugrundegelegte Merkmalmatrix nicht präzise genug ist, damit fehlt das Unterscheidungskriterium. Diesem metasprachlichen Sachverhalt entspricht objektsprachlich, daß die PP zweideutig ist. Das Unterscheidungskriterium für lokale und temporale prädikation stützt sich bei gleicher 
Präposition auf die binäre Opposition von ( \pm abstrakt) des Nomens der PP. Für die Mehrzahl der Adverbialklassen ist dieses Unterscheidungskriterium jedoch irrelevant, da es sich bei der Prädikation durch Adverbiale voiwiegend um abstrakte Relationen handelt wie modal, kausal, konzessiv.

Zwar beschränkt sich diese Arbeit auf die "elementaren" Adverbiale - lokale, temporale, modale -; dennoch sollen hier einige Beobachtungen $\mathrm{zu}$ dem Problem der Mehrdeutigkeit auch im Hinblick auf andere Subklassen zur Sprache kommen. Gerade die Temporaladverbiale liefern Argumente, die sprachlichen Zusammenhänge dabei darzustellen, da bei der Analyse ihrer verwendung deutlich wird, das - neben der doch als peripher $z u$ bezeichnenden Mehrdeutigkeit lokal/temporal - im besonderen PP mit den Präpositionen "pri" und "po" in bezug auf die Adverbialklassen temporal, modal, kausal, konditional sowie auf Satzgefüge mit "und" mehrdeutig sind.

Es werden zunächst Belege mit der Präposition "pri" diskutiert: In der Literatur (z.B. Wunderlich (1970), 250) ist die semantisch nahe Beziehung zwischen Konditional- und Temporalsätzen untersucht und beschrieben worden. In diesem Zusammenhang ist die Mehrdeutigkeit der Präposition "pri" zu sehen: die PP bestehend aus "pri NP "kerb" können in der Regel sowohl temporal als konditional verstanden werden, disambiguierende Faktoren sind neben dem Kontext Tempus und Modus des Prädikats im Hauptsatz.

Darüberhinaus gibt es solche Belege mit (pri NP verb), in denen die PP zum Ausdruck einer satzsemantischen $\stackrel{\text { verb }}{\text { Relation }}$ verwendet wird, die weder als temporal noch als konditional verstanden werden kann. Man vgl.:

(115) (Po vyplavke Cuguna i stali SSSR davno vyకel na pervoe mesto $v$ mire.) I vse ze pri burnom razvitii masinostrojenija ... my oscuscaem deficit metalla. 
Die Lesart der PP in (115) ist nicht temporal, nicht konditional (und auch nicht konzessiv), obschon es naheliegend ist, auf einen "ähnlichen" Satz zu verweisen, in dem die gleiche PP - in Abhängigkeit von den genannten Faktoren temporal oder konditional verstanden wird. Man vgl.:

(116) Okružajušaja sreda $\left\{\begin{array}{l}\text { zagrjaznjaetsja } \\ \text { zagrjaznajalas }\end{array}\right\}$ pri burnom razvitii mašinostroenija.

Entsprechende Beobachtungen lassen sich z.B. an folgenden Sätzen machen:

(117) ... posredstvom proletarskogo apparata, pri rukovodstve partii, osušestvljaetsja diktatura klassa.

(118) Planovye pokazateli ne vypolnjajutsja pri nekvalificirovannom rukovodstve.

(119) Rabotu ètu načal ... N.P. Il'in; zaverłil ee A.E. Parnis pri uxastii drugich issledovatelej.

Bei Verwendung ${ }^{43}$ einer konjunktivischen Verbalform des Prädikats kann die PP von (119) auch eine konditionale Relation zum Prädikat ausdrücken:

(121) Mne chotelos' by pri uxastii lučsich sovremennych pisatelej sozdat' celuju seriju knig dlja detej.

Um welche satzsemantische Bedeutung neben temporal und konditional handelt es sich bei der verwendung der PP (pri NP verb' in den "ähnlichen" Satzpaaren (115) - (119), (121), und wie läßt sich diese Mehrdeutigkeit mit Hilfe der tiefenstrukturellen Analyse beschreiben?

Der Prädikations-Test, angewendet auf (115) und (116) ergibt zunächst für (115): 
$\mathrm{S}_{\mathrm{TS}}^{1}$ : Ošcušaetsja deficit metalla.

$\mathrm{S}_{\mathrm{TS}}^{2}$ : Burno razvivaetsja mašinostroenie.

Für $(116)$ :

Sl TS : Zagrjaznjaetsja okružajuščaja sreda.

$\mathrm{S}^{2} \mathrm{TS}$ : Razvivaetsja małinostroenie.

Setzen wir $S_{T S}^{1}$ und $S_{T S}^{2}$ zueinander in Beziehung, und zwar in der Form, das $S^{2}$ TS über $S^{1}$ TS ausgesagt wird, und bedienen wir uns dabei der PP in der Besetzung (pri NP Fall ein, daß nur die Präposition als Unterscheidungskriterium $z u$ anderen möglichen Relationen fungiert (s. dazu o.S. $146 \mathrm{f})$. Die bekannte Mehrdeutigkeit von "pri" hat die Mehrdeutigkeit von (115) - (119), (121) zur Folge, was nicht der Fall wäre bei Ausdruck einer kausalen Relation durch z.B. die PP (iz-za NP verb):

(122) Oscušaetsja deficit metalla iz-za burnogo razvitija masinostroenija.

Mit Hilfe des hier verwendeten Beschreibungsmodells lassen sich die formalen Faktoren nennen, die zu der Mehrdeutigkeit in Sätzen mit (pri NP verb) führen. Eine Auflösung der semantischen Mehrdeutigkeit leistet es nicht. Es kann jedoch festgestellt werden, daB - ohne damit in widerspruch $z u$ der tiefenstrukturellen Analyse zu geraten - die Sätze (115) (119), (121) anderen Oberflächenstrukturen nahestehen, die sich auch in der Hinsicht unterscheiden wie die Sätze (115), (117), (119) einerseits und (116), (118), (121) andererseits: ich meine zum einen den temporalen und/oder konditionalen NS (123), zum anderen die PP (s NP verb) (125).

Man vgl.: 
(123) Zagrjaznjaetsja okruŽajuక̌̌aja sreda, $\left\{\begin{array}{l}\text { esli } \\ \text { kogda }\end{array}\right\}$ razvivaetsja mašinostroenie.

Entsprechendes gilt für (118), (121), jedoch nicht für (115), (117), (119), genauer dann nicht, wenn eine Verwendung wie in dem gegebenen Kontext vorliegt. So ist für (115) nicht adäquat:

(124) *Po vyplavke cuguna i stali SSSR davno vyšel na pervoe mesto $v$ mire. I vse $z e,\left\{\begin{array}{l}\text { esli } \\ \text { kogda }\end{array}\right\}$ burno razvivaetsja mašinostroenie, oščušcaetsja deficit metalla.

Für eine positive Bestimmung der hier durch die PP (pri NP yerb' ausgedrückten satzsemantischen Relation liefert uns (119) Argumente. Man vgl. dazu:

(125) Rabotu zaveršil A.E. Parnis s uxastiem drugich issledovatelej.

Die satzsemantische Relation der PP, d.h. tiefenstrukturell von $S^{2} \mathrm{TS}^{\prime}$ zu dem übrigen Satz ist damit die einer Konjunktion (s. Weiss (1974), 201), die sprachlich mit "und", "und gleichzeitig" ausgedrückt werden kann. Diese Verwendung liegt auch bei (115) und (117) vor; man vgl.:

(126) ... I vse že oscušcaetsja deficit metalla $\underline{v}$ naše vremja, $\left\{\begin{array}{l}\text { kogda burno razvivaetsj masinostroenije. } \\ \text { burnogo razvitija maSinostroenija. }\end{array}\right\}$

Diese Zusammenhänge machen deutlich, weshalb das Verständnis der PP als temporal oder konditional für (115), (117), (119) nicht paßt, "zu eng" ist; die Frage nach der Bestimmung der

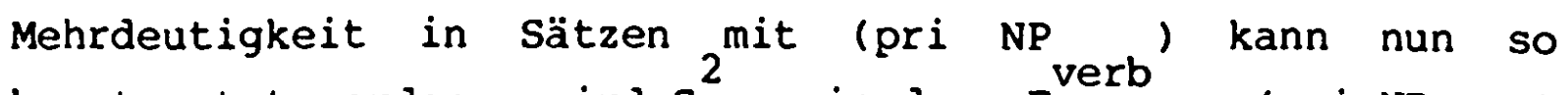
beantwortet werden: wird $S^{2}$ is in der Form von (pri NP verb) 
über $S^{l}$ TS prädiziert, so kann diese Aussage als zeitliche Festlegung oder überhaupt als Bedingung von $S$ verstanden werden (s. (116), (118), (121)). Diese Aussage kann jedoch auch eine "weitere" Bedeutung haben, nämlich die Verbindung zweier Sätze durch das nicht weiter qualifizierende "und", s. (115), (117), (119).44

Die PP (po NP verb) kann eine temporale Relation ausdrücken, nämlich das Nacheinander zweier Ereignisse/Umstände und Vorgänge, z.B. "po priezde", "po ot''ezde", "po vozvrašcenii", "po smerti". Man wird bei einem Vergleich dieser Ausdrücke mit den nun folgenden mehrdeutigen Sätzen mit PP (po NP verb) feststellen, daß das Unterscheidungskriterium, mit dessen Hilfe die obigen Ausdrücke als temporal identifiziert werden, die semantische Merkmalmatrix der NP der PP liefert, die nämlich das Merkmal [Zeitbezug] enthält.

Folgende Sätze mit PP (po NP verb) sind Beispiele für Mehrdeutigkeit, die zu beobachten ist, wenn das Merkmal [Zeitbezug] der $N N_{\text {verb }}$ im Regelfall nicht relevant ist oder entfällt:

(129) Po chodu sceny boja na zastave No. 6 Višnevskij vdrug vychvatil iz kobury revol'ver.

(130) (VozduSnyj korabl' nachodilsja na lune.) Po komande s zemli byla vkljutena bortovaja apparatura.

(131) Priłla vojna. PoSel voevat'. Po raneniju vernulsja domoj ran'še drugich mužikov.

(132) Po ego prikazaniju ja pokinul komnatu. (Jakobson (1935), zit. nach Jakobson (1974), 108)

(133) Po doneseniju gorodni Xego, za nechożenie so krestom o Pasche $v$ doma raskol'nikov, byl snova vyzvan $v$ guberniju.

Uberprüft man die Relation der PP zu den übrigen Satzgliedern mit Hilfe des Prädikations-Tests, so erhält man Ergebnisse, 
die denen mit der PP (pri NP verb) analog sind; man vgl. zu (129):

s'TS : Višnevskij vdrug vychvatil iz kobury revol'ver.

$\mathrm{S}_{\mathrm{TS}}^{2}$ : Sia scena boja (na zastave No. 6 ).

zu $(130)$ :

$\mathrm{s}_{\mathrm{TS}}^{1}$ : Byla vkljußena bortovaja apparatura.

$\mathrm{S}_{\mathrm{TS}}^{2}$ : Komandovali s zemli.

zu $(131)$ :

$s_{\mathrm{TS}}^{1}$ : On vernulsja domoj ran'še drugich muzikov.

$\mathrm{s}_{\mathrm{TS}}^{2}$ : on byl ranen. usw.

Für die Ableitung von (129) - (133) läßt sich mit dem entwickelten Modell wiederum nur beschreiben, daß $\mathrm{s}^{2}$ in der

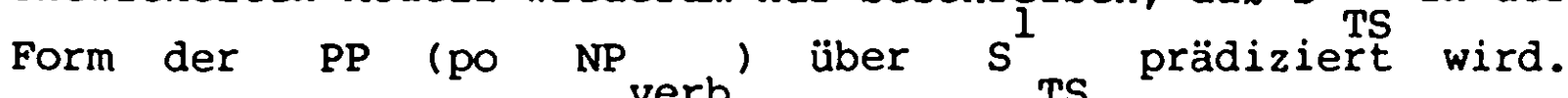

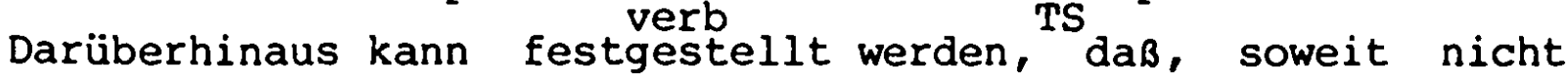
Kontext und/oder "allgemein" semantische Faktoren die so hergestellte Relation qualifizieren, die Präposition der PP das einzige Mittel zum Ausdruck semantischer Unterschiede ist. Aufgrund der Mehrdeutigkeit von "po" können somit sätze mit der PP (po VP verb) mehrdeutig werden. Die Auflösung der Mehrdeutigkeit kann durch Rückgriff auf andere "nahe" Oberflächenstrukturen erfolgen.

Zu (129) kann man hier feststellen, daß es sich bei der PP um einen temporalen Ausdruck handelt, obwohl "po chodu" in der festen wortfügung "po chodu dela" synonym zu "po obstojatel'stvam", d.h. modal verwendet wird.

Nach Auskunft von native-speaker-Informanten kann in (130) "po komande" als Ausdruck einer kausalen oder temporalen näheren Bestimmung des Prädikats verstanden werden:

"potomu cto komandovali" oder "posle togo kak komandovali". 
Die Herausgeber der Erzählungen von Sukšin ${ }^{45}$ interpretieren "po raneniju" in (131) als Ursache: "na osnovanii ranenija"; die PP könnte jedoch auch in der Funktion eines Temporaladverbials verstanden werden: "posle ranenija".

In (132) ist die Wahl zwischen der Bestimmung des Adverbials als modal: "tak, kak on prikazal" -

oder kausal: "potomu cto on èto prikazal" zu treffen.

Entsprechendes gilt für (133):

modal: "tak kak bylo doneseno gorodnicim" -

oder kausal in der Form des nachgestellten Kausalsatzes: "... potomu to bylo doneseno gorodnitim".

Bei der Darstellung der Advb Temp mußte wiederholt das Problem der systembedingten Mehrdeutigkeit behandelt werden (S. $144 \mathrm{ff}, \mathrm{S} .166 \mathrm{ff}, \mathrm{S} .172 \mathrm{ff}$ ). Ziehen wir in diesem Zusammenhang in Betracht, daß auch mit der verwendung der lokalen Adverbiale das Problem der Mehrdeutigkeit verbunden ist ( $S .89 \mathrm{ff}, \mathrm{S} .106 \mathrm{ff}$ ), so wird verständlich, weshalb in nicht transformationellen Untersuchungen die freien Adverbialen, die unter "primykanie", also "Adjunktion" abgehandelt werden, die fast stereotype Beurteilung erhalten: "bestimmen den Satz im ganzen". (Vgl. z.B. "Takoj samostojatel'nyj rasprostranitel', otnosjašijsja ko usemu predloženiju $v$ celom, nazovem det e $\mathrm{m}$ i $\mathrm{n}$ a $\mathrm{n}$ o $\mathrm{m}$ ", Grammatika (1970), \$ 1422; oder "... pozvoljaet vskryt' ich $1=$ determinantov, H.S.) osobuju prirodu kak rasprostranitelej predloženija v celom", Krylova, O. (1976), s. 44.)

Mit Hilfe der generativ-transformationellen Methode wurde die Derivationsgeschichte der Sätze mit Advb Temp loc im Hinblick auf die Rolle des Adverbials im Satz untersucht und 1. dessen vorrangige Beziehung zum finiten Verb festgestellt wie auch 2. dessen semantischer Charakter bestimmt, nämlich als einer Prädikation temporaler Bedeutung. Diese Ergebnisse werden durch prädikatenlogische Analysen gestützt. 
Was bedeutet aber für die syntaktischen Verhältnisse, für die Konstitution des Satzes die Relation des Advb Temp ${ }_{\text {loc }}$ um finiten Verb?

Für die Beantwortung dieser Frage erwiesen sich besonders die Beispiele mit potentieller Mehrdeutigkeit als aufschlußreich (s. besonders Beispiele (31) - (40), S. $147 \mathrm{f}$ und 4.4.2, s. $172 \mathrm{ff}$ ). Es handelt sich dabei um strukturen, in denen einerseits - tiefenstrukturell gesprochen - beide Prädikationen mit ihren Argumenten im Oberflächensatz in Form von NP nom' NP gen oder Possessivpronomina vertreten sein können, ${ }^{n o m}$ sie können aber auch - durch unterschiedliche Bedingungen - getilgt sein. Damit stellt sich für ein generativ-transformationelles Sprachbeschreibungsmodell das Problem, wie - im Hinblick auf einen kontextfreien Oberflächensatz - der Forderung nach "recoverability" (Chomsky (1970), z.b. $222 \mathrm{f}, 228 \mathrm{f}$ ) entsprochen werden soll. Es gibt offensichtlich keinen Mechanismus für die zurückgewinnbarkeit von $\mathrm{NP}^{1}$, dominiert von $\mathrm{s}^{2} \mathrm{TS}$ i die getilgten Satzglieder müssen durch Rückgriff auf Kontext oder "Vorwissen" erschlossen werden. Auf dieses Problem ist in der einschlägigen Literatur hingewiesen worden:

Wunderlich $(1970,222$ f) behandelt Sätze vom Typ:

"(237a) Abel wurde von Kain nach kurzem zögern erschlagen."

- die er für mehrdeutig hält.

Darüber läßt sich wohl streiten. Folgender Satz, in dem die NP nicht durch ein traditionelles Vorwissen bereits interpretiert werden, mag geeigneter sein, das grammatische Problem, das diesen Sätzen gemeinsam ist, zu verdeutlichen, nämlich, daß aus der Derivationsgeschichte keine eindeutigen Regeln für die Besetzung der NP , dominiert von $S^{2}{ } S^{\prime}$ abgeleitet werden können. Man vgl.:

(134) Es hieß, der Angeklagte habe den Dieb nach kurzem widerstand erschlagen. 
Auf Russisch:

(135) Govorili, tto podsudimyj ubil posle nekotorogo soprotivlenija vora.

Befragungen zum deutschen wie zum russischen Satz ergaben, daß hier, im Gegensatz zum verständnis des Satzes aus wunderlich, das Adverbial spontan als nähere Bestimmung zum objekt verstanden wird.

Die Relation des Advb Temp ${ }_{\text {loc }}$ zum finiten Prädikat - in Oberflächensätzen auch $z u$ dessen nominalisierter Form (s. S. 168 (92)) - impliziert die potentielle "Nichtrekonstruierbarkeit" der $\mathrm{NP}^{1}$ von $\mathrm{s}_{\mathrm{TS}}^{2}$, was potentielle Mehrdeutigkeit zur Folge hat.

Zu diesen reduzierten formalen Unterscheidungskriterien der Struktur kommt andererseits noch hinzu, daß die präposition, die bei Advb Temp loc mit NP verb das Element ist, das die klasse der Prädikation ausdrückt, mehrdeutig sein kann - für das Russische liefern die Beispiele (115 ff) Belege dafür. $\mathrm{DaB}$ es sich auch hierbei um übereinzelsprachliche Merkmale handelt, zeigt das 2 itat aus Bartsch (1972) ${ }^{46}$. 


\section{MODALE ADVERBIALE}

In dem allgemeinen Teil (s.o. S. $51 \mathrm{ff}$ ) über die in dieser Arbeit verwendeten Tests zur Ermittlung der die Klasse der fakultativen Adverbiale charakterisierenden Formen und Funktionen wurde darauf hingewiesen, daß diese Tests für die Beschreibung der drei Primärklassen fakultativer Adverbiale von unterschiedlicher Relevanz sind: mit Hilfe von Prädikations-Test und Dialog-Test lassen sich die gemeinsamen formalen und funktionalen Unterschiede der fakultativen Adverbialklassen (hier: lokal, temporal, modal) im Unterschied zu der Klasse der obligatorischen Adverbialen - illustriert für die lokalen - beschreiben. Jedoch zur Beantwortung der Frage nach den Erweiterungsbedingungen von Sätzen mit fakultativen Adverbialen reichen diese beiden Testsorten nicht aus: für die Untersuchung der Lokaladverbiale z.B. ist es notwendig, außerdem Tests zur Uberprüfung der Beziehung zwischen Prädikationsbereich des Lokaladverbials und Thema-Rhema-Gliederung heranzuziehen (s.0. Kap. 3.2, S. $86 \mathrm{ff}$ ). Diese Testsorte erwies sich für die Beschreibung der Temporaladverbiale z.B. als in der Regel nicht relevant. Das unterschiedliche verhalten bestimmter Adverbialklassen auf bestimmte Textsorten läßt auf unterschiedliche Formen und Funktionen dieser Adverbiale bei der Erweiterung von Sätzen durch sie schließen. Die Voraussetzung für diese Unterschiede sehe ich in den Spezifika der durch die Adverbiale ausgedrückten Prädikationen ( $s$. dazu o. Lokaladverbiale S. 65 f). Mit der Einbeziehung dieses Erklärungszusammenhangs werden Ergebnisse prädikatenlogischer Untersuchungen in dieser linguistischen Analyse erprobt und es kann festgestellt werden, daß diese Ergebnisse durch bestimmte sprachliche Regelmäßigkeiten bestätigt werden.

So fallen auch bei der Untersuchung der Modaladverbiale formale Besonderheiten auf, die die Sätze mit fakultativen 
Modaladverbialen ${ }^{1}$ von Sätzen mit Lokal- und Temporaladverbialen unterscheiden. In generativen und prädikatenlogischen Arbeiten über Modaladverbiale, dargestellt am Beispiel des Englischen und Deutschen ${ }^{2}$, sind die satzsemantischen zusammenhänge für die Verwendung von Modaladverbialen untersucht worden. Die Beschreibung der diese Sätze charakterisierenden Formen und Funktionen erfolgt mit Hilfe zweier Testsorten, die im folgenden auf das Russische angewendet werden sollen; es sind dies der Negations-Test und der PrädikatsvariablenTest.

Einleitend soll ein Uberblick darüber gegeben werden, in welchen typischen Oberflächenrealisierungen der russischen Literatursprache Modaladverbiale in Form von PP (im folgenden PP ') vorkommen. Man vgl. die Sätze:

(1) ${ }^{\text {mod } . . . ~ n a ~ k o t o r u j u ~ v y ~ s m o t r e l i ~}{ }^{4}$ inogda s sožaleniem, inogda $s$ kakoju-to sostradatel'noju ljubov' ju.

(2) ... ona tol'ko posmotrela na menja v udivlenii i pošla proc'.

(3) ... perebil ego Panšin s prezritel'noj nebrežnost'ju.

(4) Plachi stojali rjadami, točno rjady pušek s otkrytymi Zerlami, v bezmolvnom ožidanii.

In meiner Beispielsammlung sind unter den $\mathrm{PP}_{\text {mod }}$ am häufigsten die Beispiele mit (s NP instr). Weniger zahlreich sind die

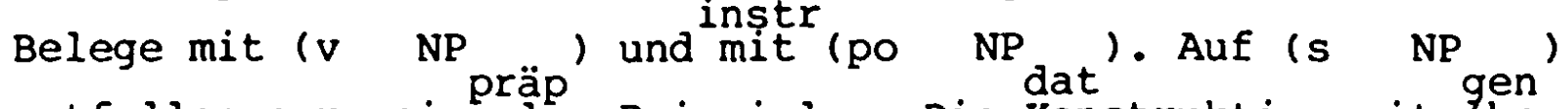
entfallen nur einzelne Beispiele. Die Konstruktion mit len NP gen kann antomym zu ( $s \quad N_{\text {instr }}$ ) und ( $v$ NP Wir wollen die Klassifizierung russischer PP mod mitäfolgenden zwei Fragen beginnen:

1. Ist den Sätzen (z.B. 1-4), in denen die beiden Präpositionalkonstruktionen (s NP instr) und ( $\mathrm{N}$ NP präp) vertreten sind, tiefenstrukturell eine identische $\stackrel{\text { präp }}{\text { instr }}$ tungsstruktur zuzuschreiben oder nicht?

2. Zeichnen sich im Vergleich mit anderen Adverbialen die Modaladverbialen in ihrer Relation $z u$ den übrigen Satzgliedern aus? 
5.1 DER NEGATIONS-TEST BEI MODALADVERBIALEN

Wir nehmen zunächst Frage 2 (S. 184) auf. Diese Frage ist wie angedeutet - in der Forschung von unterschiedlichen theoretischen Standpunkten aus behandelt worden. Vom Standpunkt einer linguistischen Beschreibung fällt auf, daß sich die modalen Adverbiale bei Verneinung ${ }^{5}$ der Aussage nicht wie die fakultativen Lokal- und Temporaladverbiale ${ }_{6}$ sondern wie z.B. die obligatorischen lokalen Adverbiale verhalten, obwohl sie sich ansonsten von diesen in ihren strukturellen Eigenschaften unterscheiden. Man vgl.:

(5) Caušskie promysla raskinulis' po tečeniju Čausa (verst na desjat').

(6) Vsju Žizn' on služil po predpisaniju.

Das Adverbial in (6) ist fakultativ, nicht aber in (5).

(7) *Caušskie promysla raskinulis'.

(8) Vsju žizn' on služil.

Entsprechend ist in (6) die PP auf einen Satz zurückzufüren, nicht aber in (5). Der Prädikations-Test zeigt, daß die PP das Prädikat des Matrixsatzes näher bestimmt. Man vgl.: $\bmod$ (9) Vsju Žizn' on služil tak, kak (emu) predpisyvali. ${ }^{7}$

Bei Verneinung der Aussagen von (5) und (6) scheinen sich die Ergebnisse $z u$ entsprechen; man vgl.:

(10) Čausskie promysla ne raskinulis' po tečeniju Causa. $\longleftrightarrow$

Causskie promysla raskinulis' ne po tečeniju Łauša.

(11) Vsju žizn' on ne služil po predpisaniju. $\longleftrightarrow$ Vsju žizn' on služil ne po predpisaniju. 
Mit Hilfe des Negations-Tests sollen die unterschiedlichen Regelmäßigkeiten bei Verneinung von Sätzen mit Adverbialen festgestellt werden. Die Untersuchung beschränkt sich auf Sätze mit fakultativen Lokal-, Temporal- ${ }^{8}$ und Modaladverbialen.

Der Negations-Test überprüft die Negierbarkeit der Prädikate im Satz. Die bisherigen tiefenstrukturellen Analysen ergaben, daß die fakultativen Lokal- und Temporaladverbiale abgeleitete Sätze sind, womit impliziert ist, daß sie prädikate enthalten. Daraus folgt, $d a \beta$ bei einer Uberprüfung der Negierbarkeit der Prädikate diese Prozedur getrennt auf das Prädikat (Präd) von $S^{1}$ TS und auf das Prädikat (Präd ${ }^{2}$ ) von $\mathrm{s}^{2}$ TS angewendet wird. TS Nebenbei sei erwähnt, daß der Negations-Test damit auch eine den Prädikations-Test ergänzende Prozedur zur Feststellung der syntaktischen Verhältnisse in Sätzen mit Adverbialen ist. Der Negations-Test ergibt in Sätzen mit fakultativem Lokaladverbial und entsprechend mit Temporaladverbial Folgendes:

(12) Olimpijskaja komanda treniruetsja na stadione.

Tf

$\Longrightarrow$ Neg Präd
$\Longrightarrow$ na stadione.

Tf

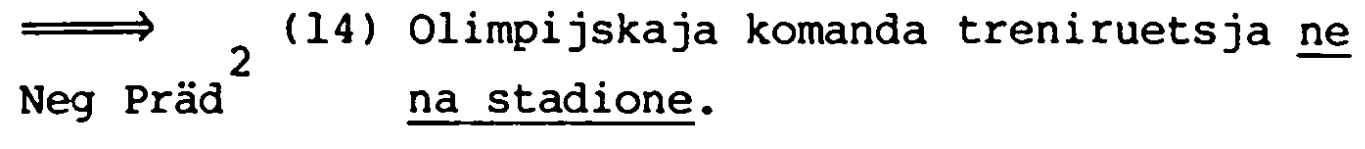

D.h. negierbar ist sowohl die Aussage von Präd ${ }^{l}$ als auch die von Präd. 
Verfahren wir entsprechend bei Sätzen mit Modaladverbialen, so machen wir die Beobachtung, das die Negation, angewendet auf Präd, zwar in der Wortfolge eine Struktur wie (13) ergibt, jedoch ist der wirkungsbereich der Negation ${ }^{9}$ ein anderer, er entspricht (14); man vgl.:

(15) Olimpijskaja komanda treniruetsja s bol'šoj ènergiej.

Tf

$\Longrightarrow$

Neg Präd ${ }^{1}$

(16) Olimpijskaja komanda ne treniruetsja

$s$ bol'šoj ènergiej.

Tf

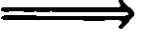

Neg Präd ${ }^{2}$
(17) Olimpijskaja komanda treniruetsja ne s bol'šj ènergiej.

Als Ergebnis der Anwendung des Negations-Tests auf Sätze mit Modaladverbial kann man zunächst die Feststellung machen, daß Präd nicht negiert wird, daß vielmehr bei mehreren wahlmöglichkeiten der Wortstellung von "ne" der Satz bedeutet, daß Präd negiert wird ${ }_{1}$ und zwar in dem Sinn, daß die Modifizierung von Präd ${ }^{1}$ die durch Präd ${ }^{2}$ ausgedrückt wird, verneint wird.

Diese Beobachtungen gelten nicht nur für Sätze mit Modaladverbialen in der Form einer PP, sondern auch in der Form von Adverbialpartizipien. Rủžička (1980, 182 ff) diskutiert in diesem Sinn Sätze wie "(71) on ubil, zašišajas'" und bemerkt dazu: "Wir wollen nun eine Negation zu (71) suchen, ... . Seltsamerweise scheint es keine andere solche Negation zu geben als (74).

(74) Neverno, ťto on ubil, zaščiščjas' " (ibid.). 
Rúzička geht dem logischen Sachverhalt nach, der in Sätzen wie (71) ausgedrUckt wird und sieht dessen Quintessenz darin, daß "die wahrheit von on ubil" ..." in (74) ... keineswegs negiert" ist. "Sie ist vielmehr vorausgesetzt, wenn man will, eine Präsupposition" ... . (71) und (74) würden "sich überhaupt einer wahrheitsbewertung verschlieben, wenn der Sachverhalt, den on ubil beschreibt, nicht als Tatsache vorausgesetzt wird. So ist es also nur eine Negation der e in e n, als Gerundium zašxišajas' kondensierten Proposition, die in der Negation von (71) erzielt wird, wie sie (74) ausdrückt: Es wird behauptet, daB die Handlung, die das Gerundium beschreibt, nicht stattfand. Aber die, Feststellungen über die beiden Propositionen und ihren wahrheitswert im einzelnen müssen mit der semantischen Funktion des Gerundiums verbunden werden. Denn von ihr ist der Wirkungsbereich der Negation abhängig" (ibid. 183).

Rủzicka sieht die Nähe der mit (71) und (74) beschriebenen Strukturen zu Modaladverbialen in der Form einer PP ${ }^{10}$ :

"Wenn wir durchaus mit Verwendung der Negationspartikel ne eine Bedeutung erzielen wollen, die der von (74) nahe- oder gleichkommt, müssen wir zu etwas Ähnlichem wie (83) greifen: (83) on ubil ne $v$ porjadke samozaščty" (ibid. 185).

Jedoch hebt Rủzicka im folgenden auf die Unterschiede dieser Konstruktion $z u$ Sätzen mit Adverbialpartizip ab. Mir scheint dagegen die strukturelle Verwandtschaft von (83) und (74) einen plausibleren Beschreibungszusammenhang für den "Wirkungsbereich der Negation", der in Sätzen vom Typ (74) und unserem Beispiel (17) vorliegt, zu geben als die Darstellung bei Růzička, der die - wohl zu verstehen als besondere - "semantische Funktion des Gerundiums" zu dessen formalen Besonderheiten in Beziehung setzt. Der Beschreibungszusammenhang, der die Strukturen in (74) wie auch (17) plausibel motiviert, ist aus prädikatenlogischen Untersuchungen $\mathrm{zu}$ Modaladverbialen gewonnen worden; z.B. legt Bartsch $^{12}$ in ihren Untersuchungen dar, daB die sprachlichen 
Strukturen die besonderen Verhältnisse widerspiegeln, die die modale Prädikation charakterisieren.

Im Unterschied zu der Prädikation durch lokale oder temporale Adverbiale setzt die modale Prädikation die Affirmation von Präd ${ }_{1}^{1}$ voraus. Nur wenn diese Voraussetzung gegeben ist, kann Präd ${ }^{1}$ in anderer Hinsicht (als der Affirmation) durch das Modaladverb näher bestimmt werden. Eine linguistisch bekannte Implikation dieser logisch-semantischen Fakten ist, daB Modaladverbien nicht "sein" modifizieren können. In der "Kopulafunktion von 'sein' ist nur eine Modifizierung hinsichtlich der Genauigkeit der Prädikation oder der Einschränkung der Prädikation möglich" (Bartsch (1972), 147). Modaladverbiale "sind nur auf solche Verben anwendbar, die außer diesen ganz allgemeinen Funktionen von 'sein' und anderen Verben bei der Satzbildung noch andere Komponenten, gleichsam Koordinaten, enthalten, die durch die modalen Adverbiale näher bestimmt werden können" (ibid. $147 \mathrm{f}$ ).

Der beschriebene Verlauf des Negations-Tests mit der ausschließlichen Verneinung des Adverbials läßt sich bei allen modalen Adverbialen beobachten, unbeschadet sonstiger formaler und eventueller struktureller Unterschiede zwischen ihnen; man vgl. die Beispiele von oben (1) - (4).

(18) na kotoruju vy ne smotreli s sožaleniem ... $\longleftrightarrow$ na kotoruju vy smotreli ne s sozaleniem ...

(19) ona ne posmotrela na menja $v$ udivlenii $\ldots \longleftrightarrow$ ona posmotrela na menja ne $v$ udivlenii ...

(20) On ne perebil ego s prezritel'noj nebrežnost'ju. $\longleftrightarrow$ On perebil ego ne s prezritel'noj nebreznost'ju.

(21) Plachi ne stojali $v$ bezmolvnom ozidanii. $\longleftrightarrow$ Plachi stojali ne $v$ bezmolvnom ožidanii. 
Dieser Verlauf des Negations-Tests trifft auf nichtkontrastive Außerungen $z u$. In kontrastiven Außerungen kann das Prädikat in dem Sinn verneint werden, daß nicht dieses durch das Modaladverb näher beschrieben wird, sondern ein anderes Prädikat ${ }^{13}$, 2.B.:

(22) Plachi ne stojali v bezmolvnom ožidanii, a ležali (v bezmolvnom ožidanii).

Da der Negations-Test bei allen Modaladverbien auch mit formal unterschiedlichen PP gleich verläuft, kann er zur Disambiguierung homonymer PP-Konstruktionen , die unterschiedlichen Adverbialklassen angehören, dienen. So ist der Satz (23) ambig:

(23) On Ženilsja po prikazaniju otca.

Die PP kann 1. die Nominalisierung eines Kausalsatzes sein:

(24) On Zenilsja, potomu そto otec èto prikazal.

Bei dieser Lesart können sowohl das Prädikat des Matrixsatzes als auch die PP negiert werden (somit werden Sätze mit unterschiedlicher Bedeutung gebildet):

(25) On ne Ženilsja, potomu と̌to otec èto prikazal. $\Longrightarrow$

On ne Zenilsja po prikazaniju otca.

(26) On Ženilsja ne potomu, tto otec èto prikazal. $\Longrightarrow$ On Ženilsja ne po prikazaniju otca.

Die PP kann 2. Nominalisierung eines Modalsatzes sein:

(27) On Ženilsja tak, kak prikazal otec. $\Longrightarrow$

Die verneinten Sätze: 
(28a) On ne Zenilsja po prikazaniju otca.

(28b) On Zenilsja ne po prikazaniju otca.

haben die Bedeutung

(28) On ženilsja ne tak, kak prikazal otec.

Neben den Modaladverbialen ist der beschriebene Verlauf des Negations-Tests noch bei den graduierenden, instrumentalen und kooperationalen Adverbialen $z \mathrm{u}$ beobachten.

Der Negations-Test ist damit geeignet, nicht nur der Unterscheidung von obligatorischen und fakultativen Adverbialen zu dienen, sondern es lassen sich damit auch sprachlich relevante spezifische Relationen einzelner subklassen der fakultativen Adverbiale $z u$ den übrigen Satzgliedern feststellen.

Es soll nun die erste der oben gestellten Fragen wieder aufgenommen werden, nämlich nach eventuellen verschiedenen Bedeutungsstrukturen innerhalb der Klassen der Modaladverbiale. 
5.2 MODALADVERBIALE VOM TYP (VP $v$ NP $\left.{ }_{\text {präp }}\right): \operatorname{Mod}_{1}$

Für die Diskussion zu den Sätzen (1) - (4) werden diese noch um die folgenden ergänzt:

(29) ... on ... voskliknul v duševnoj nemošči:

- Bat'ko, gde ty?

(30) Est', est' rekord! - vozlikoval trener Prokin i $\checkmark$ neuemnom poryve brosilsjja k svojemu vospitanniku.

(31) Lavreckij ... sidel. ... v ocepenenii otarovanija.

(32) ... obraz Lizy vosstal $v$ jego duSe vo vsej svoej krotkoj jasnosti.

(33) Okolo ... kamina rasstavleny kresla ... v živopisnom por jadke.

Sind die bei den Modaladverbialen in den Sätzen (1) - (4), (29) - (33) vertretenen Formen, nämlich die PP ( $v$ NP , ) und

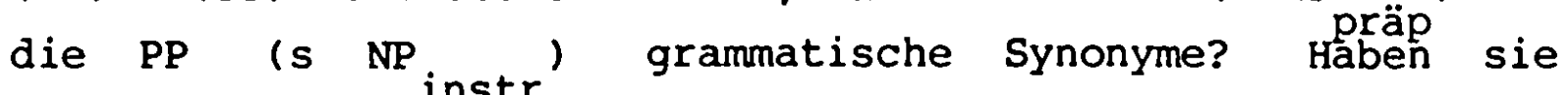
identische Relationen zu den übrigen Satzgliedern? Kommt den Sätzen damit unter diesem Gesichtspunkt die gleiche Bedeutungsstruktur zu?

Mit Hilfe einer oberflächenstrukturellen Analyse kann man eine Reihe interessanter Beobachtungen machen, die jedoch $z \mathrm{u}$ widersprüchlichen Ergebnissen auf die gestellte Frage: synonyme Konstruktion - ja oder nein - führen. Man vgl.:

es lassen sich z.B. - bei Berücksichtigung von Wortbildungsregelmäßigkeiten - die PP in allen Sätzen durch Adverbien und/oder Adverbialpartizipien ersetzen: vgl. zu (1) ... vy smotreli na nee sožaleja i ljubja ee; zu (2) ... ona posmotrela na menja udivljajas' und posmotrela udivlenno; zu (3) perebil nebrežno; zu (4) plachi stojali ... točno ožidaja そego-to ; zu (32) ... vosstal jasno; zu (33) byli rasstavleny živopisno, usw.

Dagegen führt die wechselseitige Substitution der ganzen PP - 
( $v$ NP präp durch $s \mathrm{NP}_{\text {instr }}$ und umgekehrt) - zu widersprüchlichen Ergebnissen: für (1) und (2) erhalten wir akzeptable Sätze:

(34) Ona posmotrela na menja s udivlenlem.

Nach Auskunft von native-speaker-Informanten ist bedingt akzeptabel auch:

(35) ... na kotoruju vy smotreli v sožalenii ...

Die entsprechende Substitution ergibt dagegen für (3) einen falschen Satz:

(36) ^... perebil jego Panšin v prezritel'noj nebrežnosti. usw.

Als wichtige Ergebnisse des Substitutionsverfahrens erhalten wir:

1. Nur in wenigen Fällen ist die ganze PP substituierbar.

2. In einer vielzahl von Fällen können die Präpositionen " $v$ " und " $s$ " substituiert werden unter der Bedingung des gleichzeitigen Ersatzes der NP der PP, z.B. für (3):

(37) On perebil jego $v$ rassejannosti.

oder für (32):

(38) Obraz Lizy vosstal so vsej svoej jastnost'ju.

Intuitiv verstehen wir, daB wir in (37) und (38) Sätze einer anderen Struktur als der des Ausgangssatzes erhalten haben: (37) hat transformationelle Beziehungen $2 \mathrm{u}$ :

(39) Rassejannyj, on perebil ego.

und in (38) bezieht sich "so vsej svoej jastnost'ju" in "irgendeiner Weise" auf "obraz" (vgl. oben (4) rjady pusek s otkrytymi Zerlami). 
Wie ist nun der illustrierte Sachverhalt $z \mathrm{u}$ beurteilen? Sind die modalen PP ( $S$ NP instr $^{\text {) und ( }}$ NP ${ }_{\text {präp }}$ ) synonyme Konstruktionen und die weiteren Formen mit Adverbialpartizip und Adverb weitere synonyme Oberflächenstrukturen des modalen Adverbials? Zweifel an dieser Klassifizierung rufen z.B. die Ergebnisse der ungramnatischen Sätze (36) wie auch

(40) *Plachi stojali s bezmolvnym ozidaniem. hervor. Es liegt hier offensichtlich keine semantische Inkompatibilität $z$ wischen den Nomen der PP und dem Verb vor, denn man kann das $\mathbf{N}$ durch andere Substantive substituieren und das Ergebnis ist wiederung ein unkorrekter Ausdruck: *stojat' s pročnost'ju; *stojat' s odinočestvom.

Weitere Beispiele, bei denen das Verb in der Regel nicht durch eine modale PP in der Form (s NP instr) erweitert werden kann, sind:

Zit' $v$ odinožestve, $v$ družbe $s$ kem-to ${ }^{19}$

$\underline{\text { Zit' }} \mathrm{v}$ bednosti, $\mathrm{v}$ gore, $\mathrm{v}$ radosti, $v$ nuzde

umirat' $v$ odinoðestve

sidet' $v$ ožidani $i$

lezat' $v$ razvalinach

raspolagat' Cem-to $v$ (ne)dostatke

(vgl. Grammatika (1970) 533).

Aus diesen Ergebnissen kann man schlußfolgern:

1. daß die PP (s NP ${ }_{\text {instrr }}$ ) und ( $v$ NP ${ }_{\text {präp }}$ ) in den Sätzen (1) (4) , (29) - (33) keine grammatischen Synonyme sind;

2. daß die oberflächenstrukturelle Analyse auf die Unterschiede dieser Konstruktion zwar aufmerksam machen kann, daß sie diese aber nicht beschreiben kann.

Auf der Suche nach in der Sprache sich manifestierenden zusammenhängen, die die Tiefenstruktur der obigen Sätze verdeutlichen könnte, habe ich das schon erwähnte Ergebnis 
verfolgt, daß nämlich bei Ersatz der NP der PP die Konstruktionen "s NP instr" und "v NP " für die Mehrzahl der Fälle substituierbar sind, wir gann aber - wie wir intuitiv erkennen - Sätze einer anderen struktur erhalten. Wenn wir in diesem zusammenhang in Betracht ziehen, daß es sich bei den zur Diskussion stehenden PP um freie Adverbiale handelt, d.h. nach unserer tiefenstrukturellen Analyse um abgeleitete Sätze, so liegt die Vermutung nahe, daß es sich bei den PP ( $V$ NP präp $_{\text {) und (s NP }}$ instr) um modale Prädikationen handelt, für griëp die Unterschiede in unterschiedlichen Relationen zu den übrigen Satzgliedern, im besonderen zu Präd, das sie modifizieren, $z u$ suchen sind. Für die strukturelle Analyse dieser Unterschiede reicht der Prädikations-Test nicht aus. Dieser muß für die Bestimung von Subklassen der Modaladverbiale durch weitere operationelle Tests ergänzt werden. In der folgenden Darstellung russischer Modaladverbiale lehne ich mich an die Untersuchungen von Bartsch (1972, $149 \mathrm{ff}$ ) an. Sie entwickelt Tests, die unterschiedliche Variablenbereiche für die Prädikate feststellen, über die die Modaladverbiale eine Aussage machen. In diesem Sinn spreche ich im ff von Prädikatsvariablen und Prädikatsvariablen-Tests. Als für die Subklassifizierung modaler Adverbiale relevante Variablen nennt Bartsch a) "VORGANG/ZUSTAND" und b) HANDLUNGSVOLLZUG. Wenn sich die von Bartsch entwickelten Analysen prinzipiell auf das Russische anwenden lassen, so könnte es sich auch hier - wie wir schon mehrmals bei den Adverbialen beobachten konnten - um übersprachlich relevante Bedeutungsstrukturen von Sätzen handeln.

Als heuristische Untersuchungsprozeduren schlage ich für die Prädikatsvariablen-Tests im Russischen folgende Formulierung vor:

a) für die Variable "VORGANG/ZUSTAND"

(40) Oni Zili v radosti.

Kak oni zili, bylo radostno. 20 
b) für die Variable "HANDLUNGSVOLLzUG":

(3) Panšin perebil ego s nebrežnost'ju. Kak Panšin perebil ego, proizošlo nebrežno.

Wir beginnen die Uberprüfung der Belege mit modalen PP mit denen, die die Form ( $v$ NP , ) haben. Wenden wir auf (40) den Test b) an, erhalten wir einen ungrammatischen satz:

^Kak oni žili, proischodilo radostno.

Das unterschiedliche Testergebnis erlaubt den SchluB, in der PP ( $v$ NP , ) eine bestimmte Klasse modaler Adverbiale zu sehen, die im Folgenden mit Mod ${ }_{1}$ gekennzeichent wird. Man vgl. weiter:

(41) Vse vesennie sobytija క̌li v strogoj posledovatel'nosti. Rasstajal sneg. Prileteli pervye pticy ... Kak కli sobytija, bylo strogo posledovatel'no. *Kak కli sobytija, proischodilo posledovatel'no.

(33) Okolo kamina byli rasstavleny kresla... v Živopisnom porjadke.

Kak byli rasstavleny kresla, bylo Zivopisno.

*Kak byli rasstavleny kresla, proizošlo Zivopisno.

entsprechend: (es wird im Folgenden in der Regel nur Test a) angewendet):

(42) On stojal u zatoplennogo kamina, založiv ruki za žilet, v Zivopisnom položenii ...

Kak on stojal ..., bylo v Živopisnom položenii...

(4) Plachi stojali $v$ bezmolvnom ožidanii ...

Kak plachi stojali, bylo $v$ bezmolvnom ožidanii.

(31) Lavreckij ... sidel ... v ocepenenii ołarovanija.

Kak Lavreckij sidel, bylo $v$ ocepenenii ołarovanija.

(32) Obraz Lizy vosstal v ego duše vo vsej svoej krotkoj jasnosti. 
Kak obraz Lizy vosstal $\mathbf{v}$ ego duše, bylo vo vsej svoej krotkoj jasnosti.

(43) Doma ležali v razvalinach.

Kak doma lezali, bylo $v$ razvalinach.

Im folgenden Beispiel ist die PP Mod mit einem Modaladverb durch die Konjunktion "und" verbunden:

(44) Budet polezno periodiCeski i v objazatel'nom porjadke stavit' na rassmotrenie kollektivov otčety rukovoditelej

Kak stavili na rassmotrenie otCety, bylo $v$ objazatel'nom porjadke.

(45) ... slyకił', kak krugom ... kružitsja v Žiznennom vichre ljudskaja tolpa.

Kak kruzilas' ljudskaja tolpa, bylo $v$ žiznennom vichre.

Vgl. auch eine weitere Oberflächenrealisierung nach Nominalisierungs-Tf:

(45a) KruZenie list'ev bylo vichrevym.

(46) Cuvsturues', Zto fantazija nakonec ustaet, istošaetsja $\checkmark$ večnom naprjaženii.

Kak istostalas' fantazija, bylo $v$ večnom naprjaženii.

(47) Prazdnik byl velikolepnyj ... vina ja togda esče $v$ rot ne bral, $v$ chorovodach chodit' ne umel.

Kak ja chodil, bylo $v$ chorovodach.

Gestützt auf die Hypothese, daß auch die modalen Adverbiale tiefenstrukturell Prädikationen sind, wird versucht mit Hilfe der Prädikatsvariablen-Tests die satzsemantischen Merkmale, die diese Prädikation auszeichnen, explizit zu machen.

Die russischen Sätze mögen in der Metasprache dieser Tests zum Teil stilistisch ungewöhnlich sein; dennoch machen die Operationen deutlich, daß 1. Test b) mit der Prädikatsvariablen "HANDLUNGSVOLLZUG" über die in den analysierten Sätzen vertretenen Verben (Präd) keine zutreffende Aussage 
macht 22 , da das aus dem Adverbial $\left(\bmod _{1}\right)$ gebildete Prädikat, das nun das Prädikat des Matrixsatzes näher bestimmt, auf dieses nicht zutrifft. Das hängt damit zusammen, daß - wie die Beispiele zeigen - diese Klasse Verben sich in ihrer satzsemantischen (und hier auch wortsemantischen) Bedeutung durch die Merkmale (Zustand/Vorgang) auszeichnet und für sie damit eine Prädikation mit einem Verb, das über "Handlungsvollzüge" prädiziert, hier konstruiert mit "proischodit'", nicht stimmt.

Argumentieren wir vom Standpunkt der Erweiterungsmöglichkeiten von Sätzen mit Modaladverbialen des Typs PP (v NP präp', so können die bisherigen Testergebnisse folgendermaßen zusammengefaßt werden: Test a) führt die PP auf einen Satz zurück, der eine Aussage über das "wie" eines "Vorgangs" oder "Zustands" macht - diese Merkmale der semantischen Matrix von Präd ${ }^{1}$ (VP) sind für die Erweiterungsmöglichkeiten von Sätzen mit Mod ( ${ }_{1}$ NP ${ }_{\text {präp) }}$ ) relevant. Mit folgenden Ersetzungsregeln sollen die Ergebnisse vorläufig formalisiert werden:

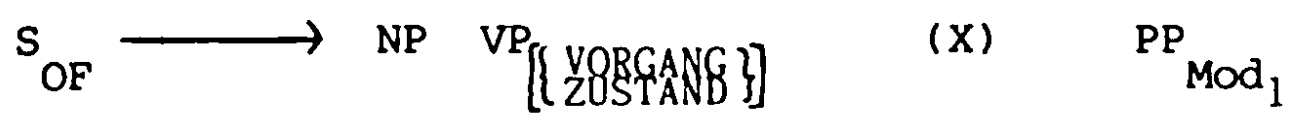

$\mathrm{MPd}_{1} \longrightarrow \quad \mathrm{N} \quad \mathrm{NP}_{\text {präp }}$

Für die Beschreibung der Sätze mit Mod habe ich bisher aus methodischen Gründen solche Beispiele gewählt, deren Prädikat in seiner lexikalischen Bedeutung eine relativ einfache semantische Merkmalstruktur 23 hat: "stojat'", "ležt'", "sidet'" usw., die in ihrer allgemeinen Bedeutung "Zustände" bezeichnen, und "zit'", "umirat'", "istołat'sja", "kruZit'sja" usw., die "Vorgänge" bezeichnen. Bei Verben mit komplexerer semantischer Merkmalstruktur wie oben (1, 29, 30) und die folgenden Beispiele $(48-54)$ ist bei einer Analyse modaler PP eine klare Unterscheidung zwischen einer Untersuchung auf satzsemantischer und/oder wortsemantischer Ebene 
geboten. Für die satzsemantische Analyse ist zu fragen, welches der wortsemantischen Merkmale des verbs in der Beziehung zu der PP - d.h. im Hinblick auf die wahl zwischen Mod und Mod ${ }_{2}$ - aktuell ist. Wenn es die semantischen Merkmale (Vorgang) oder (Zustand) sind, so sind in der Regel die Voraussetzungen für eine Erweiterung mit Mod gegeben (zu den Regeln für Erweiterungen mit anderen PP ${ }_{\bmod }$ s.s. $209 \mathrm{ff}$ ). Aus diesen zusammenhängen folgt, daß man fǘd diese verben keine disjunkten Klassen hinsichtlich ihrer Prädizierbarkeit von Modaladverbialen aufstellen kann. Andererseits ist bei den Verben wie "sidet'", "Žit" etc. die wortsemantische Merkmalstruktur von der Art, daB sie in der Regel nur durch Modaladverbiale vom Typ Mod, d.h. für PP-Konstruktionen durch ( $v$ NP $)$, erweitert werden können. Insofern bilden sie eine disjunkte Klasse im Verhältnis zu anderen Verben.

Beispiele mit Mod, in denen Präd ${ }^{l}$ von Verben mit komplexer Merkmalstruktur besetzt sind, so dab sie auch von der Prädikatsvariablen "HANDLUNGSVOLLZUG" prädizierbar wären, was aber in dem angegebenen Text nicht relevant ist, waren oben (29) und (30). (Es wird hier nur Test a) angewendet; die Anwendung von Test b) in Sätzen mit vergleichbaren Verben, erfolgt unten S. $208 \mathrm{fl}$ ):

(29) ... on ... voskliknul v dusevnoj nemosti ...

Kak on voskliknul, bylo $v$ dusevnoj nemosti.

(39) ... Pronkin ... v neuemnom poryve brosilsja $k$ svoemu vospitanniku.

Kak Pronkin brosilsja $k$ svoemu vospitanniku, bylo $\mathrm{v}$ neuemnom poryve.

Weitere Beispiele sind:

(48) Son byl chorošo snjat $v$ cvete na plenke Kodak.

(49) Tam idet uborka $v$ gruppach.

(50) Vse delaetsja kak vsegda. Pereodevanie. Domasnie tufli. 
Obed v moltanii. Esli èto možno nazvat' obedom.

(aus Zolotova, 1973, 262)

(51) ... progovoril on $v$ blagorodnom negodovanii.

(52) ... vskri $x_{a l}$ Mozgljakov $v$ neobyknovennom volnenii.

(53) ... vskrical on $v$ bešenstve.

(54) ...v istuplenii vskriðala Mar'ja Aleksandrovna.

(55) ... povtoril on $v$ neterpenii.

Unter den Belegen überwiegen solche, in denen die Verben (Präd ${ }^{1}$ ) psychische und/oder geistige Handlungen bezeichnen. Die Sätze (29), (51 - 55) können als homonyme struktur zu Sätzen verstanden werden mit PP ( $v$ NP ), in denen sich die PP jedoch auf das Satzsubjekt bezieht (S. dazu S. $204 \mathrm{ff})^{24}$. Die Bewertung der PP ( $v$ NP präp) als homonym halte ich für plausibel, da sich zum einen die tiefenstrukturelle Analyse für Mod auf diese Sätze anwenden läßt, und zum anderen, da die PP ${ }^{1}$ in $(29,51$ - 55) syntaktisch synonym - in dem von uns definierten Sinn (s.o. S. $38 \mathrm{f}$ und S. 51) - zu folgender Oberflächenstruktur ist: vskričal vzvolnovanno, beక̌eno, istuplenno; povtoril neterpelivo.

Für die Konstruktion (VP $\vee$ NP präp) sind auch solche Ausdrücke typisch, in denen die PP aus ${ }^{\text {präp } A ~ N) ~ b e s t e h t ~(v g l . ~(33), ~}$ (42)). Dabei kann $N$ ein Füllwort sein, die Prädikation geschieht mittels $A$ :

(56) Mestoimenie "kto" upotrebljaetsja v voprositel'nom značenii.

Mestoimenie "kto" upotrebljaetsja voprositel'no. Kak mestoimenie "kto" upotrebljaetsja,- voprositel'no.

Eine Tilgung von A ergibt einen ungrammatischen Satz: «Mestoimenie "kto" upotrebljaetsja $v$ znaCenii.

(57) ... gospodin ... protestoval protiv menja $v$ dovol'no ènergičeskich terminach. 
... gospodin protestoval protiv menja dovol'no ènergi ̌̌no.

Kak ... gospodin protestoval protiv menja, bylo dovol'no ènergično.

*... gospodin ... protestoval protiv menja $v$ terminach.

(58) Begstvo ot sovetskoj real'nosti ... v naibolee ubeditel'nom vide možno ... nabljudat' u Solouchina. Begstvo ot sovetskoj real'nosti ... naibolee ubeditel'no možno ... nabljudat' u Solouchina. Kak možno (bylo) nabljudat' begstvo ot sovetskoj real'nosti, (bylo) ubeditel'no.

*Begstvo ot sovetskoj real'nosti ...v vide možno ... nabljudat' u Solouchina.

Weitere Beispiele:

(59) Sistema $\mathrm{v}$ polnoj sile svoej primenena byla tol'ko $\mathrm{k}$ Fede.

Sistema polnost'ju primenena byla tol'ko $k$ Fede.

(60) vyskazat'sja ob ètom $v$ ironičeskoj forme; vyskazat'sja ironið̌eski; *vyskazat'sja v forme.

(61) napisat' kartinu $v$ iskusnoj forme; napisat' kartinu iskusno; *napisat' kartinu $v$ forme.

Dieser Ausdruck von Modaladverbialen hat syntaktisch und wortsemantisch eine Reihe gemeinsamer Merkmale mit Modaladverbialen im Instrumental ohne Präposition:

(62) Nu ¿to-z, skazal on ravnodusnym tonom.

(63) Lesa suetilis' rozovym cvetom.

Wie ich an anderer Stelle 25 zeigen konnte, ergibt die Tilgung von A einen ungrammatischen Satz und verlangen die Selektionsregeln, nach denen diese Konstruktion (VP NP ${ }_{\text {instr) }}$ ' gebildet wird, eine enge semantische Merkmalentsprechung von VP und $\mathbf{N}$ : 
On skazal

lesa svetilis'

$$
\left[\begin{array}{l}
\text { visuell } \\
\text { realisieren }
\end{array}\right]
$$

rozovym cretom

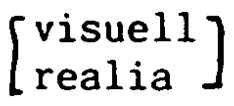

Die Bildungsmöglichkeit dieser Konstruktion ist aufgrund der aufgezeichneten Regeln, denen die Relationen der Satzglieder untereinander genügen müssen, beschränkt, so daß es berechtigt sein dürfte, diese Ausdrücke als lexikalisiert $z u$ bezeichnen.

Eine weitere Gruppe von Beispielen mit PP als eine Art modaler Prädikation durch ( $V$ NP ) bilden solche, bei denen die Prädikation durch ein Prädikä der Form ("sein" NP ..) erfolgt. Für diese sätze ist charakteristisch, daß die $\mathbf{N}^{\mathrm{a}}$ mit Nomina besetzt ist wie "kacestvo", "rol'" etc., d.h. Nomina, die vergleichende Attribute ausdrücken:

(64) Dokument upotrebljaetsja $v$ kałestve diploma. Kak on upotrebljaetsja, -[byt' diplomom].

(65) Dannoe slovo upotrebljaetsja $v$ roli opredeleni ja imeni. Kak dannoe slovo upotrebljaetsja, $-[$ byt' opredeleniem imeni].

Fassen wir die bisherigen Ergebnisse der Analyse russischer Modaladverbiale zusammen:

die überwiegende Menge der Modaladverbialen sind fakultative Ergänzungen im Satz, die das Verb näher bestimmen. Insoweit gleichen sie den temporalen Adverbialen und lassen sich entsprechend - unter Berücksichtigung der Prädikation als modale - mit Hilfe des Prädikations-Tests auf Sätze zurückführen. Für eine schematische Darstellung der Abhängigkeitsrelationen von $S^{1}$ TS und $s^{2}$ TS können wir auf das in diesem Zusammenhang vorgeschlagene Schema 3a zurückgreifen, s. oben S. 141 (die Ableitung von $\mathrm{S}^{2}$ TS ist vereinfacht). Man vgl.: 
STS

zu $(40):$ Oni
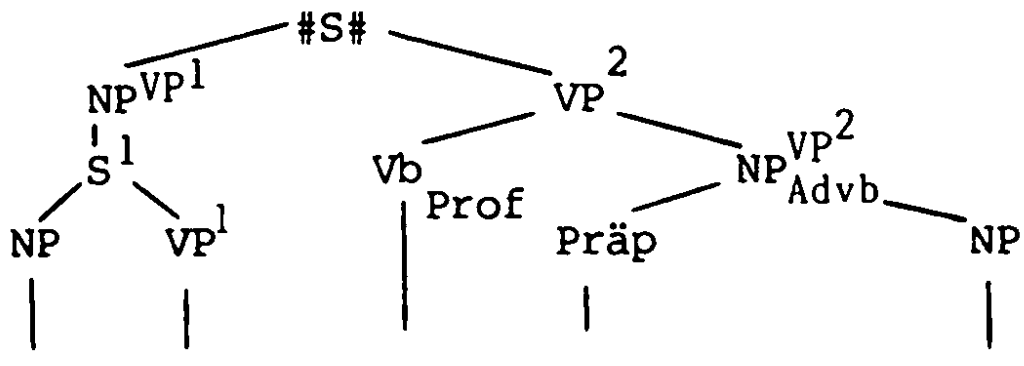

Zili

$\theta$

v

radosti.

Andererseits stellen wir strukturelle Besonderheiten in Sätzen mit Modaladverbialen fest, die durch die modale Prädikation bedingt sind: der Negations-Test, angewendet auf die 3 hier untersuchten Adverbialklassen, zeigt, daß sich die Modaladverbiale dadurch von den fakultativen Lokal- und Temporaladverbialen unterscheiden, daß nur die Prädikation von Präd über Präd, d.h. die damit behauptete Relation, verneint werden kann, nicht aber der Sachverhalt von Präd . Positiv ausgedrückt: die wahrheit von Präd ist Voraussetzung für die Erweiterung von Sätzen mit Modaladverbialen.

Weiter führten uns die Beobachtungen an Sätzen mit Modaladverbialen $z u$ dem Nachweis von Subklassen innerhalb der Klasse der Modaladverbiale. Mit Hilfe der Prädikatsvariablen-Tests ließen sich oberflächenstrukturell unterschiedliche Formen der PP und die Regeln zu Erweiterung von Sätzen mit ihnen durch eine Differenzierung der satzsemantischen verhältnisse motivieren. Bisher wurde die Subklasse Mod der Modaladverbiale untersucht (als PP in der Form: $v$ NP 1 ), deren sie auszeichnendes satzsemantisches Merkmal darin präp besteht, daß sie eine qualifizierende Aussage über Vorgänge und/oder zustände, ausgedrückt durch Präd, macht; das wurde metasprachlich festgehalten durch die Prädikatsvariable "VORGANG/ZUSTAND".

Bevor die Analyse weiterer Subklassen der Modaladverbiale aufgenommen wird, soll in Ergänzung zu Mod und zur Unterscheidung von diesen die Prädikation des satzsubjekts zur Sprache kommen, die formal auch mit Hilfe der PP ( $V$ NP präp' erfolgt. 


\subsection{PRÄDIKATION DES SATZSUBJEKTS/-OBJEKTS}

Von der Satzstruktur mit Mod ${ }_{1}$ sind Sätze wie folgende zu unterscheiden:

(66) Ja brodil po gorodu v glubokoj toske.

(67) V smertel'noj toske vozvraščalsja ja $k$ sebe domoj. ${ }^{28}$

Die Verwendung der PP kann ambig sein, da sie sich auf das Satzsubjekt und -objekt beziehen kann:

(68) Ja videl ee $v$ smertel'no bol'nom sostojanii.

Sätze dieses Typs stehen in transformationeller Beziehung $z u$ folgenden:

(69) Gluboko tosklivyj, brodil ja po gorodu. 29

(70) Smertel'no tosklivyj, vozvrašcalsja ja $k$ sebe domoj.

Eine Tf mit Adverb ergibt in der Regel einen ungrammatischen Satz:

(71) *Ja brodil po gorodu tosklivo.

(72) *Ja vozvraščalsja $k$ sebe domoj tosklivo.

Die syntaktischen Relationen der Satzglieder verdeutlichen, daß die PP ( $V$ NP präp) in diesen Fällen das Subjekt des Satzes näher beschreiben, d.h. zu der Klasse folgender Sätze zu rechnen ist:

(73) ... ja ešče do sich por ne $v$ silach byl povidat'sja s izurodovannym moim bednjakom.

(74) Ja v vostorge. 
Für die schematische Darstellung der syntaktischen Relationen der Sätze wie (69), (70) schlägt Růžička (1970a) einen Stammbaum vor (loc. cit. 252), den ich bei geringfügigen Abänderungen für geeignet halte, nicht nur die struktur der Sätze vom TYp (66), sondern auch die Unterschiede zu Sätzen mit Mod $2 u$ verdeutlichen (vgl. oben Schema $3 a)$ :

Schema 4:

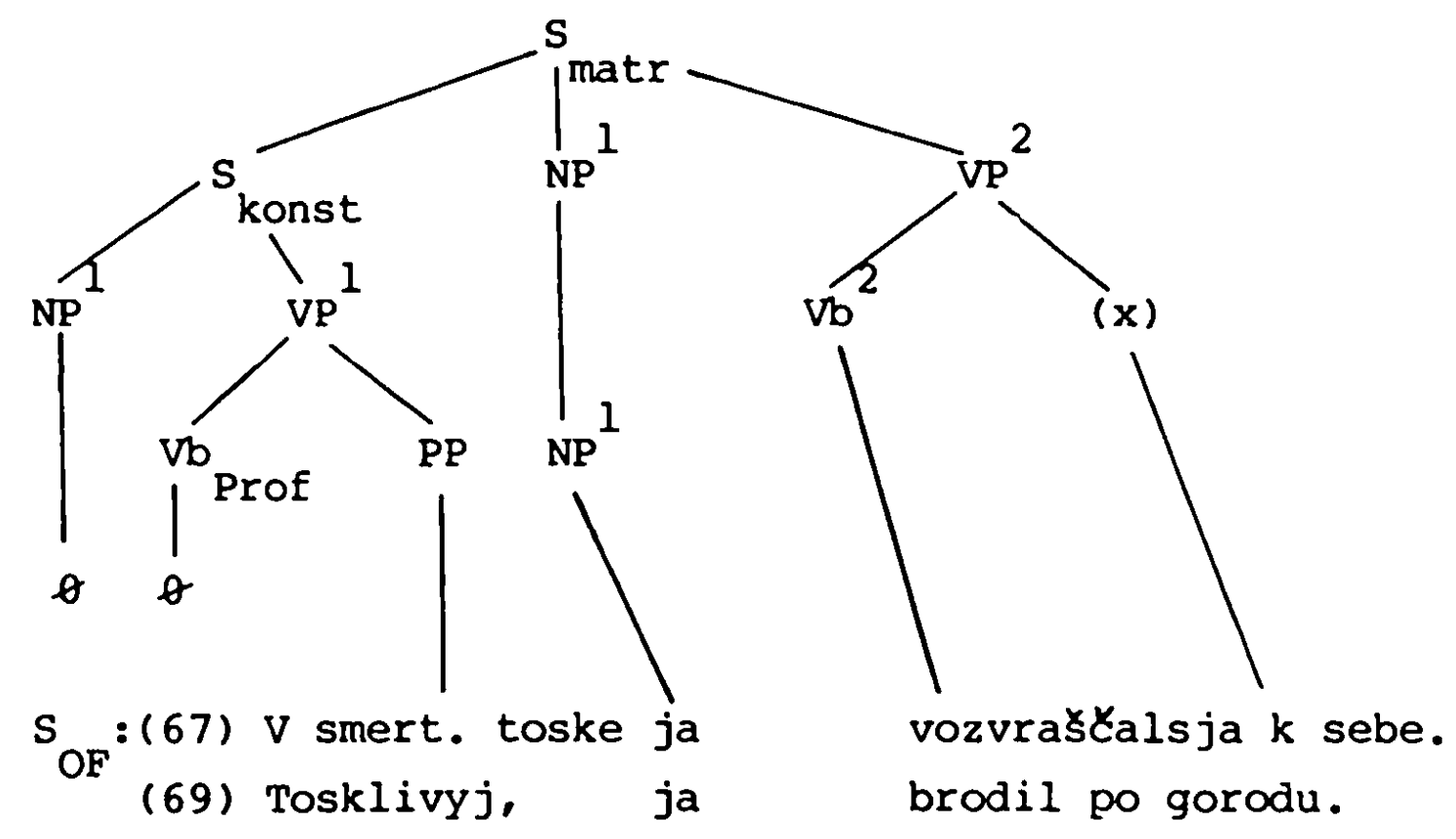

Die Blockierung der Adverb-Tf in Sätzen wie (66), (67) - vgl. (71), (72) - ist wohl damit verbunden, daß die semantischen Merkmale des $\mathbf{N}$ der PP für eine Prädikation des Verbs des Matrixsatzes nicht passen: der Vorgang einer konkreten Bewegung (z.B. des Gehens) wird in der Regel nicht mit "traurig sein", "schwermütig sein" bezeichnet, sondern diese Aussage bezieht sich auf NP (vgl. auch Stammbaum). Gehört dagegen Präd ${ }^{1}$ zu der Verbklasse, die psychische und/oder geistige Handlungen bezeichnet, können die Beziehungen der PP ( $v$ NP präp' ambig werden; wir haben dann den Fall der oben beschriebenen Homonymie; vgl. im Unterschied zu (67):

(75) Gospodi - dumaet on $v$ nevynosimoj toske ${ }^{30}$ - chot' zachvorat' by me. 
Die Adverb-Tf ist hier bedingt zulässig:

(75a) ... - dumaet on tosklivo.

Die explizite Bedeutung dieses Ausdrucks besagt, das "denken" als geistiger Vorgang durch eine psychische Eigenschaft "traurig sein", näher bestimmt wird; vgl. "mysli tosklivye".

Die Untersuchungen zu PP ( $v$ NP präp') als Mod $_{1}$ oder als Prädikation des Satzsubjektes bzw. präp -objektes weisen auf ein methodologisches Problem: tiefenstrukturelle Untersuchungen z.B. in der generativen semantik lassen Kriterien für eine Relevanzbestimmung ihrer semantischen Merkmale vermissen. Im Prinzip ist die Matrix dieser Merkmale unbegrenzt. Die hier vorgelegten Untersuchungen, die sich auf breite empirische Daten und Fachliteratur mit vergleichbarem Beschreibungsansatz stützen, zeigen m.E., daß in Ergänzung der generativtransformationellen Analysen es die satzsemantische Bestimmung der Prädikation ist, von der die Regeln für die Erweiterungsmöglichkeit, bzw. Blockierung von Sätzen mit freien Adverbialen abgeleitet werden können. Die Satzsemantik untersucht die Relationen der Prädikate $z u$ ihren Argumenten (wobei die so verstandenen Prädikate und Argumente in den Oberflächensätzen ganz verschiedene "Satzglieder" und Wortklassen vertreten können). Dieses Kriterium der satzsemantischen Relationen könnte, soweit Prädikationen vorliegen, den Rahmen für das, was unter den wortsemantischen Merkmalen für die Verwendung des betreffenden sprachlichen Zeichens relevant ist, liefern. Als Beispiele können hier die Ergebnisse der Untersuchungen zu PP als Mod und Prädikation von Satzsubjekt und/oder -objekt angeführt werden: präzisierend $z u$ dem bisher beschriebenen Regelmechanismus für die Erweiterung von Sätzen mit Mod können wir in Abgrenzung zu der verwendung von PP ( $v$ NP ${ }_{\text {präp }}$ ) als Prädikation des Satzsubjekts/-objekts festhalten, ${ }_{\text {däb }}$ eine wortsemantische 
Merkmalentsprechung zwischen VP (Präd ${ }^{l}$ ) und dem $N$ der PP gegeben sein muß ( $\mathrm{s}$. Kommentar $\mathrm{zu}$ (71) und (75)). In den Ersetzungsregeln für Sätze mit Mod (vgl. S. 198) sei diese Relation mit $(y)$ vermerkt:

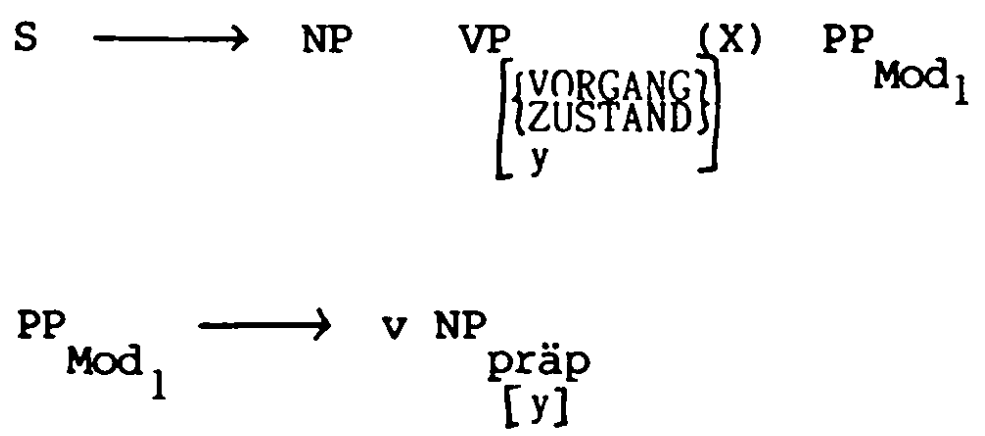


5.4 MODALADVERBIALE VOM TYP (VP s NP instr ): MOd $_{2}$

Zur abschließenden Beantwortung der Frage: ist den modalen PP

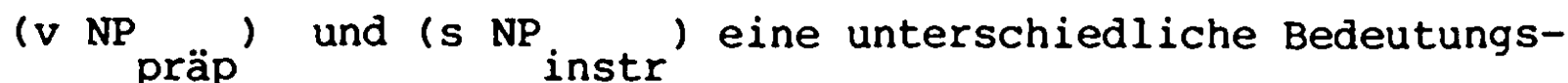
struktur zuzuschreiben, soll jetzt versucht werden, eine Beschreibung der PP ( $\mathrm{NP}$, zu geben. Im Unterschied zu den Adverbialen Mod unterliegt die Erweiterung von Ausdrücken mit PP. (s NP instr) nicht vergleichbar strengen Selektionsregeln. Das zeigt sich quantitativ darin, daß diese Konstruktion den Großteil der Belege mit modalen PP ausmacht. Wir ziehen für die Darstellung außer Satz (I) und (3) noch die Sätze (79-88) heran:

(79) ... proiznesla Liza s nebol'šoj zapinkoj.

(80) - povtorila s ukoriznoj staruška.

(81) ... probormotala s dosadoj Mar'ja Dmitrievna.

(82) Ona s usiliem pojmala ruku muza.

(83) Ona s uspechom razvodila pautinu.

(84) Ona odevaetsja so vkusom.

(85) On s rezkost'ju i neterpelivost'ju vedet svoi ataki.

(86) On k zernu priblizaetsja s chitrost'ju.

(87) - ... sprosil on $s$ volneniem.

(88) ... on ... umel s polunasmełlivoj važnost'ju

zanimat'sja vzdorom.

Aus der Kommentierung und Illustration der PP Mod folgt, daß von einer Gegenüberstellung der Anwendung der beiden Prädikatsvariablen-Tests an den Beispielen keinerlei differenzierende Aussagen $\mathbf{z u}$ dem hier gestellten Problem $z u$ erwarten sind, da über Vollverben in der Regel Aussagen über das "wie" ihres "Vorgangs" gemacht werden können. Dagegen ist eine Uberprüfung der Sätze mit dem Prädikatsvariablen-Test b) aufschlußreich: zum einen, um die Besonderheiten der modalen Prädikation in den oben genannten Sätzen deutlich zu machen, zum anderen aber auch, um Kriterien für die Beschreibung 
der PP ( $s \quad \mathrm{NP}_{\text {instr }}$ ) als modal im Unterschied $z \mathrm{u}$ ihrer Verwendung in anderen Funktionen - z.B. als kooperationales Adverbial, wie schon angedeutet - $\mathbf{z u}$ gewinnen. zur Unterscheidung von Mod wird diese Klasse modaler Adverbiale Mod $_{2}$ genannt. Für die Uberprüfungsprozedur durch den Test ist es erforderlich, die Ebene als metasprachliche zu kennzeichnen, das satzargument steht daher in [ ]. Aus Gründen, die mit WortbildungsregelmäBigkeiten ${ }^{31}$ zusammenhängen, werden die obigen Beispiele in geänderter Reihenfolge angeführt.

zu ( 3): [Kak perebil ego Panšin,] proizošlo nebrežno. zu (85): [ Kak on vedet svoi ataki,] proischodit rezko i neterpelivo.

zu (86): [Kak on približaetsja k zernu,] proischodit chitro. zu (87): [ Kak on sprosil,] proizošlo vzvolnovanno.

zu (88): [Kak on umel zanimat'sja vzdorom,] proischodilo $s$ polunasmešlivoj vaźnost'ju.

zu (79): [Kak ... proiznesla Liza,] proizoslo s zapinkoj. $z u(80):$ [Kak ... povtorila staruska,] proizoslo s ukoriznoj. $z u(81)$ : [Kak ... probormotala Mar'ja Dmitrievna,] proizošlo s dosadoj.

zu (82): [Kak ona pojmala ruku muza,] proizošlo s usiliem. zu (83): [Kak ona razvodila pautinu,] proischodilo uspexno. zu (84): [Kak ona odevaetsja,] proischodit so vkusom.

Als Ergebnis der Anwendung des Prädikatsvariablen-Tests b) auf (3), (79 - 88) können wir feststellen, daß mit dieser Subklasse der Modaladverbiale eine Aussage über das "wie" des "Handlungsvollzugs", bezeichnet durch Präd", gemacht wird. Die Ersetzungsregeln für Sätze mit $\operatorname{Mod}_{2}$ sind:

$$
\begin{aligned}
& \mathrm{S}_{\mathrm{OF}} \longrightarrow \mathrm{NP} \quad{ }_{\text {[Handlungsvollzug] }}^{\mathrm{VP}}(\mathrm{X}) \mathrm{PP}^{\prime \prime} \\
& \mathrm{MPd}_{\mathrm{MOd}_{2}} \longrightarrow \mathrm{S} \quad{ }_{\text {instr }}^{\mathrm{NP}}
\end{aligned}
$$


Als Ergebnis des Unterschieds bei der Verwendung der modalen PP ( $v$ NP präp) und ( $s$ NP instr) können wir festhalten: Bei der Präp Erweiterung von Sătzen mit ( $v$ NP Aussage über das "wie" des "Vorgangs/Zustands" päp des mit Präd bezeichneten Verbs gemacht. Im Rahmen der damit beschriebenen satzsemantischen Verhältnisse konnte eine weitere Regel, die die wortsemantischen Implikationen dieser Prädikation betrifft, formuliert werden (s.S. $206 \mathrm{f}$ ). Mit diesen Regeln werden die Unterschiede in den modalen Aussagen - durch ( $v$ PP präp' einerseits und (s PP instr' andererseits beschreibbar. Darüberhinaus läßt sich zeigen, was noch zur Sprache kommen wird, daß Mod in struktureller Beziehung zu Satzgefügen mit modalen Nebensätzen, eingeleitet mit dem Satzkonnektor "pri ètom ..." steht. Mit den aufgewiesenen Kriterien dürfte sich der Unterschied zu (s NP, ) als kooperationales Adverbial formulieren lassen, wozu jedoch entsprechende Analysen $z u$ diesem durchgeführt werden müßten.

Aufschlußreich ist der mit den Prädikatsvariablen-Tests gewonnene Erklärungszusammenhang auch für Sätze mit "bedeutungsnahen" Verben als Präd, die durch Modaladverbiale erweitert werden. Es wurden o. S. $184 \mathrm{mit}$ (1) und (2) Belege mit "smotret'" zitiert. Uber "smotret'" kann eine Aussage, die den "Handlungsvollzug" näher beschreibt, gemacht werden; vgl. $\mathrm{zu}(1)$ :

Kak vy smotreli, proischodilo inogda s zalost'ju, inogda s sostradatel 'noju l jubov'ju.

Das gilt nicht für "videt'" ${ }^{34}$ : hier ist eine Erweiterung von Sätzen mit Mod ${ }_{2}$ blockiert; vgl.:

(89) *Vse èto my videli $\left\{\begin{array}{l}s \text { vostorgom. } \\ s \text { užasom. }\end{array}\right\}$ etc.

Vgl, dagegen: 
(90) On vidit choroso. - Ona vidit tol'ko $v$ oxkach.

Die Anwendung des Prädikations-Tests macht auf eine Oberflächenstruktur aufmerksam, die der hier beschriebenen $\mathrm{PP}_{\mathrm{Mod}_{2}}$ nahe ist und die die Ergebnisse des Prädikatsvariablen-Tests bestätigt, bei der jedoch die modale Prädikation mit Hilfe eines modalen Nebensatzes ausgedrückt wird; vgl.:

(91) Vy smotreli na nee, pri ètom vy inogda zaleli, inogda ljubili ee.

(92) Panక̌in perebil ego, pri ètom on byl nebrežnym/ $\left\{\begin{array}{l}\text { vel sebja } \\ \text { dejstvoval }\end{array}\right\}$ nebrežno.

(93) ... proiznesla Liza, pri ètom ona zapinalas'.

(94) On vedet svoi ataki, pri ètom on rezkij i neterpelivyj.

(95) On umeet zanimat'sja vzdorom, pri ètom on polunašmeslivo važnyj. etc.

Das Nomen der PP wird auf ein zugrundeliegendes Prädikat zurückgeführt. Aufgrund von Wortbildungsregeln kann dabei einerseits auf Verben zurückgegriffen werden (91, 93), andererseits auf prädikative Ausdrücke, die aus ("sein A") zusammengesetzt sind (92, 94, 95). Im einzelnen können Konstruktionen entstehen, in denen die Besetzung wegen fehlender Lexeme durch Synonyme erfolgen muß. So wäre im Fall von (81) zu ersetzen: - probormotala ona, pri ètom ona byla razdražennoj; oder die Bildung ist durch wortsemantische Spezialisierungen der Lexeme blockiert (s. dazu weiterer Kommentar zu (84) S. 215 f). Der Satzkonnektor "pri ètom" soll die satzsemantischen Beziehungen zwischen Matrixsatz und Konstituentensatz verdeutlichen:

Der Konstituentensatz macht nähere Angaben über das "wie" des Prädikats im Matrixsatz. Dabei ist die NP des Konstitu- 
entensatzes in der Regel mit der des Matrixsatzes identisch $^{35 / 36}$. Es ist zu erwarten, daß sich aus diesen Beziehungen Restriktionen für die Besetzung der NP der $\mathrm{PP}_{\mathrm{Mod}_{2}}$ (etwa im Unterschied $z u$ (s NP kooperational)') ergeben - ich kann diese Frage hier nicht weiter verfolgen, da für ihre Untersuchung mindestens die kooperationalen Adverbialen mitbehandelt werden müßten.

Den beschriebenen satzsemantischen und syntaktischen Merkmalen von PP Mod ${ }_{1}$ und Mod ${ }_{2}$ entspricht, daß Verben wie "žit'", "sidet"n, "raspolagat' '̌em-to", die in der Regel "Zustände" bzw. "Vorgänge" bezeichnen, mit Mod (s NP ${ }_{2}$ instr') nicht erweitert werden können. Das heist aber nicht ${ }^{2}$ - wie schon erwähnt- , daß die Verben, die "üblicherweise" mit Mod erweitert werden $(1,3,79-88$ ) nicht mit Mod ( 1 NP präp) näher beschrieben werden können. "Opisat'" ist auch als "Vorgang" (Beschreib-Vorgang) darstellbar; vgl.:

(96) Potebnja opisal ètu strukturu v obstich Łertach.

und auch als "Handlungsvollzug":

(97) On opisal ètu složnuju strukturu s ubeditel'nost'ju.

Es sind Sätze denkbar, in denen beide satzsemantisch relevanten Merkmale aktuell sind:

(98) On opisal strukturu, chotja i v obš̌ich ðertach, no s ubeditel 'nost' ju.

(99) On opisal strukturu $v$ podrobnych đertach i s ubeditel'nost'ju.

(100) On opisal strukturu vo vsej podrobnosti is ubeditel'nost'ju.

In der Form der Adverbien sind die unterschiedlichen Relationen von Mod, und Mod, zu dem Prädikat neutralisiert: 
(101) On opisal strukturu podrobno i ubeditel'no.

Die Untersuchungen $z u$ den unterschiedlichen satzsemantischen Funktionen von Mod und Mod ermöglichen, Regeln für die Erweiterung von Sätzen mit ${ }_{\mathrm{PP}}^{2}$ mod $z$ formulieren, und machen die Blockierung von Sätzen wie

(102) *Vse krutilos' s bešenym vichrem.

(103) *On opisal strukturu s obsčimi čertami.

voraussagbar, nämlich aufgrund der gegenseitig - bei Prädikat und PP - regelwidrigen Relationen:

In (102) wird mit Hilfe des Modaladverbials über den "Vorgang" "krutit'sja" prädiziert. Diese Funktion erfüllt im Russischen nach den hier dargelegten Ergebnissen im Falle von PP in der Regel ( $V$ NP . ). In (103) ist der Regelmechanismus für die Bildung von Prạ̈t zen mit PP Mod ${ }_{1}$ oder Mod ${ }_{2}$ in anderer Hinsicht nicht erfüllt: die PP (s NP 1 , ) als $P P_{\text {mod }}^{2}$ hat die Funktion, einen "Handlungsvollzug" näher zu beschreiben; "s obsčimi certami" ist aber auf kein Prädikat zurückzuführen, mit dessen Hilfe der "Handlungsvollzug" "opisat"" näher beschrieben werden könnte.

Neben der o. S. 209 konzipierten Grundform von Sätzen mit Mod sind Fälle zu beobachten, in denen sie aufgrund von syntaktischen oder lexikalischen Faktoren verändert ist. Es handelt sich z.B. um Sätze vom Typ:

(104) Ja zametila kakuju-to chitrost' v ego postupkach. (105) Bylo לto-to rezkoe $v$ ego obrašenii s ljud'mi.

Diese Sätze bestätigen zum wiederholten Male eine methodologische Einsicht, zu der mich unter anderem meine Untersuchungen führten: Die transformationelle Analyse auch an zueinander "nahen" Oberflächenstrukturen $z u$ messen und $z u$ überprüfen. Damit eröffnet sich ein Beschreibungs- 
zusammenhang, der nicht nur zu "einfacheren" Strukturen führt, sondern auch zu "komplexeren". Als solche verstehe ich (104) und (105). Für ihre Beschreibung reichen die bisher für adverbiale PP ausgearbeiteten Untersuchungsverfahren nicht; denn danach müßte man die PP für ein Prädikat halten, das über ein anderes Satzglied oder andere satzglieder prädiziert. Eine solche Interpretation widerspricht zum einen dem intuitiven Verständnis der Sätze vom Typ (104) und ließe zum anderen die Frage unbeantwortet, ob es eine Relation von (104) $\mathrm{zu}$

(106) Ja zametila, Cto on postupal s chitrost'ju.

und von (105) $2 u$

(107) On s rezkost'ju obrašcaetsja s ljud'mi.

gibt.

Für die Ableitung von (104), (105) sind zusätzliche Transformationen notwendig, die vorrangig in einem Rollentausch von Prädiziertem und prädizierendem bestehen; man vgl.:

12

zu (106): Ego postupki chitrye.

12

On postupaet s chitrost'ju.

Dagegen ist diese Relation von (2) : (1) syntaktisch ausgetauscht in

$$
12
$$

zu (104): Cto-to chitroe $v$ ego postupkach.

Andererseits wird mit der aufgezeigten Beziehung zwischen (106) und (104) linguistisch beschreibbar, daB die PP in (104) nicht als z.B. Lokaladverbial (in figurativer 
Bedeutung) zu klassifizieren ist. Entsprechendes gilt für die Beziehung zwischen (105) und (107).

Es sind weiter Fälle zu beobachten, in denen ein transformationelles Verhältnis von Adverb $\Longrightarrow$ PP - obwohl beide Formen belegt sind - nicht vorliegt. Der Grund kann dabei in lexikalisch spezialisierten Verwendungsweisen bestimmter Lexeme liegen. Das ist m.E. der Fall bei "vkusno" und "so vkusom". "Byt" vkusnym" ist ein Prädikat mit der lexikalischen Bedeutung "schmecken" (itr). Das Adverb "vkusno" beschreibt ausschließlich solche Prädikate näher, die "Speisenverwertung" bezeichnen: "gotovit' vkusno; kormit' vkusno".

Die satzsemantischen Relationen scheinen hier auf Mod $2 u$ verweisen, man vgl.:

(108) Ėto - firmennoe bljudo, i ego gotovjat zdes' osobenno vkusnym obrazom.

Jedoch braucht man für die Beschreibung der verwendung von "vkusno" nicht auf satzsemantische verhältnisse zurückzugreifen:

Es sind wortsemantische Merkmale, die zu der Blockierung von Sätzen wie den folgenden führen:

(109) *Ona odevaetsja vkusno.

(110) *Oni obstavili kvartiru vkusno.

Komplementär zu dieser spezialisierten, nur im Hinblick auf "vkus" in der Bedeutung einer Sinnesfähigkeit des Menschen zu gebrauchenden Qualitätsbezeichnung "vkusno" wird in der Regel die PP "so vkusom" gebraucht:

(111) Ona odevaetsja so vkusom.

(112) Oni obstavili kvartiru so vkusom. 
Im Falle einer solchen komplementären Verwendungsweise von Adverb und PP können die Tests nicht mehr als Analysekriterien dienen.

(113) Ona odevaetsja.

*Pri ètom ona $\left\{\begin{array}{l}\text { vkusnaja } \\ \begin{array}{l}\text { vedet sebja/postupaet } \\ \text { dejstvuet }\end{array}\end{array}\right\}$ vkusno. $\}$

Jedoch ist aufgrund der Analyse regelmäßiger Verwendungsweisen von Mod $_{2}$ die Klassifizierung von "so vkusom" als Mod $_{2}$ $\mathrm{zu}$ vertreten.

In einzelnen Fällen kann eine Spezialisierung der Präposition beobachtet werden, was zur Disambiguierung homonymer PP führt. So ist die PP in (115) z.B. der positive Ausdruck zu der zur Floskel erstarrten Wendung (s. (114)), die durch die doppelte Verneinung stilistisch als emotional markiert ist:

(114) Vasilij Alekseev ne bez ${ }^{39}$ boja otdaet zolotuju medal'.

(115) Oni vzjali gorod s boju.

Die nur noch in feststehenden Redewendungen als Modaladverbial gebrauchte PP (s NP ) unterscheidet sich damit auch formal von dem ambig - temporal und lokal - verwendeten Adverbial ( $v$ NP präp):

(118) On dostig svoej slavy $v$ boju. 
5.5 MODALADVERBIALE VOM TYP (VP po VP dat) MOd $_{3}$

Unter den modalen Adverbialen kann eine weitere Subklasse festgestellt werden, die als PP mit (po NP dat' gebildet wird, im folgenden $\operatorname{Mod}_{3}$. Man vgl.:

(119) On upražnjalsja po primeru roditelja.

(120) I tvorit ee sebe každyj čas po novomu proizvolu.

(121) Vse budet idti po novoj sisteme.

Bei Ubertragung der Untersuchungsergebnisse zu Mod und Mod auf diese Sätze, fällt auf, daß sich die NP der PP im Hinblick auf ihre kategorialen Merkmale von den NP der PP bei Mod und Mod $_{2}$ unterscheiden: es sind Bezeichnungen für "Dinge" und nicht für "Eigenschaften".

Dennoch enthält die PP in den Sätzen (119) - (121) eine modale Modifizierung des finiten Prädikats. Der NegationsTest (s.o. Kap. 5.1, S. $185 \mathrm{ff}$ ) verläuft mit dem die modalen Adverbiale charakterisierenden Ergebnis: es wird die Relation der in der PP gemachten Aussage zum Prädikat des Oberflächensatzes verneint, vgl.:

zu (119): On ne upražnjalsja po primeru otca. $\longleftrightarrow$ On upražnjalsja ne po primeru otca.

Es soll nun versucht werden, folgende Fragen, die sich stellen, zu beantworten:

1. Lassen sich die partiellen strukturellen Besonderheiten von Mod mit der bisher verwendeten Beschreibung modaler Adverbiale darstellen?

2. Was ist die explizite Bedeutung von $\operatorname{Mod}_{3}$ und ihr Unterschied $\mathrm{zu}$ Mod ${ }_{1}$ und $\operatorname{Mod}_{2}$ ?

Wenn wir den Prädikations-Test anwenden, erhalten wir ein Ergebnis, das, soweit das Lexikon dafür Bildungen aufweist, 
als weitere Oberflächenstruktur modaler Ausdrücke bekannt ist:

(122) On upražnjalsja tak, kak upražnjalsja roditel'.

(123) ... i tvorit ee sebe každyj cas tak, kak tvorit ee novyj proizvol.

(124) Vse budet idti tak, kak idet novaja sistema.

Mit diesem Testergebnis lassen sich die beiden gestellten Fragen insoweit beantworten, daß man sagen kann:

1. der prädikative Ausdruck des dem Adverbial zugrundegelegten Satzes wird bei der Ableitung $\mathrm{zu} \mathrm{PP}_{\mathrm{Mod}_{3}}$ getilgt. Dieser prädikative Ausdruck ist mit Präd identiscn (s. (117) - (119) und (122) - (124)) oder eine Proform dazu (s. unten (127), (128)). Die NP der PP ist - soweit überhaupt lexikalisch ausgedrückt, was z.B. nicht der Fall ist (127) (128) - das Agens des getilgten Prädikats oder eine Proform dazu.

Die Relation von Mod ${ }_{3}$ zum finiten Prädikat unterscheidet sich insofern von denen, ${ }^{3}$ die Mod und Mod ausdrücken, als Präd hier in eine Ahnlichkeits- oder Gleichheitsrelation zu einer vorher festgesetzten Art und Weise von etwas gestellt wird. In (119) ist lexikalisch diese Funktion von Mod ${ }_{3}$ durch primer ausgedrückt. Ein Synonym dafür ist obrazec:

(125) Sostavljajte predloženija po obrazcu: ...

Eine weitere Oberflächenstruktur dieser Modaladverbialien ist soglasno NP dat' die z.B. bei einer Aufzählung neben der PP po NP auftreten kann:

(126) Ob ètom $v$ Castnosti govorit bol'saja raznica ¿islennosti zanjatych - soglasno perepisi i po tekušcemu šcetu. 
$\operatorname{Mod}_{3}$ kann die elliptische Form (po Adat) haben:

(127) Ja ... nacal slovno po-pisanomu.

(128) Vse vydadi, pokupki po-prežnemu ot nee zaviseli.

(129) Vse ostalos' po-staromu.

Getilgt sind dabei Lexeme mit der Semantik "vid", "sposob". Eine weitere, heute als Adverb grammatikalisierte Form von $\operatorname{Mod}_{3}$ liegt vor in:

(130) Ivan Petrovic ... odel syna po-šotlandski.

die inzwischen Adverb für Nationalitätenbezeichnungen schlechthin geworden ist.

Die PP (po NP ) ist in mannigfaltiger Beziehung homonym (vgl. o. Kap. 4at 4.2, S. $172 \mathrm{ff}$ ). Für die Auflösung der damit möglichen Ambiguität sind der Prädikations-Test und der Negations-Test dienlich, wie oben (S. $189 \mathrm{f}$ ) gezeigt werden konnte.

Zu den Möglichkeiten von Homonymie bei (po NP dat): nämlich als Ausdruck temporaler, modaler und kausaler Adverbiale verwendet werden $z u$ können, kommt noch eine weitere hinzu, auf die Beispiele vom Typ (126) aufmerksam machen. Man vgl.:

(131) On byl nastojašłij mužik, po vyraženiju Glafiry Petrovny.

(132) Vot po ich-to podščetu i polucilos', cto ...

(133) Po našemu (mneniju), on vinovat.

(134) Po verojatnosti, on byl nastojašij mužik.

Versucht man mit Hilfe des Prädikations-Tests die Relation $z w i s c h e n$ Adverbial und den übrigen Satzgliedern zu bestimmen, so erhält man für (131): 
On byl nastojašxij mužik, tak $\left\{\begin{array}{l}\text { vyražaetsja } \\ \text { vyražalas' }\end{array}\right\}$

- Tak - stellt hier nicht eine Ähnlichkeitsrelation zu Präd auf, sondern zu einem getilgten Verb aus der Klasse der performativen Verben, von dem strukturell die Aussage, "daß ..." abhängt. Diese tiefenstrukturellen Verhältnisse, auf die ich hier nur verweisen.kann, finden oben in (132) ihren Ausdruck: Die Aussage, "daß ..." wird hier auch formal durch das Lexem polucilos' eingeleitet, und zu diesem drückt die PP po ich-to podకčtu eine Ähnlichkeitsrelation aus. Die verbreitete Tilgung des performativen Ausdrucks kann wohl in Zusammenhang damit gesehen werden, daß die PP (po NP dat) in (133) und (134) als Satzadverbial ${ }^{42}$ verstanden wird. Es ist diese weitere Funktion, die bei der Ambiguität von (po NP dat) in Rechnung gestellt werden muß. Man vgl. zu (134) die synonyme Form:

(135) Verojatno, ¿to on byl nastojašij mužik. 


\section{EXKURSE}

\subsection{EXKURS 1}

Unter den Modaladverbialen fällt eine Gruppe von PP (s NP instr' auf, die bei der Anwendung der Tests gewisse Unterschiede $z u$ Mod 2 aufweist, obwohl andererseits eine Beziehung zu dieser Adverbialklasse offensichtlich ist. Ich meine PP vom Typ "s trudom", bei denen es sich um eine lexikalisch relativ kleine Gruppe ${ }^{43}$ handelt; häufig vertreten sind "s trudom": "s legkost'ju" und "s pol'zoj", z.B.:

(1) On uchodil ot takogo napadenija s trudom.

(2) On rešal trudnejšie zadači s legkost'ju.

(3) On provel studenčeskie gody za rubežom s pol'zoj.

Bei der Anwendung des Prädikations-Tests erhalten wir eine struktur, die auch als Oberflächenstruktur vertreten sein kann:

zu (1) Kak on uchodil ot takogo napadenija, bylo trudno $\left\{\begin{array}{l}\text { dlja nego. } \\ \text { emu. }\end{array}\right\}$

zu (2) Kak on resal trudnejšie zadači, bylo legko $\left\{\begin{array}{l}\text { dlja nego. } \\ \text { emu. }\end{array}\right\}$

Aus dem Prädikations-Test läßt sich erkennen, daß die PP Präd modal näher bestimmt, indem sie eine Aussage über das "wie" der Handlung macht. Insoweit ist die Funktion der PP der von Mod ähnlich. Allerdings werden die Unterschiede ebenfalls deutlich: man gerät in Schwierigkeiten, wenn man nach den Regeln für Mod und $\operatorname{Mod}_{2}{ }^{\prime}$ die aus den Prädikatsvariablen-Tests gewonnen wurden, hier versucht, das "wie" der Modifizierung näher zu bestimmen. Mir scheint das damit zusammenzuhängen, daß die Prädikate, auf die ich die PP in (1-3) zurückführe - trudno, legko, polezno - sich in ihren satzsemantischen Funktionen von denen, die PP Mod zugrunde- 
gelegt wurden, unterscheiden. So ist mit diesen Prädikaten kein "Agens" prädizierbar, sie schreiben vielmehr einem " $X$ " (hier ausgedrückt durch Präd) einen Zustand zu. Daher wäre wohl eine Beschreibung nicht adäquat, die $z u$ dem Schluß kommt, daß mit, den PP in (1-3) das "wie" des "Handlungsvollzugs beschrieben wird, was, wie 0 . bei Mod gezeigt wurde, eine Aussage über den, der die Handlung vollzieht, impliziert.

Im Zusammenhang mit den möglichen satzsemantischen Funktionen dieser Klasse prädikativer Ausdrücke ist eine weitere Besonderheit der Sätze vom Typ (1-3) zu sehen: (3) kann auch bedeuten:

(4) Cto on provel studenCeskie gody za rubežom, bylo polezno dija nego.

Das heibt, die PP dieser Klasse können als Satzadverbial verwendet werden, und dieses macht bekanntlich eine Aussage über die "Tatsache, daß ..."

Die beschriebene Ambiguität ist bei einer Reihe von Sätzen mit (s NP ${ }_{\text {instr }}$ ) dieser Klasse zu beobachten:

(5) On rasstavalsja s prošlym s trudom.

Kak on rasstavalsja s proslym, bylo trudno emu. Cto on rasstavalsja s prošlym, bylo trudno emu.

Entsprechendes gilt auch für den Satz nach der Infinitiv-Tf:

(6) Rasstat'sja s proslym bylo trudno emu.

Als abschließendes Beispiel für die hier beschriebene Ambiguität seinen genannt:

(7) Ona ne možet rasprostranit' ich s osobennoj polzoj imeja malo intelligentnogo znakomstva.

(8) ... oni imejut vozmoznost' posil'no rabotat's pol'zoj dlja sem'i. 
6.2 EXKURS 2: PP (bez $\mathrm{NP}_{\text {gen }}$ )

Anders als bei den lokalen und temporalen Adverbialen gibt es für die modalen Adverbiale zum Ausdruck der Verneinung eine PP, und zwar (bez NP ). Diese PP wird hier nur als Exkurs behandelt, weil sie in mehrfacher Hinsicht mehrdeutig ist und die Beschreibung der daraus resultierenden homonymen Strukturen weitere Untersuchungen $z u$ anderen Klassen von Adverbialen notwendig macht. In der Verneinung, ausgedrückt mit (bez NP,' ist formal der Unterschied der bisher behandelten Modaladverbialklassen neutralisiert. Das verdeutlichen im besonderen Minimalpaare (s. die folgenden Beispiele), deren Bildung möglich ist, insoweit die logisch-semantischen Verhältnisse sinnvolle Sätze ergeben, und zwar sowohl bei Affirmation als auch bei Negation der Aussage über identische Argumente. (Für die Demonstration erwiesen sich gewisse Ersetzungen der lexikalischen Elemente als notwendig; sie berühren jedoch nicht die Struktur.) Man vgl.:

zu $\operatorname{Mod}_{1}:$

(1) Oni žili v gore. - Oni žili bez gorja.

(2) Noči prochodjat $v$ neistoščimom veselii i ščastii. - Noči prochodjat bez veselija i ščastija.

(3) Oni sideli $v$ molčanii. - Oni sideli bez dviženija.

(4) Obraz lizy vosstal vo vsej svoej jasnosti. - Obraz Lizy vosstal bez obyčnoj jasnosti.

(5) Otčety byli postavleny na rassmotrenie $v$ objazatel'nom porjadke.

- otčety byli postavleny bez vsjakogo porjadka.

zu $\operatorname{Mod}_{2}:$

(6) ... proiznesla Liza s zapinkoj. - ... proiznesla Liza bez zapinki. 
(7) ... sprosil on s volneniem. - sprosil on bez volnenija.

(8) ... on s rezkost'ju vedet svoi ataki - On bez rezkosti vedet svoi ataki.

(9) Ona s usiliem pojmala ruku muza. - Ona bez usilija pojmala ruku muža.

(10) Ona s uspechom razvodila pautinu. - Ona bez uspecha razvodila pautinu.

(11) On s trudom uchodil ot takogo napadenija. - On bez truda uchodil ot takogo napadenija.

(12) On s pol'zoj provel studenðeskie gody. - On bez pol'zy provel studen eskie gody.

Die Generalisierung der Form (bez NP )' im Verhältnis zu ihren Funktionen als modale Adverbiale hat zur Folge, daB grammatische Homonyme entstehen:

(13) Ona tol'ko posmotrela na menja bez udivlenija i pošla proc'.

ist antonym zu:

- Ona tol'ko posmotrela na menja $v$ udivlenii i pošla proč. und

- Ona tol'ko posmotrela na menja s udivleniem i pošla proč'.

Abgesehen von dieser Generalisierung bei der Verwendung von (bez NP, innerhalb der Klasse der modalen Adverbiale ist diese $\mathrm{PP}$ aufgrund ihrer möglichen zuordnung $z \mathrm{u}$ anderen Klassen fakultativer Adverbiale homonym, z.B. zu der kooperationalen (deren bejahte Form bekanntlich auch die PP (s NP instr' ist).

Man vgl.:

(14) Ljubov' sošla $v$ ego grud' bez radosti $i$ bez mučenij. Ljubov' sošla v ego grud' so vseju neistošximoju radost'ju i so vsemi tomitel'nymi mučenijami. 
Die Testprobe mit dem Prädikatsvariablen-Test für $\operatorname{Mod}_{2}$ ergibt:

[Kak l jubov' sošla v ego grud',] proisošlo radostno $i$ mučcitel 'no.

Als vorläufigen Test für kooperationale Adverbiale schlage ich vor:

Ljubov' sošla v ego grud'. Sošli v ego grud' radost' i muČenija.

Die PP ist modales oder kooperationales Adverbial ( (a) und b)) oder Attribut zum Objekt (c):

(15) Širitsja sorevnovanie za provedenie senokosa bez poter'. ... provodjat senokos s poterjami. (a)

... provodjat bez poter' senokos.

... provodjat senokos bez poter'.

Folgende Beispiele sollen weitere Verflechtungen der Adverbialklassen, ausgedrückt durch (bez NP gen ) demonstrieren:

(16) Novaja konstitucija garantiruet osnovnye prava bez različj rasovoj prinadležnosti.

(17) Važno naladit' vzaimodejstvie vsech uXastnikov processa bez iskljuðenija smežnikov.

(18) Uvelicitsja proizvodstvo metalla bez vvoda novych mošrnostej.

(19) Na nem možno transportirovat' bez peregruzok truby.

(20) Bez uČeta mnenija kommunistov stranoj upravljat' nevozmoźno.

(21) Nevozmožno obespę̌t' podlinnuju demokratiju bez uXastija kommunistov vo vsech sferach. 
Der Versuch, mit Hilfe der hier verwendeten Tests die satzsemantischen Relationen der PP zu Präd als modal oder kooperational zu explizieren, bleibt unbefriedigend. Die Voraussetzungen des damit angesprochenen Problems machen (29) und (21) deutlich: ist Präd negiert, gibt die Aussage in der Form PP (bez NP gen) die "Bedingung" für Präd an. Diese Zusammenhänge zwischen modalem, kooperationalem und konditionalem Adverbial. - auf die hier nur hingewiesen sei werden deutlich, wenn wir anstelle des negierten Prädikats das bejahte verwenden:

(22) Upravljajut stranoj bez uCeta mnenija kommunistov.

(23) Vozmozno obespecit' podlinnuju demokratiju bez ucastija kommunistov.

Man kann leicht erkennen, daß es für die PP (bez NP,) in (16-21) eine weitere Oberflächenstruktur gibt, das gendverbialpartizip, dessen Besonderheit - nämlich seine funktionale Mehrdeutigkeit - immer wieder zu linguistischen Analysen herausfordert (Rủzicka, 1980, Kap. V und VI).

Man vgl.:

(24) Novaja konstitucija garantiruet osnovnye prava, ne različaja rasovoj prinadležnosti.

(25) Važno naladit' vzaimodejstvie vsech učastnikov processa, ne isključaja smežnikov.

Entsprechend läßt sich zu (18) und (19) bilden:

nach Rückführung der Nominalisierungs-Tf :

(26) Ne vvodja novych mošnostej proizvodjat metall.

nach Rekonstruktion des getilgten obligatorischen Aktanten:

(27) Na nem možno transportirovat' truby, ne peregružaja ego. 


\section{ZUSAMMENFASSUNG}

Geht man von der Definition des sprachlichen zeichens als Summe seiner distinktiven Merkmale aus, so ist zu der Klasse der freien Adverbiale $z u$ sagen, daß sie als Glied des Satzes und als Klasse von syntaktischen Einheiten schwach determiniert ist. Die vorliegende Arbeit ist der Untersuchung der Adverbiale in der Form der Präpositionalphrase gewidmet, beschränkt auf die drei Klassen: lokal, temporal, modal. In der Form der PP ist das Untersuchungsobjekt in größerer Vielfalt vertreten als in der Wortart Adverb, was nicht heiBt, ${ }_{45} a$ damit auch alle verwendungsmöglichkeiten der Adverbien erfaßt sind. Im Russischen können Träger der distinktiven Merkmale des Adverbials als PP nur sein: Präposition, Nomen der PP, Wortstellung und Intonation (letztere wurde in dieser Arbeit nicht eigens experimentell untersucht).

Alle drei hier behandelten Elemente sind bekanntlich funktional mehrdeutig: für die Präposition erinnern wir nur daran, daß ein- und dasselbe zeichen z.B. im Rahmen der Rektion als syntaktisches Mittel fungiert, dagegen, verwendet zum Ausdruck von Adverbialen, primär als semantisches.

Die Mehrdeutigkeit des Nomens der PP hängt von seiner lexikalischen Besetzung $a b$ : bei semantisch relativ einfachen Bezeichnungen z.B. für ortsangaben (Kap. 3.1, S. $61 \mathrm{ff}$ ) ist die zuordnung $z u$ der Subklasse der Lokaladverbiale in der Regel eindeutig; dagegen ist die Relation eines solchen Adverbials $z u$ den übrigen Satzgliedern nicht selten mehrdeutig (Kap. 3.2, S. $86 \mathrm{ff}$ ). Anders sind die Verhältnisse bei den Temporal- und Modaladverbialen: vereinfacht gesagt, kann man hier feststellen, daß die Beziehung $z u$ den übrigen Satzgliedern in der Regel eindeutig ist (s. z.B. Kap. 4.1.1, S. $166 \mathrm{ff})$, dagegen ist - motiviert noch durch die Mehrdeutigkeit der Präposition - die Zuordnung des Adverbials $z u$ einer seiner Subklassen oft nicht ohne Rückgriff auf 
Kontext oder Vorwissen möglich (s. z.B. Kap. 4.4.2, S. $172 \mathrm{ff}$ und Exkurs 2, S. 223 ff).

Die Wortstellung als Ausdrucksmittel der aktuellen Satzperspektive kann für die Beziehung des Adverbials zu den übrigen Satzgliedern relevant sein - durch sie können Mehrdeutigkeiten eingegrenzt sein, jedoch kann sie diese auch geradezu implizieren. Von besonderer Bedeutung ist die Grenze von Thema und Rhema ( $s$. Kap. 3.2, besonders S. $100 \mathrm{ff}$ ).

Bei einer so schwachen formalen Determiniertheit der freien Adverbialen als PP ist es naheliegend, daß die neuere syntaxforschung, die sich gerade an den Formen orientiert, diese freien Adverbiale zwar als problem nennt, sie aber in ihrer Grammatikkonzeption nicht adäquat berücksichtigt (die vorliegende Arbeit stützt sich mit Einschränkungen auf das Standardmodell, aber auch die Version der "Extended Standard Theory" enthält in dieser Hinsicht keine essentiellen Verbesserungen).

Andererseits muß auch die vage Bestimmung der freien Adverbiale als "Determinanty" in deskriptiven Grammatiken (z.B. in der sowjetischen Russistik, s. S. 17 und 180) im zusammenhang mit der Mehrfunktionalität, bedingt durch die schwache formale Determiniertheit, gesehen werden. Die Abhandlung der Beispiele erfolgt in Listen, die Aufzählungen gleichen und deren ordnungskriterien widersprüchlich sind.

Die Konzeption, auf die die vorliegende Untersuchung sich gründet, wurde in Kap. 2, besonders $2.2 \mathrm{~s}$. $51 \mathrm{ff}$, dargelegt. Hier sollen die allgemeinen Ergebnisse, die aus den Analysen der Lokaladverbiale (Kap. 3), der Temporaladverbiale (Kap. 4) und der Modaladverbiale (Kap. 5) gewonnen wurden, zusammengefaßt werden.

1. Die freien Adverbialen sind eine Oberflächenform zum Ausdruck zugrundeliegender Prädikationen unterschiedlicher Klassen. Die für die Untersuchung der Adverbiale inter- 
essante TS ist die Ebene, die die Beziehungen des tiefenstrukturellen Prädikats zu seinen Argumenten explizit macht. Mit der Wahl dieser TS stehen satzsemantische Untersuchungen im Mittelpunkt dieser Arbeit. Die Untersuchungen der Beziehungen der Satzelemente unter dem genannten Aspekt brachten Aufschlüsse zu folgenden Fragenkomplexen:

- Beziehung des Adverbials zu den übrigen Satzgliedern;

- Erweiterungs- und Blockierungsmöglichkeiten von Oberflächenstrukturen mit den einzelnen subklassen der freien Adverbiale (für die Erweiterung mit den hier untersuchten Adverbialen allgemein: S. $206 \mathrm{f}$; mit Advb : S. $101 \mathrm{f}$; mit ${ }_{\text {temp }}^{\text {Advb }}$ S. $137 \mathrm{ff}$; mit Advb $_{\text {mod }}$ : S. $189,198 \mathrm{loc}$ f, $\left.209 \mathrm{f}\right)$;

- systematische syntaktische Relationen der Adverbiale zu anderen Oberflächenstrukturen.

Bei den Untersuchungen wurden u.a. im Sinne von heuristischen Mitteln bestimmte Klassen von Prädikatsvariablen herangezogen, die prädikatenlogischen Analysen entnommen wurden. Ich bin mir darüber im klaren, daß bei den Untersuchungsergebnissen Fragen offen bleiben, die zum einen durch die generellere Ebene der Prädikatenlogik, zum anderen durch die speziellere, z.B. der Wortbildung, bedingt sind.

2. Die freien Adverbiale haben verschiedene und unterschiedliche Relationen $z u$ den übrigen Satzgliedern, die, wenn auch durch die Formen ( $s$. hier vor allem die Wortfolge) nur schwach materialisiert, so doch im Oberflächensatz durch die Fähigkeit, Kommunikation zu gewährleisten, nachprüfbar sind. Insofern muß man feststellen, daß die freien Adverbiale syntaktische Funktionen ausdrücken. Verschieden sind ihre Relationen zu den übrigen Satzgliedern insoweit, als sie sich auf verschiedene Klassen von Satzgliedern beziehen können: die Lokaladverbiale auf NP und VP, die Temporal- und 
Modaladverbialen auf VP (vgl. S. $125 \mathrm{f} ; 141 \mathrm{f}, 198 \mathrm{f}$ ). Die Unterschiede in den Relationen liegen darin, daß eine Menge freier Adverbiale $z u$ den koordinierenden sprachlichen Mitteln gehört (s. Kap. "Lokaladverbiale", besonders S. 70, $73 \mathrm{ff}$ ), eine andere Menge $z u$ den subordinierenden (s. Kap. "Temporaladverbiale", besonders S. $141 \mathrm{f}, 154)^{46}$.

3. In den traditionellen Grammatiken 2.B. werden die Adverbiale häufig in dem Kap. "Primykanie" ("Adjunktion") abgehandelt. Versucht man, die Ergebnisse der vorliegenden Arbeit in diese grammatischen Traditionen einzuordnen, kann man sagen, daß auch vom generativ-transformationellen Standpunkt aus die Unterscheidung der Typen syntaktischer Abhängigkeiten in Rektion und Adjunktion ihre prinzipielle Bestätigung findet. Darüberhinaus meine ich feststellen zu können, daß die Ergebnisse zu den syntaktischen Funktionen der Adverbiale, die mit dem hier angewendeten Beschreibungsansatz festgestellt wurden, einen Beitrag $\mathrm{zu}$ einem besseren Verständnis der formalen Besonderheiten der Adjunktion, vertreten durch die freien Adverbiale, im Unterschied zu denen der Rektion liefern.

Die Versuche, den freien Adverbialen einen Platz in der TS von Modellen wie der "Standardtheorie" zuzuweisen, mußten scheitern, da die dabei vorgesehenen Relationen prinzipiell von dem Typ " $y$ hängt ab von $x^{\prime \prime}$ sind: die Elemente der Ersetzungsregeln sind Glieder, die in dieser Relation zueinander stehen; bekanntlich erscheinen sie als Vorstufe des' Oberflächensatzes in der Terminalkette.

Nach diesem Expandierungsschema für \#S\# läßt sich die Adjunktion nicht ableiten, weil sie zu keinem anderen Satzglied in der Relation "hängt ab von" steht. Ich möchte deutlich machen, das die spezifischen Funktionen der freien Adverbiale vielmehr mit Hilfe von Satzverknüpfungsmodellen nachgewiesen werden müssen. Dabei erbrachten die Untersuchungen, daß relativ oberflächennah ein Satz mit freiem Adverbial mehr als e i n e Proposition beinhaltet. Eine 
andere Oberflächenstruktur wäre 2.B., jede dieser Propositionen als Satz zu erzeugen. Nehmen wir den Gedanken von der Gegenüberstellung: Rektion gegenüber Adjunktion wieder auf, so führt uns die tiefenstrukturelle Analyse $z u$ dem Schluß, daß die Adjunktion in ihrer Relation $z u$ den übrigen Satzgliedern sich dadurch auszeichnet, daß sie sich gegenseitig nicht bedingen ${ }^{48}$.

4. Die strukturellen Besonderheiten der Adverbiale wurde mit Hilfe operationeller Tests untersucht. Neben der Aufdeckung vielfacher Homonymien, die bei den genannten formalen Gegebenheiten $z \mathrm{u}$ erwarten sind, konnte - m.E. präziser als bisher - nachgewiesen werden, inwieweit die freien Adverbiale in syntaktischen Relationen $z u$ anderen Oberflächenstrukturen stehen. Diese syntaktischen Beziehungen stehen mit den vielseitigen Funktionen in Zusammenhang, die oben Punkt 2 zusammengefaßt wurden, was man vielleicht für ein weiteres Indiz für die verzweigten Interrelationen sprachlicher Strukturen deuten kann.

Der Nachweis, daß es sich bei diesen Beziehungen um im weiteren sinn syntaktische synonymien handelt (nicht Synonymie in sensu stricto, s. die Ausführungen oben S. 38 f), wurde mit Hilfe eines tiefenstrukturellen Modells geführt. Dieses setzt syntaktische und semantische strukturen zueinander in Beziehung, ordnet diese, indem es sie auf Hierarchieentsprechung und Hierarchiedivergenz überprüft, einander zu. In diesem Sinn stehen in systematischen syntaktischen Relationen:

$$
\begin{aligned}
& \text { PP loc und } S \text { (vgl. S. } 58 \mathrm{ff}, 68 \text { ) } \\
& \text { "loc "NS }(\mathrm{vgl} \text {. S. } 73 \mathrm{ff})
\end{aligned}
$$

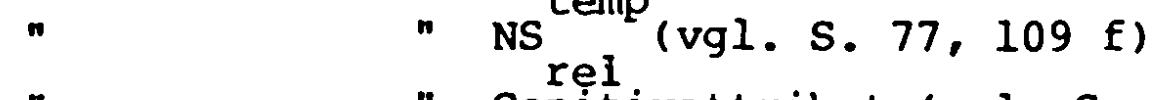

$$
\begin{aligned}
& \text { " Genitivattribut (vgl. S. 109) } \\
& \left."\left(\text { bei VP }{ }_{\text {pass }}\right) " N P^{l(A d v b)} \text { (bei VP }{ }_{\text {akt }} \text { ) (vgl. S. } 84 \mathrm{f}\right)
\end{aligned}
$$




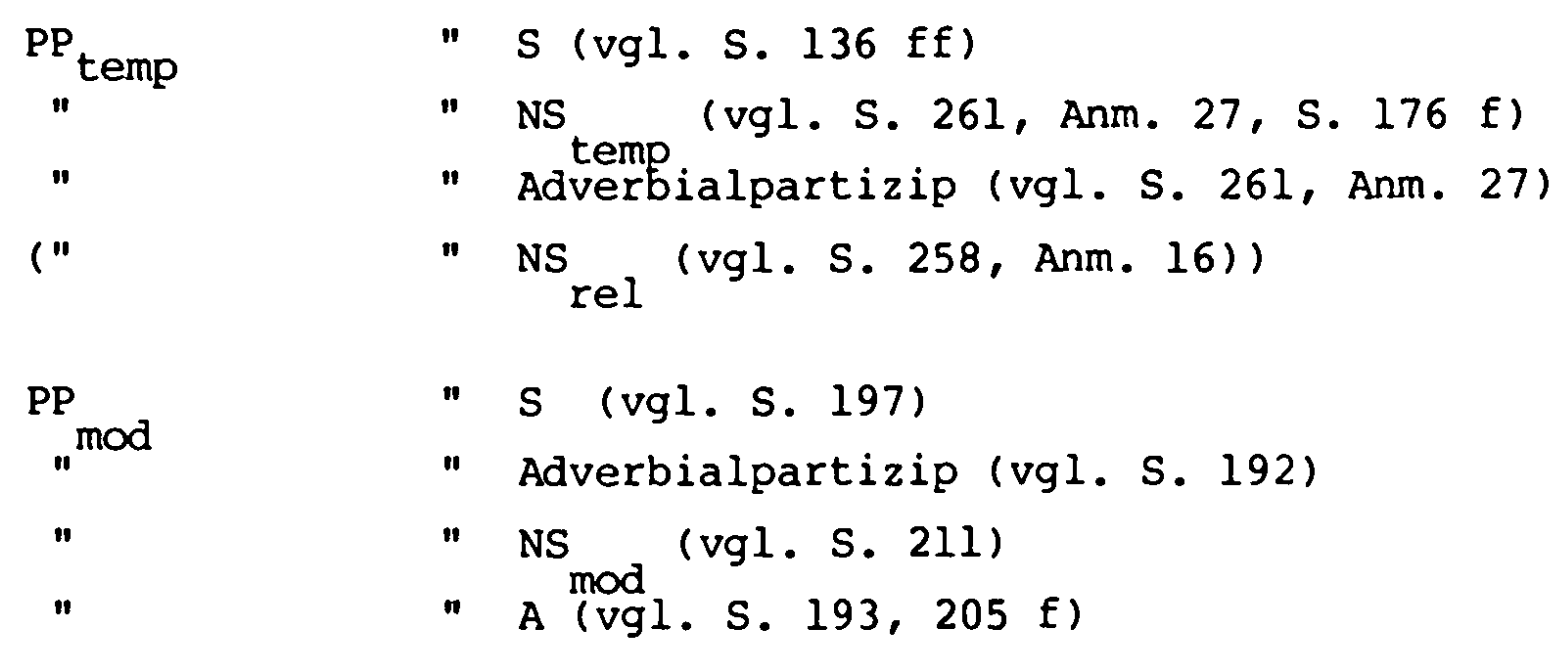

Eine komplexere Derivationsgeschichte als bei diesen Strukturen liegt bei den folgenden vor:

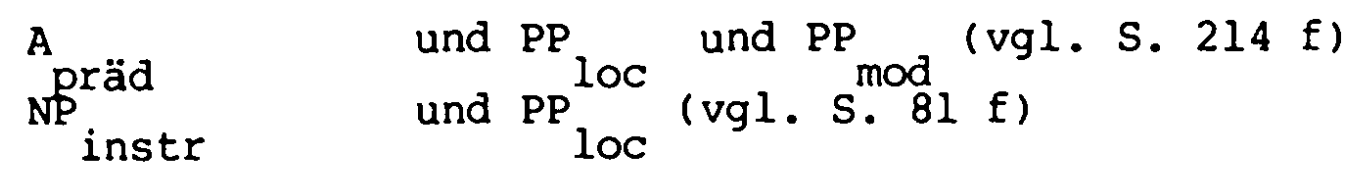

5. Die Ergebnisse machen auf methodologische Implikationen des hier gewählten Beschreibungsmodells aufmerksam. Ich sehe in satzsemantischen Untersuchungen eine fruchtbare Fragestellung zur Lösung auch wortsemantischer Probleme (vgl. S. 206 f); andererseits sind methodologische Grenzen deutlich geworden, die sich bei der Gegenüberstellung des tiefenstrukturellen Konstrukts mit den Oberflächensätzen ergaben: s. für $P P_{l o c}$ in Anfangsstellung (S. 124 f); für die Aspektwahl von $V P$ in Sätzen mit Advb Temp ${ }_{10 C}$ (S. 164 f); für die Unterscheidung der adverbialen Subklassen temporal, konditional, kausal, modal (S. 175 f, 178 f). 


\section{ANHANG \\ 8.1 ANMERKUNGEN}

ANMERKUNGEN ZU KAPITEL 1:

1 - Bartsch, R., Adverbialsemantik, Frankfurt, 1972, bes. Kap. IXX; Koktová, E., Are Local Adverbials Derived from Temporal Sources? in: P Bulletin ML, 27, 1977, 45-63.

2 - wichtige Voruntersuchungen zur Bestimmung des "Verbrahmens" sind in dem Publikationsorgan P Bulletin ML der Prager Karlsuniversität erschienen, z.B. Panevová, J., On Verbal Frames in Functional Generative Description, part I, P Bulletin ML, 22, 1974, 3-40; diess., part. II, loc. cit., 23, 1975, 17-52; diess., Verbal Frames Revisited, loc. cit. 28, 1977, 55-71; diess., Fragen der Nominalisierung zeitlicher Prädikation, loc. cit., 30, 1978a, 9-42; diess., Inner Participants and Free Adverbials, in: P Studies ML, 6, 1978b, 227-254 .

Demgegenüber kommt die Arbeit von Kučera, H./Trnka, K., Time In Language (Temporal Adverbial Constructions in Czech, Russian and English), Michigan, 1975, über die methodischen Schwierigkeiten, auf die bereits Steinitz aufmerksam macht ausfuhrlicher dazu u. - nicht hinaus.

Auch auf dem Boden eines anderen Beschreibungsansatzes der neueren Syntaxlehre, nämlich der Kasusgrammatik, werden konsequenterweise - die freien Adverbialen nicht behandelt; vgl. Freidhof, G., Kasusgrammatik und lokaler Ausdruck im Russischen, München, 1978. Das Interesse, das in Fachkreisen seit je dem Problem der Adverbialen entgegengebracht wurde, dokumentiert mit seiner Bibliographie L. Siegrist (1976), zum (leider fast ausschlieblich) deutschen und englischen Adverbial. 
3 - Chomsky, N., Aspects of the Theorie of Syntax, 1965; es wird nach der Ausgabe "Aspekte der Syntax - Theorie", Frankfurt, 1970 (im ff Standardmodell) zitiert.

Die folgenden kritischen Auseinandersetzungen mit der syntaktischen Beschreibungen der Adverbiale bei Chomsky beziehen sich im wesentlichen auf das Standardmodell. Ich habe mich für diese Konzeption der Chomsky'schen Syntaxtheorie entschieden, weil die "Extended Standard Theory" (Chomsky 1971) zu den Adverbialen keine weiterführenden Vorschläge enthält.

4 - Vgl. Coseriu, E., Leistung und Grenzen der transformationellen Grammatik, Tübingen 1977, $45 \mathrm{ff}$; symptomatisch sind dafür auch die Publikationen von F. Kiefer, z.B. "Studies in Syntax and Semantics", Dordrecht, 1969; "Semantik und generative Grammatik", I, II, Dordrecht, 1972.

5 - Vgl. Jacobs, R., Rosenbaum, P. (Hrsg.), English Transformational Grammar, Waltham Mass., 1968, im besonderen chapt. 27, Manner Adverbials, 228-231.

6 - Die Abkürzung "Advb" für freie Adverbiale im Unterschied zu "Adv" für obligatorische Adverbiale übernehme ich von Steinitz (1971).

7 - Ich beschränke mich hier auf die im westen allgemein zugänglichen Titel: Sgall, P., Hajił̌vá, E., BeneSová, E., Topic, Focus and Generative Semantics, Kronberg, 1973. Funcional Generative Grammar in Prague (Prager Autorengruppe), Kronberg, 1973. Kürzlich erschien als überarbeitete Fassung der FGB: Contributions To Functional Syntax And Semantics (1985), hrsg. V. P. Sgall, Philadelphia, Amsterdam.

8 - Sgall, P., K metodam opisanija sintaksisa, in: P Bulletin ML, 27, 1977, 5-20. 
9 - Rưzicka, R., Studien zum Verhältnis von Syntax und Semantik im modernen Russisch, Berlin, 1980; Zimmermann, I., Sintaksiceskie funkcii aktantov, zalog i perechodnost', in: Chrakouskij, V., Hrsg., Problemy teorii grammatičeskogo zaloga, Moskva, 1978, 71-79.

10 - Krylova, O.A., Determinanty $v$ aspekte kommunikativnogo sintaksisa, in: VJa, 1976, 2, 43-52, bes. 43; vgl. auch die Literaturangaben dort.

11 - Šedova, N., Ju., Determinirujušxij ob'ekt i determinirujuscee obstojatel'stvo kak samostojatel'nye rasprostraniteli predloženija, in: VJa (1964), 6, 77-93. Die neue Akademiegrammatik (1982) steht ebenfalls in dieser Tradition. Sie behandelt die freien Adverbiale in dem Kapitel über "Rasprostranenie predloženija v celom (determinacija)" vgl. Russkaja grammatika, 1982, 2, 149 ff. Es kommen dabei Beobachtungen über die Beziehungen zwischen den "adverbialen Determinanten" (obstojatel'stvennye determinanty) zum übrigen Satz, im besonderen zum prädikat, zur Sprache, die man durchaus als intuitiv zutreffend empfunden bezeichnen kann; jedoch werden daraus keine Schlüsse für eine Analysemethode abgeleitet.' Man vgl. zur Definition allgemein: "Die Ergänzung, die sich auf den ganzen Bestand des Satzes bezieht und nicht mit einem seiner Einzelglieder verknüpft ist, heißt

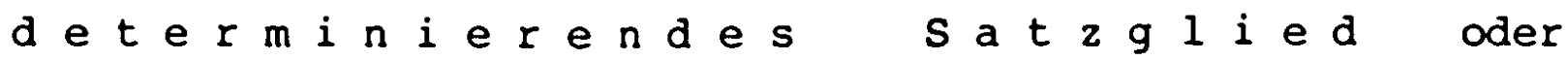
Determ $\mathrm{i} n$ a $n t$.... Der Determinant ist keinem bestimnten Satzglied untergeordnet, aber das heiBt nicht, daß eine Verknüpfung mit dem Satz im ganzen überhaupt fehlt: er ist durch einen losen Anschluß mit dem Satz verknüpft, ..." (149). Zu den "adverbialen Determinanten", auch "determinierende Adverbiale" genannt, im.. besonderen heibt es: "In den Sätzen mit einem Verb kann man immer eine unmittelbar unterordnende Beziehung zwischen diesem Verb und dem adverbialen Determinanten als möglich wahrnehmen. Die 
Beziehung, die aus der Verknüpfung des Determinanten mit diesem verb entsteht, is $t \quad e i n e s$ e $m$ a $t$ i s $c h$ n i cht vor hersag bare Bezi e hu n g, was ein Merkmal für die Unabhängigkeit des Determinanten vom Verb in diesen Fällen ist. So ist z.B. in dem Satz 'Tolstoj v dnevnike oxen' volnovalsja iz-za ètogo pis'ma (Skl)' die Beziehung 'volnovalsja $v$ dnevnike' grammatisch richtig, aber semantisch zufällig (nicht systembedingt): sie ist nicht nur aufgrund der lexikalischen Bedeutungen der wörter nicht vorhersagbar, sondern sie ist auch offensichtlich mit diesen Bedeutungen nicht verknüpft. ... In dergleichen Fällen haben wir keine unterordnende Beziehung der wörter vor uns, sondern eine adverbiale Determination des Satzes durch ein obliques Nomen" ( 159 f). Danach folgt eine umfangreiche Beispielliste.

12 - Das gilt selbstverständlich "cum grano salis"; umso mehr, als man in Rechnung stellen muß, daß die Fachliteratur u.U. schwer oder nicht zugänglich ist. $\mathrm{Zu}$ den Ausnahmen zählen z.B. die Arbeiten von zolotova, G.A., Oxerki funkcional'nogo sintaksisa russkogo jazyka, Moskva, 1973; diess., Kommunikativnye aspekty russkogo sintaksisa, Moskva, 1982 .

13 - Bei dieser Forschungslage in der Russistik ist es m.E. nach wie vor berechtigt, für die Beschreibung russischer Adverbiale die vor ca. 15 Jahren geschriebene Arbeit von D.B. Johnson (Transformations And Their Use In The Resolution of Syntactic Homomorphy. Prepositional or constructions in Contemporary Standard Russian, The Hague, 1970) auszuwerten, in der mit Hilfe von Transformationen Harris'scher Prägung methodisch gesicherte Klassen von Präpositionalphrasen (PP) des Russischen aufgestellt wurden. 
ANMERKUNGEN ZU KAPITEL 2:

1 - zit. aus Stein, w. (Hrsg.), Bismarck; Des eisernen Kanzlers Leben in annähernd 200 Bildern nebst einer Einführung, Siegen/Leipzig, 1915, 17.

2 - Nach Jakobson, R., Beitrag zur allgemeinen Kasuslehre. Gesamtbedeutungen der russischen Kasus, 1935. Zit. nach $R$. Jakobson, Form und Sinn, 98 f, München 1974 .

3 - auf mögliche unterschiedliche Funktionen der Adverbien und der PP als Adverbiale macht Pitha mit folgendem Beispielpaar aus dem Tschechischen aufmerksam: "vlevo nahoře" ändert die Bedeutung bei Veränderung der Wortfolge nicht: "nahoře vlevo". Dagegen kann das der Fall sein bei PP: "na stole vlevo" - "vlevo na stole". Pitha, P., Nekotorye zameđanija $k$ obrabotke morfologii obstojatel'stva mesta $v$ generatiunom opisanii Zešskogo jazyka s neskol'kimi urovnjami, in: $\mathrm{p}$ studies ML, 3, 1972, 241-257, bes. 250.

4 - als neuere Darstellung verweise ich auf: Grammatika (Praha) 1979, Bd. 2, $752 \mathrm{ff}$.

5 - s. Grammatika (1970), die umfangreiche Beispielsammlung zu "Imennoe primykanie" (516-535).

6 - Schüller, M., N P N-Konstruktionen im Rusșischen. Versuch einer grammatisch-semantischen Analyse von $\mathrm{N}^{1}$ po $\mathrm{N}^{2}$, masch.geschr., Saarbrücken, 1976 (Dipl.-Arbeit).

Trenczek, H., N P N-Konstruktionen im Russischen. Versuch einer grammatisch-semantischen Analyse von $\mathrm{N}^{1}$ za $\mathrm{N}^{2}, \mathrm{~N}^{1} \mathrm{~s}^{2}$, masch.-geschr., Saarbrücken, 1976 (Dipl.-Arbeit).

Hinzer, R., N P N-Konstruktionen im Russischen. Versuch einer grammatisch-semantischen Analyse von $\mathrm{N}^{1} \circ \mathrm{N}^{2}$, masch.-geschr., Saarbrücken, 1978 (Dipl.-Arbeit). 
7 - Vorschläge für Unterscheidungskriterien für Präpositionalobjekte einerseits und Adverbiale andererseits legt Zimmermann, I. vor; vgl. diess., Die substantivische Satzform, in: zeitschrift für slawistik, 17, 1972, 674-684.

8 - Im Sinne der von Chomsky hypothetisch festgelegten Beziehung zwischen Syntax und Semantik werden mit "'Paraphrase eines Satzes'" Sätze bezeichnet, "die nicht nur die gleiche semantische Interpretation, sondern auch die gleiche Tiefenstruktur wie der Ausgangssatz haben" (Steinitz, $30)$.

9 - Zu dem Faktor "aktuelle Satzperspektive" im Rahmen des hier angewendeten Beschreibungsmodells s.u. bes. Kap. 3.2, S. $86 \mathrm{ff}$.

10 - $\mathrm{Zu}$ der Negation und ihre Verwendung im operativen Test s.u. bes. Kap. 5.1, S. $185 \mathrm{ff}$.

11 - Man vgl. dazu in: Explizite Beschreibung der Sprache und automatische Textbearbeitung, II, Praha 1975: "Es wurde gleichfalls darauf hingewiesen, daß man sich fragen muB wenn sowohl die Standardtheorie (d.h. die mit der Interpretationssemantik) als auch die mit der generativen Semantik genau so gut die Aufgabe der Bestimmung einer Menge von grammatisch richtigen Sätzen einer Sprache wie die Aufgabe der zuweisung von geeigneten Strukturcharakteristiken erfüllen - wie jede von ihnen einer dritten Aufgabe genügt, nämlich, auf welche Weise sie die Lautgestalt des Satzes mit dessen Bedeutung verbinden und inwiefern diese verbindungsweise der Kompetenz eines "native speaker's" nahesteht" (loc. cit. $16 \mathrm{fl}$.

12 - zu der Notwendigkeit der Unterscheidung der Untersuchungsebenen vgl. Panevová (1978b), 227: "We have shown elsewhere ... that when speaking about obligatoriness 
of a participant we must take into consideration the level concerned, because the semantic and the syntactic obligatoriness are two different notions. We deal with the "semantic structure" of sentence here (i.e. the tectogrammatical level in the sense of sgall $^{3}$ and our criterion characterized below is intended for this level."

13 - Eine klassische Prager Anleitung zur Handhabung operationeller Untersuchungsmethoden kommt in folgender Stellungnahme Sgalls zum Ausdruck: Eine "wissenschaftliche Beschreibung der Sprache kann nicht vollständig sein, wenn sie nicht die beiden Richtungen, die beiden operationsverfahren einschließt: erstens die, die den Formen Bedeutung zuschreiben und so die Möglichkeit des Textverständnisses geben (einschließlich der Kriterien für eine Auswahl bei mehreren gegebenen Funktionen im Fall der Homonymie - soweit sie im System der Sprache gegeben sind, und nicht durch außersprachliche Bedeutungen), wie auch jene, die den Bedeutungen Formen zuschreiben (einschlieblich sprachlicher Kriterien für die Auswahl einer Form aus synonymen Formen für eine bestimmte Funktion)" Sgall (1977) $11 \mathrm{f}$.

14 - Nach Sgall weisen im besonderen die Probleme, die mit der Untersuchung der Satzsemantik verbunden sind, auf die Nähe zwischen logischer Semantik und linguistischer semantischer Forschungen, wenn sie nicht als lexikalische Bedeutungslehre verstanden werden. Unter seinen Arbeiten, die eine Annäherung der semantischen Satzrepräsentation an das Prädikatenkalkül vorschlagen, ist auch die von Bartsch erwähnt, von der es hier heiBt: "Eine linguistische Anwendung der Modelltheorie (als einer für die modalen Logiken geeigneten semantischen Grundlage) findet man zum ersten Mal in der Bearbeitung der Adverbiale bei Bartsch (1972)." Sgall, P., Stellung und Aufgaben der algebraischen Linguistik (Einführungsstudie), II Bulletin ML, 23 (1975), 68. 
15 - Eine Uberprüfung der von Bartsch vorgeschlagenen Klassifikation der Prädikatsvariablen an breitem empirischen Material liegt in der Arbeit von Krause vor; vgl. Krause, J., Untersuchungen über das Verb "geschehen", Tübingen, 1977.

16 - Zu der Diskussion über Prädikate und ihre Klassifizierung in der Logik, besonders im Hinblick auf die Probleme, die zu einer möglichen Prädikatsklasse "Ereignis" zur Sprache gebracht werden, verweise ich auf Tugendhat, E., Vorlesungen zur Einführung in die sprachanalytische Philosophie, Frankfurt, 1976, bes. $453 \mathrm{f}$.

17 - Die im folgenden dargelegten Untersuchungen $z u$ Lokaladverbialen ( $\mathrm{s}$. Kap. 3 , S. $58 \mathrm{ff}$ ) ergaben, daß für die linguistische Analyse die Frage der Wortstellung der Advb im Satz einbezogen werden muß; mit ihr scheint ein "surplus" sprachlicher Bedeutung verbunden $z u$ sein, das mit prädikatenlogischer Darstellung nicht erfaßt wird (s.u. Kap. 3.2, S. 86 ff).

18 - Für die linguistische Analyse der durch das Adverbial in Relation gesetzten Satzglieder halte ich es - im Hinblick auf die syntaktischen Verhältnisse - für zweckmäßig, das Adverbial als Prädikat aufzufassen und das Glied, wovon es prädiziert wird, als ein in der Regel transformationell zu erschließendes Satzglied; vgl. Bartsch $z u$ den "relationalen Adverbialen": "Die Relationen zwischen den Ereignissen oder Umständen sind inhaltlich bestimmt (kausal, final, konzessiv, adversativ, temporal ...). Wegen dieser inhaltlichen Bestimmtheit - ohne die es sich gar nicht um objektsprachliche Prädikate handeln würde, lassen sich die Relationen zusammen mit einem ihrer Argumente als ein einstelliges Prädikat auffassen, das über das Ereignis, das durch den Satz im anderen Argument beschrieben wird, prädiziert werden kann. Dieses einstellige Prädikat ist das jeweilige Adverbial: z.B. $\phi_{c=c a u s}$ (Er ist krank. , ...) ... wegen seiner Krankheit" (106). 
19 - zu dem hier angesprochenen Problem der sprachlichen Mehrdeutigkeit s.u. Kap. 4.4, S. $166 \mathrm{ff}$.

20 - Es wäre zu erwarten, daß der Negations-Test den Prädikations-Test ergänzt in dem Sinn, daß er bei Negation des ermittelten Prädikats das erhaltene Ergebnis bestätigt oder - wie der Prädikations-Test - nicht anwendbar ist. Die Untersuchungen an Sätzen mit freien Adverbialen zeigen jedoch, daß zwischen der Negationsmöglichkeit des Prädikats des Oberflächensatzes und der des dem Adverbial zugrundegelegten Prädikats unterschieden werden muB, da die verschiedenen Klassen der Advb hier unterschiedliche Regelmäßigkeiten aufweisen; vgl. ausführlicher dazu Kap. 5 "Modaladverbiale", S. $183 \mathrm{ff}$.

21 - Zu diesen Unterschieden vgl. u.: Lokaladverbiale, S. 69 f, 125 f; Temporaladverbiale S. 137 f; Modaladverbiale S. $189,194 \mathrm{f}$.

22 - Bei diesem Analyseschritt werden - insbes. bei den lokalen Advb - Beziehungen zwischen Prädikation und Wortstellung bzw. aktueller Satzperspektive sichtbar, s. Kap. 3.2, S.86 ff.

23 - Zu diesem "gordischen Knoten" in der Linguistik hat Sgall wiederholt stellung bezogen - so vertritt er zu dem "Angelpunkt" der Darbietung von Forschungsergebnissen in einer wissenschaftlichen Grammatik den Standpunkt, daß es prinzipiell zwei Möglichkeiten gibt: von der Form zur Bedeutung und umgekehrt, wobei für den ersten weg die lange Tradition der wissenschaftlichen sprachlichen Untersuchungen spricht; andererseits aber - so Sgall - wiegen die Argumente schwer, die darlegen, daß "die tatsächliche Basis des grammatischen systems die semantische struktur des Satzes ist, und daß die Besonderheiten der Oberflächensyntax, der Morphologie usw. als kombinatorisch bedingte Ausdrucksformen dieses Systems erklärt werden müssen" (Sgall (1977), 11). 
ANMERKUNGEN ZU KAPITEL 3:

1 - Diese Erscheinung ist in der Literatur des öfteren behandelt worden. Sehr aufschlubreich ist der Artikel von Kržižkova, E., Adverbial'naja determinacija so znałeniem mesta $i$ napravlenija (Opyt transformacionnogo analiza) VJa, 1967, 2, 32-48. Vgl. die Literaturangaben dort. Ungeachtet des vergleichbaren transformationellen Rahmens in den Analysen von Křížková und denen in dieser Arbeit weisen die Ergebnisse zu der Bestimmung der Tiefenstruktur (vgl. die Derivationsschemata) wie auch zu dem Erklärungszusammenhang für die Bedeutungsveränderungen durch die Wortstellung (s. Kap. 3.2, S. $86 \mathrm{ff}$ ) wesentliche Unterschiede auf.

Einen anderen Standpunkt vertritt Uhlířová, nämlich, daß "optionale Konstituenten" die Stellung im Satz ändern können, ohne daß dieses einen "substantiellen Einfluß" auf die aktuelle Satzperspektive habe, vgl. Uhlířová, L., Optional Constituents in Theme-Rheme Structure, in: P. Studies M.L., 5, 1976, 309-320, bes. 315 ff. Dagegen macht von den neueren Arbeiten Koktova auf die Beziehungen zwischen der Stellung der Advb und der Thema-Rhema-Gliederung aufmerksam, vgl. Koktová, (1977), bes. 48.

2 - Auf Besonderheiten bei dieser Stellung von Advb ${ }_{10 c}$ im zusammenhang mit der Bedeutung des Satzes und der ThemaRhema-Gliederung wies $P$. Adamec hin, indem er auf die Unterschiede von Sätzen wie "(21) Deti igrajut vo dvore." und "(22) V dvore igrajut deti." aufmerksam macht. Die hier untersuchten Sätze bestätigen die Feststellung von Adamec, das bei stellung am Satzende, dabei im besonderen in der Funktion des Rhema das freie Lokaladverbial sich "leicht" in ein "Prädikat periphrasieren" läßt, so Adamec zu (21): "(25) Igra detej/proischodit vo dvore." Adamec, P., Aktual'noe členenie, glubinnye struktury i perifrazy, in: Danes, F., Papers on Funcional Sentence Perspective, 1974, $194 \mathrm{f}$. 
Auf die "sekundäre Prädikation" und auf sie als möglichen Faktor für die Bedeutungsstruktur des Satzes machte ebenfalls D. Weiss aufmerksam. Weiss, D., Nichtrestriktive Attribution vs sekundäre Prädikation: Zwei Typen von "Backgrounding", in: Slavica Helvetica, Bd. 12, 1978, 251-271.

3 - In der Terminologie bediene ich mich der Begriffe, die von der Prager Schule und den in ihrer Tradition stehenden Arbeiten verwendet werden; vgl. Vachek, J., Dictionnaire de linguistique de l'Ecole de Prague, Utrecht, 1960.

4 - Vgl. im Unterschied dazu das Verfahren bei Ǩ́ížkova, die die Bedeutungsunterschiede, die bei Stellungswechsel von Advb im Satz zu beobachten sind, im wesentlichen durch den Kontext bedingt ansieht (op. cit. 36), diese Bedeutungsunterschiede dennoch transformationell $z u$ beschreiben versucht und damit nicht vermeiden kann, mehrere Ableitungsschemata für Advb ${ }_{10 C}$ $z u$ formulieren (op. cit. 35-38).

5 - Die Besonderheit der lokalen Adverbiale besteht darin, daß sie als Prädikate über verschiedene Individuenbereiche ausgesagt werden können - über "Dinge" wie auch über "Ereignisse", s. dazu u. S. 66 f.

6 - zu der Strukturierung von Aussagen und ihrer zuordnung zum Sprachsystem vgl. Sgall, P., Zum Stand der Thema-RhemaGliederung in der Tschechoslowakei, in: Theoretische Linguistik in Osteuropa, 1976, 163-182; zu sprachlichen Formen, die auf Faktoren der Kontextabhängigkeit zurückzuführen sind, vgl. Kovtunova, I., Porjadok slov $i$ aktual'noe Xlenenie predloženija, Moskva, 1976, bes. Kap. II.

7 - Ausführlicher zum Negations-Test s. u. Kap. 5.1, $185 \mathrm{ff}$. 
8 - s. dazu S. 84 f Beispiele (99)-(101).

Zur Demonstration von Sätzen mit Advb ${ }_{10 c}$ bei Passiv-Tf wird hier (11) vorweggenommen (s.u.).

9 - Vgl. Steinitz, R. (1971) und meine Ausführungen dazu S. $30 \mathrm{f}$ und S. $71 \mathrm{ff}$; Koktová, E. (1977).

10 - In der Literatur findet man die Behauptung, daß zwischen $\mathrm{S}^{1}$ und $\mathrm{s}^{2}$ TS Tempuskongruenz bestehe (vgl. Bartsch, 1972, 107, bes. 125; Steinitz, 1971, 30 f). Das ist wohl so allgemein nicht zutreffend. Man vgl. bei (33) Cerkov' V.B. $\left\{\begin{array}{c}\text { byla } \\ \theta\end{array}\right\}$ v Moskve.

Die Regel, nach der Tempuskongruenz erforderlich ist oder nicht, hängt offensichtlich von verschiedenen Faktoren $a b$ : zum einen von der Klasse der Objekte, wenn die PP loc über das Objekt eine Aussage macht. Handelt es sich nẳmlich um effizierte Objekte, ist Tempuskongruenz nicht erforderlich (vgl. 33), handelt es sich um affizierte Objekte, ist sie die Regel. Eine Einschränkung erfährt auch diese Regel bei bestimmten Verbklassen - bedingt wohl durch semantische Merkmale; man vgl. (25), (30): Pamjatniki napominajut o partizanach. Partizany byli v selach $i$ lesach Belorussii.

11 - Kržižkova, E. (1967); Panevová, J. $(1978$ a) 9 f; Steinitz, R. (1971) 31 und $34 \mathrm{f}$.

12 - anders bei Temporal- und Modaladverbialen, siehe Kap. 4 , 2.B. $138 \mathrm{f}$ und Kap. 5, $194 \mathrm{f}$.

13 - zur Definition von "merkmallos": bei vorliegender Ubereinstimmung von "kommunikativer Dynamik" und "kommunikativer Information"; so in Firbas, J., On Defining the Theme in Functional Sentence Analysis; in: Travaux linguistiques de Prague, I, 267-280, Praha, 1964. 
14 - zu möglichen Restriktionen der Prädikation s. unten Kap. 3.2, $87 \mathrm{f}$; wie auch die weiteren Ausführungen unten Kap. 3.2.1, besonders $96 \mathrm{ff}$.

15 - Die TG hat auf die Prädikation durch andere Satzglieder als das des konjugierten Verbs aufmerksam gemacht; vgl. die Ableitung bestimmter Klassen attributiver Adjektive; die Bescheibung zusammengesetzter Nomina (Brekle, 1970); vgl. neuerdings Weiss, D. (1978). Bei Weiss wird die Prädikation z.B. durch Adverbialpartizipien - wobei auch das Problem der Adverbiale gestreift wird - als "sekundäre Prädikation", "Backgrounding" eingestuft. Es ist jedoch zweifelhaft, ob eine solche Hierarchisierung der Prädikate den sprachlichen Gegebenheiten angemessen ist. zu zweifeln gaben die Beobachtungen an temporalen Adverbialen AnlaB; vgl. unten $S$. $150 \mathrm{ff}$.

16 - zu der Frage von weiteren Oberflächenstrukturen s. die weiteren Ausführungen und Kap. 3.1.3, $79 \mathrm{ff}$.

17- Dabei können bei der Anwendung des Prädikations-Tests Oberflächensätze entstehen, die an die Grenze der Akzeptabilität stoßen, so u. (41). Aber auch diese Beispiele bestätigen den metasprachlichen Beschreibungszusammenhang.

18 - In (8) und (40) handelt es sich um themalose Sätze. Es scheint, daß für themalose Sätze Anfangsstellung der Adverbiale typisch ist (s. unten Kap. 3.2.3, $117 \mathrm{ff}$ ). Vgl. dazu Arutjunova, N., Predloženie i ego smysl, Moskva, 1976, bes. Kap. IV, OtnoSenija èkzistencii. Bei (40) ist auch die systembedingte Ellipse zu beobachten: "Na fone" ... kann auch als nähere Bestimmung zu "strast'" verstanden werden. Diese Mehrdeutigkeit ist Folge zum einen der sprachlich nichtexpliziten Beziehung der lokalen Prädikation zu ihrem Argument, zum anderen der Prädikatonsmöglichkeit lokaler 
Prädikate über Argumente unterschiedlicher kategorialer Bereiche.

19 - Adverbiale wie "v poezdkach", vgl. auch (48) "na konferencii" können lokal wie temporal verstanden werden, $s$. hierzu unten "Temporale Adverbiale", bes. $172 \mathrm{ff}$.

20 - Vgl. oben's. 242, Anm. 2 die grammatische Beschreibung, die bereits Adamec vorgeschlagen hatte, allerdings ohne auf die systematischen zusammenhänge einzugehen. zu der Frage der Hierarchie der Prädikate s. unten S. $73 \mathrm{ff}$, wie auch $u$. Anm. 27 .

21 - Die Definiton der TS (S. 48 ff) als die Beschreibungsebene, die die syntaktischen und die semantischen Relationen der Satzglieder zueinander in Beziehung setzt, erlaubt es, zwischen der Hierarchie syntaktischer und der semantischer Strukturen zu unterscheiden. Die hier dargestellten syntaktischen Ableitungsprozeduren stehen damit nicht im widerspruch zu der Darstellung der semantischen Verhältnisse (Schema 1). Meine Beobachtungen ergaben, daß Oberflächenstrukturen des Typs $(50)-(54)$ bei lokalen, temporalen und modalen prädikativen Ausdrücken vertreten sind, daß sie aber besonders häufig in Sätzen mit temporalen Prädikaten vorkommen, was im Zusammenhang damit gesehen werden muß, daß temporale Adverbiale in der Regel über das finite Verb prädiziert werden (s. unten Kap. 4, $128 \mathrm{ff}$ ).

22 - Vgl. dazu oben die einschränkenden Ausführungen im besonderen S. 244, Anm. 10 .

23 - Kriterien für eine eindeutige Abgrenzung von Satzadverbial und relationalem Adverbial für das Deutsche gibt Bartsch (1972), vgl. oben S. $43 \mathrm{f}$. 
24 - Vgl, auch die diesbezüglichen Ergebnisse bei Koktová, E. (1977), 47 f. Sie kommt zu dem Schlus: "that the possible temporal paraphrasing of the optional local adverbials is only a matter of the cognitive content; it will appear that the possible temporal representation is only a fortuitous phenomenon since there are multivarious both grammatical and semantical restrictions which prevent various sentences from allowing for 'temporal paraphrases' ..." ibid.

25 - Anders wohl bei Temporal- und Modaladverbialen, s. dazu Kap. 4, z.B. S. 141 und Kap. 5, z.B. S. $202 \mathrm{f} ; \mathrm{vgl}$. auch Tugendhat, E. (1976) $451 \mathrm{ff}, \mathrm{zu}$ Lokalaussagen im Sinne einer Primärprädikation.

26 - Eine ausführliche Darstellung von $S_{\text {temp }}$ geht über den Rahmen dieser Arbeit hinaus. Dafür wären temp weitere untersuchungen, ausgehend von $s_{\text {temp }}$ notwendig. Es sei verwiesen auf Wunderlich, D., Tempus und zeitreferenz im Deutschen, München, 1970.

27 - $\mathrm{zu}$ der Hierarchie der Prädikate, die mit dieser Bedeutung explizit gemacht wird, s. unten Temporaladverbial, Kap. 4, im bes. 4.2 , S. $\frac{1}{2} 50 \mathrm{ff}$. Die Frage der Hierarchie der Prädikate von $S^{1}$ und $S^{2}$ ist m.E. - wie schon angeführt für die sprachlichen Strukturen mit Lokaladverbialen nicht relevant. Die Untersuchungen an dem empirischen Material lassen in dieser Hinsicht keinerlei Restriktionen in Form etwa von Stellungsregeln erkennen; diese sind allerdings für die Thema-Rhema-Gliederung der Aussage von Bedeutung, s. Kap. 3.2, im besonderen S. $100 \mathrm{f}$.

28 - s.o. zu Steinitz (1971) und Koktová (1977) - auch das Kriterium von Koktová der "Beweglichkeit" bzw. "Unbeweglichkeit" von $\mathrm{NP}^{1}$ ist von sekundärer Bedeutung (loc. cit. 50 f). 
29 - Vgl. auch die Ausführungen zu PP ${ }_{10 C}$ im Zusammenhang mit der Thema-Rhema-Gliederung, s. unten Kap.3.2.2.1, $106 \mathrm{ff}$; vgl. auch Kržižkova, E. (1967) 35 .

30 - Vgl. dazu oben S. $68 \mathrm{f}(50)-(54)$ und Anm. 21 .

31 - zu der Beziehung von $s^{1}$ und $s^{2}$ in Sätzen mit Modaladverbialen $s$. unten Kap. 5 "Modaladverbiale" bes. $S$. 194 ff und $208 \mathrm{ff}$.

32 - Unter den $z u$ beobachtenden Fällen der Häufung von Adverbialen läßt sich nur ein Teil in der für $(70-75)$ vertretbaren Weise als sukzessive Aneinanderreihung von Aussagen und formal als Beziehung von $\mathrm{Tf} z \mathrm{z}$ anderen $\mathrm{Tf}$ beschreiben. Bei Kombination von Adverbialen, z.B. lokalen, temporalen und lokalen etc. liegen auch andere und nicht immer eindeutige Relationen vor, auf die ich hier nur hinweisen kann, z.B. die der Aufzählung:

(76) Pervye zvezdy zazigalis' na rozovom nebe nad nepodviznymi verchuskami lip.

Bei Verben, die aufgrund ihrer subkategorisierung einer Adverbialergänzung bedürfen, ist der syntaktische Status der Adverbiale bei Kombinationen nicht eindeutig. Bei folgenden

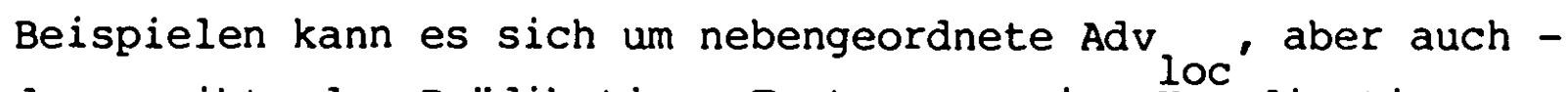
das ergibt der Prädikations-Test - um eine Koordination von Advb ${ }_{\text {loc }}$ und Adv ${ }_{\text {loc }}$ handeln, wobei für die wortfolge die Regeln $z u$ beachten sind, die unten Kap. 3.2 ( $86 \mathrm{ff}$ ) dargestellt werden.

Man vgl.:

(77) ... provel $v$ Peterburge na sluzbe.

(78) ... provel na službe $v$ Peterburge.

(79) Pered nim na prigorke tjanulas' ... dereven'ka.

(80) Na prigorke pered nim tjanulas' ... dereven'ka.

zu "Kombinationen" bzw. "Subordination" von Adverbialen verweise ich auf: Steinitz (1971); Bartsch (1972); Bennett, 
D.C., Spatial and Temporal of English Prepositions, London, 1975, bes. $30 \mathrm{f}$.

33 - s. Oben Kap. 2, S. 28 und Anm. 7 dazu.

34 - Zu den Beziehungen zwischen Wortbildung und Tf $s$. "Explizite Beschreibung der Sprache und automatische Textbearbeitung", II, 1975, 15 f, 23; Panenová (1978a) ll f.

35 - zu der Neutralisierung von attributiven und prädikativen Beziehungen in Folge von Ableitungen deadjektivischer Nomina s. Zolotova, G. (1976), O sintaksiČeskich svojstvach imen kaðestva, in: Zolotova, G. (Hrsg.), Sintaksis i stilistika, Moskva, 1976, 130-160.

36 - Vgl. Slovar' (AN) (1950 ff), t. 9, Sp. 342, Eintrag zu "peručest'": "svojstvo i kað̌estvo peruð̌ego".

37 - In einer Vielzahl von Beispielen sind es semantische, pragmatische oder sonst kontextbedingte Faktoren, die die Mehrdeutigkeit auflösen. Dafür ist (92) ein Beispiel, das syntaktisch (89) entspricht: (92) ... posle ot''ezda Lavreckogo javilsja Panšin, kotorogo Varvara Pavlovna prosila ne zabyvat' ee $v$ uedinenii. Der Leser von "Dvorjanskoe gnezdo" wei $\beta$ aufgrund des Kontextes, daß sich "v uedinenii" auf Varvara Pavlovna bezieht. Diese Faktoren werden in meiner Arbeit nicht behandelt, da sie sich weitgehend Generalisierungen entziehen.

38 - Erwähnung finden soll hier ein wohl in die Grammatik einzuordnender Faktor, der disambiguierend bei den Prädika-

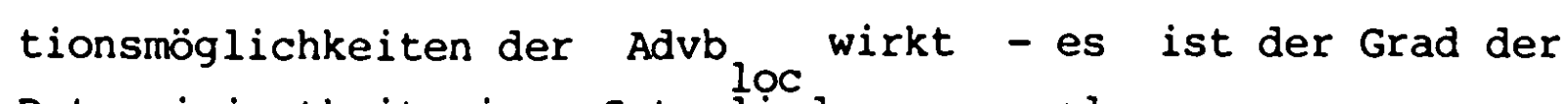
Determiniertheit eines Satzgliedes; man vgl.:

(93) Varvara Pavlovna nanjala sebe luð̌suju karetu v gorode. im Unterschied zu:

(94) Varvara Pavlovna nanjala sebe karetu v gorode. 
39 - aus: Krebs, D., Die Passivfunktion von -sja im russischen Satz (Versuch einer syntaktisch-semantischen Analyse), Saarbrücken, 1974, Dipl.-Arbeit, S. 24 f.

40 - Zimmermann, I. (1978), 75, vgl. ihr Beispiel:

(2a) $V$ ètoj knige avtor ${ }_{4}$ izlagaet istoriju stolicy ${ }_{2}^{\prime}$

(2 ) $\mathrm{V}$ ètoj knige ${ }_{4}$ avtorom ${ }_{1}$ izlagaetsja istorija stolicy ${ }_{2}$ '

(2b) $\mathrm{V}$ etoj knige izlagaetsja istorija stolicy ${ }_{4}{ }^{\prime}$

(2) Eta kniga izlagaet istoriju stolicy ${ }_{2}$.

(Die Zahlenindices symbolisieren die hierarchische ordnung der Aktanten und Determinanten des Verbs in seiner "Ausgangsdiathese".) Vgl. die Ausführungen oben Kap. 2.1.5, S. $49 \mathrm{f}$. Auf die Verwendung der Advb ${ }_{10 c}$ in diesem "sehr produktiven" Typ unpersönlicher Sätze macht die Grammatika (1970) aufmerksam; vgl. S. 632 u.a. die Beispiele: "V odesskojoblasti forsiruetsja/ odesskaja oblast'forsiruet// s d a zu z e rna; Na plenume obsuż dalis'/Plenum obsuždal vaznye v o p r o s y."

41 - Vgl. dazu im Sinne von "geteiltem Wissen", Labov, W., Sprache im sozialen Kontext, Königstein/Ts, 1978, Bd. 2, 6 ff.

42 - Darauf machte auch die Untersuchung $z u$ dem Satzkonnektor von $\mathrm{s}^{1}$ und $\mathrm{s}^{2}$ aufmerksam - s.o. Kap. 3.1.2, 76 f. Ich beschränke mich auf die Untersuchung geschriebener Texte, die in der Regel "expressiv neutral" sind. Die funktionale Satzperspektive wird mit Hilfe von wortstellungsregeln diskutiert; die Intonation wird nicht experimentell behandelt, ist jedoch insofern einbezogen, als es sich um Sätze mit "normaler" Intonation handelt.

43 - Für Literaturangaben verweise ich auf die oben S. 242 f zitierten Anm. 1-3,6. Weiter stütze ich mich auf folgende 
Arbeiten: Dahl, ర., Topic and Comment. A Study in Russian and General Transformational Grammar, Stockholm (1969); Adamec, P., Porjadok slov v sovremennom russkom jazyke, Praha (1966); Sgall, P. Hajičová, E., Benešová, E. (1973).

44 - Bei der Uberprüfung der Gliederungsmöglichkeiten an Oberflächensätzen bleiben die Unterschiede zwischen adverbialer und attributiver Beziehung nicht immer explizit (z.B. bei $\left.Q_{4}\right)$. Das widerspricht jedoch nicht den tiefenstrukturellen Analysen, sondern ist durch die oberflächenstrukturelle Form bedingt.

45 - Bekanntlich können zu jedem kontextfreien Satz in der Regel mehrere kontextabhängige Formen gebildet werden. Es werden hier nur die angeführt, die zu der gestellten Frage Aufschlüsse geben.

46 - Die Pronominalisierung als Mittel der Organisation der aktuellen Gliederung muß hier aus Gründen der Themabeschränkung unberücksichtigt bleiben. Belegstellen weisen jedoch darauf hin, daB die Ausführungen in dieser Arbeit z.B. über PP als Rhema proprium in Sätzen mit Pronomina bestätigt werden; man vgl.:

(107) No o tom, kto mnogie iz nich "somnitel'nye", direktor umolčal. Ne pokazal on ètogo / i

v soprovoditel'nych dokumentach.

PP ${ }_{\text {loc }}$ ist hier nähere Bestimmung zum finiten Verb. Dagegen wird in

(108) Kak ja spešil! ... branilsja, kriłal, treboval lošadej, daže bujanil / iz-za lošadej na stancijach $\ldots$ 
dadurch, daß das schon bekannte Nomen "loładi" nicht durch ein Pronomen ersetzt wird, die PP ${ }_{\text {IOC }}$ vorrangig als nähere Bestimmung zur Verbergänzung verstanden.

47 - Es könnte der Einwand erhoben werden, daß das Testergebnis mit $\mathrm{Th}_{3} / \mathrm{Rh}_{3}$ : "O partizanach pamjatniki napominajut/v selach $i^{3}$ lesach Belorussii" nicht mehr dem $z u$ untersuchenden Satz $S$ "O partizanach napominajut pamjatniki" + PP loc (hier "v selach i lesach Belorussii") entspricht. Tatsächlich wird dieses Testergebnis nicht primär für die Problemlösung verwendet, sondern nur in Gegenüberstellung $z u$ "O partizanach napominajut pamjatniki / $v$ selach $i$ lesach Belorussii" $s \cdot Q_{7}$; weiter dazu unten 5.98 die Aussagegliederung "b" der Beispiele.

48 - Auch möglich: Kogda izuČal Dal' byt ...? zu der Mehrdeutigkeit von PP als Lokal- und Temporaladverbial s.u. Kap. "Temporale Adverbiale", S. 172 ff.

49 - Für unsere Fragestellung können wir für eine Menge von Sätzen (hier (42), (46), (48), (110) diese beiden wortfolgemöglichkeiten innerhalb des Thema als Varianten betrachten. Das gilt nicht für (4); u.a. s. dazu unten.

50 - Die Frage nach den Voraussetzungen für diese sich widersprechenden Ergebnisse bei der Anwendung des ThemaRhema-Gliederungs-Tests mit $Q_{6}$ überschreitet den Rahmen dieser Arbeit. Es wäre die Frage danach, welche prädikate welchen Argumenten aufgrund unseres wissens von der Welt zugesprochen werden.

51 - Zu dem Problem der "Kontaktstelle" von Satzgliedern im Zusammenhang mit der funktionalen Gliederung des Satzes s. Krylova, O., Chavronina, C., Porjadok slov v russkom jazyke, Moskva (1976), 49. 
52 - Die Stellung der PP ${ }_{10 C}$ vor dem finiten VB wird u. Kap. $3.2 .2,106$ ff untersucht.

53 - Mit der Bedeutung der "Grenze" von Thema und Rhema für die objektsprachliche Beschreibung wie auch mit der Frage ihres Stellenwertes im Rahmen einer linguistischen Theorie hat sich wiederholt P. Sgall auseinandergesetzt, vgl.z.B. Sgall (1976); ausführlicher Sgall, Hajicová, Benešová (1973) Kap. II, 4. Sgall stützt sich bei seiner Argumentation für den Nachweis, daß die T-C-A dem Sprachsystem zuzuordnen ist, im besonderen auf Untersuchungen zum Scopus von Negation und Quantoren. Die PP sind in dieser Hinsicht m.W. bisher nicht untersucht worden.

54 - Die Verwendung dieses Satzes mit dieser T-C-A in Texten mag angezweifelt werden können. Das Beispiel dient vornehmlich der Illustration der Probleme, die die Belege (114 ff) aufwerfen. Es ist jedoch folgender Kontext denkbar: "Voroustvo $v$ centre bol'కogo goroda. Policija vskryla neskol'ko vorov. Odnich pojmali, a tot $v$ tolpe ljudej/ iscez." Originalbelege s. ff Beispiele.

55 - Aus Gründen der Uberschaubarkeit der $2 u$ untersuchenden Faktoren ist die Analyse auf expressiv neutrale Aussagen beschränkt. Jedoch soll Erwähnung finden, daß auch in expressiven Aussagen eine Abhängigkeit der Funktion der PP loc von der Grenze $z$ wischen Thema und Rhema vorliegt: man vgl.: (122) A nu vzdumaj my / na smertnom odre / zavescat' komu-to Kurskij vokzal ili Ostankinskuju telebasnju.

56 - Zu PP und Relativsatz als syntaktisch synonyme Oberflächenstruktur s. Kap. 3.1 .2 bes. S. $77 \mathrm{f}$ und $z u$ Kap. 3.2.2.2, bes. $z u 115 \mathrm{f}$, Anm. 62, die auf ein weiteres Merkmal, das mit der stellung der PP verbunden ist, hinweist: die Graduierung der "kommunikativen Dynamik" . 
57 - Zu Sätzen mit PP ${ }_{10 c}$ nach dem finiten Verb s. unten Kap. $3.2 .2 .2,114 \mathrm{ff}$.

58 - Die Kleinbuchstaben ( $\mathrm{rh}$ ) werden als Symbole für abgeleitetes Rhema verwendet, das im komplexen Satz sowohl im Bereich des Thema als auch des Rhema vorkommen kann. Vgl. dazu: Beneš, E., Thema-Rhema-Gliederung und Textlinguistik, in: Studien zur Texttheorie und zur deutschen Gramatik, $1973,42-62$.

59 - Vgl. z.b. Sgall, Hajičová, Benešová (1973), 47; Firbas (1964); vgl. auch Jaksche (1978), zu den "zwei kommunikativen Zentren".

60 - Zu der Bewertung der kommunikativen Dynamik am Anfang der Aussage, s. Jaksche, loc. cit. S. 83.

61 - Peškovskij wies schon auf die auffallende Wortstellung von - in seiner Terminologie - "schwach regierten" und "stark regierten" Verbergänzungen hin. Seine Beobachtungen illustriert er mit folgendem Beispiel: "In den Wortverbindungen pišu zapisku und pišu tebe na užasnoj bumage, užasnym perom, kotoroe mne edva udalos' dostat' u tvoego večno zaspannogo, dolgo nið̌ego ne ponimavšego sožitelja, pri polnoj potti temnote, potomu tto nekogda dožidat'sja, poka on soblagovolit zazex' lampu, zapisku - ist die Abhängigkeit des Wortes 'zapisku' von dem Wort 'pisu' in beiden Fällen gleich klar." Peškovskij, A.M., Russkij sintaksis v nauðnom osveščenii, Moskva (1934), 256.

62 - Weitere Argumente für die Relevanz der hier angenommenen Graduierung der kommunikativen Funktion der PP $10 c^{\prime}$ die deren Primärfunktion - die Prädikation - abschwächt, gibt die Position der PP ${ }_{10 C}$ z.B. zwischen Attribut und Beziehungswort; man vgl.: 
(137) Portret byl velikolepen! - Risoval pervyj $\underline{\mathbf{v}}$ gorode chudožnik.

(138) Portret byl velikolepen! - Risoval pervyj chudoźnik $v$ gorode.

Diese Regel kann unter bestimmten Bedingungen eingeschränkt sein, s.u. "Temporale Adverbiale", S. 169 u. 181.

63 - Die andere Wortfolgemöglichkeit - Anfangsstellung der PP unter Beibehaltung der Reihenfolge NP VP - kommt unten in doc Sätzen (159) - (161) zur Sprache.

64 - z.B. Sachmatov, A.A., Sintaksis russkogo jazyka, Moskva, 1925, \$ 159 (S. 159) zitiert nach Reprint, The Hague, 1963. Mathesius, V., A Functional Analysis of Present Day English in a General Linguistic Basis; in Vachek, J. (Hrsg.) The Hague-Paris (1975), 82,f; Firbas (1964), 268; Kovtunova (1976), 47 f; Arutjunova (1976), 229 ff.

65 - Die Aussage auf dem Hintergrund der 1. Fragemöglichkeit setzt Kontextabhängigkeit voraus, d.h. so verstanden ist "Zažigalis' (na rozovom nebe) / pervye zvezdy" die syntagmatisch starke Form zu (19).

66 - Satzpaare, denjenigen entsprechend, mit denen wir hier operieren, um die unterschiedlichen Funktionen von PP in Anfangs- und Endstellung $z u$ bestimmen, sind in ${ }^{10 c}$ der linguistischen Literatur wiederholt diskutiert worden, um unter Hinweis auf logische Untersuchungen den Begriff der "H i e r a r c h e de $r$ Bed e u t u g e n" (Jakobson, 1935) zu veranschaulichen; vgl. Jakobson, R., Form und Sinn, 1974, 87 f; Sgall, Hajitová, Benešová (1973) 147 f. 
ANMERKUNGEN ZU KAPITEL 4:

1 - Von den älteren Arbeiten liefert für das Russische eine repräsentative Aufzählung der morphologischen Formen und lexikalischen Besetzungen für zeitbezeichnungen: Robert, C., Contribution à l'étude de quelques compléments de temps en russe moderne, Paris, 1964.

2 - Für unsere Problemstellung waren dabei von Bedeutung: Steube, A., Uber die Beziehungen der Zeitadverbien zum Satz und ihre Selektionsbeziehungen $z u$ den relevanten Elementen im Satz, im: ASG-Bericht Nr. 3, Berlin (1969); Wunderlich, D. (1970); Bartsch, R. (1972); Bennett, D. C. (1975); Krause, J. (1977); Panevová, J. (1978a); KuČera, H., Trnka, K. (1975).

3 - $\mathrm{Zu}$ der Diskussion über obligatorische adverbiale Ergänzungen, die in der Regel temporal sind, bei Verben wie "stattfinden" vgl. Steinitz (1971) $26 \mathrm{ff}$; dagegen Bartsch (1972) 331 ff; die Diskussion wurde wieder aufgegriffen von Krause (1975).

4 - Es fällt auf, daß Zolotova (1973) mit Nachdruck an die feinsinnigen Ausführungen Sachmatovs, Sintaksis russkogo jazyka (1925), (Repr. 1963) zu den Adverbialen erinnert (vgl. Zolotova, 1973, 246, 250, 298). Es ist interessant, bei Sachmatov ( $\$ 460$ - nicht 449! - und die Anm. dazu) über "Reljativnoe dopolnenie predložnoe" nachzulesen. $\mathrm{zu}$ der Bestimung der temporalen Adverbiale schreibt Zolotova, gestützt auf Šachmatov: "temporal'nye rasprostraniteli vzaimodejstvujut $s$ kategoriej vremeni $v$ predikativnoj osi predloženija" (246).

5 - Bartsch (1972), 118; Katz, (1972), 199 - 260, bes. 213; Steinitz (1971), 97; Wunderlich (1970), $280 \mathrm{ff}$. 
6 - Von neuesten Handbüchern zum Russischen vgl. dazu Grammatika (Praha) (1979), Bd. 2, 763 - 773.

7 - Vgl. für das Deutsche Krause (1977), 29 - 31.

8 - Vgl. Wunderlich (1970), 283: "Es ergibt sich also, daß die (+ Dauer)-Zeitangaben sich voll in das allgemeine Paradigma der Maßangaben fügen, während sich die (- Dauer)zeitangaben durch einige Besonderheiten auszeichnen. Dies kann damit zusammenhängen, daß die (+ Dauer)-Angaben ... eine einfache Zuordnung zu Maßskalen leisten; mit den (- Dauer)Angaben ist jedoch eine zuordnung zu einem Positionssystem, ausgedrückt durch indizierte Maßskalen, verbunden."

9 - Für die Bestimmung der Phrasenstrukturmarker in (F 51) kann sich Wunderlich auch auf Lakoff (1968) berufen, nach dem "auf einer sehr allgemeinen Stufe alle Adverbien (bzw. die relationalen Anteile von Adverbialen) als Verben höherstehender Sätze aufzufassen sind" (Wunderlich, 285).

10 - Nicht wortsemantische Untersuchungen, wie sie im Standardmodell und den darauf aufbauenden generativen Untersuchungen skizziert und durchgeführt wurden. Diese geben Aufschlüsse v.a. über die Satzglieder und ihre möglichen Besetzungen, für die die Regeln der strikten subkategorisierung gelten. Für die Beschreibung der Beziehung von

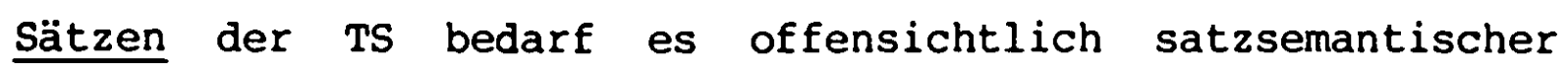
Analysen.

11 - Vgl. ähnlich Wunderlich (1970), 91: "Da sich das zeitkonzept durch Abstraktion aus dem Ereigniskonzept ausbildet, Ereignisse aber als physikalische Prozesse stets von endlicher Dauer sind, müssen auch noch die minimalen zeitelemente primär als endliche zeitintervalle aufgefaßt 
werden. 'Zeitpunkt' ist demgegenüber ein approximatives Konzept."

12 - Vielleicht ist das bei Bartsch auch mit dem Hinweis auf die Mischformen (s.o. S. 132) angesprochen, aber nicht ausgeführt worden.

13 - KuXera/Trnka (1975) widmen ihre Untersuchungen v.a. der Erarbeitung von Merkmalmatrizes der temporalen Ausdrücke ( $s$. 1 - 73). Für ihre Ausführungen über den tiefenstrukturellen Status der Temporaladverbialen (S. 74 - 111) übernehmen sie weitgehend die Vorschläge von Chomsky (1965) und Lakoff (1970) und wenden diese auf Sätze mit mehreren Temporaladverbialen bzw. temporalen Ausdrücken an.

14 - Die formalen Verhältnisse im Russischen sind ein weiterer Grund für die Themaeingrenzung: zu der Klasse Advb Temp guant gehören in der Regeln nicht-präpositionale Ausdrüuake.

15 - Die Verhältnisse liegen anders bei Advb Temp ${ }_{\text {loc }}$ in der Form PP ${ }_{\text {temp }}$ mit NP verb; s.u. S. $144 \mathrm{ff}$.

16 - Es gibt den Fall der Verwendung von PP temp ( ${ }^{N}$ zeit $^{\text {) }}$ als nähere Bestimmung von nominalen Ausdrücken, z.B. als synonyme Oberflächenstruktur zu NP ; man vgl.: "Iskusstvo v 17-om veke pronizano vlijaniem srednevekovych $i$ antičnych tradicij" - "Iskusstvo 17-ogo veka pronizano vlijaniem srednev. i anti $x_{n}$. tradicij".

Aus Gründen der Themabeschränkung habe ich die Frage nach den Bedingungen für die Bildung dieser Ausdrücke nicht untersucht. Mir scheint - entsprechend vergleichbaren Ausdrücken mit Advb ${ }_{\text {loc }}-$, daß es sich um Strukturen handelt, bei denen, bedingt durch die Besetzung der NP, adverbiale und attributive Relationen neutralisiert sind (vgl. O. S. $80 \mathrm{f}$ ). 
17 - Auf das Problem der Wortbildung und dadurch bedingten Bedeutungsveränderungen wurde bereits einschränkend hingewiesen, vgl, oben S. 249, Anm. 34 .

18 - Bei ihrem Versuch, Advb Temp loc auf temporale NS zurückzuführen, stößt Panevová (1978a) auf eine Reihe von sich nicht entsprechenden Elementen in beiden Strukturen. Aufgrund der Ergebnisse unserer Untersuchungen halten wir Advb Temp loc nicht für ableitbar von $S_{\text {temp }}$ sondern $S_{\text {temp }}$ ist eine weitere oberflächenstruktur zum Ausdruck der temporalen Relation von $s^{2}{ }^{2}$ zu s $s^{1}$. Dabei impliziert der temporale NS infolge der anderen sprachlichen Formen - Modus, Tempus, etc. - die zu seiner Realisierung notwendig sind, Kategorien, die Sätze mit Advb Temp loc nicht aufweisen. Besonders interessant sind die Unterschiede, die bei Besetzung der Advb Temp loc mit NP verb durch die Verwendung von zwei Prädikaten bedingt sind (s. dazu S. $194 \mathrm{ff}$ ).

19 - Die Regel gilt - wie oft in natürlichen Sprachen - nicht ohne Einschränkung. Aus unserer Belegsammlung folgt, daß sie mindestens für zwei Fälle modifiziert werden muB: das sind zum einen die schon erwähnten Satzstrukturen mit mehr als einem finiten Prädikat und zum anderen gewisse Satzstrukturen der Sätze mit Advb Temp ${ }_{\text {loc }}$ bei NP verb (s. dazu unten z.B. (88) - (94)).

Rủžička (1980, $180 \mathrm{ff})$ macht auf bestimmte Abhängigkeiten zwischen Stellung und Bedeutung der Adverbialpartizipien im Satz aufmerksam, was u.U. auch für gewisse PP (etwa "pri NP") von Bedeutung sein könnte (s. dazu S. $153 \mathrm{ff}$ ).

20 - Eine indirekte Bestätigung für eine Differenzierung der formalen Strukturprinzipien bei lokalen Adverbialen einerseits und temporalen und modalen (s.u. S. 202 f) andererseits kann auch darin gesehen werden, daß es für Advb und Advb mod breite Substitutionsmöglichkeiten durch das temp Adverbialpartizip gibt; diese bestehen dagegen überhaupt 
nicht für Advbloc • zu der Hierarchie in Sätzen mit Adverbialpartizip s. S. 150 ff.

21 - So auch die Ausführungen bei Zolotova (1973), 298. In ihrem Kommentar zu Sätzen vom Typ "Pri nagrevanii tela rasširjajutsja" übernimmt sie für die Bestimmung der PP von Sachmatov (1925): vyražaet "otnoక̌nija dejstvija k dejstviju."

22 - Die Besetzung der NP, die, von $s^{2}$ dominiert ist, ist in (26) - (30) identisch mit NP, jedoch sind bei dieser Satzstruktur auch andere Möglichkeiten gegeben; s.u. Beispiele (31) - (40).

23 - Auch bei Advb Temp loc mit NP 2 zeit sind verschiedene Relationen $z$ wischen $s^{1}$ und $s^{2}$ möglich. Es scheint jedoch, daß die Form dafür nicht die PP ist, sondern NS als Oberflächenstrukturen; man vgl.: On priłel, potomu tto bylo 7 časov.

24 - Die bedeutungsunterscheidende Funktion, die den Präpositionen hier zukommt, ist nicht nur, wie erwähnt, die Unterscheidungen von Advb Temp loc $_{\mathrm{a}} \mathrm{u}$ Advb Temp quant $z \mathrm{u}$ bezeichnen, und innerhalb der Advb Temp loc die strukturierung des Zeitsystems; sondern hier unterscheiden die Präpositionen Klassen der Adverbialen. Es wird zu zeigen sein (u. S. 172 ff), daß aufgrund der semantischen Mehrdeutigkeit der Präpositionen die bei den PP zur Verfügung stehenden formalen Mittel als Unterscheidungskriterien nicht ausreichen. Für die Disambiguierung muß auf den Kontext zurückgegriffen werden. Andererseits gibt diese Beschreibung den Anhaltspunkt für die formalen zusammenhänge von z.B. temporalen, modalen und kausalen Adverbialen.

25 - Zu der Struktur des Satzes s. oben Kap. 3.2.3 "themalose Aussagen" (S. $117 \mathrm{ff}$ ). 
26 - Vgl. dazu Rủžið̌ka (1980), 31 ff; ders.(1977); zu dem Problem der Mehrdeutigkeit nach Hebungs-Tf s. S. 168.

27 - Weitere Oberflächenstrukturen, die hier nur der Vollständigkeit wegen genannt seien, sind:

- My razgovarivali vozvrasclajas' domoj.

- My razgovarivali, kogda my vozvraščalis' domoj.

Diese Strukturen erfordern $z u$ ihrer Untersuchung einen weiteren Rahmen formaler Regeln, als er hier behandelt wird; zu den Sätzen mit Adverbialpartizip s. die neueren Arbeiten von Růžǐka (1980).

28 - Die folgenden Ausführungen beschränken sich auf die Darlegungen von Weiss zu Adverbialpartizipien. Die Diskussion um die Abgrenzung dieser Struktur von attributiv gebrauchten Partizipien kann unberücksichtigt bleiben.

29 - Weiss (1978), 258 f; vgl. dazu auch Rủžička (1980), 181 f.

30 - Für die Illustration des problems, im besonderen bei kritischer Uberprüfung der Ergebnisse des Rollentauschs bietet sich die PP (pri NP verb) an. Das hat andererseits den Nachteil, daß "pri NP verb" in mehrfacher Hinsicht ambig sein kann (s. S. $174 \mathrm{ff}$ ). So lassen sich unschwer Beispiele anführen (z.B. (115) und die Ausführungen dazu), auf die die folgende Argumentation über hierarchische verhältnisse in Sätzen mit PP temp nicht past.

31 - Der Vollständigkeit halber sei 'erwähnt, daß das Beispielset durch (4la) "Pri molitve on kurit." ergänzt werden könnte. Jedoch ist die Einbeziehung dieser Satzform in unsere Fragestellung redundant, da die Relation von (4la) zu (41) der von (43) zu (42) entspricht. 
32 - Den Hinweis auf diesen witz als - m.E. - treffende objektsprachliche Illustration meiner versuche, die hier entwickelte Konzeption für eine TS von Sätzen mit freien Adverbialen $z u$ verdeutlichen, verdanke ich $A$. de vincenz.

33 - Zu dem Begriff der "kommunikativen Bedeutung" im Rahmen von T-C-A-Analysen, vgl. Sgall, Hajičová, Benešová (1973), 47; vorher Firbas im Zusammenhang mit "kommunikativer Dynamik" (1964), $270 \mathrm{f}, \mathrm{s}$. dazu auch oben S. $115 \mathrm{f}$.

34 - Die generative TG machte auf weitere prädikative Strukturen neben dem finiten Prädikat aufmerksam, wie sie z.B. bestimmte Adjektivklassen darstellen. Unter der umfassenderen Fragestellung $z u$ prädikativen Aussagen in verschiedenen Oberflächenstrukturen sind m.w. die PP bisher anhand eines repräsentativen Korpus nicht untersucht worden.

35 - Dieser Satz - über dessen Sinn man streiten kann - macht deutlich, daß der grammatische Rollentausch $z$ wischen finitem Verb und Advb Temp ${ }_{10 c}$ an gewisse Restriktionen gebunden ist. Das hängt offensichtlich mit dem unterschiedlichen logischen Status von Prädikat und Argument zusammen (vgl. dazu Tugendhat (1976), 441). Mit diesem Erklärungszusammenhang lassen sich dann auch Nonsens-Bildungen als Folge dieses grammatischen Rollentauschs einordnen (s. Beisp. (54)).

36 - $\mathrm{zu}$ der Mehrdeutigkeit dieser Art von Sätzen in Zusammenhang mit Hebungs-Tf S. S. 149 und S. 168.

37 - Die tiefenstrukturell übergeordnete Prädikation des Adverbials kann einen niedrigeren Rang auf der Skala der kommunikativen' Dynamik erhalten (s. auch Kap. 3.2 die Ausführungen $z u$ Advb ${ }_{\text {loc }}$ in verschiedenen Satzstellungen). 
38 - Vgl. dazu detailliert Panevová (1978a).

39 - $\mathrm{zu}$ der Relation zwischen Verbalaspekt und lexikalischer Bedeutung der Verben S. Avilova (1976), z.B. $23 \mathrm{ff}$.

40 - Zu dem Begriff vgl. Mehlig (1980), 9; Avilova (1976), 23.

41 - Befragungen von native-speaker-Informanten zu dieser Art Sätze - s. auch u. S. 182 (135) - ergaben, daß die Akzeptabilität maßgeblich von Faktoren wie Vorwissen und Kontext abhängig ist, wodurch die Mehrdeutigkeit der grammatischen Struktur ihre Bestätigung findet.

42 - zu den Voraussetzungen von Aussagen über Raum und zeit vom Standpunkt der sprachanalytischen Philosophie vgl. Tugendhat (1976): "Wir kommen also nicht umhin, das Identifizierungssystem wahrnehmbarer Gegenstände - das System raumzeitlicher Relationen - als das einheitliche und umfassende System von unbegrenzt vielen Raum- und zeitstellen anzuerkennen, das allerdings auf eine endliche Mannigfaltigkeit von materiellen Gegenständen und Ereignissen als wahrnehmbare Bezugspunkte angewiesen ist. Es besteht zwischen den beiden Mannigfaltigkeiten - der der materiellen Gegenstände und der der Ereignisse einerseits und der Raum- und zeitstellen andererseits - eine wechselseitige Abhängigkeit ..." (463).

43 - Die Darlegungen beschränken sich im wesentlichen auf die Verwendung von PP in HS oder in solchen NS, die aufgrund ihrer Satzsemantik deutlich von der der PP verschieden sind. Mit besonderen formalen Restriktionen für temporale Prädikationen muB z.B. in temporalen NS gerechnet werden, wie das folgende Beispiel illustriert:

(120) Esli vvesti porjadok, kogda pri prochoそ̌denii techniCeskogo osmotra v GAI predstavljajutsja 
medicinskie spravki o sostojanii zdorov'ja voditelja, sražu že možno budet vyjavit' tech komu ne mesto za rulem. -

Die Einbettung eines $S_{\text {temp }}$ in einen temporalen $N S$ ist offensichtlich blockiert, man vgl. zu (120): *...kogda, kogda prochodjat techničeskij osmotr, predstavlajutsja medicinskie spravki ....

Die PP (pri NP verb') ist damit die obligatorische Form zum Ausdruck der konditionalen und/oder temporalen Relation zum finiten Prädikat des $\mathrm{S}_{\text {temp }}$.

44 - Vgl, weitere Beisp.:

(127) Takich predprijatij pri sodejstvii SSSR budet postroeno bolee tridcati.

(128) Pri aktivnom uCastii sovetskich èlektrotechnikov sozdavalis' ... promyslennye ob' 'ekty.

s. in diesem Zusammenhang auch die Ergebnisse zu lokalen Adverbialen und temporalen NS, S. $73 \mathrm{ff}$.

45 - Jukłin, V., Rasskazy, Moskva, 1981, S. 110.

46 - s. O. S. 46 f. Des weiteren haben Untersuchungen zu den Funktionen der Adverbialpartizipien ergeben, daB auch in dieser Hinsicht vergleichbare semantische Verhältnisse bei der Verwendung von Adverbialen und Adverbialpartizipien vorliegen. Vgl. Weiss $(1974,194)$ : "Die Wahl zwischen konditionalem, kausalem, instrumentalem oder temporalem Bezug in einem Satz wie:

(12) Zarabiając osiem patyków mogẹ utrzymać całą rodzinę." bleibt unentscheidbar. 
ANMERKUNGEN ZU KAPITEL 5 - 7:

1 - Für die Feststellung von obligatorischen Modaladverbialen werden analog $\mathrm{zu}$ den Lokaladverbialen Prädikations- und Dialog-Test angewendet. Jedoch ist im unterschied zu der quantitativ relevanten Klasse der Verben mit obligatorischem Lokaladverbial die Klasse der Verben mit obligatorischem Modaladverbial kaum von Bedeutung; dabei ist eine substitution des Adverbs durch PP nur beschränkt möglich. Vorläufig soll der Hinweis auf obligatorische Modaladverbiale als Fußnote genügen; vgl. dazu auch Bartsch (1972), 331; Steinitz (1971), 27. Beispiele für PP sind: vesti sebja po dostoinstvu; obchodit'sja s kem po dostoinstvu; ¿to-to obchoditsja bez skandala; weitere Verben mit obligatorischem Modaladverbial: vygljadet' choroso; cuvstvovat' sebja choroš; pachnut' choroso.

2 - Chomsky (1965), 136, auch Anm. 28; Lakoff, G., Linguistik und natürliche Logik, Frankf., 1971, Kap. IX, A; Bartsch (1972), $146 \mathrm{ff}, 289 \mathrm{ff}$.

3 - Dies ist m.w. bisher nicht erfolgt; s. dazu auch Diskussion zu Růziłka (1980), u. 5.1, S. 185 ff.

4 - zur Tempusvereinheitlichung in den Beispielen werden die Belege in der Regel im Präteritum angeführt.

5 - Es sei hier auf Exkurs 2 (S. $223 \mathrm{ff}$ ) verwiesen, in dem die mögliche Verneinung modaler Ausdrücke durch die Präposition "bez" zur Sprache kommt.

6 - vgl. Steinitz (1971), 57 f; Bartsch (1972), 291 zu den unterschiedlichen Voraussetzungen bei vergleichbaren oberflächenstrukturen. 
7 - $z u$ evtl. unterschiedlichen Regelabläufen bei der Zurückführung der $P P{ }_{\text {mod }}$ auf einen Satz ${ }_{\bmod }$ s. unten Kap. 5.2, S. 192 ff und Kap. 5.4, S. 208 ff.

8 - Zu den logischen Voraussetzungen für die Funktion der Verneinung von "raumzeitlichen Relationen" vgl. z.B. Tugendhat (1976) $463 \mathrm{f}$.

9 - Zu diesem Begriff vgl. Sgall, Hajið̌ova, Beneک̌ova (1973), 96 ff.

10 - $z u$ der damit angesprochenen Klasse von Modaladverbialen s.u. Kap. 5.2, bes. S. $202(64)-(65)$.

11 - vgl. im bes. loc. cit. 9.2 .1 (184 f).

12 - Bartsch (1972) $146 \mathrm{f}$; vgl. zu der Verneinung in Sätzen mit bestimmten Adverbialen auch Lakoff, G., Instrumental Adverbs and the Concept of 'Deep' Structure; in: Foundations of Language, 4, 1968, 4-29.

13 - In dieser Funktion ist die Negation in Sätzen mit Modaladverbialen der in Sätzen mit obligatorischem Lokaladverbial vergleichbar. Ich verweise hier nur auf Untersuchungen zum Deutschen, vgl. Steinitz (1971) 57 f; Bartsch (1972), 291. Es lassen sich analoge Ergebnisse für das Russische erwarten.

14 - zu Mehrdeutigkeit der Adverbialen s.o. Kap. 4.4.2, S. $172 \mathrm{ff}$.

15 - vgl. Lakoff (1968); Bartsch (1972), 290.

16 - Für dieses Beispiel mag folgender Satz deutlicher sein: Oni Živut $v$ ožidanii bol'žich izmenenij. Oni Zivut, ožidaja bol'šich izmenenij. 
17 - zu dieser Konstruktion s.u. ausführlicher Kap. 5.3 (S. $204 \mathrm{ff})$.

18 - $\mathrm{s}$. in diesem Zusammenhang auch den Hinweis auf kooperationale Adverbiale in der folgenden Anm.

19 - dagegen Beip. "žit's roskoš'ju." (Slovar' (AN) (1950 ff) Bd. 12, Sp. 1468) "s roskos' ju" ist hier kooperationales Adverbial: "Nekotorye aktrisy zili s bol'కoj roskošju: Svoi osobnjaki, lakei v livrejach ..."

20 - Das Adverbial steht, soweit lexikalisch belegt, in der Form des Adverbs, um die uberprüfung durch den Test nicht durch die Ambiguität der PP - z.B. ihren Funktionen in verschiedenen Adverbialklassen - $z u$ erschweren.

21 - Der unterschiedliche Grad der Akzeptabilität - was bei den folgenden Beispielen noch deutlich werden wird - dürfte in Zusammenhang damit stehen, daß in Folge von Wortbildungsregeln die Nominalisierungs-Tf (vgl. 45a) weit seltener möglich ist als bei den bisher analysierten Adverbialen.

22 - im Unterschied zu Sätzen mit anderen Verbklassen als Präd S. unten Kap. 5.4 ( $208 \mathrm{ff})$.

23 - zu Verben wie "perebit"" (3), "voskliknut'" (29) usw. S. unten $\operatorname{Mod}_{2}$, S. $208 \mathrm{ff}$.

24 - Auf diese Konstruktion macht auch zolotova (1973) aufmerksam. Dort werden als Belege u.a. angeführt: "V smjaten'e svacha $k$ nej bežit.", "V molłanii vernulis' $v$ stolovuju " (262). 
25 - Spraul (Schneider), H., Transformationsgrammatik und Russischunterricht - ein Versuch, Heidelberg, Forschungsbericht KADR - Arbeitspapier Nr. 5, 1973.

26 - Eine weitere Bildung mit Instrumental - inzwischen lexikalisiert - von modalen Adverbialen zeigen die Zahlwörter: vdvoem, vtroem, včetverom.

27 - Zu einem anderen Ergebnis der grammatischen Beschreibung von Ausdrücken wie "v kačestve..." kommt Nichols (1981, S. $229 \mathrm{ff})$. Bei dem in der vorliegenden Arbeit gemachten versuch steht das Prädikat von $\mathrm{S}^{2}$ zur Kennzeichnung seiner metasprachlichen Form in [] .

28 - Die Beispiele ließen sich beliebig erweitern, man vgl. noch:

"Odevalsja ja $i$ క̌l na večer $v$ velikom vozbuždenii";

"On ... v smustenii otvernulsja".; "KuXer i dva lakeja stojat pered karetoj v nedoumenii."; "Lavreckij pochodil okolo sada v smutnoj nadezde vstretit'sja s Lizoj."

29 - vgl. O. Kap. 5.2, Beisp. (39), S. 193.

30 - Dieses Beispiel eignet sich dazu, auf die mannigfaltige Mehrdeutigkeit der Formen der PP aufgrund ihrer zugehörigkeit $\mathrm{zu}$ verschiedenen Adverbialklassen aufmerksam zu machen. Man vgl. $2 u(75)$ :

(76) ... dumaet on s nevynosimoj toskoju.

(77) - Koneðno! - zakrical ja v vostorge.

(78) - Koneð̌no! - zakri ‘al ja s vostorgom.

In (77) ist die PP in dem Sinn von (75) ambig; (78) ist ein Beisp. für die Subklasse von Modaladverbialen, die u. Kap. 5.4 besprochen wird ( $\mathrm{s}$. auch S. 196 (3) und Prädikatsvariablen-Test b); für(76) dagegen scheint keiner der bis jetzt erprobten Tests $z$ u passen; intuitiv verstehen wir, daß (76) etwas Ähnliches bedeutet wie (79) Dumaet on i toskuet. - 
und würde damit żu der Klasse der kooperationalen Adverbiale gehören, die ich hier jedoch nicht untersuche (s. zu dieser Mehrdeutigkeit der PP (s NP ${ }_{\text {instr }}$ ) auch u. Kap. 5.4 (208 ff).

31 - Bei der Untersuchung der modalen Adverbiale in der Form von PP wurde deutlich, daß für die Analyse ihrer Syntax und Semantik Wortbildungsregelmäßigkeiten eine größere Rolle spielen, als das für die lokalen und temporalen Adverbiale der Fall ist. Das hängt damit zusammen, daß die NP der PP bei den Modaladverbialen mit Nomina besetzt sind, die in der Regel $\mathrm{zu}$ unterschiedlichen Klassen gehörende, abgeleitete Lexeme sind. Hier ist die Erklärung dafür $z u$ suchen, daß bei der Anwendung der Prädikatsvariablen-Tests (s. die ff Prozeduren) die Ergebnisse differieren - vgl. z.B. zu (88): kak on zanimalsja vzdorom, proischodilo važno. Nach dem Wörterbucheintrag (OŽegov) gilt als 3. Bedeutung von važnyj: gordelivo-veličestvennyj; dazu ist als Beispiel genannt: "važno (nareč.-sic!) derŽat' sebja". Die ausdrückliche Nennung der Wortklasse ist m.E. ein Zeugnis dafür, daß die Verwendung mit dieser Bedeutung relativ begrenzt ist. Die 1. Bedeutung von važnyj ist nämlich nach ožegov: imejusčij bol'šoe, osoboe znałenie, znałitel'nyj. In dieser Bedeutung ist die Verwendung des Lexems als Modaladverbial blockiert. In zusammenhang mit Problemen der Wortbildung, auf die hier nur verwiesen wird und für die eigene Untersuchungen erforderlich sind, muß auch Exkurs 1 ( $S .221$ f) gesehen werden; vergleichbare Fälle behandelt z.B. Zolotova (1976); s. dazu auch o. S. 80 f, Anm. 35 und u. S. 213 f Beispiele (104) ff.

32 - einschränkend $z u$ der satzsemantischen Analyse dieses Beispiels s. dazu u. S. 215 .

33 - vgl. Wörterbucheintrag (Oそegov): "l. napravljat' vzgljad, čto-by uvidet' kogo-čto-n." 
34 - s. Wörterbucheintrag (OŽegov): "1. obladat' sposobnostju zrenija". Ein Vergleich mit dem Deutschen ("mit Begeisterung etc. sehen") zeigt, daß es sich hier um sprachspezifische Regelmäßigkeiten handelt. Das muß im zusammenhang damit gesehen werden, daß wir mit (89), (90), vgl. auch die Ausführungen zu "vkusno", S. $215 \mathrm{f}$, über die generelle Problematik der satzsemantischen Verhältnisse in Sätzen mit Adverbialen hinaus auf die spezielleren wortsemantischen Implikationen gestoßen sind.

35 - s. dazu auch Bartsch (1972),151 f; im besonderen: "Die Adjektive in der Verwendung von Mod ${ }_{2}$ werden primär über den Handlungsvollzug durch $x_{1}, d . h$. über das Handeln von $x_{1}$ prädiziert. Es ist dann sekundär, daß diese Adjektive als Prädikate von $x_{1}$ verwendet werden können: Der Handlungsvolizug von $x_{1}$ ist $A \quad \ldots x_{1}$ handelt $A \longleftrightarrow x_{1}$ verhält sich $A \longrightarrow x_{1}$ ist $A$. Dabei ist " $A$ " ein in $\operatorname{Mod}_{2}^{1}$ verwendbares Adjektiv."

36 - vgl. die der $\mathrm{PP}_{\mathrm{Mod}_{2}}$-nahen Konstruktion: "uchodit' ot ... s trudom"; s. unten Exkurs 1, S. $221 \mathrm{f}$.

37 - Auf die damit implizierten Relationen von Syntax und Semantik machte als erster in einer zusammenhängenden Theorie Ch. J. Fillmore, The Case for Case, 1968, aufmerksam. In neueren Arbeiten zum modernen Russisch behandelt u.a. I. Zimmermann (1978) vergleichbare Probleme.

38 - Davon $\mathrm{zu}$ unterscheiden ist dagegen: gotovjat (èto) bl judo s osobennym vkusom, was auch bedeuten kann: Éto bljudo $\mathbf{s}$ osobennym vkusom, - dann liegt eine "habere"-Relation", d.h. attributive Relation vor, die in dieser Arbeit nicht behandelt wird.

39 - s. u. Exkurs 2, S. 223 ff zu PP (bez NP gen ). 
40 - Weitere Beisp. mit (s $\mathrm{NP}_{\text {gen }}$ ) vgl.:

(116) Ved' chotjat že teper' konfiskovat' knjazja, tak と̌to prichoditsja ego vozvrašcat' Cut ne s boju.

(117) Odnim slovom, spešu napropaluju! Lošadej vzjal s boju: otnjal u kakogo-to kolležskogo sovetnika ...

41 - vgl. Bartsch (1972), 154, "Die eigentlich modale Adverbial-Konstruktion liegt in der modalen Proform - 'so'".

42 - zu den Satzadverbialen vgl. Bartsch (1972), 33.

43 - Wohl aufgrund von Wortbildungsregeln, denn man vgl. die syntaktisch synonyme struktur - s. Prädikations-Test - mit Ausdrücken wie: $\ldots$ prijatno, kak .... ... udobno, kak ..., ... vredno, kak ... usw., die jedoch nicht als Modaladverbiale in der Form "s prijatnost'ju", "s vrednost'ju" auftreten; s. auch Hinweis o. S. 269, Anm. 31.

44- Vgl. oben S. $219 \mathrm{f}$ die Hinweise auf formale Zusammenhänge zwischen Modaladverbialen und Satzadverbialen.

45 - vgl. oben S. 237, Anm. 3.

46 - Unsere Untersuchungen sind auf die moderne russische Schriftsprache beschränkt. Dennoch möchten wir hier zu den Begriffen "koordinierende" und "subordinierende" sprachliche Mittel eine Beobachtung A. Issatschenkos aus seiner "Geschichte der russischen Sprache" (Bd. 1, 1980, S. 85) zitieren. Zu der im Altkirchenslavischen aus dem Griechischen entlehnten Konstruktion: Partizip + finities Verb heibt es: "Dort, wo andere Sprachen zwei beigeordnete finite Verbformen verwenden, verwandelt das Griechische eine dieser finiten Formen in ein Partizip. Hier folgt das Griechische übrigens einem im Semitischen verbreiteten Konstruktionstypus. Aus 'er antwortete und (er) sprach' wird etwas wie 'antwortend er sprach', ... aksl. otıvěštavı reče ..." 
47- Die Kongruenz können wir unberücksichtigt lassen, da sie sich auf andere sprachliche Einheiten - nämlich die Grammateme im Sinn der FGB - erstreckt als Rektion und Adjunktion, s. dazu auch Kibrik (1977) $177 \mathrm{f}$.

48 - vgl. Kibrik (1977) 178 f. 


\section{LITERATURVERZEICHNIS}

(Das Verzeichnis enthält nur die Fachliteratur, die in der vorliegenden Arbeit ausgewertet wurde).

Adamec, P. (1966), Porjadok slov v sovremennom russkom jazyke, Praha.

Adamec, P. (1974), Aktual'noe Členenie, glubinnye struktury i perifrazy. In: Papers on Functional Sentence Perspective, S. $189-195$.

Apresjan, Ju. D. (1974), LeksiCeskaja semantika. SinonimiCeskie sredstva jazyka. Moskva.

Apresjan, Ju. D. (1980), Tipy informacii dlja poverchnostnosemantiČeskogo komponenta modeli smysl $\longleftrightarrow$ tekst. Wiener Slawistischer Almanach, Sonderband 1. Wien.

Arutjunova, N. D. (1976), Predloženie i ego smysl. Moskva.

Avilova, N. S. (1976), Vid glagola i semantika glagol'nogo slova. Moskva.

Bartsch, R. (1970), Die logische Analyse von Modaladverbien. In: Linguistische Berichte, 10, S. $27-34$.

Bartsch, R. (1972), Adverbialsemantik. Frankfurt.

Bartsch, R. (1974), Logik und Grammatik. In: Zeitschrift für Germanistische Linguistik, 2, S. $206-221$.

Bartsch, R. (1975), Subcategorization of Adnominal and Adverbial Modifiers. In: Formal Semantics of Natural Language, S. $175-187$.

Bellert, J. (1969), Arguments And Predicates In The LogicoSemantic Structure of Utterances. In: Studies in Syntax and Semantics, S. $34-52$.

Bémová, A., weisheitelová, J. (1975), Model' "Smysl $\longleftrightarrow$ Tekst" $v$ sopostavlenii $s$ funkcional'noj poroždajustej model'ju. In: P Bulletin ML, 24, S. $35-50$.

Benes, E. (1973), Thema-Rhema-Gliederung und Textlinguistik. In: Studien zur Texttheorie und zur deutschen Grammatik, S. $42-62$.

Bennett, D. C. (1975), Spatial and Temporal Uses of English Prepositions. London. 
Beranová, V. (1975), $K$ valentnosti russkich glagolov dviženija. In: P Bulletin ML, 6, S. $255-262$.

Bierwisch, M. (1971), Grammatik des deutschen Verbs, Studia Grammatika II. Berlin.

Birkenmaier, w. (1977), Thema-Rhema-Gliederung und Verbalaspekt. In: Iral XV, 3, S. $209-219$.

Birkenmaier, w. (1978), Restriktive Bedingtheit im Russischen, Polnischen, Tschechischen. In: Welt der Slaven, Jg. XXIII, N.F. II, S. $248-256$.

Bolla, K., Páll, E., Papp, F. (1968), Kurs sovremennogo russkogo jazyka. Budapest.

Brekle, H. (1969), Generative Semantics vs Deep Syntax. In: Studies in Syntax and Semantics, S. $80-90$.

Brekle, H. (1970), Generative Satzsemantik und transformationelle syntax im system der englischen Nominalkomposition. München.

Brož, L. (1971), Adverbial'naja perifrastika. Praha.

Cejtlin, S. N. (1976), Sintaksičeskie modeli so značeniem psichiCeskogo sostojanija i ich sinonimika. In: Sintaksis i stilistika, S. 161 - 181 .

Chomsky, N. (1970), Aspekte der Syntax-Theorie. Frankfurt.

Chomsky, N. (1971), Deep structure, surface structure and semantic interpretation. In: Semantics, S. $183-216$.

Contributions To Functional Syntax And Semantics (1985), hrsg. v. P. Sgall. Philadelphia, Amsterdam.

Coseriu, E. (1977), Leistung und Grenzen der Transformationellen Grammatik. Tübingen.

Dahl, ర. (1969), Topic and Comment: A Study in Russian and General Transformational Grammar (Slavica Gothoburgensia). Göteborg.

Daneš, F. (1968), Typy tematických posloupností v textu (na materiále ceského textu odborného), slovo a slovesnost, 29 , S. $125-141$.

Dictionnaire de linguistique de l'Ecole de prague (1960), hrsg. v. J. Vachek. Utrecht. 
English Transformational Grammar (1968), hrsg. v. Jacobs, R., Rosenbaum, P., Waltham, Mass.

Explizite Beschreibung der Sprache und automatische Textbearbeitung. I, Terminologisches wörterbuch (1975); II, Bibliographie der linguistischen Gruppe des 2NM/MPF/KU (1975); III, Die tektogrammatische Ebene der funktionellen generativen Beschreibung (1977), Praha.

Fillmore, Ch. J. (1968), Plädoyer für Kasus. In: Kasustheorie (1971), hrsg. v. W. Abraham. Frankfurt.

Firbas, J. (1964), On Defining the Theme in Functional Sentence Analysis. In: Travaux linguistiques de Prague, I, 267 - 280. Praha.

Formal Semantics of Natural Language (1975), hrsg. v. E. Keenan. Cambridge.

Frege, G. $\left(1966^{2}\right)$, Funktion, Begriff, Bedeutung: Fünf logische Studien. Göttingen.

Freidhof, G. (1978), Kasusgrammatik und lokaler Ausdruck im Russischen. München.

Functional Generative Grammar in Prague (1973), hrsg. v. W. Klein, A. V. Stechow. Kronberg/TS.

Generative Grammar in Europe (1972), hrsg. v. F. Kiefer. Dordrecht.

Grammatika sovremennogo russkogo literaturnogo jazyka (1970), hrsg. v. N. Švedova. Moskva.

Harris, Z. (1968), Mathematical Structures of Language. New York.

Hartung, w. (1971), Die zusammengesetzten Sätze des Deutschen, Studia Grammatica IV. Berlin.

Heringer, H. J. (1968), Präpositionale Ergänzungsbestimmungen im Deutschen. In: Zeitschreift für deutsche Philologie, Bd. 87, H. 3, S. $426-457$.

Hinzer, R. (1978), N-P-N-Konstruktionen im Russischen. Versuch einer grammatisch-semantischen Analyse von $\mathrm{N}$ o(b) $\mathrm{N}^{2}$, masch.-geschr. Saarbrücken (Dipl.-Arbeit).

Issatschenko, A. (1980), Geschichte der russischen Sprache, 1. Bd. Heidelberg. 
Jakobson, R. (1935), Beitrag zur allgemeinen Kasuslehre. Gesamtbedeutungen der russischen Kasus, S. 77 - 124. Zit. nach: Form und Sinn (1974). München.

Jaksche, H. (1978), Betonung, Wortstellung und syntaktische Konstruktion im russischen Satz. In: Slavica Helvetica, 12, S. $77-85$.

Johnson, D. B. (1970), Transformations and Their Use in the Resolution of Syntactic Homomorphy. Prepositional Constructions in Contemporary Standard Russian. The Hague.

Kacnel'son, S. D. (1972), Tipologija jazyka i rę̌evoe myšlenie. Leningrad.

Kasustheorie (1971), hrsg. v. W. Abraham. Frankfurt.

Katz, J. J. (1972), Zeitliche Spezifizierungen; zustände, Vorgänge und konverse Beziehungen. In: Semantik und generative Grammatik, I, II, S. $199-260$.

Kibrik, A. E. (1977), O sootnoక̌enii ponjatija sintaksiðeskogo podXinenija s ponjatijami soglasovanija, upravlenija i primykanija. In: Problemy teoretiteskoj i éksperimental'noj lingvistiki, vyp. 8, Moskva, S. 161 179.

Koktová, E. (1977), Are Local Adverbials Derived from Temporal Sources? In: P Bulletin ML, 27, S. $45-63$.

Koschmieder, E. (1965), Beiträge zur allgemeinen Syntax. Heidelberg.

Koschmieder, E. (1975), Denken - Sprechen - Schreiben; Bayrische Akademie der Wissenschaften, Sitzungsbericht, Jg. 1975, H. 2. München.

Kovtunova, I. I. (1974), Aktual'noe Elenenie i sistema jazyka (na materiale russkogo jazyka). In: Papers on Functional Sentence Perspective, S. 142 - 151 .

Kovtunova, I. I. (1976), Sovremennyj russkij jazyk. Porjadok slov i aktual'noe לlenenie predložnija. Moskva.

Krause, J. (1975), Zur Klassifizierung der Geschehensverben. In: Akten der 1. Salzburger Frühlingstagung für Linguistik Bd. 1, S. 217 - 225 .

Krause, J. (1977), Untersuchungen über das Verb "geschehen". Tübingen. 
Krebs, D. (1974), Die Passivfunktion von -sja im russischen Satz (Versuch einer syntaktisch-semantischen Analyse), masch.- geschr. Saarbrücken (Dipl.-Arbeit).

Krylova, O. (1976), Determinanty v aspekte kommunikativnogo sintaksisa. In: Voprosy jazykoznanija, 2, S. $43-52$.

Krylova, O., Chavronina, S. (1976), Porjadok slov v russkom jazyke. Moskva.

KrŽižkova (Křlžková), E. (1967), Adverbial'naja determinacija so znaCeniem mesta $i$ napravlenija (Opyt transformacionnogo analiza). In: Voprosy jazykoznanija, 1967, 2, S. $32-48$.

KuKera, H., Trnka, K. (1975), Time in Language: Temporal Adverbial Constructions in Czech, Russian and English. Michigan.

Labov, W. (1976, 1978), Sprache im sozialen Kontext, Bd. I, 2. Königstein/Ts.

Lakoff, G. (1968), Instrumental Adverbs and the concept of Deep Structure. In: Foundation of Language, 4, S. 4- 29.

Lakoff, G. (1971), Linguistik und natürliche Logik. Frankfurt.

Machová, S. (1972), The Adverbial of Cause in a Generative Description of Czech. In: P Studies ML, 4, S. $215-228$. Abgedr. in: Functional Generative Grammar in Prague, $\mathbf{S}$. $306-317$.

Mathesius, V. (1929), zur Satzperspektive im modernen Englisch. In: Archiv für das Studium der neueren Sprachen und Literaturen, 155, S. $202-210$.

Mathesius, v. (1975), A Functional Analysis of Present Day English on a General Linguistic Basis (hrsg. v. J. Vachek). The Hague.

Mehlig, H. R. (1980), Linguistische und didaktische Uberlegungen zum Verbalaspekt im Russischen. In: Zielsprache Russisch, 1, S. 1-16.

MelCuk, I. A. (1974), Opyt teorii lingvistiðeskich modelej "Smysl" $\longleftrightarrow$ Tekst". Moskva.

Nichols, J. (1981), Predicate Nominals. A Partial Surface Syntax of Russian. Berkeley, Los Angeles, London.

OŽegov, S. J. (1972), Slovar' russkogo jazyka. Moskva. 
Padučeva, E. V. (1974), O semantike sintaksisa: Materialy k transformacionnoj grammatike russkogo jazyka. Moskva.

Panevová, J. (1967), K voprosu o grammatiCeskoj sinonimii v generativnom opisanii jazyka. In: P Studies ML, 2, S. 227 - 244, (überarbeitet (1973), On Grammatical Synonymy in Generative Description of Language. In: Functional Generative Grammar in Prague, S. 125 - 159).

Panevová, J., Sgall, P. (1972), Razrabotka sluCajnogo poroZdenija Zesskogo teksta kak proverka teoretiCeskoj ramki generativnogo opisanija Zešskogo jazyka, P Bulletin, ML, 18 , S. $29-42$.

Panevova, J., Sgall, P. (1973a), On Grammatical Synonymy in Generative Description of Language. In: Functional Generative Grammar in Prague, S. $125-159$.

Panevová, J., Sgall, P. (1973b), Verbal Aspect in an Explicit Description of Language. In: Functional Generative Grammar in Prague, S. $236-257$.

Panevová, J. (1974 - 1975), On Verbal Frames in Functional Generative Description, Part I, P Bulletin ML, 22 (1974), S. 3 - 40; Part II, P Bulletin ML, 23 (1975), S. 17 - 52 .

Panevová, J. (1977), Verbal Frames Revisited, P Bulletin ML, 28 , S. $55-71$.

Panevova, J. (1978a), Fragen der Nominalisierung zeitlicher Prädikation. In: $\mathrm{P}$ Bullet in ML, 30, S. $9-42$.

Panevova, J. (1978b), Inner Participants And Free Adverbials. In: P Studies ML, 6, S. $227-254$.

Papers on Functional Sentence Perspective (1974), hrsg. v. F. Danes. The Hague.

Pełkovskij, A. M. (1934), Russkij sintaksis $v$ nau nom osvešenii. Moskva.

Pitha, P. (1972), Nekotorye zameXanija k obrabotke morfologii obstojatel'stva mesta $v$ generativnom opisanii Cesskogo jazyka $s$ neskol'kimi urovnjami. In: P Studies ML, 3, S. $241-257$.

Pražskij lingvistǐ̌eskij kružok (1967), hrsg. v. N. A. Kondrasov. Moskva.

Problemy teoretiČeskoj i éksperimental'noj lingvistiki, vyp. 8 (1977), hrsg. v. V. A. Zvegincev. Moskva. 
Problemy teorii grammatičeskogo zaloga (1978), hrsg. v. V. Chrakovskij. Leningrad.

Procházka, O., Sgall, P. (1976), Semantic Structure of the Sentence and Formal Logic. In: P Studies ML, 5, S. $256-$ 270; (überarbeitete Fassung: Sgall, Hajičová, Procházka, 1977).

Rassudova, O. P. (1968), Upotreblenie vidov glagola v russkom jazyke. Moskva.

Reiter, N. (1975), Die Semantik deutscher und russischer Präpositionen. Wiesbaden.

Robert, C. (1964), Contribution à l'étude de quelques complements de temps en russe moderne. Paris.

Rothstein, R. A. (1974), Relevancing Marking in Polish Complements. In: Slavic Transformational Syntax, S. 53 65 .

Russkaja Grammatika (1979), Bd. 1, 2; hrsg. v. V. Barnetová et al. Praha.

Russkaja Grammatika (1982), Bd. 1, 2; hrsg. v. N. Švedova et al. Moskva.

Rủzið̌ka, R. (1966), Studien zur Theorie der russischen Syntax. Berlin.

Rủzicka, R. (1970a), Zur Struktur des Adverbials im modernen Russisch. In: Jazyk i Čelovek. Publikacii otdelenija strukturnoj i prikladnoj lingvistiki, vyp. 4 (Festschrift für P. S. Kuznecov), Moskva, S. $239-261$.

Rủžiłka, R. (1970b), Semantik des Verbs und Syntax des Satzes. In: Zeitschrift für Slawistik, 5, S. $627-644$.

Rüžǐka, R. (1977), Syntaktische und semantische Aspekte der Hebungs-Regel im modernen Russischen. In: Zeitschrift für Slawistik, 2, S. $3-7$.

Rủziłka, R. (1980), Studien zum Verhältnis von Syntax und Semantik im modernen Russischen, I. Berlin.

Schmid, w. (1970), Skizze einer allgemeinen Theorie der Wortarten. In: (Mainz) Akademie der Wissenschaften und der Literatur. Abhandlungen der geistes- und sozialwissenschaftlichen Klasse, Nr. 5, Jg. 1970.

Schüller, M. (1976), N-P-N-Konstruktionen im Russischen. Versuch einer grammatisch-semantischen Analyse von NP po $\mathrm{N}^{2}$, masch.-geschr. Saarbrücken (Dipl.-Arbeit). 
Schwarze, Ch. (1976), Pseudologik und Natürliche Generative Grammatik - Anmerkungen zur "Adverbialsemantik" von Renate Bartsch. In: Papiere zur Linguistik, 11, S. 56 - 109.

Semantics (1971), hrsg. v. D. D. Steinberg, L. A. Jacobovits. London, New York.

Semantik und generative Grammatik, I, II (1972), hrsg. v. F. Kiefer.

Sgall, P. (1964), Zu Fragen der Ebenen im Sprachsystem. In: Travaux linguistiques de Prague, 1, S. 95 - 106.

Sgall, P. (1967), Zur Eingliederung der Semantik in die Sprachbeschreibung. In: Folia linguistica, I (1/2), S. 18 - 22.

Sgall, P. (1967), Functional Sentence Perspective in a Generative Description. In: P Studies ML, 2, S. $203-225$.

Sgall, P., Nebeský, L., Goralčiková, A., Hajičova, E. (1969), A Functional Approach to Syntax. New York.

Sgall, P., Hajiłová, E., Benesová, E. (1973), Topic, Focus and Generative Semantics, Kronberg/Ts.

Sgall, P., Hajicová, E. (1973), A Functional Generative Description (Background and Framework). In: Functional Generative Grammar in Prague, S. 1- 52.

Sgall, P. (1974), Zur Stellung der Thema-Rhema-Gliederung in der Sprachbeschreibung. In: Papers on Functional Sentence Perspective, S. $54-74$.

Sgall, P. (1975), Stellung und Aufgaben der algebraischen Linguistik (Einführungsstudie), II. In: $\mathrm{P}$ Bulletin ML, 23, S. $53-74$.

Sgall, P. (1976), Zum Stand der Thema-Rhema-Forschung in der Tschechoslowakei. In: Osteuropa, S. $163-182$.

Sgall, P. (1977), K metodam opisanija sintaksisa. In: P Bulletin ML, 27, S. $5-20$.

Sgall, P., Hajičová, E., Procházka, O. (1977), Linguistic Semantics and Intensional Structure. In: philologica pragensia, 20, S. $179-194$.

Sgall, P., Hajił̌ová, E., Panevová, J. (1986), The Meaning of the Sentence in Its Semantic and Pragmatic Aspects. Prague. 
Siegrist, L. (1976), Bibliographie zu Studien über das deutsche und englische Adverbial. Tübingen.

Sintaksis i stilistika (1976), hrsg. v. G. A. Zolotova. Moskva.

Skalička, V. (1935), Asimmetrið̌nyj dualizm jazykovych edinic, abgedruckt in: Pražskij lingvistið̌eskij kružok, S. 119 127.

Slavic Transformational Syntax (1976), hrsg. v. R. Brecht, C. Chvany. Michigan.

Slovar' sovremennogo russkogo literaturnogo jazyka, AN SSSR. Moskva, (1950 ff).

Spraul (Schneider), H. (1973), Transformationsgrammatik und Russischunterricht - ein Versuch. Heidelberg. Forschungsbericht KADR-Arbeitspapier, Nr. 5, 23 S.

Spraul (Schneider), H. (1978), Zum Problem der präpositionalen Ergänzung des Verbs im Satz - am Beispiel des Russischen. In: Russisch als Fremdsprache, Hamburger Beiträge für Russischlehrer, Bd. 17, S. 119 - 137.

Sachmatov, A.A. (1925), Sintaksis russkogo jazyka, Leningrad, Nachdruck. The Hague, 1963.

Šmelev, D.N. (1976), SintaksiČeskaja Xlenimost' vyskazyvanija $v$ sovremennom russkom jazyke. Moskva.

Svedova, N. Ju. (1964), Determinirujustij ob''ekt i determinirujuscee obstojatel'stvo kak samostojatel'nye rasprostraniteli predlozenija. In: Voprosy jazykoznanija, 6, S. $77-93$.

Steinitz, R. (1971), Adverbialsyntax. Berlin.

Steube, A. (1969), Uber die Beziehung der Zeitadverbien zum Satz und ihre Selektionsbeziehungen $z u$ den relevanten Elementen im Satz. In: ASG-Bericht, Nr. 3. Berlin.

Streit, G. (1976), Zur wiedergabe der deutschen Präposition "mit" in der russischen Schriftsprache der Gegenwart, masch.-geschr., Göttingen (Staatsexamensarbeit).

Studien zur Texttheorie und zur deutschen Grammatik (1973), hrsg. v. H. Sitta/K. Brinker. Düsseldorf.

Studies in syntax and Semantics (1969), hrsg. v. F. Kiefer. Dordrecht. 
Thümmel, W. (1977), Argumente gegen die parafrasenmethode. In: Linguistische Berichte, 51, S. $21-51$.

Trenczek, H. (1976), N-P-N-Konstruktionen im Russischen. Versuch eiper grammatisch-semantischen Analyse von $\mathrm{N}^{I}$ za $\mathrm{N}^{2}$ und $\mathrm{N}^{\mathrm{S}} \mathrm{N}^{2}$, masch.-geschr., Saarbrücken (Dipl.Arbeit).

Trends in Soviet Theoretical Linguistics (1973), hrsg. v. F. Kiefer. Dordrecht.

Tugendhat, E. (1976), Vorlesungen zur Einführung in die sprachanalytische Philosophie. Frankfurt.

Ulířová, L. (1976), Optional Constituents in Thema-Rhema Structure. In: P Studies ML, 5, S. $309-320$.

Ungeheuer, G. (1969), Paraphrase und syntaktische Tiefenstruktur. In: Folia Linguistica, 3, S. $178-227$.

Vinogradov, V. V. (1972), Russkij jazyk. Moskva.

Vsevolodova, M., Paršukova, Z., Potapova, G., Cukanova, A. (1973), Sposoby vyraženija obstojatel'stvennych otnosenij (prostranstvo, vrema, prið̌ina). Moskva.

Weiss, D. (1974), Zur Modellierung polnischer Gerundialkonstruktionen. In: Slavistische Linguistik, S. $187-209$.

Weiss, D. (1976), Semantische und pragmatische Aspekte kausal-konjunktionaler Satzgefüge am Beispiel des Russischen. In: Slavistische Linguistik, S. $221-260$.

Weiss, D. (1978), Nichtrestriktive Attribution vs sekundäre Prädikation: Zwei Typen von "Backgrounding". In: Slavica Helvetica, 12 , S. $251-271$.

Wierzbizka, A. (1972), In Search of A Semantic Model of Time And Space. In: Generative Grammar in Europe, S. $616-628$.

Wunderlich, D. (1970), Tempus und zeitreferenz im Deutschen. München.

Zimmermann, I. (1972), Die substantivische Satzform. In: Zeitschrift für Slawistik, 17, S. $674-684$.

Zimmermann, I. (1978), SintaksiČeskie funkcii aktantov, zalog i perechodnost'. In: problemy teorii grammatičeskogo zaloga, S. $71-79$.

Zolotova, G. A. (1973), Oł̌erki funkcional'nogo sintaksisa russkogo jazyka. Moskva. 
Zolotova, G. A. (1976), O sintaksiðeskich svojstvach imen kałestva. In: Sintaksis i stilistika, S. $130-160$.

Zolotova, G. A. (1982), Kommunikativnye aspekty russkogo sintaksisa. Moskva. 
Summary

This study examines adverbials from a syntactic point of view: that is, from the standpoint of their relationships to the sentence or to individual sentence constituents. This question forms the focus of the study, since it remains open on the basis of the existing research results in standard grammars (Russkaja Grammatika, 1982) as well as in more recent descriptions of syntax.

The main problems connected herewith exist in categorial and subcategorial areas. For instance, do the adverbials form a lexical base category, which serves as the head of a main category, or are they a derivative "small-clause" variant of the standard form sentence? The problems also touch upon conditions of movement, scope, structural analyzability in context, etc.

In particular, the unsettled questions deal with the cases in which the adverbials are not dominated by the verb kernel. The dependence on the VP has been demonstrated for the subclass of the obligatory adverbials (Steinitz, 1971). The present study likewise excludes adverbial clauses; it is confined to selected classes (location, time and manner) of the free adverbials in Russian, which appear in the form of prepositional phrases (PP Advb).

It is difficult to deal with the questions raised in a methodically explicit manner, since the PP Advb in Russian, in contrast to other main clause constituents, form a formally weak determined class of syntactic expressions. While the syntactic relationships of the other main clause constituents are determined by congruence and government, this is not true for PP ${ }_{A d v b}$. Furthermore, the combinatorial possibilities of sentences with PP Advb appear not to be limited, semantic selectional restrictions are not displayed, and the position 
of the $\mathrm{PP}_{\mathrm{Advb}}$ in the word order of the sentence appears to be free. However, distributional analysis draws attention to restrictions on the occurrence of the adverbials, from which it can be concluded that there are rules for their use.

These regularities are partially systematic and can be applied as well to more than one language; they are partially dependent on the context, not only in the broader sense, which we mean when we speak of language in a social context, but also in the narrower sense of the given text. This study is restricted to the examination of the systematic factors, but the contextual factors are also brought up, especially in cases for which the mutual contingency of system-dependent and context-dependent factors is relevant.

According to the outlined conditions of the object of this study and under consideration of the current state of research, the hypothesis is put forward that the PP is a "small-clause" variant of the standard form of sentence. In order to describe the syntax of sentences with PP Advb' it is necessary to draw up a sentence connection model. As a theoretical frame I use a new variant of generative transformational grammar, the "Functional Generative Grammar" (FGG) (Sgall et alii, 1973, revised 1986). This enables us to formulate the free adverbials as deep-structural predications. Questions connected with conditions of movement in the word order of the sentence and beyond the limits of the sentence or with the scope of predication reflect regularities of the sentence perspective which belong to the linguistic system in the FGG concept.

The subclassification of adverbials is modelled on examinations of predicate logic. The syntactic problem is linked to the problem of sentence semantics by the question posed herewith about the argument(s) of deep structure predication. 
The verification of the thus conceived syntactic madel of sentences with PP ${ }_{A d v b}$ is performed by means of various kinds of tests, which were designed under consideration of the requirement of syntacic synonymy in the sense of FGG.

The three tests should be mentioned in particular: the predication test, the negation test and the theme-rheme test. With the help of the predication test, rules can be formulated for the various syntactic relationships of the individual subclasses of adverbials to the other sentence constituents. Adverbials of location, in contrast to adverbials of time and manner, relate to "things" and "events"; adverbials of time and manner, on the other hand, relate only to "events". The negation test reveals truth conditions of the statement, through which the adverbials of manner are differentiated from the adverbials of location and time. The theme-rheme segmentation test reveals rules for the conditions of movement of the $\mathrm{PP}_{\mathrm{Adub}}$ and the scope of the predication: in particular, borderline cases of rheme and theme play an interesting role here.

As a result of the problem outlined at the beginning, it can be determined that the occurrence of $\mathrm{PP}{ }_{\mathrm{Advb}}$ in the sentence shows certain regularities of the Advb linguistic system. Furthermore, it becomes clear that the regulating mechanisms which comprise on the one hand sentence-semantic factors, and on the other hand the sentence perspective, are arranged hierarchically. The structuring of the statement through topic and comment limits the predication possibilities of the adverbials. For the surface sentence, this means that the syntactic function of the $\mathrm{PP}_{\mathrm{Advb}}$ is limited by the sentence perspective.

These conclusions thus form a contribution to a theory of the syntax of the simple sentence. 
В книге исследуются адвербиальные детермннанты /далее "адвербиал"/ с синтаксической точки зрения, точнее с точки зрения их связей с предложением или с отдельными членами предложения. Этот вопрос стоит в центре внимания исследования, потому что по имеюиимся научным результатам, отраженным как в академических трудах /Русская Грамматика, Москва, 1982г./ так и в специальных синтаксических концепциях новеишего времени его надо считать открытым. Связанные $\mathrm{C}$ ним основные проблемы затрагивают категориальные и субкатегориальные аспекты классификации языка, например: являются ли адвербиалы лексической базовои категориеи или же они являются трансФормированной синтагмои /"small-clause"-варнантом/ предложения? Далее, проблемы касаются порядка слов, возможного изменения позиций членов предложения и связанного с этимдиапозона соотнесенности адвербиалов с другими компонентами предложения, а также их контекстуального анализа.

Выяснение требуют в первую очередь те случаи функционирования адвербиалов, когда нельзя утверждать, что они находятся в прямой зависимости от ядра-глагола. Такая зависимость свойтвенна подтипу облигаторных адвербиалов/Steinitz, 1971/. Данная работа не затрагивает также вводно-оценочных слов /"Satzadverbiale"/. Она ограничивается изучением тех факультативных адвербиалов, которые пр́едставлены локальным, темпоральным и модальным классами, и выступают в формах предложных конструкций /PP Advb $_{\text {. }}$

Поставленные вопросы трудно подвергать методически эксплицитной обработке по той причине, что PP Advb являются в отличие от других основных членов предложения в русском языкє формально слабо детерминируемым классом синтаксических единиц. В то время как основным членам предложения свойственнс 
установление сннтакснческих связей на основе согласования и управлення, - у адвербналов отсутствуют подобные формальные показатели. Помимо того, представляется, что сочетаемость предложения с PP ${ }_{\text {Advb }}$ не ограничена, что семантические критерия для сочетаемости вряд ли можно обнаружить н что позиция адвербиала в цепочке предложения свободно варьируется.

Однако дистрибутивныи анализ вызывает сомнения относнтельно правильности таких выводов, поскольку они выявляют ограничения в употребленин адвербиалов, и это дает право предпо-

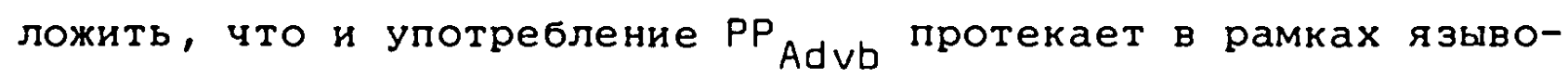
вых закономерностей.

Отчасти это снстемные закономерности, которые, кроме того, считаются общеязыковыми, отчасти же они обусловлены контекстом. Контекст понимается здесь и в широком значении, как "социальныя контекст", и в узком - как конкретный текст. данная работа ограничивается изучением обусловленных системоя факторов, она затрагивает однако н контекстуальные факторы, в особенности если речв идет о таких случаях, когда взаимообусловленность и тех и других является релевантной.

С учетом вкратце изложенных данных о самом объекте анализа н С учетом научных концепций его изучения на данном этапе в настоящеи работе сформулирована гипотеза, что PP Advb являются трансформированной синтагмой /"small clause вариантом/ предложения. Следовательно, для представления синтаксиса предложения с PP ${ }_{A d v b}$ требуется модель, описывающая пути соединения предложении. В качестве общих теоретических рамок используется новый вариант генеративной трансформационной грамматики, а нменно "Functional Senerative Grammar (FGG) /Sgall et alii, Prague, 1973, переработанной в $1986 \mathrm{r} . /$. 
В такой концепции адвербиалы можно определить как предикации глубинной структуры. Вопросы, затрагивающие варианты порядка слов и изменения позиций $P_{A d v b}$ в пределах одного предложения и в контексте, а также вопросы, касающиеся диапозона предикации, - отражают закономерности актуального членения предложения, которое в концепции FGG отнесено к системе языка.

Субкласснфикация адвербиалов проводится с опорой на работы нз области "философии языка", в которых высказывания трактуются как преднкацин разного типа. Тут возникает вопрос о типе аргумента той или иной предикация, и тем самым проблема синтаксических свойств предложения связывается с проблемой его семантикн.

Проверка предложенной модели синтаксиса обеспечивается рядоя тестов, при формулировке которых были учтены /следуя FGC/ принципы для определения синтаксической синонимия высказывания. Используются, в частности, тест предикация, тест негация, и тест актуального членения.

С помощью теста предикации можно раскрыть закономерности, определяюине различия в синтакснческих связях отдельных подклассов адвербиалов с другими членами предложения. Так, например, в отличие от адвербналов темпоральных и образа действия, локальные адвербиалы "относятся" как к "предметам", так и к "событиям" . Зато адвербиалы темпоральные н образа действия "относятся" только к "событиям". Тест негация раскрывает условия нстинности высказывания, обуслосливающие различия между адвербиалами образа действня, С одной стороны, н локальными и темпоральными, - с другой.

Тест актуального членения выявляет закономерности в различных позициях адвербиалов и их диапозона преднкации. Особый интерес вызывают позиции "на границе" между темой и ремой.

Подытоживая результаты нсследования, можно сказать, что упо

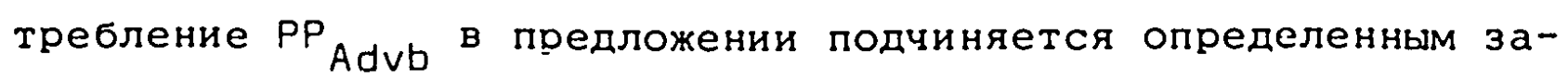


кономерностям языковой системы. Более того, становится ясньм, что множество правил, определяюших семантику предложения, и множество правил актуального членения соотносятся друг с другом в определенной очередности: членение предложения на тему и рему обусловливает диапозон предикации. Из этого следует, что на уровне поверхностнои структуры синтаксическая функция PP Advb ограничивается актуальным членением.

Можно сделать вывод, что эти результаты вносят и новые аспекты в теорию синтаксиса простого предложения. 